\title{
Twisted Kloosterman sums and cubic exponential sums
}

\author{
Dissertation \\ zur Erlangung des Doktorgrades \\ der Mathematisch-Naturwissenschaftlichen Fakultäten \\ der Georg-August-Universität zu Göttingen
}

vorgelegt von

Benoît Louvel

aus Rennes

Göttingen 2008 
D7

Referent: S.J. Patterson

Korreferent: P. Michel

Tag der mündlichen Prüfung: 15.12.2008 


\section{Contents}

Introduction $\quad 5$

$1 \quad$ Asymptotic behaviour of $K_{2}(m, n, c) \quad 11$

$1.1 \quad$ Automorphic forms on the upper half-plane . . . . . . . . . . . . . . . 13

1.1 .1 Maaß forms and modular forms . . . . . . . . . . . . . . . . . 14

1.1 .2 Poincaré series . . . . . . . . . . . . . . . . . . . 17

1.2 The Goldfeld-Sarnak method $\ldots \ldots \ldots$. . . . . . . . . . . . . 20

1.2 .1 The Kloosterman-Selberg zeta function . . . . . . . . . . . . . . 20

1.2 .2 Asymptotic behaviour . . . . . . . . . . . . . . 22

1.3 Theta functions of half-integral weight $\ldots \ldots \ldots \ldots \ldots \ldots . \ldots \ldots$

1.3.1 A result of Serre and Stark . . . . . . . . . . . . . . . . 26

1.3 .2 Orthonormalisation of theta functions . . . . . . . . . . . . 29

1.4 Determination of the constant . . . . . . . . . . . . . . . . . . 34

1.4.1 Choice of the cusps and of the level . . . . . . . . . . . . . . 34

\begin{tabular}{|ll|l}
\hline 1.4 .2 & Proof of Theorem $\mid 1.1 \| \ldots \ldots \ldots \ldots$ & $\ldots \ldots \ldots \ldots$ \\
\hline
\end{tabular}

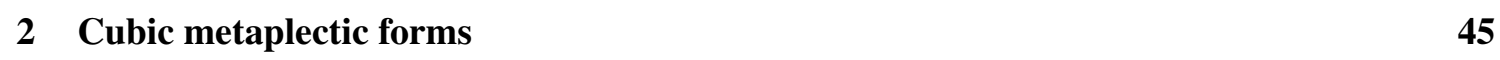

$2.1 \quad$ Metaplectic forms on $\left.\mathrm{SL}_{2}(\mathbb{Z}[\omega])\right] \ldots \ldots \ldots \ldots \ldots$

$2.1 .1 \quad K$-types and metaplectic forms $\ldots \ldots \ldots \ldots$. . . . . . . . . . 49

2.1 .2 Eisenstein series, theta functions and the spectral decomposition theorem 52

2.1 .3 Poincaré series . . . . . . . . . . . . . . . . . . . 54

2.2 Summation formulae of Kuznetsov type $\ldots \ldots \ldots \ldots \ldots$

$2.2 .1 \quad$ Lebedev transform and the spectral sum formula . . . . . . . . . . . 56

2.2 .2 On the spectrum of the metaplectic group . . . . . . . . . . . 61

2.2 .3 Bessel transform and the Kloosterman sum formula . . . . . . . . . . 66

2.3 Metaplectic group and metaplectic representations . . . . . . . . . . . . 71

2.3 .1 Kubota symbol and metaplectic group . . . . . . . . . . . . . . 71

2.3 .2 Metaplectic and automorphic representations . . . . . . . . . . 74

2.4 Shimura correspondence . . . . . . . . . . . . . . . . . . . 77

$2.4 .1 \quad$ Definitions and results $\ldots \ldots \ldots \ldots 77$

2.4 .2 Classical interpretation $\ldots \ldots \ldots \ldots \ldots$ 
$\begin{array}{lll}3 & \text { Asymptotic behaviour of } K_{3}(m, n, c) & \mathbf{8 1}\end{array}$

3.1 The Kubota-Patterson theta function _ . . . . . . . . . . . . . . . . . . . 83

3.1.1 The Maaß-Selberg relations . . . . . . . . . . . . . . . . . 83

$3.1 .2 \quad$ Properties of the cubic theta functions $\ldots \ldots \ldots$. . . . . . . 88

3.2 Relations between theta functions $\ldots \ldots \ldots$. . . . . . . . . . . . 91

$3.2 .1 \quad$ Auxiliary results $\ldots \ldots \ldots \ldots 9 . \ldots \ldots$

3.2 .2 Scalar product of theta functions $\ldots \ldots \ldots 9$

3.3 Some conclusions . . . . . . . . . . . . . . . . 111

4 Cancellation of $S\left(a X^{3}+b X, c\right)$, for $c$ allmost prime 115

4.1 A non-trivial estimate in average . . . . . . . . . . . . . . . . . . 117

4.2 Sieve argument on $\mathbb{Z}[\omega]] \ldots \ldots \ldots \ldots$

$4.2 .1 \quad$ The Selberg sieve . . . . . . . . . . . . . . . . . . . . . . . . . 122

4.2 .2 Precise estimates . . . . . . . . . . . . . . . . . 130

$4.3 \quad$ An upper bound for $S\left(a X^{3}+b X, c\right) \ldots \ldots \ldots \ldots \ldots \ldots$

$4.3 .1 \quad$ An upper bound for $\mathcal{A}^{ \pm} \ldots \ldots \ldots \ldots$. . . . . . . . . . . . . . . . . . . . . . . . . . . . . . . . . . . .

$4.3 .2 \quad$ An upper bound for $\mathcal{B} \ldots \ldots \ldots \ldots \ldots$

$4.4 \quad$ A lower bound for $S\left(a X^{3}+b X, c\right) \ldots \ldots \ldots \ldots \ldots \ldots$

4.4 .1 Sato-Tate vertical law . . . . . . . . . . . . . . . . . . 145

4.4 .2 Absolute lower bound . . . . . . . . . . . . . . . . . . . . . . . 146

\begin{tabular}{|ll}
\hline Acknowledgements & 153
\end{tabular}

\begin{tabular}{ll}
\hline Lebenslauf & 155
\end{tabular}

\begin{tabular}{ll}
\hline Abstract & 157
\end{tabular} 


\section{Introduction}

This dissertation is concerned with exponential sums of the form

$$
S_{\chi}(f, c)=\sum_{x(c)} \chi(x) e\left(\frac{f(x)}{c}\right),
$$

for $c$ in the ring of integers $R$ of a number field $k, f$ a rational function, $\chi$ a character modulo $c$ and $e(z)=\exp \left(2 i \pi \operatorname{Tr}_{k / \mathbb{Q}}(z)\right)$. They are finite sums, where $x$ runs through the finite set of representative of $R / c R$ and where we agree to write $x(c)$ instead of $x(\bmod c)$. Historically, a first motivation for the study of these sums arises from the diophantine analysis, where many problems are reduced to the evaluation of such sums. For example, Hilbert's Eleventh Problem asks about the representability of integers in a number field by an integral quadratic form. For the special case of diagonal forms, the 'circle method' was introduced by Hardy and Littlewood to study asymptotically the number of solutions of

$$
F(x)=x_{1}^{2}+x_{2}^{2}+\ldots+x_{s}^{2}=m, \quad \text { for } s \geqslant 5 .
$$

In 1926, Kloosterman ([21]) studied this problem over $\mathbb{Q}$ for $s=4$, and was led to introduce the so-called Kloosterman sums

$$
K(m, n, c)=\sum_{\substack{x, x^{*}(c) \\ x x^{*} \equiv 1(c)}} \exp \left(\frac{2 i \pi\left(m x+n x^{-1}\right)}{c}\right),
$$

defined for $m, n, c \in \mathbb{Z}$. He succeeded in obtaining the non trivial individual estimate

$$
|K(m, n, p)| \leqslant E p^{3 / 4},
$$

which allowed him to solve the problem for diagonal forms in four variables. Besides the significance of individual bound as (3) for Kloosterman sums, it is expected that bound on average are equally important. Around 1960, working on some additive problems, Linnik introduced a 'dispersion method' in which Kloosterman sums play a fundamental role, and he conjectured ([28]) a cancellation among these sums. At the same time, in his seminal work on Fourier coefficients of modular forms ([40]), Selberg studied the zeta function associated to Kloosterman sums and formulated the same conjecture, which asserts that

$$
\sum_{c<X} \frac{K(m, n, c)}{\sqrt{c}} \ll X^{1 / 2+\varepsilon}, \quad \forall \varepsilon>0 .
$$

The major breakthrough in this problem came from Kuznetsov in 1979 ([26]), who proved it for any $\varepsilon>1 / 6$. These is the kind of problems we shall be interested in, for some special sums $S_{\chi}(f, c)$. 
A further motivation comes from the cohomological interpretation of the Sato-Tate conjecture about elliptic curves without complex multiplication. For an elliptic curve $E$ over $\mathbb{Q}$, if $a_{p}(E)$ is defined by $a_{p}(E)=\sharp E\left(\mathbb{F}_{p}\right)-1-p$, where $\sharp E\left(\mathbb{F}_{p}\right)$ is the number of points on $E$ over $\mathbb{F}_{p}$, then the Hasse upper bound asserts that

$$
\left|a_{p}(E)\right| \leqslant 2 \sqrt{p} .
$$

According to (5), we define a family of angles $\theta_{p}(E)$ by

$$
\frac{a_{p}(E)}{2 \sqrt{p}}=\cos \theta_{p}(E),
$$

and the question is, if these angles are uniformly distributed for some measure. In the late 1940s, the Weil conjectures about the Hasse-Weil zeta function attached to a variety gave the key to the uniform distribution of the angles $\theta_{p}(E)$ when $E$ has complex multiplication, since a result of Deuring asserts that in this case, the Hasse-Weil zeta function is expressed in terms of Hecke $L$-function about which we know enough analytic results. Around 1960, Sato and Tate arrived independently to the conjecture that such a uniform distribution measure also exists for elliptic curves without multiplication, and is given by

$$
\mu_{S-T}=\frac{2}{\pi} \int \sin ^{2} \theta \mathrm{d} \theta \quad \text { on }[0, \pi] .
$$

By a cohomological approach, Serre (see [41], I-25, I-26) proved that one can deduce distribution results from knowledge about the analytic continuation of the $L$-function attached to $E$. Coming back to Kloosterman sums, the proof by Weil from the Riemann hypothesis for curves led him to improve the individual bound (3) for Kloosterman sum to

$$
|K(m, n, p)| \leqslant E p^{1 / 2},
$$

which is the best possible. Within the cohomological framework, N. Katz (see [18], conj. 1.2.5) formulated the uniform distribution of the angles of Kloosterman sums according to the Sato-Tate law, a 'folklore conjecture' which arose in the 1970s. More precisely, if

$$
\frac{K(1, a, p)}{2 \sqrt{p}}=\cos \theta_{p, a} \quad\left(0 \leqslant \theta_{p, a} \leqslant \pi\right)
$$

then it was conjectured that for any $0 \leqslant \alpha<\beta \leqslant \pi$,

$$
\frac{\left|\left\{p: X \leqslant p<2 X, \alpha \leqslant \theta_{p, 1} \leqslant \beta\right\}\right|}{|\{p: X \leqslant p<2 X\}|} \longrightarrow \frac{2}{\pi} \int_{\alpha}^{\beta} \sin ^{2} \theta \mathrm{d} \theta, \quad \text { as } X \rightarrow+\infty .
$$

Notice that in the angles that we consider, both the parameter $p$ and the other parameter (either $E$ or a) can vary. We shall distinguish these cases by speaking from the 'horizontal' case and from the 'vertical' one, respectively. As well as the numerous numerical verifications supporting the horizontal conjecture, one other reason to believe in the Sato-Tate conjecture for elliptic curves or for Kloosterman sums came from Birch ([1]) and Katz ([19] Ex. 13.6), who proved the vertical case of this conjecture, respectively for elliptic curves and for Kloosterman sums. Finally, the Sato-Tate conjecture for curves was proved in 2006 by Clozel, Harris, Shepherd-Barron \& Taylor under the condition that $j(E)$ is not an integer.

We come now to our object of interest. Actually in [1], after proving the vertical asymptotic behaviour for elliptic curves, Birch conjectured the same 'vertical' result for the cubic sums 
$S\left(a X^{3}+b X, c\right)$; this conjecture was latter proved by Livné ([29] and [30]). The same dichotomy as the one between elliptic curves with or without complex multiplication occurs for cubic sums, and, as a matter of fact, a uniform distribution over the primes in the case where $b=0$, i.e. for the sums $S\left(a X^{3}, p\right)$, was proved by Heath-Brown and Patterson ([15]). This parallel with Hecke's work leads naturally to conjecture the horizontal Sato-Tate law for cubic sums with $b \neq 0$. For both cases, i.e. for Kloosterman sums or for cubic sums, even though the 'vertical' question was solved, the 'horizontal' one remains still open. We shall be here mostly interested in cubic sums, and we shall present some progress towards the cancellation and the uniform distribution of such sums over the Eisenstein integers, i.e. the integers of the Eisenstein ring $\mathbb{Z}[\omega]$, where $\omega=\exp (2 i \pi / 3)$. Another object of interest will be the twisted Kloosterman sums. These are analogues of the classical Kloosterman sums when, in the definition (1), we choose the character $\chi$ to be the Legendre-Jacobi symbol of order $j$. By this we mean that these sums, that we shall denote by $K_{j}$, are given by $S_{(\dot{\bar{c}})_{j}}\left(\frac{X^{2}+1}{X}, c\right)$, i.e.

$$
K_{j}(m, n, c)=\sum_{\substack{x, x^{*}(c) \\ x x^{*} \equiv 1(c)}}\left(\frac{x}{c}\right)_{j} e\left(\left(\frac{m x+n x^{*}}{c}\right)\right) .
$$

We shall study the cases where $j=2$ or $j=3$, and speak about quadratic and cubic Kloosterman sums, respectively. We remark that the Kloosterman sums defined in (2) correspond to the case where $j=1$, and therefore, we shall call $K_{1}(m, n, c)$ the classical Kloosterman sum. The reason for studying the case where $j=3$ is that there exists a close relation between the cubic exponential sum $S\left(a X^{3}+b X, c\right)$ and the cubic Kloosterman sum $K_{3}(m, n, c)$. The study of the case where $j=2$ follows the same general steps as for the cubic case, although the techniques used are easier to deal with.

Let us present the method that we follow: The problem of asymptotic behaviour of Kloosterman sums was undertaken by Kuznetsov, along the lines described by Selberg in [40]. This is based on the theory of automorphic forms. We shall parallel this, and combine spectral properties of automorphic forms with properties of the zeta function attached to the sums $K_{j}(m, n, c)$ to obtain a summation formula for the sums $K_{j}(m, n, c)$. We shall then naturally be confronted to the minimal eigenvalue problem which brings into play theta functions. For $j=2$ these theta functions are the classical ones, namely the twists by Dirichlet characters of the function

$$
\theta(z)=\sum_{n \in \mathbb{Z}} \exp \left(i \pi n^{2} z\right)
$$

For $j=3$, we shall work with the cubic analogues of $\theta(z)$, which are metaplectic forms constructed as residues of Eisenstein series; these functions are described in [32]. Our work aims at improving the results of Livné and Patterson (Theorem 1.1 of [31]) about the asymptotic behaviour of the cubic Kloosterman sums; we shall also give the quadratic analogue result. As for the uniform distribution problems over primes, it is believed that one acquires a better understanding by working first with integers and then by applying a sieve argument. Actually, the uniform distribution of $K_{2}(m, n, c)$ is already proved (in [17] over the integers, and in [5] over the primes), but such results are not known for $S\left(a X^{3}+b X, c\right)$; more surprisingly, even the problem of distribution of the signs of the Kloosterman sums $K(m, n, c)$ remains open. Notice that a result of uniform distribution implies the asymptotic distribution in absolute value; for example, the horizontal Sato-Tate conjecture for Kloosterman sums implies by partial summation 


$$
\sum_{p \leqslant X}\left|\frac{K(1,1, p)}{2 \sqrt{p}}\right| \sim \frac{4}{3 \pi} \frac{X}{\log X} .
$$

Indeed, upper bound and lower bound of this kind, i.e. with absolute value, are possible to derive ([10]), but the small improvements gained compared with the trivial estimation show that the cancellation expected among Kloosterman sums is more due to the change of sign than to the smallness of the norm. A way was found by Fouvry and Michel ([11]) to prove that this change of signs occurs for $K(m, n, c)$, at least for $c$ being almost prime, i.e. when the number of primes of $c$ is bounded. Our goal is to adapt their method to the cubic exponential sums, and one of the main result is to show that the sum of cubic exponential sums can actually be considered as a rest term. We prove this fact by using the theory of metaplectic forms, but, as in [15], one could expect a bias toward the $S\left(a X^{3}+b X, c\right)$ having a main term due to the existence of an exceptional eigenvalue of the Laplacian. This exceptional term comes from the cubic theta functions, but can be controlled in average over the level, leading to some non-trivial estimate. More precisely, it is expected that the classical Kloosterman sums satisfy

$$
\{p \leqslant X: K(m, n, p)>0\}=\frac{1}{2} \pi(X)+\mathcal{O}\left(\frac{X^{1 / 2}}{\log X}\right),
$$

the same being true for the Kloosterman sums of negative sign, and one could conjecture that the cubic exponential sums satisfy

$$
\left\{p \leqslant X: S\left(a X^{3}+b X, p\right)>0\right\}=\frac{1}{2} \pi(X)+C \frac{X^{5 / 6}}{\log X}+\mathcal{O}\left(\frac{X^{1 / 2}}{\log X}\right) .
$$

This kind of behaviour was already speculated in [31] p. 108-109.

We now describe the content of the thesis in more detail. In Chapter 1 we study the asymptotic distribution of $K_{2}(m, n, c)$. The summation formula over $\mathbb{Q}$ is presented in its simple form, i.e. without making explicit the contribution of the whole spectrum; In this way, we obtain quickly a formula for the asymptotic constant. In Chapter 2 we give all necessary results about automorphic forms as well as a more complete summation formula over $Q(\omega)$, where all the spectrum of the Laplacian occurs. In Chapter 3, we study the asymptotic constant of $K_{3}(m, n, c)$, i.e. we look at the basis problem for cubic theta functions. This should lead us, in a future work, to the determination of the constant $C$ appearing in (12). In Chapter 4 we develop some sieve argument to obtain an upper bound for $S\left(a X^{3}+b X, c\right)$; a fundamental role is played by the the complete summation formula of Chapter 2. Nevertheless, as for the Kloosterman sums $K(m, n, c)$, the remainder term is of the order of $\frac{X}{\log X \log \log X}$, and hides the contribution of the theta term. We then use the vertical Sato-Tate law for $S\left(a X^{3}+b X, c\right)$ to obtain a lower bound, and conclude to the change of signs when $c$ is almost prime. 


\section{Notations}

Unless otherwise stated, we make the following convention:

- The inverse of $x$ modulo a given integer will be denoted by $x^{*}$. Therefore, we shall write the sum

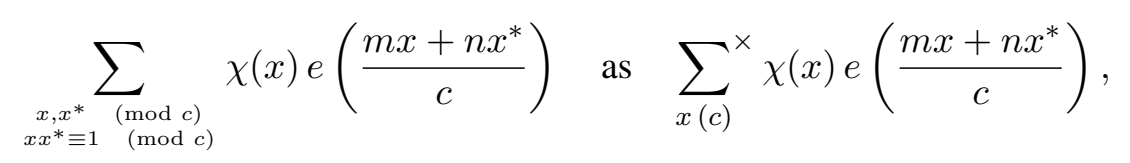

where the star means the restriction to the representatives $x$ coprime with $c$.

- For any complex number $z$, the complex conjugate will be written $\bar{z}$.

- For any matrix $g=\left(\begin{array}{ll}\alpha & \beta \\ \gamma & \delta\end{array}\right)$, we define $a(g)=\alpha, b(g)=\beta, c(g)=\gamma$ and $d(g)=\delta$.

- The symbol $\delta$ will be the Kronecker delta symbol, i.e.

$$
\delta_{a, b}= \begin{cases}1 & \text { if } a=b \\ 0 & \text { if } a \neq b\end{cases}
$$

and similarly,

$$
\delta_{P}= \begin{cases}1 & \text { if the assertion } P \text { is true } \\ 0 & \text { otherwise }\end{cases}
$$

- We shall denote the sign of a real number $x$ by $\operatorname{sgn}(x)$. 



\section{Asymptotic behaviour of $K_{2}(m, n, c)$}

The quadratic Kloosterman sums $K_{2}(m, n, c)$ appear in the work of Salié (see [38], (54) p. 102), where the following relation is proved:

$$
K_{2}(m, m, p)=\left(\frac{m}{p}\right)_{2} \varepsilon_{p} p \sum_{x^{2} \equiv 4 m^{2}(p)} \exp \left(\frac{2 i \pi x}{p}\right),
$$

where $\varepsilon_{d}=1$ if $d \equiv 1(\bmod 4)$ and $\varepsilon_{d}=i$ id $d \equiv 3(\bmod 4)$. This formula can be generalized, see for example (38) p. 438 of [5].

In this chapter, we study the asymptotic behaviour of the sum $K_{2}(m, n, c)$. We shall use the spectral theory of Maaß forms to obtain the asymptotic formula, and the theta functions to construct an explicit basis of the space of automorphic forms for which the eigenvalue of the Laplace operator is minimal. Our main goal in this chapter will be to prove the following theorem.

Theorem 1.1. Let $f$ be an odd positive integer and let $\chi$ be a primitive Dirichlet character of conductor $f$. Let $D$ be an odd positive integer. Let $m, n \in \mathbb{Z}$. Then for any $\varepsilon>0$, we have

$$
\sum_{\substack{c \equiv 0(D), 2 \\ c \leqslant X}} K_{c}(m, n, c) \overline{\varepsilon_{c}} \chi\left(\frac{c}{D}\right)=C(D, \chi, m, n) X^{3 / 2}+\mathcal{O}\left(X^{5 / 4+\varepsilon}\right) .
$$

with

For $C=0$, if $f$ is not square-free, or if the square-free parts of $m$ and $n$ are not equal and divisible by $f$.

If these conditions are met, then $m$ and $n$ have to be of the form

$$
m=t f s^{2} m^{\prime 2} \quad n=t f s^{2} n^{\prime 2}
$$

for some

- square-free $t$ coprime with $f$ such that $t^{3} \mid D$,

- some s such that $s^{2} \mid \frac{D}{t^{3}}$ and $p|s \Rightarrow p| t$,

- some $m^{\prime}, n^{\prime}$ coprime with $t$.

Then, if

$$
\frac{D}{t^{3}}=X_{0} s^{2} X^{2}, \text { with } X_{0} \text { square-free and } X \text { coprime with } t
$$

and if

$$
U^{\prime}=\left\{\prod p: p \mid X, \operatorname{ord}_{p}\left(\operatorname{gcd}\left(X, m^{\prime}, n^{\prime}\right)\right)<\operatorname{ord}_{p}(X)\right\},
$$

then 


$$
C(D, \chi, m, n)=\frac{32}{3 \pi^{2}} \frac{t^{3 / 2} s}{\varphi(t) D} \prod_{p \mid D f} \frac{p}{p+1} \chi_{t}(f) \chi_{t}\left(m^{\prime} n^{\prime}\right) \operatorname{gcd}\left(X, m^{\prime}, n^{\prime}\right) \frac{U^{\prime}}{\varphi\left(U^{\prime}\right)}
$$

In Section 1.1 and in Section 1.2, we shall work in the general context of a discrete subgroup $G$ of $\mathrm{SL}_{2}(\mathbb{Z})$, a real weight $k$ and a multiplicative system associated to $k$. In Section 1.1 the theory of automorphic forms will be developed, and in Section 1.2 we shall present an argument introduced by Goldfeld and Sarnak, which will enable us to derive the asymptotic formula in a simpler way as the one developed by Kuznetsov in [26]. Namely, such an asymptotic formula as the one from Theorem 1.1, is classically deduced by methods from analytic number theory once one has enough analytic properties of the associated zeta function. The main contribution of Goldfeld and Sarnak is, precisely, to obtain the polynomial growth of the Kloosterman-Selberg zeta function in the critical vertical strip. In Section 1.3 , we shall restrict ourselves to the congruence subgroups $\Lambda$ and $\Gamma_{0}(N)$ of $\mathrm{SL}_{2}(\mathbb{Z})$, fix the weight $k=1 / 2$, and study the theta functions, following the work of Serre and Stark [42]. Finally, we will see in Section 1.4 how the results of Section 1.3 will allow us to come back to our arithmetical problem, i.e. to prove the formula on the asymptotic constant of Theorem 1.1 . 


\subsection{Automorphic forms on the upper half-plane}

In this section, we shall first recall the definitions and properties of modular forms and Maaß forms, and then study the Poincaré series. Let us define the angle of a complex number as a real number in $[0,2 \pi[$. In particular, for any $\alpha \in \mathbb{R}$, we have

$$
z^{\alpha}=|z|^{\alpha} e^{i \alpha \arg (z)}, \quad \forall z \in \mathbb{C}, \arg (z) \in[0,2 \pi[.
$$

We shall use the notation $g^{\prime}(z)=(c z+d)^{-2}$, where $g=\left(\begin{array}{ll}a & b \\ c & d\end{array}\right) \in \mathrm{SL}_{2}(\mathbb{R})$.

Definition 1.1. Let $k$ be a real number. The factor system of weight $k$ is the application

$$
\begin{gathered}
\sigma_{k}: \mathrm{SL}_{2}(\mathbb{R}) \times \mathrm{SL}_{2}(\mathbb{R}) \longrightarrow \mathbb{C}^{*}, \\
(g, h) \mapsto(g h)^{\prime}(z)^{k / 2} g^{\prime}(h(z))^{-k / 2} h^{\prime}(z)^{-k / 2} .
\end{gathered}
$$

The following property holds (See [35], §2):

$$
\sigma_{k}(a, b) \sigma_{k}(a b, c)=\sigma_{k}(a, b c) \sigma_{k}(b, c) .
$$

Definition 1.2. Let $k \in \mathbb{R}$ and let $G$ be a discrete subgroup of $\mathrm{SL}_{2}(\mathbb{R})$. A multiplicative system for $G$ of weight $k$ is an application $\psi: G \longrightarrow \mathbb{C}$ satisfying

$$
\psi\left(g_{1} g_{2}\right)=\psi\left(g_{1}\right) \psi\left(g_{2}\right) \sigma_{k}\left(g_{1}, g_{2}\right), \quad \forall g_{1}, g_{2} \in G .
$$

Remark 1.1. Let $g, h \in \mathrm{SL}_{2}(\mathbb{Z})$. If a function $f$ defined on $\mathbb{H}$ satisfies

$$
\begin{aligned}
g^{\prime}(z)^{k / 2} f(g(z)) & =\psi(g) f(z) \\
h^{\prime}(z)^{k / 2} f(h(z)) & =\psi(h) f(z)
\end{aligned}
$$

for two constants $C_{g}$ and $C_{h}$, then one deduces that $f$ satisfies

$$
s^{\prime}(z)^{k / 2} f(s(z))=\psi(s) f(z), \quad \forall s \in\langle g, h\rangle,
$$

where $\langle g, h\rangle$ is the group generated by $g$ and $h$, and that the application $s \mapsto \psi(s)$ is a multiplicative system for the group $\langle g, h\rangle$ of weight $k$.

Let us define

$$
j_{g}(z)=\frac{g^{\prime}(z)}{\left|g^{\prime}(z)\right|}
$$

Then, considering the equality $\left|(g h)^{\prime}(z)^{k}\right|=\left|g^{\prime}(h(z))^{k}\right|\left|h^{\prime}(z)^{k}\right|$, one shows that

$$
j_{g h}(z)^{k / 2}=j_{g}(h(z))^{k / 2} j_{h}(z)^{k / 2} \sigma_{k}(g, h) .
$$

It it possible to determine $\sigma_{k}$ explicitly. Let us consider $g=\left(\begin{array}{ll}a & b \\ c & d\end{array}\right), h=\left(\begin{array}{ll}a^{\prime} & b^{\prime} \\ c^{\prime} & d^{\prime}\end{array}\right)$ and $g h=$ $\left(\begin{array}{ll}a^{\prime \prime} & b^{\prime \prime} \\ c^{\prime \prime} & d^{\prime \prime}\end{array}\right)$. Then $\sigma_{k}(g, h)$ is defined by

$$
\left(\left(c^{\prime \prime} z+d^{\prime \prime}\right)^{-2}\right)^{k / 2}=\left((\operatorname{ch}(z)+d)^{-2}\right)^{k / 2}\left(\left(c^{\prime} z+d^{\prime}\right)^{-2}\right)^{k / 2} \sigma_{k}(g, h) .
$$

Defining for any complex number $z$ the factor 


$$
\omega(z)= \begin{cases}e^{i k \pi} & \text { if } 0<\arg (z) \leqslant \pi \\ e^{2 i k \pi} & \text { if } \pi<\arg (z) \leqslant 2 \pi \\ 1 & \text { if } \arg (z)=0\end{cases}
$$

one can shows that

$$
\left(z^{-2}\right)^{k / 2}=z^{-k} \omega(z)
$$

We obtain

$$
e^{-i k \arg \left(c^{\prime \prime} z+d^{\prime \prime}\right)} \omega\left(c^{\prime \prime} z+d^{\prime \prime}\right)=e^{-i k \arg (\operatorname{ch}(z)+d)} \omega(\operatorname{ch}(z)+d) e^{-i k \arg \left(c^{\prime} z+d^{\prime}\right)} \omega\left(c^{\prime} z+d^{\prime}\right) \sigma_{k}(g, h) .
$$

One sees that $\arg \left(c^{\prime \prime} z+d^{\prime \prime}\right)-\arg (c h(z)+d)-\arg \left(c^{\prime} z+d^{\prime}\right)$ is 0 when $\left(c, c^{\prime}>0\right)$ or when $\left(c c^{\prime}<0\right.$ and $c^{\prime \prime}<0$ ), and it is $-2 \pi$ otherwise. The cases $c=0, c^{\prime}=0$ and $c^{\prime \prime}=0$ have to be considered separately. Define $x(\gamma)$ by

$$
x(\gamma)=\left\{\begin{array}{ll}
c, & \text { if } c \neq 0, \\
-d, & \text { if } c=0,
\end{array}, \text { for } \gamma=\left(\begin{array}{ll}
a & b \\
c & d
\end{array}\right) .\right.
$$

Then we obtain the following table:

\begin{tabular}{|c|c|c|c|}
\hline $\operatorname{sign}(\mathrm{x}(\mathrm{g}))$ & $\operatorname{sign}(\mathrm{x}(\mathrm{h}))$ & $\operatorname{sign}(\mathrm{x}(\mathrm{gh}))$ & $\sigma(g, h)$ \\
\hline+ & + & + & $e^{-i k \pi}$ \\
+ & + & - & 1 \\
+ & - & + & 1 \\
+ & - & - & $e^{-i k \pi}$ \\
- & + & + & 1 \\
- & + & - & $e^{-i k \pi}$ \\
- & - & + & $e^{-i k \pi}$ \\
- & - & - & 1 \\
\hline
\end{tabular}

\subsubsection{Maaß forms and modular forms}

The Poincaré upper half plane is $\mathbb{H}=\mathbb{R} \times \mathbb{R}_{+}^{*}$. If $g=\left(\begin{array}{ll}a & b \\ c & d\end{array}\right) \in \mathrm{GL}_{2}^{+}(\mathbb{R})$, the action of $g$ on $\mathbb{H}$ is given by

$$
g(z)=\frac{a z+b}{c z+d}
$$

A point $s \in \mathbb{R} \cup\{\infty\}$ is a cusp of $G$ if it is fixed by some parabolic element of $G$. Then $G$ acts on $\mathbb{H} \cup\{$ cusps of $G\}$. We say that $z_{1}$ and $z_{2}$ are $G$-equivalent if there is some element $g$ of $G$ such that $z_{1}=g\left(z_{2}\right)$. As $\{\infty\}$ is $\mathrm{SL}_{2}(\mathbb{Z})$-equivalent to $\mathbb{Q}$, any cusp $s$ of $G$ can be written as $s=\sigma^{-1}(\infty)$ for some $\sigma^{-1} \in \mathrm{SL}_{2}(\mathbb{Z})$. When working with cusps, we will assume that we work with equivalent classes, i.e. if $\sigma^{-1}(\infty)$ and $\tau^{-1}(\infty)$ are two given cusps, which are $G$-equivalent, then we take $\sigma=\tau$.

We make the assumption that $-I d \in G$ and that $\psi(-I d)=1$. Let us now define the width of a cusp $\sigma^{-1}(\infty)$ of $G$, with $\sigma \in \mathrm{SL}_{2}(\mathbb{Z})$, as the smallest positive integer $q_{\sigma}$ such that $\sigma^{-1} T^{q_{\sigma}} \sigma \in G_{\sigma}$, where $G_{\sigma}$ is the stabiliser of $\sigma^{-1}(\infty)$. Define also $\varkappa_{\sigma} \in[0,1[$ by 


$$
\psi\left(\sigma^{-1}\left(\begin{array}{cc}
1 & q_{\sigma} \\
0 & 1
\end{array}\right) \sigma\right)=e\left(-\varkappa_{\sigma}\right) .
$$

With these notations, for any $g_{\sigma} \in G_{\sigma}$ and for any multiplicative system $\psi$, one has $\psi\left(g_{\sigma}\right)=$ $\psi\left(-g_{\sigma}\right)$. We also have

$$
\psi\left(g_{\sigma}\right)=e\left(-n \varkappa_{\sigma}\right) \bar{\sigma}_{k}\left(\sigma, g_{\sigma}\right), \quad \text { if } \quad g_{\sigma}=\sigma^{-1}\left(\begin{array}{cc}
1 & n q_{\sigma} \\
0 & 1
\end{array}\right) \sigma .
$$

Definition 1.3 (modular forms). Let $G$ be a discrete subgroup of $\mathrm{SL}_{2}(\mathbb{R})$ and $\psi$ a multiplicative system for $G$ of weight $k$. A modular form is a function $f: \mathbb{H} \longrightarrow \mathbb{C}$, holomorphic on $\mathbb{H}$ and at the cusps of $G$ which satisfies

$$
g^{\prime}(z)^{k / 2} f(g(z))=\psi(g) f(z) \quad \forall z \in \mathbb{H}, \forall g \in G .
$$

The space of modular forms is denoted by $\operatorname{Mod}(G, k, \psi)$.

Here, the condition that $f$ is holomorphic at the cusps of $G$ means that there exists some $\alpha \geqslant 0$ such that for any $\sigma \in \mathrm{SL}_{2}(\mathbb{R})$,

$$
\left(\sigma^{-1}\right)^{\prime}(z)^{k / 2} f\left(\sigma^{-1}(z)\right)=O\left(\Im(z)^{\alpha}\right), \quad \text { as } z \rightarrow \infty .
$$

If $\alpha=0$, then $f$ is said to be a cusp form. The Fourier expansion of $f$ is given by

$$
\left(\sigma^{-1}\right)^{\prime}(z)^{k / 2} f\left(\sigma^{-1}(z)\right)=\sum_{n \in q_{\sigma}^{-1} \mathbb{Z}} a_{f}(\sigma, n) e\left(\left(n-\frac{\varkappa_{\sigma}}{q_{\sigma}}\right) z\right) .
$$

It can be shown that the condition that $f$ is holomorphic to the cusps of $G$ is equivalent with $a_{f}(\sigma, n)=0$ for $n-\varkappa_{\sigma}<0$ and that the condition that $f$ is a cusp form is equivalent with $a_{f}(\sigma, n)=0$ for $n-\varkappa_{\sigma} \leqslant 0$, for every cusp $\sigma^{-1}(\infty)$.

For any real number $k$, the Laplacian is defined by

$$
\Delta=y^{2}\left(\frac{\partial^{2}}{\partial x^{2}}+\frac{\partial^{2}}{\partial y^{2}}\right)-i k y \frac{\partial}{\partial x} .
$$

Definition 1.4 (Maaß forms). A Maaß form is a function $f: \mathbb{H} \longrightarrow \mathbb{C}$ on $\mathbb{H}$ of polynomial growth at each cusp of $G$, eigenvalue of the Laplacian, and which satisfies

$$
j_{g}(z)^{k / 2} f(g(z))=\psi(g) f(z) \quad \forall z \in \mathbb{H}, g \in G .
$$

The space of Maaß forms is denoted by $\operatorname{Maa}(G, \psi, k)$.

Writing $z=x+i y$, one sees that a Maaß form $f \in \operatorname{Maa}(G, k, \psi)$ has a Fourier expansion

$$
j_{\sigma^{-1}}(z)^{k / 2} f\left(\sigma^{-1}(z)\right)=\sum_{n \in q_{\sigma}^{-1} \mathbb{Z}} F(\sigma, n)(y) e\left(\left(n-\frac{\varkappa_{\sigma}}{q_{\sigma}}\right) x\right),
$$

where

$$
F(\sigma, n)(y)=\frac{1}{q_{\sigma}} \int_{0}^{q_{\sigma}} j_{\sigma^{-1}}(z)^{k / 2} f\left(\sigma^{-1}(z)\right) e\left(-\left(n-\frac{\varkappa_{\sigma}}{q_{\sigma}}\right) x\right) d x
$$


This is shown in [37] §2. In the case of a Maaß form $f$, we know that $F(n, \sigma)$ is a multiple of a Bessel function. More precisely if the eigenvalue $\lambda$ satisfies $\lambda=s(1-s)$, where $s$ is called the spectral parameter, there exists (see [37] p.301) coefficients $\rho_{f}(\sigma, n) \in \mathbb{C}$ such that,

$$
F(\sigma, n)(y)= \begin{cases}\rho_{f}(\sigma, n) W_{\frac{k}{2} \operatorname{sgn}\left(n-\frac{\varkappa_{\sigma}}{q_{\sigma}}\right), s-1 / 2}\left(4 \pi\left|n-\frac{\varkappa_{\sigma}}{q_{\sigma}}\right| y\right) & \text { if } n \neq 0, \\ \rho_{f}(\sigma, 0)^{\prime} y^{s}+\rho_{f}(\sigma, 0) y^{1-s} & \text { if } n=0 .\end{cases}
$$

For modular forms, a scalar product is defined by

$$
\left\langle f_{1}, f_{2}\right\rangle=\int_{G \backslash \mathbb{H}} f_{1}(z) \overline{f_{2}(z)} y^{k} \frac{\mathrm{d} x \mathrm{~d} y}{y^{2}} .
$$

Similarly, for Maaß forms, a scalar product is defined by

$$
\left\langle f_{1}, f_{2}\right\rangle=\int_{G \backslash \mathbb{H}} f_{1}(z) \overline{f_{2}(z)} \frac{\mathrm{d} x \mathrm{~d} y}{y^{2}} .
$$

The subspace of modular forms which are square integrable is $\mathrm{L}_{\text {Mod }}^{2}(G, \psi, k)$ and the subspace of Maaß forms which are square integrable is $\mathrm{L}_{\text {Maaß }}^{2}(G, \psi, k)$. It is the sum over the eigenvalues $\lambda$ of the subspaces $\mathrm{L}_{\lambda}^{2}(G, \psi, k)$ of forms such that $(\Delta+\lambda) f=0$. Moreover, if $f \in \mathrm{L}_{\text {Maaß }}^{2}(G, k, \psi)$, then $\rho_{f}(\sigma, 0)^{\prime}=0$, in the Fourier expansion (1.1.7). It is conjectured that eigenvalues $\lambda=s(1-$ $s) \leqslant \frac{1}{4}$ do not occur, i.e. that the spectral parameters $s$ lie all on the vertical line $\frac{1}{2}+i t$. For a given weight $k$, what one knows is the following lower bound

$$
\lambda \geqslant \frac{k}{2}\left(1-\frac{k}{2}\right)
$$

The bound (1.1.8) is derived from the results of Roelcke (see Satz 5.4 of [37], or [39] Prop. 1.2).

Proposition 1.1. Let $k=\frac{1}{2}$. Let $G$ be given and let $\psi$ be a multiplicative system of weight $\frac{1}{2}$ relative to $G$. Then there is a bijection

$$
\begin{aligned}
\mathrm{L}_{\text {Mod }}^{2}\left(G \backslash \mathbb{H}, \psi, \frac{1}{2}\right) & \cong \mathrm{L}_{\frac{3}{16}}^{2}\left(G \backslash \mathbb{H}, \psi, \frac{1}{2}\right), \\
f(z) & \mapsto y^{\frac{1}{4}} f(z) .
\end{aligned}
$$

If the Fourier expansion of $f$ is given as in (1.1.5) and if the Fourier expansion of $y^{\frac{1}{4}} f$ is given as in 1.1.7), then

$$
\rho_{f}(\sigma, n)=a_{f}(\sigma, n)\left(4 \pi\left|n-\frac{\varkappa_{\sigma}}{q_{\sigma}}\right|\right)^{-1 / 4}, \quad \forall n \neq 0 .
$$

Proof. Every modular form $f$ of weight $k$ gives a Maaß form $g(z):=f(z) y^{k / 2}$ of weight $k$ and of eigenvalue $\frac{k}{2}\left(1-\frac{k}{2}\right)$, and this holds a fortiori over the square integrable forms. In the opposite direction, the condition for a Maaß form $g$ of minimal eigenvalue to be sent on a modular form $f$ through $f(z):=g(z) y^{-k / 2}$ is that the constant term of $g$ should be of the shape $\rho(0, \sigma) y^{k / 2}$. Combined with the condition for $g$ to be square integrable, we see that a Maaß form $g$ of eigenvalue $s(1-s)$ with $\Re(s) \geqslant 1 / 2$ gives rise to a modular form $f(z):=g(z) y^{-k / 2}$ if and only if $1-s=$ $k / 2$, i.e. $k<1$. In particular, for $k=\frac{1}{2}$, there is a bijection 


$$
\begin{aligned}
\mathrm{L}_{\text {Mod }}^{2}\left(G \backslash \mathbb{H}, \psi, \frac{1}{2}\right) & \cong \mathrm{L}_{\frac{3}{16}}^{2}\left(G \backslash \mathbb{H}, \psi, \frac{1}{2}\right), \\
f(z) & \mapsto y^{\frac{1}{4}} f(z) .
\end{aligned}
$$

Suppose that $f$ is given by

$$
f\left(\sigma^{-1}(z)\right)=\left(\sigma^{-1}\right)^{\prime}(z)^{-1 / 4}\left(a_{f}(\sigma, 0)+\sum a_{f}(\sigma, n) e(n z)\right)
$$

then

$$
f\left(\sigma^{-1}(z)\right) \Im\left(\sigma^{-1}(z)\right)^{1 / 4}=j_{\sigma^{-1}}(z)^{1 / 4}\left(a_{f}(\sigma, 0) y^{1 / 4}+\sum a_{f}(\sigma, n) y^{1 / 4} e(n z)\right) .
$$

On the other side, from the formula

$$
|y|^{-\frac{1}{4}} W_{\frac{1}{4} \operatorname{sgn}(y), \frac{1}{4}}(|y|)=e^{-y / 2},
$$

we obtain, for any Maaß form $g$,

$$
\begin{aligned}
g\left(\sigma^{-1}(z)\right) & =j_{\sigma^{-1}}(z)^{-1 / 4}\left(\rho_{g}(\sigma, 0) y^{\frac{1}{4}}+\sum_{0 \neq n \in \mathbb{Z}} \rho_{g}(\sigma, n) W_{\frac{1}{4} \operatorname{sgn}(n),-\frac{1}{4}}(4 \pi|n| y) e(n x)\right) \\
& =j_{\sigma^{-1}}(z)^{-1 / 4}\left(\rho_{g}(\sigma, 0) y^{\frac{1}{4}}+\sum_{0 \neq n \in \mathbb{Z}} \rho_{g}(\sigma, n)|4 \pi n y|^{1 / 4} e^{-2 \pi n y} e(n x)\right) \\
& =j_{\sigma^{-1}}(z)^{-1 / 4}\left(\rho_{g}(\sigma, 0) y^{\frac{1}{4}}+(4 \pi y)^{1 / 4} \sum_{0 \neq n \in \mathbb{Z}} \rho_{g}(\sigma, n)|n|^{1 / 4} e(n z)\right) .
\end{aligned}
$$

Since the two expressions in 1.1 .9 ) and (1.1.11) are equal, we arrive to the relation

$$
a_{f}(\sigma, n)=\rho_{f}(\sigma, n)(4 \pi|n|)^{1 / 4}, \quad \forall n \neq 0
$$

\subsubsection{Poincaré series}

As analogues of the non holomorphic Poincaré series known since Petersson, we present here the Poincare series as they were introduced by Selberg in [40]. Let $\sigma^{-1}(\infty)$ be a cusp of $G$, $\sigma^{-1} \in \mathrm{SL}_{2}(\mathbb{Z})$. For $m \in q_{\sigma}^{-1} \mathbb{Z}-\{0\}$ one defines

$$
f_{m, \sigma}(z, s)=y^{s} e\left(\left(m-\frac{\varkappa_{\sigma}}{q_{\sigma}}\right) x\right) \exp \left(-2 \pi\left|m-\frac{\varkappa_{\sigma}}{q_{\sigma}}\right| y\right) .
$$

The Poincaré series associated to $m$ and to the cusp $\sigma^{-1}(\infty)$ is given by

$$
P_{m, \sigma}(z, s)=\sum_{g \in G_{\sigma} \backslash G} \overline{\psi(g) \sigma_{k}(\sigma, g)} j_{\sigma g}(z)^{k / 2} f_{m, \sigma}(\sigma g(z), s), \quad z \in \mathbb{H}, s \in \mathbb{C}
$$


One verifies that these series are well defined using (1.1.4). As a function of $s, P_{m, \sigma}(z, s)$ is holomorphic in $\Re(s)>1$ and as a function of $z, P_{m, \sigma}(z, s)$ satisfies

$$
j_{g}(z)^{k / 2} f(g(z))=\psi(g) f(z) \quad \forall z \in \mathbb{H}, \forall g \in G .
$$

Moreover it lies in $L^{2}$, but it is not an eigenfunction of $\Delta$. Actually, it satisfies

$$
[\Delta+s(1-s)] P_{m, \sigma}(z, s)=-4 \pi m\left(s-\frac{k}{2}\right) P_{m, \sigma}(z, s+1) .
$$

As the discrete spectrum of the Laplacian intersects $[1 / 2,1]$ in a finite set, $R_{s(1-s)}$ is holomorphic in $\Re(s) \geqslant 1 / 2$ with at most a finite number of poles in $[1 / 2,1]$. This shows the analytic continuation of $P_{m, \sigma}(z, s)$ to $\Re(s)>\frac{1}{2}$, with a finite number of poles, which are the spectral parameters of $\Delta$. Inherited from those of $P_{m, \sigma}(z, s), \operatorname{Res}_{s=s_{i}} P_{m, \sigma}(z, s)$ posses the properties of transformation according to $G$ and to be square integrable. Moreover, $\operatorname{Res}_{s=s_{i}} P_{m, \sigma}(z, s)$ is an eigenfunction of the Laplacian for the spectral parameter $s_{i}$. Thus $\operatorname{Res}_{s=s_{i}} P_{m, \sigma}(z, s) \in \mathrm{L}_{\lambda_{i}}^{2}(G \backslash \mathbb{H}, \psi, k)$, which means that if $\{u\}$ forms an orthonormal basis of it, then

$$
\operatorname{Res}_{s=s_{i}} P_{m, \sigma}(z, s)=\sum_{u}\left\langle\operatorname{Res}_{s=s_{i}} P_{m, \sigma}(z, s), u(z)\right\rangle u(z)=\sum_{u} \operatorname{Res}_{s=s_{i}}\left\langle P_{m, \sigma}(z, s), u(z)\right\rangle u(z) .
$$

Proposition 1.2. Let $f \in \mathrm{L}_{\lambda}^{2}(G \backslash \mathbb{H}, \psi, k)$, with $\lambda=s_{f}\left(1-s_{f}\right)$. Let $\sigma^{-1}(\infty)$ be an essential cusp of $G$. Let the Fourier expansion of $f$ be given by

$$
\begin{aligned}
f\left(\sigma^{-1}(z)\right)= & j_{\sigma^{-1}}(z)^{-k / 2}\left(\rho_{f}(\sigma, 0) y^{1-s_{f}}+\right. \\
& \left.\sum_{0 \neq n \in \Lambda_{\sigma}^{\wedge}} \rho_{f}(\sigma, n) W_{\frac{k}{2} \operatorname{sgn}\left(n-\frac{\varkappa_{\sigma}}{q_{\sigma}}\right), \frac{1}{2}-s_{f}}\left(4 \pi\left|n-\frac{\varkappa_{\sigma}}{q_{\sigma}}\right| y\right) e\left(\left(n-\frac{\varkappa_{\sigma}}{q_{\sigma}}\right) x\right)\right) .
\end{aligned}
$$

Then

$$
\left\langle P_{m, \sigma}(\cdot, s), f\right\rangle=q_{\sigma} e^{-i k \pi} \overline{\rho_{f}(\sigma, m)}\left(4 \pi\left|m-\frac{\varkappa_{\sigma}}{q_{\sigma}}\right|\right)^{1-s} \frac{\Gamma\left(s-\overline{s_{f}}\right) \Gamma\left(s+\overline{s_{f}}-1\right)}{\Gamma\left(s-\frac{k}{2} \operatorname{sgn}\left(m-\frac{\varkappa_{\sigma}}{q_{\sigma}}\right)\right)}
$$

and if $\mathcal{B}_{i}$ denotes an orthonormal basis of $\mathrm{L}_{\lambda_{i}}^{2}(G \backslash \mathbb{H}, \psi, k)$, with $\lambda_{i}=s_{i}\left(1-s_{i}\right)$ for a spectral parameter $s_{i}$, then

$\operatorname{Res}_{s=s_{i}} P_{m, \sigma}(z, s)=q_{\sigma} e^{-i k \pi}\left(4 \pi\left|m-\frac{\varkappa_{\sigma}}{q_{\sigma}}\right|\right)^{1-s_{i}} \frac{\Gamma\left(2 s_{i}-1\right)}{\Gamma\left(s_{i}-\frac{k}{2} \operatorname{sgn}\left(m-\frac{\varkappa_{\sigma}}{q_{\sigma}}\right)\right)} \sum_{u \in \mathcal{B}_{i}} \frac{\rho_{u}(\sigma, m)}{\rho_{i}} u(z)$.

where $\rho_{u}(\sigma, \cdot)$ is the Fourier coefficient of $u$ at $\sigma^{-1}(\infty)$.

It is known that twisted Kloosterman sums arise as Fourier coefficients of Poincaré series. To show this, we need to define a geometric analogue of the Kloosterman sums $K_{2}(m, n, c)$.

Definition 1.5. Let $\sigma^{-1}(\infty)$ and $\tau^{-1}(\infty)$ be two essential cusps of $G$. Let $m \in \Lambda_{\sigma}^{\wedge}-\{0\}$ and $n \in \Lambda_{\tau}^{\wedge}-\{0\}$. Then, for any $c \in \mathbb{Z}$, we define 


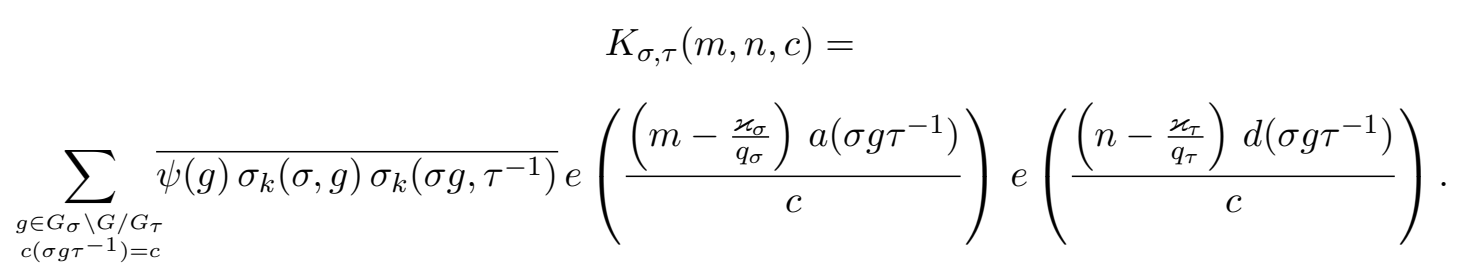

Remark 1.2. The sum $K_{\sigma, \tau}(m, n, c)$ will be the geometrical analogue of the sums $K_{2}(m, n, c)$, once we have chosen a suitable multiplicative system $\psi$ and a convenient group $G$. We shall use the same notation in Chapter 2 and in Chapter 3 for the analogue of the sum $K_{3}(m, n, c)$, but the context should make clear to which we refer.

Proposition 1.3. Let $\sigma^{-1}(\infty)$ and $\tau^{-1}(\infty)$ be two essential cusps of $G$. Let $m \in \Lambda_{\sigma}^{\wedge}-\{0\}$. The Poincaré series $P_{m, \sigma}(z, s)$ possesses at $\tau^{-1}(\infty)$ a Fourier expansion

$$
\left(\tau^{-1}\right)^{\prime}(z)^{k / 2} P_{m, \sigma}\left(\tau^{-1}(z), s\right)=\sum_{n \in q_{\tau}^{-1} \mathbb{Z}} F(n, \tau)(y) e\left(\left(n-\frac{\varkappa_{\tau}}{q_{\tau}}\right) x\right)
$$

with

$$
\begin{aligned}
& F(n, \tau)(y)=\delta_{\sigma, \tau} \delta_{m, n} e^{i k \pi} y^{s} \exp \left(-2 \pi\left|m-\frac{\varkappa_{\sigma}}{q_{\sigma}}\right| y\right)+ \\
& e^{i k \pi} y^{1-s} \sum_{c>0} c^{-2 s} K_{\sigma, \tau}(m, n, c) \frac{1}{q_{\tau}} \int_{-\infty}^{\infty} \frac{e^{-i k \arg (t+i)}}{\left(t^{2}+1\right)^{s}} e\left(-\left(m-\frac{\varkappa_{\sigma}}{q_{\sigma}}\right) \frac{t}{c^{2} y\left(t^{2}+1\right)}\right) \\
& \quad \times \exp \left(-2 \pi\left|m-\frac{\varkappa_{\sigma}}{q_{\sigma}}\right| \frac{1}{c^{2} y\left(t^{2}+1\right)}\right) e\left(-\left(n-\frac{\varkappa_{\tau}}{q_{\tau}}\right) y t\right) d t .
\end{aligned}
$$

Proposition 1.4. Let $\sigma^{-1}(\infty)$ and $\tau^{-1}(\infty)$ be two essential cusps of $G$. Let $m, n \in \Lambda_{\sigma}^{\wedge}-\{0\}$. Let $s, t \in \mathbb{C}$ with $\Re(s), \Re(t)>1$. Then the scalar product of the two Poincaré series $P_{m, \sigma}(z, s)$ and $P_{n, \tau}(z, t)$ is given by

$$
\begin{aligned}
\left\langle P_{m, \sigma}(\cdot, s), P_{n, \tau}(\cdot, t)\right\rangle= & \delta_{\sigma, \tau} \delta_{m, n} q_{\tau}\left(2 \pi\left(\left|m-\frac{\varkappa_{\sigma}}{q_{\sigma}}\right|+\left|n-\frac{\varkappa_{\tau}}{q_{\tau}}\right|\right)\right)^{1-s-\bar{t}} \Gamma(\bar{t}+s-1)+ \\
& \sum_{c>0} \frac{K_{\sigma, \tau}(m, n, c)}{c^{2 s}} \int_{0}^{\infty} y^{\bar{t}-s-1} \exp \left(-2 \pi y\left|n-\frac{\varkappa_{\tau}}{q_{\tau}}\right|\right) I(m, j, c, y) d y,
\end{aligned}
$$

with

$$
\begin{gathered}
I(m, j, c, y)= \\
\int_{-\infty}^{\infty} \frac{e^{-i k \arg (u+i)}}{\left(u^{2}+1\right)^{s}} e\left(\frac{-u\left(m-\frac{\varkappa_{\sigma}}{q_{\sigma}}\right)}{c^{2} y\left(u^{2}+1\right)}\right) \exp \left(\frac{-2 \pi\left|m-\frac{\varkappa_{\sigma}}{q_{\sigma}}\right|}{c^{2} y\left(u^{2}+1\right)}\right) e\left(-\left(n-\frac{\varkappa_{\tau}}{q_{\tau}}\right) y u\right) d u .
\end{gathered}
$$




\subsection{The Goldfeld-Sarnak method}

In this section we still work with a discrete subgroup $G$ of $\mathrm{SL}_{2}(\mathbb{R})$ and a multiplicative system $\psi$ of weight $k$. The goal is to obtain a first formula for the asymptotic behaviour of the function $K_{\sigma, \tau}(m, n, c)$. In analytic number theory, one possibility to prove the asymptotic behaviour for an arithmetic function $t(c)$, is to use the analytic properties of its zeta function $\sum t(c) c^{-s}$. In our case, the difficulty comes from the lack of information about the Selberg-Kloosterman zeta function $Z_{m, n}(s)$. However, the Kuznetsov formula for Kloosterman sums (which led to the formula (4) of Introduction), can also be developed for twisted Kloosterman sums and, as consequence of the complete summation formula, one obtains the asymptotic behaviour. All details were given by Proskurin in [36]. Nevertheless, it is possible to derive the desired properties of $Z_{m, n}(s)$, the most difficult being the growth condition in vertical strip; this was achieved by Goldfeld and Sarnak in a short and elegant paper (see [12]). In the case of the sums $K_{3}(m, n, c)$, it is still possible to apply such a method (see [31]) but since we shall need all spectral information, we shall have to deal with the complete formula. In the first part of this section we shall summarize the ideas of [12]; it consists in an estimate for the Laplacian, as well as the classical machinery build on the Poincaré series. In the second part, the asymptotic formula is derived in Theorem 1.2

\subsubsection{The Kloosterman-Selberg zeta function}

In the formula of Proposition 1.4 , the integral on the right side is given by

$$
\begin{aligned}
& \int_{0}^{\infty} y^{\bar{t}-s-1} \exp \left(-2 \pi y\left|n-\frac{\varkappa_{\tau}}{q_{\tau}}\right|\right) I(m, j, c, y) d y \\
& =\int_{0}^{\infty} \int_{-\infty}^{\infty} y^{\bar{t}-s-1} \exp \left(-2 \pi y\left|n-\frac{\varkappa_{\tau}}{q_{\tau}}\right|\right) \frac{e^{-i k \arg (u+i)}}{\left(u^{2}+1\right)^{s}} \\
& \quad \times e\left(\frac{-u\left(m-\frac{\varkappa_{\sigma}}{q_{\sigma}}\right)}{c^{2} y\left(u^{2}+1\right)}\right) \exp \left(\frac{-2 \pi\left|m-\frac{\varkappa_{\sigma}}{q_{\sigma}}\right|}{c^{2} y\left(u^{2}+1\right)}\right) e\left(-\left(n-\frac{\varkappa_{\tau}}{q_{\tau}}\right) y u\right) d u d y \\
& =\int_{0}^{\infty} \int_{-\infty}^{\infty} y^{\bar{t}-s-1} \exp \left(-2 \pi y\left|n-\frac{\varkappa_{\tau}}{q_{\tau}}\right|\right) \frac{e^{-i k \arg (u+i)}}{\left(u^{2}+1\right)^{s}} \\
& \quad \times\left\{e\left(\frac{-u\left(m-\frac{\varkappa_{\sigma}}{q_{\sigma}}\right)}{c^{2} y\left(u^{2}+1\right)}\right) \exp \left(\frac{-2 \pi\left|m-\frac{\varkappa_{\sigma}}{q_{\sigma}}\right|}{c^{2} y\left(u^{2}+1\right)}\right)-1\right\} e\left(-\left(n-\frac{\varkappa_{\tau}}{q_{\tau}}\right) y u\right) d u d y \\
& +\int_{0}^{\infty} \int_{-\infty}^{\infty} y^{\bar{t}-s-1} \exp \left(-2 \pi y\left|n-\frac{\varkappa_{\tau}}{q_{\tau}}\right|\right) \frac{e^{-i k \arg (u+i)}}{\left(u^{2}+1\right)^{s}} e\left(-\left(n-\frac{\varkappa_{\tau}}{q_{\tau}}\right) y u\right) d u d y .
\end{aligned}
$$

Denoting by $I_{1}(c)$ the first double integral (1.2.1) and by $I_{2}$ the second double integral (1.2.2), independent of $c$. We have shown that 


$$
\begin{aligned}
\left\langle P_{m, \sigma}(\cdot, s), P_{n, \tau}(\cdot, t)\right\rangle=\delta_{\sigma, \tau} \delta_{m, n} q_{\tau} & \left(2 \pi\left(\left|m-\frac{\varkappa_{\sigma}}{q_{\sigma}}\right|+\left|n-\frac{\varkappa_{\tau}}{q_{\tau}}\right|\right)\right)^{1-s-\bar{t}} \Gamma(\bar{t}+s-1) \\
& +\sum_{c>0} \frac{K_{\sigma, \tau}(m, n, c)}{c^{2 s}} I_{1}(c)+I_{2} Z_{\sigma, \tau}(m, n, s) .
\end{aligned}
$$

The estimation of $I_{2}$ is easy to handle.

Lemma 1.1. If $t=\bar{s}+2$, then

$$
I_{2}=\left|n-\frac{\varkappa_{\tau}}{q_{\tau}}\right|^{-2} \frac{e^{-i k \pi / 2}}{4 \pi} \frac{2^{-2 s} \Gamma(2 s+1)}{\Gamma\left(s+\frac{k}{2} \operatorname{sgn}\left(n-\frac{\varkappa_{\tau}}{q_{\tau}}\right)\right) \Gamma\left(s+2-\frac{k}{2} \operatorname{sgn}\left(n-\frac{\varkappa_{\tau}}{q_{\tau}}\right)\right)} .
$$

The goal is then to find an upper bound for $I_{1}(c)$, which makes the sum over the $c$ 's in 1.2.3 converge. In (1.2.1), we make appear the dependance in $c$ by using the estimate $\exp \left(z^{-1}\right)-1 \ll$ $z^{-1}$ whenever $z \gg 1$. Thus, introducing a constant $\alpha>0$ which we shall choose later, we have

$$
\begin{aligned}
& \left|I_{1}(c)\right| \leqslant \\
& \leqslant \int_{-\infty}^{\infty}\left(u^{2}+1\right)^{-s} \int_{0}^{\infty} y^{\bar{t}-s-1}\left|\exp \left(-2 i \pi \frac{(u-i)\left(m-\varkappa_{1}\right)}{c^{2} q y\left(u^{2}+1\right)}\right)-1\right| \exp \left(\frac{-2 \pi y\left(n-\varkappa_{2}\right)}{q}\right) d y d u \\
& \leqslant \int_{-\infty}^{\infty}\left(u^{2}+1\right)^{-s}\left\{\int_{0}^{\alpha} 2 y^{\bar{t}-s-1} d y+\int_{\alpha}^{\infty} \frac{y^{\bar{t}-s-1}}{c^{2} y \sqrt{u^{2}+1}} \exp \left(\frac{-2 \pi y\left(n-\varkappa_{2}\right)}{q}\right) d y\right\} d u \\
& \leqslant 2 \frac{\alpha \bar{t}-s}{\bar{t}-s} \int_{-\infty}^{\infty}\left(u^{2}+1\right)^{-s} d u+c^{-2} \int_{-\infty}^{\infty}\left(u^{2}+1\right)^{-s-\frac{1}{2}} \int_{\alpha}^{\infty} y^{\bar{t}-s-2} \exp \left(\frac{-2 \pi y\left(n-\varkappa_{2}\right)}{q}\right) d y d u
\end{aligned}
$$

The first integral converges for $\Re(s)>\frac{1}{2}$ and the second integral for $\Re(\bar{t}-s)>1$. From this we obtain that

$$
I_{1}(c) \ll \frac{1}{\Re(s)-\frac{1}{2}}\left(\alpha^{\Re(\bar{t}-s)}+c^{-2}\right) .
$$

We choose $\alpha=c^{-1}$ and, as in the Lemma 1.1 above, $t=\bar{s}+2$; combined with the trivial estimate for Kloosterman sums, it shows that

$$
\sum_{c>0} \frac{K_{\sigma, \tau}(m, n, c)}{c^{2 s}} I_{1}(c)
$$

is holomorphic in $\Re(s)>\frac{1}{2}$ and is bounded by $\left(\Re(s)-\frac{1}{2}\right)^{-1}$. We can reformulate this as follows:

$$
Z_{\sigma, \tau}(2 s)-4 \pi e^{i k \pi / 2}\left|n-\frac{\varkappa_{\tau}}{q_{\tau}}\right|^{2} \frac{\Gamma\left(s+\operatorname{sgn}(n) \frac{k}{2}\right) \Gamma\left(s+2-\operatorname{sgn}(n) \frac{k}{2}\right)}{2^{-2 s} \Gamma(2 s+1)}\left\langle P_{m, \sigma}(\cdot, s), P_{n, \tau}(\cdot, \bar{s}+2)\right\rangle
$$

is holomorphic in $\Re(s)>\frac{1}{2}$ and bounded by $\left(\Re(s)-\frac{1}{2}\right)^{-1}$. Therefore the possible poles of $Z_{\sigma, \tau}(s)$ are located at $s=2 s_{i}$, for $s_{i}$ an exceptional spectral parameter. Moreover, for $\Re(s)>\frac{1}{2}$, 
$P_{n, \tau}(\cdot, \bar{s}+2)$ is bounded, thus one will have all necessary properties of $Z_{\sigma, \tau}(m, n, c)$ once we possess an upper bound for the Poincaré series in the vertical strip $\frac{1}{2}<\Re(s)<1$. This is proved in [12], using the property (1.1.13). Namely, from the upper bound

$$
\left|R_{\lambda}\right| \leqslant \frac{1}{\operatorname{distance}(\lambda, \operatorname{spectrum}(\Delta)}
$$

one obtains

$$
\left\|P_{m, \sigma}(z, s)\right\|=\mathcal{O}\left(\left(\Re(s)-\frac{1}{2}\right)^{-2}\right) .
$$

The above discussion is gathered in the following proposition:

Proposition 1.5. Let $0<k<1$ and let $G, \psi, \sigma, \tau, m, n$ as above. Then The Kloosterman-Selberg zeta function $Z_{\sigma, \tau}(s)$ defined by

$$
Z_{\sigma, \tau}(s)=\sum_{c>0} \frac{K_{\sigma, \tau}(m, n, c)}{c^{s}}
$$

has the following properties:

- holomorphy in $\Re(s)>2$,

- meromorphy in $\Re(s) \geqslant 1$, with polynomial growth

$$
Z_{\sigma, \tau}(s)=\mathcal{O}\left(\frac{|s|}{\Re(s)-1}\right),
$$

- poles at $s=2 s_{i}$, with residue

$$
\begin{aligned}
\operatorname{Res}_{s=2 s_{i}} Z_{\sigma, \tau}(s)= & e^{i k \pi / 2} \frac{4^{1-s_{i}}}{\pi^{2 s_{i}-1 / 2}} q_{\sigma} q_{\tau}\left(\left|n-\frac{\varkappa_{\tau}}{q_{\tau}}\right|\left|m-\frac{\varkappa_{\sigma}}{q_{\sigma}}\right|\right)^{3 / 4-s_{i}} \\
& \Gamma\left(2 s_{i}-1\right) \frac{\Gamma\left(s_{i}+\operatorname{sgn}(n) \frac{k}{2}\right)}{\Gamma\left(s_{i}-\operatorname{sgn}(m) \frac{k}{2}\right)} \sum_{u \in \mathcal{B}(\text { Mod })} \overline{a_{u}(\sigma, m)} a_{u}(\tau, n),
\end{aligned}
$$

where $\mathcal{B}(\mathrm{Mod})$ is an orthonormal basis of $\mathrm{L}_{\mathrm{Mod}}^{2}(\Gamma \backslash \mathbb{H}, k, \psi)$.

Proof. Everything has been already proved above, except the last statement about the residues, that we prove using Proposition 1.2 and Propositon 1.1

\subsubsection{Asymptotic behaviour}

The goal of this section is to derive an asymptotic formula for the function $K_{\sigma, \tau}(m, n, c)$ from the analytic properties of its zeta function $Z_{m, n}(s)$. This argument can be found in [12] or in [31] in the case of cubic Kloosterman sums $K_{3}(m, n, c)$. We write the exceptional spectral parameters as $s_{1}>s_{2}>\ldots>\frac{1}{2}$.

Theorem 1.2. Let $0<k<1$ and let $G, \psi, \sigma, \tau, m, n$ as above. Then for any $\varepsilon>0$,

$$
\sum_{0<c<X} \frac{K_{\sigma, \tau}(m, n, c)}{c^{\beta}} \sim \frac{\operatorname{Res}_{s=2 s_{1}} Z_{\sigma, \tau}(s)}{2 s_{1}-\beta} X^{2 s_{1}-\beta}+\mathcal{O}\left(X^{\max \left(2 s_{2}, 5 / 4+\varepsilon\right)-\beta}\right)
$$


Proof. Let $\alpha \in] 0,1\left[\right.$ and let $\omega_{1}>\alpha+1$ and $\omega_{2}>\alpha+2$; consider the counterclockwise integral of $Z(s-\alpha) \frac{X^{s}}{s(s-1)}$ around the rectangle with vertices $\omega_{1}-i T, \omega_{1}+i t, \omega_{2}+i T$ and $\omega_{2}-i T$. From the Phragmén-Lindelhöf Theorem and Proposition 1.5 one has $Z_{\sigma, \tau}(s)=\mathcal{O}\left(\Im(s)^{\Phi(s)}\right)$, for a linear function $\Phi$ satisfying $\Phi(1+\varepsilon)=1$ and $\Phi(2+\varepsilon)=0$. On the one side, as $T$ goes to infinity, it remains the integral on the vertical lines $\left(\omega_{1}\right)$ and $\left(\omega_{2}\right)$. On the other side, by the Cauchy theorem, this is equal to $\sum_{s_{i}} R_{i}$. Thus,

$\frac{1}{2 i \pi} \int_{\left(\omega_{2}\right)} Z(s-\alpha) \frac{X^{s}}{s(s-1)} d s=\sum_{s_{i}} R_{i} \frac{X^{2 s_{i}+\alpha}}{\left(2 s_{i}+\alpha\right)\left(2 s_{i}+\alpha-1\right)}+\frac{1}{2 i \pi} \int_{\left(\omega_{1}\right)} Z(s-\alpha) \frac{X^{s}}{s(s-1)} d s$,

where the sum on the right hand side is taken over the exceptional spectral parameter $s_{1}>s_{2}>$ $\ldots>s_{i}>\ldots>\frac{1}{2}$ and

$$
R_{i}=\operatorname{Res}_{s=2 s_{i}+\alpha}\left(Z_{\sigma, \tau}(s-\alpha)\right) .
$$

As $\omega_{2}>\alpha+2$, the left hand side converges, and we can interchange integral and summation. We obtain

$$
\begin{aligned}
\frac{1}{2 i \pi} \int_{\left(\omega_{2}\right)} Z(s-\alpha) \frac{X^{s}}{s(s-1)} d s & =\sum_{0<c} K_{\sigma, \tau}(m, n, c) c^{\alpha} \frac{1}{2 i \pi} \int_{(\beta)} \frac{(X / c)^{s}}{s(s-1} d s \\
& =\sum_{0<c \leqslant X} \frac{K_{\sigma, \tau}(m, n, c)}{c^{1-\alpha}}(X-c) .
\end{aligned}
$$

On the right side, the integral over $\left(\omega_{1}\right)$ is bounded by $X^{\omega_{1}}$. Comparing both sides of the equality gives

$$
\sum_{0<c<X} \frac{K_{\alpha_{1}, \alpha_{2}}(m, n, c)}{c^{1-\alpha}}(X-c)=\sum_{s_{i}} R_{i} \frac{X^{2 s_{i}+\alpha}}{\left(2 s_{i}+\alpha\right)\left(2 s_{i}+\alpha-1\right)}+X^{\omega_{1}} .
$$

We now differentiate this equation. Let $1 \ll \Delta X \ll X$. Substracting $(X)$ from $(X+\Delta X)$, we obtain

$$
\begin{gathered}
\sum_{0<c<X} \frac{K_{\alpha_{1}, \alpha_{2}}(m, n, c)}{c^{1-\alpha}} \Delta X+\sum_{X \leq c<X+\Delta X} \frac{K_{\alpha_{1}, \alpha_{2}}(m, n, c)}{c^{1-\alpha}}(X+\Delta X-c) \\
=\sum_{s_{i}} R_{i} \frac{(X+\Delta X)^{2 s_{i}+\alpha}-X^{2 s_{i}+\alpha}}{\left(2 s_{i}+\alpha\right)\left(2 s_{i}+\alpha-1\right)}+\mathcal{O}\left(X^{\omega_{1}}\right)
\end{gathered}
$$

The second sum on the link hand side is bounded by $\mathcal{O}\left(X^{\alpha-1 / 2}(\Delta X)^{2}\right)$. The term corresponding to each $s_{i}$ in the right hand side is equal to

$$
\frac{R_{i}}{2 s_{i}+\alpha-1} X^{2 s_{i}+\alpha-1} \Delta X+\mathcal{O}\left(X^{2 s_{i}+\alpha-2}(\Delta X)^{2}\right) .
$$

Dividing by $\Delta X$, we obtain

$$
\sum_{0<c<X} \frac{K_{\alpha_{1}, \alpha_{2}}(m, n, c)}{c^{1-\alpha}}=\frac{R_{1}}{2 s_{1}+\alpha-1} X^{2 s_{1}+\alpha-1}+\mathcal{O}\left(X^{\alpha-1 / 2} \Delta X\right)+\mathcal{O}\left(X^{2 s_{2}+\alpha-1}+X^{\omega_{1}} \Delta X^{-1}\right) .
$$


Choose $\omega_{1}=\alpha+1+\varepsilon$; then $\Delta X$ has to be chosen equal to $X^{3 / 4}$, and, writing $\beta=1-\alpha$, we obtain

$$
\sum_{0<c<X} \frac{K_{\alpha_{1}, \alpha_{2}}(m, n, c)}{c^{\beta}}=\frac{R_{1}}{2 s_{1}-\beta} X^{2 s_{1}-\beta}+\mathcal{O}\left(X^{\max \left(2 s_{2}, 5 / 4+\varepsilon\right)-\beta}\right) .
$$

This finishes the proof of Theorem 1.2 .

Remark 1.3. We know that some spectral gap occurs in the exceptional spectrum. For example, Goldfeld and Sarnak (see [39], Theorem 3.6) proved that when $G=\Gamma_{0}(4 N), k=\frac{1}{2}$ and $\psi$ is the multiplicative factor associated to the theta function (see next section) then $s_{2} \leqslant \frac{5}{8}$. 


\subsection{Theta functions of half-integral weight}

In this section, we shall restrict ourselves to congruence subgroups $\Gamma_{0}(N)$ of $\mathrm{SL}_{2}(\mathbb{R})$, to the weight $k=\frac{1}{2}$, and to factor systems $\psi$ of the shape $\kappa \chi$, where $\kappa$ is defined in (1.3.5) and $\chi$ is a primitive Dirichlet character. Under these conditions, we shall give an explicit orthonormal basis of the modular forms, i.e. of the minimal eigenspace of Maaß forms; this will then allow us to determine explicitly the right hand side of the formula in Theorem 1.2. The main ingredient will be the classical theta function, and we start with some facts about it.

It is known that the function $\theta(z)=\sum_{n \in \mathbb{Z}} e^{i \pi n^{2} z}$ satisfies (always with the choice of the argument of a complex number in $[0,2 \pi[)$

$$
\begin{array}{r}
\theta(z+2)=\theta(z) \\
\theta(z)=\sqrt{\frac{i}{z}} \theta\left(\frac{-1}{z}\right) .
\end{array}
$$

By Remark 1.1 of Section 1.1, this implies a modularity property of $\theta$ for the group $\Lambda=\left\langle T^{2}, S\right\rangle$, where

$$
T=\left(\begin{array}{ll}
1 & 1 \\
0 & 1
\end{array}\right) \quad \text { et } \quad S=\left(\begin{array}{cc}
0 & -1 \\
1 & 0
\end{array}\right) .
$$

Proposition 1.6. Let $\gamma \in \Lambda$. There exists a function $\kappa_{\theta}$ on $\Lambda$ such that

$$
\gamma^{\prime}(z)^{1 / 4} \theta(\gamma(z))=\kappa_{\theta}(\gamma) \theta(z) \quad \forall z \in \mathbb{H}, \gamma \in \Lambda .
$$

Because of $\kappa_{\theta}(-I d)=1, \kappa_{\theta}$ is determined by its values on the elements $\gamma=\left(\begin{array}{ll}a & b \\ c & d\end{array}\right) \in \Lambda$, with $d>0$; on such an element, it holds

$$
\kappa_{\theta}(\gamma)= \begin{cases}\left(\frac{2 b}{d}\right)_{2} \varepsilon_{d} \begin{cases}i & \text { if } c>0 \\ 1 & \text { if } c \leq 0\end{cases} & \text { for } c \text { even and } b \neq 0, \\ \begin{cases}i & \text { if } c>0 \\ 1 & \text { if } c \leq 0\end{cases} & \text { for } c \text { even and } b=0, \\ e^{\frac{i \pi}{4}}\left(\frac{2 a}{c}\right)_{2} \varepsilon_{c} & \text { for } c \text { odd and } a \neq 0, \\ e^{\frac{i \pi}{4}} & \text { for } c \text { odd and } a=0,\end{cases}
$$

where $\varepsilon_{x}=1$ if $x \equiv 1(\bmod 4)$ and $\varepsilon_{x}=i$ if $x \equiv 3(\bmod 4)$.

Proof. This property of $\kappa_{\theta}$ on $\Lambda$ was proved by Kubota in [25]. This result is in fact considerably older; actually, it was proved by Hermite (see [16]) and then by Weber (see [44] $§ 23-\S 28$ ). Our result is different from the result of [25], because of the choice of the branch of $g^{\prime}(z)^{1 / 4}$.

This makes from $\theta$ a modular form of weight $\frac{1}{2}$ for the group $\Lambda$. For convenience, we wish to work in the context of congruence subgroups; for it we define

$$
\vartheta(z)=\theta(2 z) .
$$

If we define, for an element $\gamma=\left(\begin{array}{ll}a & b \\ c & d\end{array}\right)$, an element $\gamma_{t}$ by $\gamma_{t}=\left(\begin{array}{cc}a & t b \\ c / t & d\end{array}\right)$, then 


$$
\begin{aligned}
\gamma^{\prime}(z)^{1 / 4} \vartheta(\gamma(z)) & =\gamma_{2}^{\prime}(2 z)^{1 / 4} \theta\left(\gamma_{2}(2 z)\right) \quad \forall \gamma \in \Gamma_{0}(4) \\
& =\kappa_{\theta}\left(\gamma_{2}\right) \vartheta(z) \quad \text { because } \gamma_{2} \in \Lambda
\end{aligned}
$$

One sees then that $\gamma \mapsto \kappa_{\theta}\left(\gamma_{2}\right)=: \kappa(\gamma)$ is a multiplicative system for the group $\Gamma_{0}(4)$ and for the weight $\frac{1}{2}$. The result of the last proposition gives then, for $\gamma \in \Gamma_{0}(4)$,

$$
\kappa(\gamma)= \begin{cases}\left(\frac{b}{d}\right)_{2} \varepsilon_{d} \begin{cases}i & \text { if } c>0 \\ 1 & \text { if } c \leq 0\end{cases} & \text { for } c \text { even and } b \neq 0, \\ \begin{cases}i & \text { if } c>0 \\ 1 & \text { if } c \leq 0\end{cases} & \text { for } c \text { even and } b=0 .\end{cases}
$$

Thus $\vartheta$ is an element of $\mathrm{L}_{\text {Mod }}^{2}\left(\Gamma_{0}(4 N), 1 / 2, \kappa \chi\right)$. By Proposition 1.1 of Section 1.1.1, we know that $y^{1 / 4} \vartheta$ is a non cuspidal Maaß form of eigenvalue $3 / 16$, which is the smallest possible, by the formula (1.1.8).

Recall also that for any Dirichlet character $\chi$ modulo $4 N, \kappa \chi$ can be made as a multiplicative system for $\Gamma_{0}(4 N)$, by defining $\chi(\gamma)$ as $\chi(d)$, if $\gamma=\left(\begin{array}{ll}a & b \\ c & d\end{array}\right)$. This is in particular true with any Dirichlet character $\chi$ modulo $f$, with $f \mid N$. The main result of the last section, Theorem 1.2 , can be applied in this context. It gives the following theorem:

Theorem 1.3. Let $\chi$ be a primitive Dirichlet character modulo $f$. Let $\sigma^{-1}(\infty)$ and $\tau^{-1}(\infty)$ be two essential cusps of $\Gamma_{0}(4 N)$, and let $m \in \Lambda_{\sigma}^{-1}-\{0\}$ and $n \in \Lambda_{\tau}^{-1}-\{0\}$. Let $K_{\sigma, \tau}(m, n, c)$ be the Kloosterman sum associated to the multiplicative system $\kappa \chi$. Then, if $N$ is an integer so that $f \mid N$, we have

$X^{-3 / 2} \sum_{c \leqslant X} K_{\sigma, \tau}(m, n, c) \sim \frac{2}{3} \frac{1+i}{\sqrt{\pi}} q_{\sigma} q_{\tau}\left\{\begin{array}{ll}\sqrt{\pi}^{-\operatorname{sgn}(m)} & \text { if } \operatorname{sgn}(m)=\operatorname{sgn}(n) \\ 1 & \text { if } \operatorname{sgn}(m) \neq \operatorname{sgn}(n)\end{array} \sum_{u} \overline{a_{u}(\sigma, m)} a_{u}(\tau, n)\right.$,

where $u$ belongs to an orthonormal basis of the space $\mathrm{L}_{\text {Mod }}^{2}\left(\Gamma_{0}(4 N), 1 / 2, \kappa \chi\right)$ and $a_{u}(\cdot, \cdot)$ are defined by 1.1.5.

The rest of this section is devoted to find an orthonormal basis of $\mathrm{L}_{\text {Mod }}^{2}\left(\Gamma_{0}(4 N), 1 / 2, \kappa \chi\right)$. We introduce theta function twisted by a Dirichlet character,

$$
\vartheta_{\chi}(z)=\sum_{n} \chi(n) e\left(n^{2} z\right)
$$

We remark that, with the notations of (1.1.5), $a_{\vartheta_{\chi}}(I d, 0)=0$. This will be needed latter on.

\subsubsection{A result of Serre and Stark}

One of the main results of Serre and Stark $([42])$ is the fact that, for any Dirichlet character $\chi$, the space of modular forms $\operatorname{Mod}\left(\Gamma_{0}\left(4 \operatorname{cond}(\chi)^{2}\right), \chi, \frac{1}{2}\right)$ only contains one newform, $\vartheta_{\chi}$. From this, they deduce that there exists a basis of $\operatorname{Mod}\left(\Gamma_{0}(4 N), \chi, \frac{1}{2}\right)$ formed by theta functions. Before to state this result, let us introduce some notations. 
In the theory of half-integral modular forms, one has non trivial Hecke operators, not for any prime $p$, but for squares $p^{2}$. The Hecke operators $T_{p^{2}}$ are a defined on $\operatorname{Mod}\left(\Gamma_{0}(4 N) \backslash \mathbb{H}, \frac{1}{2}, \kappa, \chi\right)$. Their action on a modular form

$$
f(z)=\sum_{n=0}^{\infty} a_{f}(n) e(n z)
$$

is given by

$$
T_{p^{2}} f(z)=\sum_{n=0}^{\infty} b_{f}(n) e(n z)
$$

with

$$
b_{f}(n)= \begin{cases}a_{f}\left(n p^{2}\right) & \text { if } p \mid 2 N, \\ a_{f}\left(n p^{2}\right)+\frac{\chi(p)}{p}\left(\frac{n}{p}\right) a_{f}(n)+\frac{\chi^{2}(p)}{p} a_{f}\left(n / p^{2}\right) & \text { if } p \nmid 2 N .\end{cases}
$$

A useful property of the Hecke operators is that $\bar{\chi}(p) T_{p^{2}}$ is hermitian; if $p \nmid 2 N$, then

$$
\left\langle f \mid T_{p^{2}}, g\right\rangle=\chi^{2}(p)\left\langle f, g \mid T_{p^{2}}\right\rangle .
$$

The function $\theta_{\chi}$ is an eigenfunction of any operator $T_{p^{2}}$ for $p \nmid 2 N$ of eigenvalue $\chi(p)\left(1+p^{-1}\right)$.

For $t$ odd, the Kronecker symbol $\chi_{t}$ is the Dirichlet character $n \mapsto\left(\frac{t}{n}\right)_{2}$ of conductor $t$ or $4 t$ according to whether $t \equiv 1(\bmod 4)$ or $t \equiv 3(\bmod 4)$. Now let us define an operator

$$
\begin{aligned}
V_{t}: \operatorname{Mod}\left(\Gamma_{0}(4 N), \chi, \frac{1}{2}\right) & \longrightarrow \operatorname{Mod}\left(\Gamma_{0}(4 N t), \chi \chi_{t}, \frac{1}{2}\right) . \\
f(z) & \mapsto f(t z) .
\end{aligned}
$$

Then, $V_{t}$ and $T_{p^{2}}$ commute if $p \nmid t$.

To any Dirichlet character $\chi$ of modulus $N$, there is an associated primitive Dirichlet character $\chi^{\prime}$ of modulus the conductor of $\chi$, written $f_{\chi}$; for two Dirichlet characters $\chi_{1}$ and $\chi_{2}$, when we write $\chi_{1} \chi_{2}$ we always mean the primitive Dirichlet character associated to the product of $\chi_{1}$ and $\chi_{2}$.

Finally, we recall the definition of newform and oldform. Let $f \in \operatorname{Mod}\left(\Gamma_{0}(4 N) \backslash \mathbb{H}, \frac{1}{2}, \kappa \chi\right)$ be an eigenform of all but finitely many $T_{p^{2}}$. We say that $f$ is an oldform if there exists some prime $p \mid N$ such that, either $f \in \operatorname{Mod}\left(\Gamma_{0}(4 N / p) \backslash \mathbb{H}, \frac{1}{2}, \kappa \chi\right)$, or $f=V_{p} g$, for some $g \in \operatorname{Mod}\left(\Gamma_{0}(4 N / p) \backslash \mathbb{H}, \frac{1}{2}, \kappa \chi \chi_{p}\right)$; if $f$ is not an oldform, it is said to be a newform. Let $\operatorname{New}\left(\Gamma_{0}(4 N) \backslash \mathbb{H}, \frac{1}{2}, \kappa \chi\right)$ be the space spanned by newform. Then Serre and Stark proved ([42], Theorem 3) that New $\left(\Gamma_{0}(4 \operatorname{cond}(\chi)) \backslash \mathbb{H}, \frac{1}{2}, \kappa \chi\right)$ is one dimensional, generated by $\vartheta_{\chi}$. This allowed them to prove that any modular form of halfintegral weight is a combination of theta series.

Theorem 1.4. Let $N \in \mathbb{N}$. Let $\chi$ be a primitive Dirichlet character modulo $N$. A basis of the space $\operatorname{Mod}\left(\Gamma_{0}(4 N) \backslash \mathbb{H}, \frac{1}{2}, \kappa \chi\right)$ is given by the family

$$
V_{d u^{2}} \vartheta_{\chi \chi_{d}}
$$

where $d$ and $u$ are submitted to the conditions

$$
\begin{cases}d \text { square-free and } f(d)^{2} d \mid N & (D) \\ u^{2} \mid \frac{N}{f(d)^{2} d} & \left(D_{d}\right) .\end{cases}
$$


Proof. This is a reformulation of Theorem A of [42], which states that a basis of the space $\operatorname{Mod}\left(\Gamma_{0}(4 N) \backslash \mathbb{H}, \kappa \chi, \frac{1}{2}\right)$ is given by the family $\left\{V_{t} \vartheta_{\psi}\right\}_{(\psi, t)}$, where

$$
\begin{aligned}
& \text { (i) } \psi \text { is a primitive Dirichlet character, } \\
& \text { (ii) } \left.\psi \chi_{t}=\chi, \quad \text { (as group homomorphisms on } \Gamma_{0}(4 N)\right), \\
& \text { (iii) }\left(f_{\psi}\right)^{2} t \mid N .
\end{aligned}
$$

Replacing the condition (ii) by $\psi=\chi \chi_{t}$, we see that (iii) is equivalent with $\left(f_{\chi \chi_{t}}\right)^{2} t \mid N$. Let us decompose $t=d u^{2}$, with $d$ square-free; then $\chi_{t}=\chi_{d}$, and as $\chi$ is now fixed, we simplify notations by writing $f(t)$ for $f_{\chi \chi_{t}}$, the conductor of $\chi_{\chi_{t}}$.

Corollary 1.1. Let $D, f$ be odd positive integers. Let $\chi$ be a primitive Dirichlet character of conductor $f$. The space $\operatorname{Mod}\left(\Gamma_{0}(4 D f), \kappa \chi, \frac{1}{2}\right)$ is non-empty only if $\chi=\chi_{f}$ with $f \equiv 1(\bmod 4)$. Any d satisfying $(D)$ has to be a multiple of $f$, say $d=f t$, and $\chi \chi_{t f}=\chi_{t}$.

Proof. Let $f=\prod p_{i}^{f_{i}}, p_{i}$ odd. Let $d$ be such that $4 \operatorname{cond}\left(\chi \chi_{d}\right)^{2} d \mid 4 D f$. Then $d$ has to be odd. We then use the product decomposition

$$
\chi=\prod \chi_{i},
$$

with $\chi_{i}$ a character of conductor $p_{i}^{f_{i}}$. Also, we have

$$
\chi_{d}=\epsilon \prod_{p \mid d}\left(\frac{\cdot}{p}\right)_{2},
$$

where $\epsilon$ is the trivial character if $d \equiv 1(\bmod 4)$ and $\epsilon$ is the non trivial character modulo 4 if $d \equiv 3(\bmod 4)$. Then,

$$
\chi_{d}=\epsilon \prod_{p_{i}} \chi_{i} \prod_{p \mid d} \chi_{p} .
$$

If some $p_{i}$ does not divide $d$, then $p_{i}^{f_{i}} \mid \operatorname{cond}\left(\chi \chi_{d}\right)$ and therefore $p_{i}^{2 f_{i}} \operatorname{divides} D f ; \operatorname{but} f_{i}=\operatorname{ord}_{p_{i}}(f)=$ $\operatorname{ord}_{p_{i}}(D f)$. Thus all $p_{i}$ divide $d$. Moreover, $\operatorname{cond}\left(\chi_{i} \chi_{p_{i}}\right)=p_{i}^{f_{i}}$, except if $\chi_{i}=\chi_{p_{i}}$, and we obtain the same contradiction as previously, if some $\chi_{i} \neq \chi_{p_{i}}$; thus, $\chi=\chi_{f}$. In particular, $f$ is squarefree and $f$ divides $d$. As we work with even characters, we need $f \equiv 1(\bmod 4)$. Let us write $d=f t$, with $t$ square-free and $\operatorname{gcd}(t, f)=1$. Then,

$$
\chi \chi_{d}=\epsilon \prod_{p_{i}} \chi_{i} \prod_{p|d, p| f} \chi_{p} \prod_{p|d, p| t} \chi_{p}
$$

and the primitive character associated to $\chi \chi_{d}$ is

$$
\chi \chi_{d}=\epsilon \prod_{p|d, p| t} \chi_{p}
$$

but as the conductor has to be odd, we need $d \equiv 1(\bmod 4)$ i.e. $t \equiv f \equiv 1(\bmod 4)$. One obtains therefore $\chi_{d}=\chi_{t}$, of conductor $t$.

We remark that the argument used in this proof is no more valid if we consider the larger space $\operatorname{Mod}\left(\Gamma_{0}\left(4 D f^{i}\right), \kappa \chi, \frac{1}{2}\right)$, for some $i \geqslant 2$. 


\subsubsection{Orthonormalisation of theta functions}

Among the set of theta functions $V_{d u^{2}} \vartheta_{\chi \chi_{d}}$ forming a basis of $\operatorname{Mod}\left(\Gamma_{0}(4 N) \backslash \mathbb{H}, \frac{1}{2}, \kappa \chi\right)$ (see Theorem 1.4), some are orthogonal and some are not. The following lemmas describe precisely the scalar product of two theta functions.

Lemma 1.2. Let $d$ and $d^{\prime}$ satisfy $(D)$, and let $u$ and $u^{\prime}$ satisfy $\left(D_{d}\right)$ and $\left(D_{d^{\prime}}\right)$, respectively. Assume $d \neq d^{\prime}$. Then $\left\langle V_{d u^{2}} \vartheta_{\chi \chi_{d}}, V_{d^{\prime} u^{\prime 2}} \vartheta_{\chi \chi_{d^{\prime}}}\right\rangle=0$.

Proof. Since $d$ and $d^{\prime}$ are square-free, we can choose $p \nmid 2 N$ such that $\chi_{d}(p) \neq \chi_{d^{\prime}}(p)$. We use the fact that $\vartheta_{\chi \chi_{d}}$ belongs to $\operatorname{New}\left(4 f(d)^{2}, \chi \chi_{d}\right)$, and is an eigenfunction of $T_{p^{2}}$ with eigenvalue $\chi \chi_{d}(p)\left(1+p^{-1}\right)$. The operators $V_{d u^{2}}$ and $T_{p^{2}}$ commute, thus $V_{d u^{2}} \vartheta_{\chi \chi_{d}}$ is an eigenfunction of $T_{p^{2}}$, for the same eigenvalue. Then,

$$
\begin{aligned}
& \bar{\chi}(p) \chi \chi_{d}(p)\left(1+p^{-1}\right)\left\langle V_{d u^{2}} \vartheta_{\chi \chi_{d}}, V_{d^{\prime} u^{\prime 2}} \vartheta_{\chi \chi_{d}^{\prime}}\right\rangle=\left\langle T_{p^{2}} \bar{\chi}(p) V_{d u^{2}} \vartheta_{\chi \chi_{d}}, V_{d^{\prime} u^{\prime 2}} \vartheta_{\chi \chi_{d}^{\prime}}\right\rangle \\
= & \left\langle V_{d u^{2}} \vartheta_{\chi \chi_{d}}, T_{p^{2}} \bar{\chi}(p) V_{d^{\prime} u^{\prime 2}} \vartheta_{\chi \chi_{d}^{\prime}}\right\rangle=\chi(p) \overline{\chi \chi_{d^{\prime}}}(p)\left(1+p^{-1}\right)\left\langle V_{d u^{2}} \vartheta_{\chi \chi_{d}}, V_{d^{\prime} u^{\prime 2}} \vartheta_{\chi \chi_{d}^{\prime}}\right\rangle .
\end{aligned}
$$

On the one side, $\bar{\chi}(p) \chi \chi_{d}(p)=\chi_{d}(p)$, and on the other side, $\chi(p) \overline{\chi \chi_{d^{\prime}}}(p)=\chi_{d^{\prime}}(p) \neq \chi_{d}(p)$, as $\chi_{d}(p)$ is real. (One remarks that we used three different Hecke operators $T_{p^{2}}$, each one being defined according to a different character.)

Let now $d$ be a fixed integer, satisfying the condition $(D)$. We study the set of functions $V_{d u^{2}} \theta_{\chi \chi_{d}}$, where $u$ satisfies the condition $\left(D_{d}\right)$ and compute the scalar product by the following lemma.

Lemma 1.3 (Rankin-Selberg). Let $\Gamma$ be a subgroup of $\mathrm{SL}_{2}(\mathbb{Z})$. Let $E(z, s)=\sum_{\gamma \in \Gamma_{\infty} \backslash \Gamma} \Im(\gamma z)^{s}$. Let $f, g \in \operatorname{Mod}(\Gamma, k, \chi)$, and denote by $a_{f}(n)$ and $a_{g}(n)$ their Fourier coefficients at $\infty$. Assume that $a_{f}(0) a_{g}(0)=0$. Then

$$
\langle f, g E(\cdot, s)\rangle=\Gamma(s+k-1) \sum_{n>0} \frac{a(n) \overline{b(n)}}{(4 \pi n)^{k+s-1}} .
$$

Proof. It is the usual unfolding method, once one remarks that $f(z) \bar{g}(z) \Im(z)$ is $\Gamma$-invariant.

Define $\frac{N}{f(d)^{2} d}=X$, and define a divisor $X_{d}$ of $X$ as $X_{d}=\prod_{p_{i} \mid f(d)} p_{i}^{e_{i}}$, where $X=\prod p_{i}^{e_{i}}$; $X_{d}$ will be said to be the divisor of $X$ supported by $f(d)$.

Lemma 1.4. With the notations as above, let $d$ satisfy Condition $(D)$ and let $u$ and $u^{\prime}$ satisfy Condition $\left(D_{d}\right)$, i.e. $u^{2} \mid X$ and $u^{\prime 2} \mid X$. Denote by $s$ and $s^{\prime}$, the divisors of $u$ and $u^{\prime}$, respectively, supported by $f(d)$. Then

$$
\left\langle V_{d u^{2}} \vartheta_{\chi \chi_{d}}(z), V_{d u^{\prime 2}} \vartheta_{\chi \chi_{d}}(z)\right\rangle= \begin{cases}0 & \text { if } s \neq s^{\prime}, \\ C(N, d) \chi \chi_{d}\left(\frac{u^{\prime}}{s^{\prime}}\right) \overline{\chi \chi_{d}}\left(\frac{u}{s}\right) \frac{\operatorname{gcd}\left(u, u^{\prime}\right)}{u u^{\prime}} & \text { if } s=s^{\prime},\end{cases}
$$

where we defined the constant

$$
C(N, d)=\frac{\pi N}{2 \sqrt{d}} \prod_{p \mid N} \frac{p+1}{p} \prod_{p \mid f(d)} \frac{p-1}{p} .
$$


Proof. Lemma 1.3 applied with $k=\frac{1}{2}$ and $\Gamma=\Gamma_{0}(4 N)$ gives

$$
\left\langle V_{d u^{2}} \vartheta_{\chi \chi_{d}}, V_{d u^{\prime 2}} \vartheta_{\chi \chi_{d}} E(\cdot, s)\right\rangle=\Gamma\left(s-\frac{1}{2}\right) \sum_{n} \frac{a(n) \overline{a^{\prime}(n)}}{(4 \pi n)^{s-\frac{1}{2}}},
$$

with

$$
a(n)=\left\{\begin{array}{ll}
\chi \chi_{d}\left(\sqrt{\frac{n}{d u^{2}}}\right) & \text { if } n \in d u^{2} \mathbb{Z}^{2}, \\
0 & \text { otherwise, }
\end{array} \quad \text { and } \quad a^{\prime}(n)= \begin{cases}\chi \chi_{d}\left(\sqrt{\frac{n}{d u^{\prime 2}}}\right) & \text { if } n \in d u^{\prime 2} \mathbb{Z}^{2} \\
0 & \text { otherwise. }\end{cases}\right.
$$

Let $g=\operatorname{gcd}\left(u, u^{\prime}\right)$; then $n$ has to belong to $d \frac{u^{2} u^{\prime 2}}{g^{2}} \mathbb{Z}^{2}$, and we obtain

$$
\begin{aligned}
& \left\langle V_{d u^{2}} \vartheta_{\chi \chi_{d}}, V_{d u^{\prime 2}} \vartheta_{\chi \chi_{d}} E(\cdot, s)\right\rangle=\Gamma\left(s-\frac{1}{2}\right) \sum_{m} \frac{\chi \chi_{d}\left(\frac{u^{\prime} m}{g}\right) \overline{\chi \chi_{d}\left(\frac{u m}{g}\right)}}{\left(\frac{4 \pi d m^{2} u^{2} u^{\prime 2}}{g^{2}}\right)^{s-\frac{1}{2}}} \\
& =\Gamma\left(s-\frac{1}{2}\right) \chi \chi_{d}\left(\frac{u^{\prime}}{g}\right) \overline{\chi \chi_{d}}\left(\frac{u}{g}\right)\left(\frac{4 \pi d u^{2} u^{\prime 2}}{g^{2}}\right)^{1 / 2-s} \sum_{n} \frac{\chi \chi_{d}(n) \overline{\chi \chi_{d}(n)}}{\left(n^{2}\right)^{s-\frac{1}{2}}} \\
& =\Gamma\left(s-\frac{1}{2}\right) \chi \chi_{d}\left(\frac{u^{\prime}}{g}\right) \overline{\chi \chi d}\left(\frac{u}{g}\right)\left(\frac{4 \pi d u^{2} u^{\prime 2}}{g^{2}}\right)^{1 / 2-s} \sum_{\substack{n>0 \\
(n, f(d))=1}} \frac{1}{\left(n^{2}\right)^{s-\frac{1}{2}}} .
\end{aligned}
$$

Taking the residue at $s=1$, we get

$$
\begin{gathered}
\left\langle V_{d u^{2}} \vartheta_{\chi \chi_{d}}, V_{d u^{\prime 2}} \vartheta_{\chi \chi_{d}}\right\rangle \\
=\left(\operatorname{Res}_{s=1} E(z, s)\right)^{-1} \chi \chi_{d}\left(\frac{u^{\prime}}{g}\right) \overline{\chi \chi_{d}}\left(\frac{u}{g}\right) \frac{g}{2 u u^{\prime} \sqrt{d}} \operatorname{Res}_{s=1}\left(\sum_{(n, f(d))=1} \frac{1}{\left(n^{2}\right)^{s-\frac{1}{2}}}\right) \\
=\left(\operatorname{Res}_{s=1} E(z, s)\right)^{-1} \chi \chi_{d}\left(\frac{u^{\prime}}{g}\right) \overline{\chi \chi_{d}}\left(\frac{u}{g}\right) \frac{g}{4 u u^{\prime} \sqrt{d}} \prod_{p \mid f(d)} \frac{p-1}{p} .
\end{gathered}
$$

Let $s, s^{\prime}$ and $g_{d}$ be the divisors of $u, u^{\prime}$ and $g$, respectively, with support in $f(d)$. Then, $\chi \chi_{d}\left(\frac{u^{\prime}}{g}\right) \overline{\chi \chi_{d}}\left(\frac{u}{g}\right) \neq$ 0 if and only if $g_{d}=s=s^{\prime}$. Moreover, $\chi \chi_{d}\left(\frac{u^{\prime}}{g}\right) \overline{\chi \chi_{d}}\left(\frac{u}{g}\right)=\chi \chi_{d}\left(u^{\prime} / s^{\prime}\right) \overline{\chi_{\chi}}(u / s)$. Finally, one shows that

$$
\begin{aligned}
\operatorname{Res}_{s=1} E(z, s) & =\pi \operatorname{Res}_{s=1}\left(\sum_{(c, 4 N)=1} \frac{\varphi(c)}{c^{2 s}}\right) \\
& =\frac{\pi}{4 N} \operatorname{Res}_{s=1}\left(\prod_{p \nmid 4 N} \frac{1-p^{-2 s}}{1-p^{1-2 s}}\right)=\frac{1}{2 \pi N} \prod_{p \mid N} \frac{p}{p+1} .
\end{aligned}
$$


Let $d$ satisfy $(D)$ and write $X=\frac{N}{f(d)^{2} d}$; let $X_{d}$ be the divisor of $X$ supported by $f(d)$ and write $X_{d}^{\prime}=X / X_{d}$. Let now $s$ satisfy the condition $\left(D_{d}\right)$, with $s$ supported by $f(d)$, i.e. $s^{2} \mid X_{d}$. Let finally $u$ and $v$ satisfy $u^{2}, v^{2} \mid X_{d}^{\prime}$. Then the preceding lemma gives

$$
\left\langle V_{d s^{2} u^{2}} \vartheta_{\chi \chi_{d}}(z), V_{d s^{2} v^{2}} \vartheta_{\chi \chi_{d}}(z)\right\rangle=C(N, d) \chi \chi_{d}(v) \overline{\chi \chi_{d}}(u) \frac{\operatorname{gcd}(u, v)}{s u v} .
$$

This leads to define

$$
\vartheta_{d, s, u}^{1}=C(N, d)^{-1 / 2} s^{\frac{1}{2}} u \chi \chi_{d}(u) V_{d s^{2} u^{2}} \vartheta_{\chi \chi_{d}} .
$$

These functions satisfy

$$
\left\langle\vartheta_{d, s, u}^{1}, \vartheta_{d, s, v}^{1}\right\rangle=\operatorname{gcd}(u, v), \quad \text { for any } u, v \text { so that } u^{2}, v^{2} \mid X_{d}^{\prime} .
$$

For a couple $(d, s)$ as above, we have to orthogonalise the set $\left\{\vartheta_{d, s, u}^{1}\right\}$, with $u^{2} \mid X_{d}^{\prime}$. This is achieved by the Möbius formula

$$
\sum_{j \mid u} \mu(j) f\left(\frac{u}{j}\right)=g(u) \Longleftrightarrow f(u)=\sum_{j \mid u} g(j) .
$$

Let us define the function

$$
\vartheta_{d, s, u}=\varphi(u)^{-1 / 2} \sum_{j \mid u} \mu(j) \vartheta_{d, s, \frac{u}{j}}^{1} .
$$

Lemma 1.5. Let d satisfy $(D)$. Let s satisfy $\left(D_{d}\right)$ and assume that $s$ is supported by $f(d)$. Let $u, v$ satisfy $\left(D_{d}\right)$ and assume $\operatorname{gcd}(u, f(d))=\operatorname{gcd}(v, f(d))=1$. Then

$$
\left\langle\vartheta_{d, s, u}, \vartheta_{d, s, v}\right\rangle= \begin{cases}1 & \text { if } u=v \\ 0 & \text { if } u \neq v\end{cases}
$$

Proof. By definition of $\vartheta_{d, s, u}$, the equality to be proven is equivalent to

$$
\varphi(u)^{-1 / 2} \sum_{j \mid u} \mu(j)\left\langle\vartheta_{d, s, \frac{u}{j}}^{1}, \vartheta_{d, s, v}\right\rangle= \begin{cases}1 & \text { if } u=v \\ 0 & \text { if } u \neq v\end{cases}
$$

that we rewrite as

$$
\sum_{j \mid u} \mu(j)\left\langle\vartheta_{d, s, \frac{u}{j}}^{1}, \vartheta_{d, s, v}\right\rangle= \begin{cases}\varphi(u)^{1 / 2} & \text { if } u=v \\ 0 & \text { if } u \neq v\end{cases}
$$

By choosing

$$
f(x)=\left\langle\vartheta_{d, s, x}^{1}, \vartheta_{d, s, v}\right\rangle \quad \text { and } \quad g(x)= \begin{cases}\varphi(x)^{1 / 2} & \text { if } x=v \\ 0 & \text { if } x \neq v\end{cases}
$$

in the Möbius formula (1.3.8), 1.3.10) is equivalent to

$$
\left\langle\vartheta_{d, s, u}^{1}, \vartheta_{d, s, v}\right\rangle=\sum_{j \mid u} \begin{cases}\varphi(j)^{1 / 2} & \text { si } j=v \\ 0 & \text { si } j \neq v\end{cases}
$$


By the definition (1.3.9) of $\vartheta_{d, s, v}$, and by evaluating the right hand side of (1.3.11), the equality of Lemma 1.5 is equivalent to

$$
\varphi(v)^{-1 / 2} \sum_{j \mid v} \mu(j)\left\langle\vartheta_{d, s, u}^{1}, \vartheta_{d, s, \frac{v}{j}}^{1}\right\rangle= \begin{cases}\varphi(v)^{1 / 2} & \text { si } v \mid u, \\ 0 & \text { si } v \nmid u,\end{cases}
$$

what we reformulate as

$$
\sum_{j \mid v} \mu(j)\left\langle\vartheta_{d, s, u}^{1}, \vartheta_{d, s, \frac{v}{j}}^{1}\right\rangle= \begin{cases}\varphi(v) & \text { si } v \mid u \\ 0 & \text { si } v \nmid u\end{cases}
$$

We apply the Möbius formula with

$$
f(x)=\left\langle\vartheta_{d, s, u}^{1}, \vartheta_{d, s, x}^{1}\right\rangle \quad \text { and } \quad g(x)= \begin{cases}\varphi(x) & \text { if } x \mid u, \\ 0 & \text { if } x \nmid u .\end{cases}
$$

Lemma 1.5 is then proved if and only if

$$
\left\langle\vartheta_{d, s, u}^{1}, \vartheta_{d, s, v}^{1}\right\rangle=\sum_{j \mid v} \begin{cases}\varphi(j) & \text { if } j \mid u \\ 0, & \text { si } j \nmid u\end{cases}
$$

The right hand side is equal to

$$
\sum_{j \mid \operatorname{gcd}(u, v)} \varphi(j)=\operatorname{gcd}(u, v)
$$

therefore $(1.3 .12)$ is verified and the lemma is proved.

Theorem 1.5. Let $N \in \mathbb{N}$. Let $\chi$ be a primitive Dirichlet character modulo $f$. Denote by $f(d)$ the conductor of the primitive Dirichlet character associated to the product of the characters $\chi$ and $\chi_{d}$. Define the following constant:

$$
c(N, d, s, u)=\left(\frac{2 \sqrt{d}}{\pi N} \prod_{p \mid N} \frac{p}{p+1} \prod_{p \mid f(d)} \frac{p}{p-1}\right)^{1 / 2} s^{\frac{1}{2}} \varphi(u)^{-1 / 2} .
$$

Then an orthonormal basis of $\operatorname{Mod}\left(4 N, \chi, \frac{1}{2}\right)$ is given by the set of functions $\vartheta_{d, s, u}(z)$ defined in (1.3.9, where the parameters $d, s, u$ satisfy

$$
\begin{aligned}
& \text { (i) } d \text { square-free, } \quad f(d)^{2} d \mid N, \\
& \text { (ii) } s^{2} \mid \frac{N}{f(d)^{2} d}, \quad \operatorname{supp}(s) \subseteq \operatorname{supp}(f(d)), \\
& \text { (iii) } u^{2} \mid \frac{N}{f(d)^{2} d}, \quad \operatorname{gcd}(u, f(d))=1
\end{aligned}
$$

Their Fourier expansions at infinity are given by

$$
\vartheta_{d, s, u}(z)=c(N, d, s, u) \sum_{m \in \mathbb{Z}} a_{d, s, u}(I d, m) e(m z)
$$


with Fourier coefficients

$$
a_{d, s, u}(I d, m)= \begin{cases}0 & \text { if } m \notin d s^{2} \mathbb{Z}^{2}, \\ \chi_{t}\left(m^{\prime}\right) \sum_{j \mid\left(u, m^{\prime}\right)} \mu\left(\frac{u}{j}\right) j & \text { if } m=d s^{2} m^{\prime 2} .\end{cases}
$$

Proof. As before, write $X=\frac{N}{f(d)^{2} d}$ and decompose $X=X_{d} X_{d}^{\prime}$, with $X_{d}$ supported by $d$. It remains to study the Fourier expansion of $\vartheta_{d, s, u}$. By (1.3.6) and (1.3.9), on a

$$
\begin{gathered}
\vartheta_{d, s, u}=C(N, d)^{-1 / 2} s^{\frac{1}{2}} \varphi(u)^{-1 / 2} \sum_{j \mid u} \mu(j) \frac{u}{j} \chi \chi_{d}\left(\frac{u}{j}\right) V_{d s^{2}(u / j)^{2}} \vartheta_{\chi \chi_{d}} \\
=c(N, d, s, u) \sum_{j \mid u} \mu\left(\frac{u}{j}\right) j \chi \chi_{d}(j) V_{d s^{2} j^{2}} \vartheta_{\chi \chi_{d}} .
\end{gathered}
$$

By the definition of $\vartheta_{\chi \chi_{d}}$, we obtain

$$
\begin{aligned}
\vartheta_{d, s, u}(z) & =c(d, s, u) \sum_{j \mid u} \mu\left(\frac{u}{j}\right) j \chi \chi_{d}(j) \sum_{n} \chi \chi_{d}(n) e\left(n^{2} d s^{2} j^{2} z\right) \\
& =c(N, d, s, u) \sum_{n} \chi \chi_{d}(n) \sum_{j \mid u} \mu\left(\frac{u}{j}\right) j \chi \chi_{d}(j) e\left(n^{2} d s^{2} j^{2} z\right) \\
& =c(N, d, s, u) \sum_{m} a_{m} e\left(m^{2} d s^{2} z\right),
\end{aligned}
$$

where

$$
a_{m}=\sum_{j \mid(u, m)} \chi \chi_{d}(m / j) \mu\left(\frac{u}{j}\right) j \chi \chi_{d}(j) .
$$

Since $u^{2} \mid X_{d}^{\prime}$, then $\operatorname{gcd}(u, f(d))=1$, and therefore, for any $j \mid u$, we have $\chi \chi_{d}(m / j)=\chi \chi_{d}(m) \overline{\chi \chi}(j)$. Thus

$$
a_{m}=\chi \chi_{d}(m) \sum_{j \mid(u, m)} \mu\left(\frac{u}{j}\right) j .
$$

Corollary 1.2. Let $D, f$ be odd positive integers, $\operatorname{gcd}(D, f)=1$. Let $\chi$ be a primitive character of conductor $f$. The space $\operatorname{Mod}\left(\Gamma_{0}(4 D f), \kappa \chi, \frac{1}{2}\right)$ is non-empty if and only if $f \equiv 1(\bmod 4)$ and $\chi=J_{f}=\chi_{f}$, in which case an orthonormal basis of it is given by the $\vartheta_{t f, s, u}(z)$, for $t^{3} \mid D$, $t \equiv 1(\bmod 4), s^{2} \mid \frac{D}{t^{3}}, s$ supported by $t, u^{2} \mid \frac{D}{t^{3}}, \operatorname{gcd}(u, t)=1$. For such parameter $t$, we have $\chi_{t f}=J_{t}=\chi_{t}$.

Proof. Recall that from Corollary 1.1 , we know that $d \equiv 0(\bmod f)$, for any $d$ satisfying $(D)$. Writing $d=f t$ and translating the conditions of Theorem 1.5 we get the result as stated. 


\subsection{Determination of the constant}

We are now in a position to determine explicitly both sides of the formula of Theorem 1.3 and then to prove Theorem 1.1. We assume that $D, f$ are odd, for simplicity. We choose the group $\Gamma_{0}(4 D f)$, for which the results of the previous section with $N=D f$ will be used. We still work with weight $k=1 / 2$.

\subsubsection{Choice of the cusps and of the level}

We choose the two cusps to be $\sigma^{-1}(\infty)$ and $\tau^{-1}(\infty)$ with $\tau^{-1}=I d$ and

$$
\sigma^{-1}=\left(\begin{array}{cc}
\alpha & \beta \\
D & 4 f
\end{array}\right)
$$

with $\alpha>0, \alpha$ even and $\beta$ odd. One verifies that $\Lambda_{\sigma}=4 f \mathbb{Z}, \Lambda_{\tau}=\mathbb{Z}$ and $\varkappa_{\sigma}=\varkappa_{\tau}=0$.

Proposition 1.7. The geometric Kloosterman sum appearing in the left hand side of the formula of Theorem 1.3 is given by

$$
K_{\sigma, \tau}(m, n, c)=\left\{\begin{array}{lll}
0 & \text { if } c \neq \equiv 0 \quad(\bmod D) \text { or if } c \text { is even } \\
\overline{\varepsilon_{D}}\left(\frac{c}{f}\right)_{2} \overline{\varepsilon_{c}} K_{2}(m, n, c) & \text { if } c \equiv 0 \quad(\bmod D) \text { and if } c \text { is odd }
\end{array}\right.
$$

We remark that for an odd integer $c$,

$$
\overline{\varepsilon_{c}} K_{2}(m, n, c) \in \mathbb{R} .
$$

Proof. Let $c>0$ be a fixed integer. By definition,

$$
K_{\sigma, \tau}(m, n, c)=\sum_{\substack{g \in G_{\sigma} \backslash G / G_{\tau} \\\left(\sigma g \tau^{-1}\right)= \pm c}} \overline{\psi(g) \sigma_{k}(\sigma, g) \sigma_{k}\left(\sigma g, \tau^{-1}\right)} e\left(\frac{\left(m-\frac{\varkappa_{\sigma}}{q_{\sigma}}\right) a\left(\sigma g \tau^{-1}\right)}{c}\right) e\left(\frac{\left(n-\frac{\varkappa_{\tau}}{q_{\tau}}\right) d\left(\sigma g \tau^{-1}\right)}{c}\right) .
$$

We replace $\sigma g \tau^{-1}$ by the matrix $\left(\begin{array}{ll}a & b \\ c & d\end{array}\right)$; this gives

$$
\begin{gathered}
K_{\sigma, \tau}(m, n, c)= \\
\sum_{\sigma^{-1}\left(\begin{array}{ll}
a & b \\
c & d
\end{array}\right) \tau \in G_{\sigma} \backslash G / G_{\tau}} \overline{\kappa \chi}\left(\sigma^{-1}\left(\begin{array}{ll}
a & b \\
c & d
\end{array}\right) \tau\right) \overline{\sigma_{k}}\left(\sigma, \sigma^{-1}\left(\begin{array}{ll}
a & b \\
c & d
\end{array}\right) \tau\right) \overline{\sigma_{k}}\left(\sigma \sigma^{-1}\left(\begin{array}{ll}
a & b \\
c & d
\end{array}\right) \tau, \tau^{-1}\right) \\
\times e\left(\frac{\left(m-\frac{\varkappa_{\sigma}}{q_{\sigma}}\right) a}{c}\right) e\left(\frac{\left(n-\frac{\varkappa_{\tau}}{q_{\tau}}\right) d}{c}\right) .
\end{gathered}
$$

If $g$ is replaced by $g_{\sigma} g g_{\tau}$, with $g_{\sigma}=\sigma^{-1} T^{\lambda_{\sigma}} \in G_{\sigma}$ and $g_{\tau}=\tau^{-1} T^{\lambda_{\tau}} \tau \in G_{\tau}$, then $\left(\begin{array}{ll}a & b \\ c & d\end{array}\right)=$ $\sigma g \tau^{-1}$ is replaced by $\sigma\left(\sigma^{-1} T^{\lambda_{\sigma}} \sigma\right) g\left(\tau^{-1} T^{\lambda_{\tau}} \tau\right) \tau^{1}=T^{\lambda_{\sigma}}\left(\begin{array}{ll}a & b \\ c & d\end{array}\right) T^{\lambda_{\tau}}$. Thus $a$ is defined modulo $\Lambda_{\sigma} c$ and $d$ modulo $\Lambda_{\tau} c$. It is easy to see that $\sigma_{k}\left(\left(\begin{array}{ll}a & b \\ c & d\end{array}\right), I d\right)=1$, thus 


$$
K(m, n, c)=\sum_{\substack{a(4 f c), d(c) \\
\sigma^{-1}\left(\begin{array}{ll}
a b \\
c & d
\end{array}\right) \tau \in \Gamma_{0}(4 D f)}} \overline{\kappa \chi}\left(\sigma^{-1}\left(\begin{array}{ll}
a & b \\
c & d
\end{array}\right) \tau\right) \overline{\sigma_{k}}\left(\sigma, \sigma^{-1}\left(\begin{array}{ll}
a & b \\
c & d
\end{array}\right) \tau\right) e\left(\frac{m a+n d}{c}\right)
$$

The condition

$$
\sigma^{-1}\left(\begin{array}{ll}
a & b \\
c & d
\end{array}\right) \in \Gamma_{0}(4 D f)
$$

means $a D+4 c f \equiv 0(\bmod 4 D f)$, thus $a \equiv 0(\bmod 4 f)$ (which implies $c$ odd) and $c \equiv 0$ $(\bmod D)$. Remark that $\sigma^{-1}\left(\begin{array}{ll}a & b \\ c & d\end{array}\right) \in \Gamma_{0}(4 f D)$, thus $\sigma^{-1} S(-S)\left(\begin{array}{ll}a & b \\ c & d\end{array}\right) \in \Gamma_{0}(4 f)$; since $\sigma^{-1} S \in \Gamma_{0}(4 f)$, so does $-S\left(\begin{array}{ll}a & b \\ c & d\end{array}\right)$. Finally, as $\kappa \chi$ is a multiplicative system, we have

$$
\begin{aligned}
& \overline{\kappa \chi_{f}}\left(\sigma^{-1}\left(\begin{array}{ll}
a & b \\
c & d
\end{array}\right)\right) \overline{\sigma_{k}}\left(\sigma, \sigma^{-1}\left(\begin{array}{ll}
a & b \\
c & d
\end{array}\right)\right)=\overline{\kappa \chi_{f}}\left(\sigma^{-1} S(-S)\left(\begin{array}{ll}
a & b \\
c & d
\end{array}\right)\right) \overline{\sigma_{k}}\left(\sigma, \sigma^{-1} S(-S)\left(\begin{array}{ll}
a & b \\
c & d
\end{array}\right)\right) \\
& =\overline{\kappa \chi_{f}}\left(\sigma^{-1} S\right) \overline{\kappa \chi_{f}}\left(-S\left(\begin{array}{ll}
a & b \\
c & d
\end{array}\right)\right) \overline{\sigma_{k}}\left(\sigma^{-1} S,-S\left(\begin{array}{ll}
a & b \\
c & d
\end{array}\right)\right) \overline{\sigma_{k}}\left(\sigma, \sigma^{-1} S(-S)\left(\begin{array}{ll}
a & b \\
c & d
\end{array}\right)\right) \\
& =\overline{\kappa \chi_{f}}\left(\sigma^{-1} S\right) \overline{\kappa \chi_{f}}\left(-S\left(\begin{array}{ll}
a & b \\
c & d
\end{array}\right)\right) \overline{\sigma_{k}}\left(\sigma, \sigma^{-1} S\right) \overline{\sigma_{k}}\left(S,-S\left(\begin{array}{ll}
a & b \\
c & d
\end{array}\right)\right) .
\end{aligned}
$$

Assume now that $a>0$. Then $\overline{\sigma_{k}}\left(\sigma, \sigma^{-1} S\right) \overline{\sigma_{k}}\left(S,-S\left(\begin{array}{ll}a & b \\ c & d\end{array}\right)\right)=1$. The choice $c>0$ and $a, d>0$ gives $b=\frac{a d-1}{c}>0$, and by the formula 1.3.5),

$$
\begin{aligned}
\kappa\left(-S\left(\begin{array}{ll}
a & b \\
c & d
\end{array}\right)\right) & =\kappa\left(\left(\begin{array}{cc}
c & d \\
-a & -b
\end{array}\right)\right):=\kappa\left(\left(\begin{array}{cc}
-c & -d \\
a & b
\end{array}\right)\right) \\
& =\left(\frac{-d}{b}\right)_{2} \varepsilon_{b} i=\left(\frac{-1}{b}\right)_{2}\left(\frac{d}{b}\right)_{2} \varepsilon_{b} i
\end{aligned}
$$

Since $a d-b c=1, b c \equiv-1(\bmod 4)$, and thus $\varepsilon_{b}\left(\frac{-1}{b}\right)_{2} i=\varepsilon_{c}$. It follows

$$
\kappa\left(-S\left(\begin{array}{ll}
a & b \\
c & d
\end{array}\right)\right)=\left(\frac{d}{b}\right)_{2} \varepsilon_{c}=\left(\frac{a}{b}\right)_{2} \varepsilon_{c}
$$

Since $a>0, a \equiv 0(\bmod 4)$ and $b c \equiv-1(\bmod a)$, it holds $\left(\frac{a}{b c}\right)_{2}=\left(\frac{a}{-1}\right)_{2}=1$, thus $\left(\frac{a}{b}\right)_{2}=\left(\frac{a}{c}\right)_{2}$. Finally, we have the following:

$$
\begin{gathered}
\kappa\left(\sigma^{-1} S\right)=\kappa\left(\left(\begin{array}{cc}
\beta & -\alpha \\
4 f & -D
\end{array}\right)\right):=\kappa\left(\left(\begin{array}{cc}
-\beta & \alpha \\
-4 f & D
\end{array}\right)\right)=\left(\frac{\alpha}{D}\right)_{2} \varepsilon_{D} \\
\chi\left(\sigma^{-1} S\right)=\chi(-D), \quad \chi\left(-S\left(\begin{array}{ll}
a & b \\
c & d
\end{array}\right)\right)=\chi(-b)
\end{gathered}
$$

Thus, if $c \equiv 0(\bmod D)$, 


$$
K_{\sigma, \tau}(m, n, c)=\sum_{\substack{a(4 f c) \\ d(c) \\ a \equiv=1(c) \\ a=0(4 f)}} \overline{\left(\frac{\alpha}{D}\right)_{2} \varepsilon_{D}\left(\frac{a}{c}\right)_{2} \varepsilon_{c} \chi(-D) \chi(-b)} e\left(\frac{m a+n d}{c}\right) .
$$

$4 \alpha f \equiv 1(\bmod D), \chi$ is a character of conductor $f$, and $a \equiv 0(\bmod f)$. This can be written as

$$
\begin{aligned}
K_{\sigma, \tau}(m, n, c) & =\overline{\varepsilon_{D}}\left(\frac{f}{D}\right)_{2} \bar{\chi}(-D) \chi(c) \overline{\varepsilon_{c}}\left(\frac{4 f}{c}\right)_{2} \sum_{\substack{a(c) d(c) \\
4 f a d \equiv 1(c)}}\left(\frac{a}{c}\right)_{2} e\left(\frac{m 4 f a+n d}{c}\right) \\
& =\overline{\varepsilon_{D} \varepsilon_{c}}\left(\frac{f}{D}\right)_{2} \bar{\chi}(-D) \chi(c) \sum_{\substack{x(c) d(c) \\
x d \equiv 1(c)}}\left(\frac{x}{c}\right)_{2} e\left(\frac{m x+n d}{c}\right) .
\end{aligned}
$$

From Corollary 1.2 , we know that $\chi=\chi_{f}$, for some square-free $f, f \equiv 1(\bmod 4)$. This finishes the proof of Proposition 1.7 .

In order to make explicit the right side of the formula of Theorem 1.3 , we need the Fourier expansion of the theta functions $\vartheta_{t f, s, u}(z)$ defined in 1.3 .9$)$ at both, $\tau^{-1}(\infty)$ and $\sigma^{-1}(\infty)$. While the former is given by Theorem 1.5 , we give here the latter. The cusp $\sigma^{-1}(\infty)$ is of width $4 f$. Recall that

$$
\vartheta_{t f, s, u}(z)=c(D f, t f, s, u) \sum_{j \mid u} \mu\left(\frac{u}{j}\right) j \chi_{t}(j) \vartheta_{\chi_{t}}\left(t f s^{2} j^{2} z\right),
$$

so that the first step is to study $\vartheta_{\chi_{t}}\left(t f s^{2} j^{2} z\right)$ at $\sigma^{-1}(\infty)$. Recall that $t \equiv 1(\bmod 4)$. More generally, we replace $\chi_{t}$ by $\psi$ and $t f s^{2} j^{2}$ by $t$. Let $r$ be the conductor of $\psi$. The condition that $t^{3} f s^{2} j^{2}$ divides $D f$ becomes now $r^{2} t \mid D f$. Let $r=r_{D} r_{f}$ and $t=t_{D} t_{f}$. Let $\psi_{D}, \psi_{f}$ be the associated characters to $\psi$. (For us, $\psi_{D}=\chi_{t}$ and $\psi_{f}=1$; moreover, $r_{D}=\operatorname{cond}\left(\chi_{t}\right)=t, r_{f}=1$, $\left.t_{D}=t s^{2} j^{2}, t_{f}=f\right)$.

Proposition 1.8. Let $\psi$ be a character of conductor $r$. Let $t$ be such that $r^{2} t \mid D f$. Let $\psi_{D}, \psi_{f}$ be the associated characters to $\psi$. Let $l \in \mathbb{N}-\{0\}$. Then, the $l^{\text {th }}$ Fourier coefficient of $\left(\sigma^{-1}\right)^{\prime}(z)^{1 / 4} \theta_{\psi}\left(t \sigma^{-1}(z)\right)$ in the expansion

$$
\left(\sigma^{-1}\right)^{\prime}(z)^{1 / 4} \theta_{\psi}\left(t \sigma^{-1}(z)\right)=\sum_{l>0} b(l) e\left(\frac{l^{2} t_{D} z}{4 r_{f}^{2} t_{f}}\right)
$$

is given by

$$
e^{i \pi / 4} \frac{1}{r_{f} \sqrt{2 t_{f}}}\left(\frac{t_{f} \alpha}{D t_{D}}\right)_{2} \varepsilon_{D t_{D}}\left(\psi_{D}(l)+\psi_{f}(-1) \psi_{D}(-l)\right) \psi_{D}\left(2 f \overline{r_{f} t_{f}}\right) \psi_{f}\left(-D \overline{t_{D} l}\right) G_{\psi_{f}}(1)
$$

Proof. We shall use a more general theta function. Let $\varepsilon, \varepsilon^{\prime} \in \mathbb{R}$. Define

$$
\theta\left(\varepsilon, \varepsilon^{\prime}, z\right)=\sum_{n \in \mathbb{Z}} e\left(\frac{1}{2}\left(n+\frac{\varepsilon}{2}\right)^{2} z\right) e\left(\left(n+\frac{\varepsilon}{2}\right) \frac{\varepsilon^{\prime}}{2}\right) .
$$


Then if $\gamma=\left(\begin{array}{ll}a & b \\ c & d\end{array}\right) \in \mathrm{SL}_{2}(\mathbb{Z}), z \in \mathbb{H}$ et $\varepsilon, \varepsilon^{\prime} \in \mathbb{R}$ we have ([8], Theorem 1.11 p. 81):

$$
\theta\left(\varepsilon, \varepsilon^{\prime}, \gamma(z)\right)=c\left(\varepsilon, \varepsilon^{\prime}, \gamma\right)(c z+d)^{1 / 2} \theta\left(a \varepsilon+c \varepsilon^{\prime}-a c, b \varepsilon+d \varepsilon^{\prime}+b d, z\right),
$$

with a constant $c\left(\varepsilon, \varepsilon^{\prime}, \gamma\right)$ satisfying

$$
c\left(\varepsilon, \varepsilon^{\prime}, \gamma\right)=e\left(-\frac{\left(a \varepsilon+c \varepsilon^{\prime}\right) b d}{4}-\frac{\left(a b \varepsilon^{2}+c d \varepsilon^{\prime 2}+2 b c \varepsilon \varepsilon^{\prime}\right)}{8}\right) c(0,0, \gamma) .
$$

The particular case $\varepsilon^{\prime}=0$ gives

$$
\theta(\varepsilon, 0, \gamma(z))=c(\varepsilon, 0, \gamma)(c z+d)^{1 / 2} \theta(a \varepsilon-a c, b \varepsilon+b d, z),
$$

with

$$
c(\varepsilon, 0, \gamma)=e\left(-\frac{\varepsilon a b d}{4}-\frac{\left(a b \varepsilon^{2}\right)}{8}\right) c(0,0, \gamma)
$$

Assume that $\gamma \in \Lambda$; Then $a c$ and $b d$ are even, and we have

$$
\begin{aligned}
\theta(a \varepsilon-a c, b \varepsilon+b d, z) & =\sum_{n \in \mathbb{Z}} e\left(\frac{1}{2}\left(n+\frac{a \varepsilon-a c}{2}\right)^{2} z\right) e\left(\frac{1}{2}\left(n+\frac{a \varepsilon-a c}{2}\right)(b \varepsilon+b d)\right) \\
& =\sum_{n \in \mathbb{Z}} e\left(\frac{1}{2}\left(n+\frac{a \varepsilon}{2}\right)^{2} z\right) e\left(\frac{1}{2}\left(n+\frac{a \varepsilon}{2}\right)(b \varepsilon+b d)\right) \\
& =e\left(\frac{a \varepsilon}{4}(b \varepsilon+b d)\right) \sum_{n \in \mathbb{Z}} e\left(\frac{1}{2}\left(n+\frac{a \varepsilon}{2}\right)^{2} z\right) e\left(\frac{1}{2} n b \varepsilon\right)
\end{aligned}
$$

and introducing this in 1.4 .2 gives

$$
\theta(\varepsilon, 0, \gamma(z))=e\left(\frac{\left(a b \varepsilon^{2}\right)}{8}\right) c(0,0, \gamma)(c z+d)^{1 / 2} \sum_{n \in \mathbb{Z}} e\left(\frac{1}{2}\left(n+\frac{a \varepsilon}{2}\right)^{2} z\right) e\left(\frac{1}{2} n b \varepsilon\right)
$$

Notice (p.81 of [8]), the authors work with $\arg (z) \in[0,2 \pi[$. Thus the constant $K(0,0, \gamma)$ is defined, in the case $\gamma \in \Lambda$, as

$$
\theta(0,0, \gamma(z))=c(0,0, \gamma)(c z+d)^{1 / 2} \theta(0,0, z) .
$$

Since $\theta(0,0, z)=\theta(z)$, we have

$$
c(0,0, \gamma)(c z+d)^{1 / 2}=\kappa_{\theta}(\gamma) \gamma^{\prime}(z)^{-1 / 4}
$$

We finally have

$$
\theta(\varepsilon, 0, \gamma(z))=e\left(\frac{\left(a b \varepsilon^{2}\right)}{8}\right) \kappa_{\theta}(\gamma) \gamma^{\prime}(z)^{-1 / 4} \sum_{n \in \mathbb{Z}} e\left(\frac{1}{2}\left(n+\frac{a \varepsilon}{2}\right)^{2} z\right) e\left(\frac{1}{2} n b \varepsilon\right) .
$$

Before starting the evaluation of the terms $b(l)$, we merely remark that

$$
\tilde{\sigma}=\left(\begin{array}{cc}
2 r_{f}^{2} t_{f} \alpha & r_{D}^{2} t_{D} \beta \\
D / r_{D}^{2} t_{D} & 4 f / 2 r_{f}^{2} t_{f}
\end{array}\right)
$$


is an element of $\Lambda$ and satisfies

$$
2 r^{2} t \sigma^{-1}(z)=\tilde{\sigma}\left(z^{\prime}\right), \quad \text { with } z^{\prime}=\frac{r_{D}^{2} t_{D}}{2 r_{f}^{2} t_{f}} z .
$$

Now,

$$
\begin{aligned}
\vartheta_{\psi}\left(t \sigma^{-1}(z)\right) & =\sum_{n \in \mathbb{Z}} \psi(n) e\left(n^{2} t \sigma^{-1}(z)\right)=\sum_{h(r)} \psi(h) \sum_{n \in \mathbb{Z}} e\left(\left(n+\frac{h}{r}\right)^{2} r^{2} t \sigma^{-1}(z)\right) \\
& =\sum_{h(r)} \psi(h) \sum_{n \in \mathbb{Z}} e\left(\frac{1}{2}\left(n+\frac{h}{r}\right)^{2} \tilde{\sigma}\left(z^{\prime}\right)\right)=\sum_{h(r)} \psi(h) \theta\left(\frac{2 h}{r}, 0, \tilde{\sigma}\left(z^{\prime}\right)\right)
\end{aligned}
$$

and by the formula (1.4.4,

$$
\begin{aligned}
\vartheta_{\psi}\left(t \sigma^{-1}(z)\right) & =(\tilde{\sigma})^{\prime}\left(z^{\prime}\right)^{-1 / 4} \kappa_{\theta}(\tilde{\sigma}) \sum_{h(r)} \psi(h) \sum_{n \in \mathbb{Z}} e\left(\left(n+\frac{2 \alpha h r_{f} t_{f}}{r_{D}}\right)^{2} \frac{z^{\prime}}{2}\right) e\left(\frac{n \beta h r_{D} t_{D}}{r_{f}}\right) \\
& =(\tilde{\sigma})^{\prime}\left(z^{\prime}\right)^{-1 / 4} \kappa_{\theta}(\tilde{\sigma}) \sum_{h(r)} \psi(h) \sum_{n \in \mathbb{Z}} e\left(\left(n r_{D}+2 \alpha h r_{f} t_{f}\right)^{2} \frac{z^{\prime}}{2 r_{D}^{2}}\right) e\left(\frac{n \beta h r_{D} t_{D}}{r_{f}}\right) \\
& =(\tilde{\sigma})^{\prime}\left(z^{\prime}\right)^{-1 / 4} \kappa_{\theta}(\tilde{\sigma}) \sum_{l \geqslant 0} b(l) e\left(l^{2} \frac{z^{\prime}}{2 r_{D}^{2}}\right) .
\end{aligned}
$$

Assume now that $l \neq 0$, then

$$
\begin{aligned}
b(l) & =\sum_{h(r)} \sum_{\substack{k \in \mathbb{Z} \\
\left(2 \alpha h r_{f} f_{f}+k r_{D}\right)^{2}=l^{2}}} \psi(h) e\left(\frac{k \beta h r_{D} t_{D}}{r_{f}}\right) \\
& =\sum_{h(r)} \sum_{\substack{k \in \mathbb{Z} \\
2 \alpha h r_{f} f_{f}^{+k r_{D}=l}}} \psi(h) e\left(\frac{k \beta h r_{D} t_{D}}{r_{f}}\right)+\sum_{h(r)} \sum_{\substack{k \in \mathbb{Z} \\
2 \alpha h r_{f} t_{f}+k r_{D}=-l}} \psi(h) e\left(\frac{k \beta h r_{D} t_{D}}{r_{f}}\right) .
\end{aligned}
$$

$h$ has to satisfy $2 \alpha h r_{f} t_{f} \equiv \pm l\left(\bmod r_{D}\right)$, in which case there exists $k \in \mathbb{Z}$ satisfying the condition, and one can replace $k r_{D}$ by an expression in terms of $h$; thus

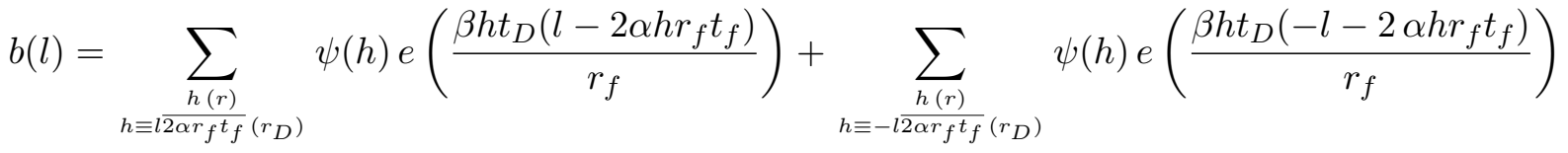

$$
\begin{aligned}
& =\sum_{h \equiv \frac{h(r)}{l 2 \alpha r_{f} t_{f}}\left(r_{D}\right)} \psi(h) e\left(\frac{\beta h t_{D} l}{r_{f}}\right)+\sum_{h \equiv-\frac{h(r)}{2 \alpha r_{f} t_{f}}\left(r_{D}\right)} \psi(h) e\left(-\frac{\beta h t_{D} l}{r_{f}}\right)
\end{aligned}
$$

We now decompose $\psi$ as $\psi=\psi_{D} \psi_{f}$, and we recall that the Gauss sum $G_{\chi}(m)=\sum_{k(\bmod r)} \chi(k) e^{2 i \pi m k / r}$, for $\chi$ primitive modulo $r, r>1$ satisfies $G_{\chi}(m)=\bar{\chi}(m) G_{\chi}(1)$. We obtain 


$$
\begin{aligned}
b(l) & =\psi_{D}\left(l \overline{2 \alpha r_{f} t_{f}}\right) \quad \sum_{h \equiv l \overline{h(r)}} \psi_{f}(h) e\left(\frac{\beta h t_{D} l}{r_{f}}\right)+\psi_{D}\left(-l \overline{2 \alpha r_{f} t_{f}}\right) \quad \sum_{\substack{h\left(r_{f} \\
h \equiv-l \overline{1 / 2 \alpha r_{f} t_{f}}\left(r_{D}\right)\right.}} \psi_{f}(h) e\left(-\frac{\beta h t_{D} l}{r_{f}}\right) \\
& =\psi_{D}\left(l \overline{2 \alpha r_{f} t_{f}}\right) \sum_{x\left(r_{f}\right)} \psi_{f}(x) e\left(\frac{\beta x t_{D} l}{r_{f}}\right)+\psi_{D}\left(-l \overline{2 \alpha r_{f} t_{f}}\right) \sum_{x\left(r_{f}\right)} \psi_{f}(x) e\left(-\frac{\beta x t_{D} l}{r_{f}}\right) \\
& =\left(\psi_{D}(l)+\psi_{f}(-1) \psi_{D}(-l)\right) \psi_{D}\left(\overline{2 \alpha r_{f} t_{f}}\right) \sum_{x\left(r_{f}\right)} \psi_{f}(x) e\left(\frac{\beta x t_{D} l}{r_{f}}\right) \\
& =\left(\psi_{D}(l)+\psi_{f}(-1) \psi_{D}(-l)\right) \psi_{D}\left(\overline{2 \alpha r_{f} t_{f}}\right) \overline{\psi_{f}}\left(\beta t_{D} l\right) G_{\psi_{f}}(1) \\
& =\left(\psi_{D}(l)+\psi_{f}(-1) \psi_{D}(-l)\right) \psi_{D}\left(2 f \overline{r_{f} t_{f}}\right) \psi_{f}\left(-D \overline{t_{D} l}\right) G_{\psi_{f}}(1) .
\end{aligned}
$$

We finally verify that

$$
(\tilde{\sigma})^{\prime}\left(z^{\prime}\right)^{-1 / 4}=\frac{\left(\sigma^{-1}\right)^{\prime}(z)^{-1 / 4}}{r_{f} \sqrt{2 t_{f}}}
$$

and

$$
\kappa_{\theta}(\tilde{\sigma})=e^{i \pi / 4}\left(\frac{t_{f} \alpha}{D t_{D}}\right)_{2} \varepsilon_{D t_{D}},
$$

and this finishes the proof of the Proposition 1.8 .

Proposition 1.9. Let $\sigma$ be as above. Let $f$ be an odd square free integer, $f>0, f \equiv 1(\bmod 4)$ and let $\chi=\chi_{f}$. Let $D$ be odd, and let $\vartheta_{t f, s, u}$ be an element of the base of $\operatorname{Mod}\left(\Gamma_{0}(4 D f), \chi \kappa, \frac{1}{2}\right)$. Then

$$
\sigma^{\prime}(z)^{-\frac{1}{4}} \vartheta_{t f, s, u}\left(\sigma^{-1}(z)\right)=\sum_{m \in(4 f)^{-1} \mathbb{Z}} a_{t f, s, u}(\sigma, m) e(m z)
$$

where

$$
a_{t f, s, u}(\sigma, m)= \begin{cases}0 & \text { if } m \notin \frac{t s^{2}}{4 f} \mathbb{Z}^{2}, \\ e^{i \pi / 4} \sqrt{\frac{2}{f}} \varepsilon_{D} \chi_{t}(2) c(D f, t f, s, u) \chi_{t}\left(m^{\prime}\right) \sum_{j \mid\left(u, m^{\prime}\right)} \mu\left(\frac{u}{j}\right) j & \text { if } m=m^{2} \frac{t s^{2}}{4 f} .\end{cases}
$$

Proof. From the definition of the functions $\vartheta_{t f, s, u}$ and the previous proposition, using the fact that $t \equiv 1(\bmod 4)$, we have

$$
\begin{aligned}
\sigma^{\prime}(z)^{-\frac{1}{4}} \vartheta_{t f, s, u}\left(\sigma^{-1}(z)\right) & =e^{i \pi / 4} \sqrt{\frac{2}{f}} \varepsilon_{D} \chi_{t}(2) c(D f, t f, s, u) \sum_{j \mid u} \mu\left(\frac{u}{j}\right) j \chi_{t}(j) \sum_{l>0} \chi_{t}(l) e\left(\frac{l^{2} t s^{2} j^{2} z}{4 f}\right) \\
& =e^{i \pi / 4} \sqrt{\frac{2}{f}} \varepsilon_{D} \chi_{t}(2) c(D f, t f, s, u) \sum_{n \in \mathbb{Z}} b(n) e\left(\frac{n t s^{2} z}{4 f}\right),
\end{aligned}
$$


where

$$
b(n)= \begin{cases}0 & \text { if } n \notin \mathbb{Z}^{2}, \\ \sum_{j \mid u} \mu\left(\frac{u}{j}\right) j \chi_{t}(j) \sum_{\substack{l>0 \\ l j=m}} \chi_{t}(l) & \text { if } n=m^{2}, m>0 .\end{cases}
$$

We can see that, for $n=m^{2}$,

$$
\begin{aligned}
b(n) & =\sum_{j \mid(u, m)} \mu\left(\frac{u}{j}\right) j \chi_{t}(j) \chi_{t}(m / j) \\
& =\chi_{t}(m) \sum_{j \mid(u, m)} \mu\left(\frac{u}{j}\right) j .
\end{aligned}
$$

Therefore we obtain

$$
\sigma^{\prime}(z)^{-\frac{1}{4}} \vartheta_{t f, s, u}\left(\sigma^{-1}(z)\right)=\sum_{m \in(4 f)^{-1} \mathbb{Z}} a_{t f, s, u}(\sigma, m) e(m z)
$$

where

$a_{t f, s, u}(\sigma, m)= \begin{cases}0 & \text { if } m \notin \frac{t s^{2}}{4 f} \mathbb{Z}^{2}, \\ e^{i \pi / 4} \sqrt{\frac{2}{f}} \varepsilon_{D} \chi_{t}(2) c(D f, t f, s, u) \chi_{t}\left(m^{\prime}\right) \sum_{j \mid\left(u, m^{\prime}\right)} \mu\left(\frac{u}{j}\right) j & \text { if } m=m^{\prime 2} \frac{t s^{2}}{4 f} .\end{cases}$

\subsubsection{Proof of Theorem 1.1}

First of all, let us rewrite both types of Fourier expansion needed here. Let $m, n \in \mathbb{Z}, m, n$ positive. One is, from Theorem 1.5 .

$$
a_{t, s, u}(\tau, n)= \begin{cases}0 & \text { if } n \notin t f s^{2} \mathbb{Z}^{2}, \\ c(D f, t f, s, u) \chi_{t}\left(n^{\prime}\right) \sum_{j \mid\left(u, n^{\prime}\right)} \mu\left(\frac{u}{j}\right) j & \text { if } n=t f s^{2} n^{\prime 2}\end{cases}
$$

The other is obtained by Proposition 1.9. As $m \in \mathbb{Z}$ and $m=m^{\prime 2} \frac{t s^{2}}{4 f}, m^{\prime}$ has to be a multiple of $2 f$, say $m^{\prime}=2 f m^{\prime \prime}$. Then, $m=m^{\prime \prime 2} f t s^{2}$ and

$$
a_{t f, s, u}(\sigma, m)=e^{i \pi / 4} \sqrt{\frac{2}{f}} \varepsilon_{D} c(D f, t f, s, u) \chi_{t}(f) \chi_{t}\left(m^{\prime \prime}\right) \sum_{j \mid\left(u, 2 f m^{\prime \prime}\right)} \mu\left(\frac{u}{j}\right) j .
$$

Since $\operatorname{gcd}(2 f, u)=1$, we reformulate this as 
$a_{t f, s, u}(\sigma, m)= \begin{cases}0 & \text { if } m \notin t f s^{2} \mathbb{Z}^{2}, \\ e^{i \pi / 4} \sqrt{\frac{2}{f}} \varepsilon_{D} c(D f, t f, s, u) \chi_{t}(f) \chi_{t}\left(m^{\prime}\right) \sum_{j \mid\left(u, m^{\prime}\right)} \mu\left(\frac{u}{j}\right) j & \text { if } m=t f s^{2} m^{\prime 2} .\end{cases}$

From (1.4.7) and (1.4.8), one sees that $\overline{a_{t f, s, u}}(\sigma, m) a_{t f, s, u}(\tau, n)$ is non-zero only if the square-free part of $m$ and $n$ are both equal to $t f$ for some integer $t \equiv 1(\bmod 4)$ such that $t^{3} \mid D$. Then, $\sqrt{\frac{m}{t f}}$ and $\sqrt{\frac{n}{t f}}$ has to be divisible by $s$, for some integer $s$ supported by $t$ and such that $s^{2} \mid \frac{d}{t^{3}}$. Therefore, we need

$$
m=t f s^{2} m^{\prime 2} \quad \text { and } \quad n=t f s^{2} n^{\prime 2} .
$$

As the factor $\chi_{t}\left(m^{\prime} n^{\prime}\right)$ appears, $m^{\prime}$ and $n^{\prime}$ have to be coprime with $t$. Thus, only the parameter $u$ is not determined by $m$ and $n$; it has to satisfy $u^{2} \mid \frac{D}{t^{3}}$ and to be coprime with $t$. Denote this set by $U$. Recall that $c(D f, t f, s, u)$ is defined in Theorem 1.5 . If $m$ and $n$ are as above, the right side of the formula of Theorem 1.3 is now completely determined; it is equal to

$$
\begin{gathered}
(1+i) \frac{8 f}{3 \pi} \sum_{u \in U} \overline{a_{t f, s, u}}(\sigma, m) a_{t f, s, u}(\tau, n) \\
=\frac{16 \sqrt{f}}{3 \pi} \overline{\varepsilon_{D}} \chi_{t}(f) \chi_{t}\left(m^{\prime} n^{\prime}\right) \sum_{u \in U} c(D f, t f, s, u)^{2} \sum_{j \mid\left(u, m^{\prime}\right)} \mu\left(\frac{u}{j}\right) j \sum_{j \mid\left(u, n^{\prime}\right)} \mu\left(\frac{u}{j}\right) j \\
=\frac{32}{3 \pi^{2}} \frac{\sqrt{t} s}{D} \prod_{p \mid D f} \frac{p}{p+1} \prod_{p \mid t} \frac{p}{p-1} \\
\times \overline{\varepsilon_{D}} \chi_{t}(f) \chi_{t}\left(m^{\prime} n^{\prime}\right) \sum_{u \in U} \varphi(u)^{-1} \sum_{j \mid\left(u, m^{\prime}\right)} \mu\left(\frac{u}{j}\right) j \sum_{j \mid\left(u, n^{\prime}\right)} \mu\left(\frac{u}{j}\right) j .
\end{gathered}
$$

We remark that $a(m) b(n)=a(n) b(m)$.

Let $X_{0}$ be the square-free part of $\frac{D}{t^{3}}$ and write $\frac{D}{t^{3}}=X_{0} X^{2}$; then $u \in \mathcal{U}$ if and only if $u \mid X$. If some $u \in \mathcal{U}$ divides $m$, then the corresponding sum over the divisors of $(u, m)$ is simply $\varphi(u)$. We set

$$
U^{\prime}=\left\{\prod_{p \mid X} p: \operatorname{ord}_{p}\left(\operatorname{gcd}\left(m^{\prime}, n^{\prime}, X\right)\right)<\operatorname{ord}_{p}(X)\right\}
$$

Introduce the notation $\min _{p}=\min \left(\operatorname{ord}_{p}\left(m^{\prime}\right), \operatorname{ord}_{p}\left(n^{\prime}\right)\right)$ for $p \mid U^{\prime}$. Define the subsets of $U^{\prime}$ as follows: 


$$
\begin{gathered}
U_{m}^{\prime}=\left\{\prod_{p \mid U^{\prime}}: \min _{p}=\operatorname{ord}_{p}\left(m^{\prime}\right)<\operatorname{ord}_{p}\left(n^{\prime}\right)\right\} \\
U_{n}^{\prime}=\left\{\prod_{p \mid U^{\prime}}: \min _{p}=\operatorname{ord}_{p}\left(n^{\prime}\right)<\operatorname{ord}_{p}\left(m^{\prime}\right)\right\} \\
U_{m, n}^{\prime}=\left\{\prod_{p \mid U^{\prime}}: \min _{p}=\operatorname{ord}_{p}\left(m^{\prime}\right)=\operatorname{ord}_{p}\left(n^{\prime}\right)\right\} .
\end{gathered}
$$

The presence of the Möbius function implies that any $u \in \mathcal{U}$ can be uniquely written as $u=$ $\prod_{p \mid u^{\prime}} p^{\min _{p}+1} u^{\prime \prime}$ with $u^{\prime} \mid U^{\prime}, \operatorname{gcd}\left(u^{\prime \prime}, u^{\prime}\right)=1$ and $u^{\prime \prime} \mid \operatorname{gcd}\left(X, m^{\prime}, n^{\prime}\right)$. We obtain, for a given $u=$ $u^{\prime} u^{\prime \prime}$, that

$$
\operatorname{gcd}\left(u, m^{\prime}\right)=u^{\prime \prime} \prod_{\substack{p \mid u^{\prime} \\ p \bigcup_{n}^{\prime}}} p^{\min _{p}} \prod_{\substack{p\left|u^{\prime} \\ p\right| U_{n}^{\prime}}} p^{\min _{p}+1}
$$

Therefore,

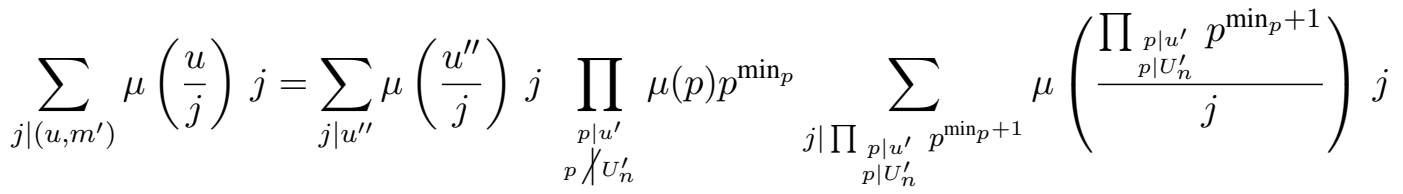

$$
\begin{aligned}
& =\varphi\left(u^{\prime \prime}\right) \prod_{\substack{p \mid u^{\prime} \\
p \nmid U_{n}^{\prime}}} \mu(p) p^{\min _{p}} \prod_{\substack{p\left|u^{\prime} \\
p\right| U_{n}^{\prime}}} p^{\min _{p}(p-1) .}
\end{aligned}
$$

The same holds for the second inner sum and we obtain

$$
\sum_{j \mid\left(u, m^{\prime}\right)} \mu\left(\frac{u}{j}\right) j \sum_{j \mid\left(u, n^{\prime}\right)} \mu\left(\frac{u}{j}\right) j=\varphi\left(u^{\prime \prime}\right)^{2} \prod_{p \mid u^{\prime}} p^{2 \min _{p}} \prod_{\substack{p \mid u^{\prime} \\ p \chi_{U_{m, n}^{\prime}}}} \mu(p)(p-1) .
$$

Finally, 


$$
\begin{aligned}
& \sum_{u \in U} \varphi(u)^{-1} \sum_{j \mid\left(u, m^{\prime}\right)} \mu\left(\frac{u}{j}\right) j \sum_{j \mid\left(u, n^{\prime}\right)} \mu\left(\frac{u}{j}\right) j \\
= & \sum_{u^{\prime} \mid U^{\prime}} \sum_{\substack{u^{\prime \prime} \mid\left(X, m^{\prime}, n^{\prime}\right) \\
\left(u^{\prime}, u^{\prime \prime}\right)=1}} \varphi\left(\prod_{p \mid u^{\prime}} p^{\min _{p}+1} u^{\prime \prime}\right)^{-1} \varphi\left(u^{\prime \prime}\right)^{2} \prod_{p \mid u^{\prime}} p^{2 \min _{p}} \prod_{\substack{p \mid u^{\prime} \\
p X_{m, n}^{\prime}}} \mu(p)(p-1) \\
= & \sum_{u^{\prime} \mid U^{\prime}} \varphi\left(u^{\prime}\right)^{-1} \prod_{p \mid u^{\prime}} p^{\min _{p}} \prod_{\substack{p \mid u^{\prime} \\
X_{U_{m, n}^{\prime}}}}(1-p) \sum_{\substack{u^{\prime \prime \prime} \mid(X, m, n) \\
\left(u^{\prime}, u^{\prime \prime}\right)=1}} \varphi\left(u^{\prime \prime}\right) \\
= & \operatorname{gcd}\left(X, m^{\prime}, n^{\prime}\right) \sum_{u_{1}^{\prime} \mid U_{m, n}^{\prime}} \varphi\left(u_{1}^{\prime}\right)^{-1} \sum_{\substack{u_{2}^{\prime} \mid \frac{U^{\prime}}{U_{m, n}^{\prime}} \\
\sum_{m, n}}} \mu\left(u_{2}^{\prime}\right) .
\end{aligned}
$$

This shows that $U_{m, n}^{\prime}$ has to be equal to $U^{\prime}$, and concludes the proof. 



\section{Cubic metaplectic forms}

In this chapter we present the theory of metaplectic forms, in the classical setting over $\mathrm{SL}_{2}(\mathbb{Z}[\omega])$ in Section 2.1, and in the representation theoretic context in Section2.3. Each point of view allow us to state interesting results that will be used in the next chapters.

More precisely, in Section 2.2, we present two general summation formulas for cubic Kloosterman sums; they relate sums of cubic Kloosterman sums with the spectral theory of the metaplectic forms developed in Section 2.1, we first obtain the so-called spectral summation formula in Theorem 2.4 and derive some consequences of it on the spectrum of the metaplectic group in Proposition 2.5. We finally obtain the so-called Kloosterman summation formula in Theorem 2.6 and derive some approximations formula for the inverse Bessel Transform in Proposition 2.6.

In Section 2.4, we give some relations between metaplectic forms and classical automorphic forms; they are consequences of the cubic Shimura correspondence, which relates genuine irreducible representations of the metaplectic group $\widetilde{\mathrm{GL}_{2}}\left(k_{\mathbb{A}}\right)$ with irreducible representations of $\mathrm{GL}_{2}\left(k_{\mathbb{A}}\right)$. Some special feature of the theory of metaplectic representations appear to be specific to the cubic metaplectic forms; it is an explanation of the fact that the determination of the explicit constant in the asymptotic behaviour of the twisted Kloosterman sums of order $j$

$$
K_{j}(m, n, c)=\sum_{x(c)}\left(\frac{x}{c}\right)_{j} e\left(\frac{m x+n x^{*}}{c}\right), \quad \text { for } j \geqslant 1
$$

can only be done in the quadratic case, i.e. for $j=2$, and in the cubic case, i.e. for $j=3$. The former was treated in Chapter 1 and the latter will be treated in Chapter 3. 


\subsection{Metaplectic forms on $\mathrm{SL}_{2}(\mathbb{Z}[\omega])$}

Let $k=\mathbb{Q}(\omega)$, where $\omega=e^{2 i \pi / 3}$. We define $\lambda=1-\omega$; it is the only prime in $R=\mathbb{Z}[\omega]$ which divides 3 . Any other prime in $R$ has a unique associated, i.e. a multiple by a unit of $R$, which is congruent to 1 modulo 3 . Such a prime will be called primary. Recall that the group of units in $R$ is $\mathcal{U}=\left\{ \pm 1, \pm \omega, \pm \omega^{2}\right\}$.

Let $\pi$ be a prime in $R$ coprime with 3 . Let $a$ be an integer of $R$, coprime with $\pi$. As the multiplicative group $(R / \pi R)^{\times}$is of order $\mathcal{N}(\pi)-1$, it holds

$$
a^{\mathcal{N}(\pi)-1} \equiv 1 \quad(\bmod \pi)
$$

As $\mathcal{N}(\pi) \equiv 1(\bmod 3)$, it exists a unique cubic root of 1 , say $\alpha$, such that

$$
a^{\frac{\mathcal{N}(\pi)-1}{3}} \equiv \alpha \quad(\bmod \pi)
$$

We define the cubic Legendre symbol by

$$
\left(\frac{a}{\pi}\right)_{3}=\alpha
$$

Let now $c$ be an integer of $R$ coprime with $3 a$; it has a decomposition $c=\eta \prod_{i} \pi_{i}^{e_{i}}$, with $\eta \in \mathcal{U}$. We define the Jacobi symbol by

$$
\left(\frac{a}{c}\right)_{3}=\prod_{i}\left(\frac{a}{\pi_{i}}\right)_{3}^{e_{i}} .
$$

The Jacobi symbol is multiplicative in $a$ and $c$, and satisfies

$$
\begin{array}{ll}
\left(\frac{a}{c}\right)_{3}=\left(\frac{b}{c}\right)_{3} & \text { if } a \equiv b \quad(\bmod c), \\
\overline{\left(\frac{a}{c}\right)_{3}}=\left(\frac{\bar{a}}{\bar{c}}\right)_{3} \quad \text { if } \operatorname{gcd}(a, c)=1 .
\end{array}
$$

Property 2.1.2 implies

$$
\left(\frac{a}{c}\right)_{3}=1 \quad \text { if } a, c \in \mathbb{Z}
$$

The main property of the Legendre-Jacobi symbol is the reciprocity law:

Theorem 2.1. Let $\pi_{1}, \pi_{2} \in \mathbb{Z}[\omega]$ be two primary primes. Then

$$
\left(\frac{\pi_{1}}{\pi_{2}}\right)_{3}=\left(\frac{\pi_{2}}{\pi_{1}}\right)_{3} .
$$

This theorem is completed by the complementary reciprocity laws:

Proposition 2.1. Let $\pi$ be a primary prime, $\pi=1+3(m+n \omega)$. Then 


$$
\begin{aligned}
\text { (i) }\left(\frac{\omega}{\pi}\right)_{3} & =\omega^{-m-n}, \\
\text { (ii) } & \left(\frac{\lambda}{\pi}\right)_{3}=\omega^{m}, \\
\text { (iii) } & \left(\frac{\pi}{\bar{\pi}}\right)_{3}=1 .
\end{aligned}
$$

Theorem[2.1 was proved by Eisenstein in [6] with the supplementary condition $\mathcal{N}\left(\pi_{1}\right) \neq \mathcal{N}\left(\pi_{2}\right)$. The remaining case, as well as he complementary reciprocity laws, were then proved by the same author in [7].

The three-dimensional hyperbolic space is usually represented as the half-space $\mathbb{H}=\mathbb{C} \times \mathbb{R}_{+}^{\times}$. We can embed it in the Hamiltonian quaternions by identifying $\sqrt{-1} \in \mathbb{C}$ with $\hat{i}$ and $w=(x+$ $i y, v) \in \mathbb{H}$ with $x+y \hat{i}+v \hat{k}$, where $1, \hat{i}, \hat{j}, \hat{k}$ are the standard unit quaternions. We shall use the same letter $\mathbb{H}$ as in Chapter 1 , but this should not cause confusion since the upper-half plane is used in this thesis only in Chapter 1 . The group $\mathrm{SL}_{2}(\mathbb{C})$ acts on $\mathbb{H}$ by

$$
\left(\begin{array}{ll}
a & b \\
c & d
\end{array}\right) w=(a w+b)(c w+d)^{-1}
$$

Explicitly, this action is given by

$$
\left(\begin{array}{ll}
a & b \\
c & d
\end{array}\right)(z, v)=\left(\frac{(a z+b) \overline{(c z+d)}+a \bar{c} v^{2}}{|c z+d|^{2}+|c|^{2} v^{2}}, \frac{v}{|c z+d|^{2}+|c|^{2} v^{2}}\right) .
$$

The Laplace-Beltrami operator is given by

$$
\Delta=v^{2}\left(\frac{\partial^{2}}{\partial x^{2}}+\frac{\partial^{2}}{\partial y^{2}}+\frac{\partial^{2}}{\partial v^{2}}\right)-v \frac{\partial}{\partial v}
$$

and the $\mathrm{SL}_{2}(\mathbb{C})$-invariant measure is $\mathrm{d} V(w)=v^{-3} \mathrm{~d} m(z) \mathrm{d} v$, where $\mathrm{d}(m(z)$ is the standard Lebesgue measure on $\mathbb{C}$.

Recall the Iwasawa decomposition of $G=\mathrm{SL}_{2}(\mathbb{C})$. We start by defining

$$
\begin{array}{ll}
H=\{h[u] u \in \mathbb{C}-\{0\} & \text { where } h[u]=\left(\begin{array}{cc}
u & 0 \\
0 & u^{-1}
\end{array}\right), \\
N=\{n[z], z \in \mathbb{C}\} & \text { where } n[z]=\left(\begin{array}{cc}
1 & z \\
0 & 1
\end{array}\right), \\
A=\{a[r], r>0\} & \text { where } a[r]=\left(\begin{array}{cc}
\sqrt{r} & 0 \\
0 & \sqrt{r}^{-1}
\end{array}\right), \\
K=S U(2) & \text { with elements } k(\alpha, \beta)=\left(\begin{array}{cc}
\alpha-\beta & \bar{\alpha}
\end{array}\right) .
\end{array}
$$

With the Euler angles $\phi, \theta, \psi \in \mathbb{R}$, we have 


$$
k[\alpha, \beta]=h\left[e^{i \phi / 2}\right] k[\cos \theta / 2, i \sin \theta / 2] h\left[e^{i \psi / 2}\right] .
$$

Then the Iwasawa decomposition states that

$$
G=N A K,
$$

which allows us to decompose any element $g$ of $G$ as $g=n a k=n[z] a[v] k[\alpha, \beta]$. The Haar measure on $N, A$ and $K$ are given by

$$
d n=d_{+} z, \quad d a=v^{-1} d v \quad \text { and } \quad d k=\left(16 \pi^{2}\right)^{-1} \sin \theta d \phi d \theta d \psi
$$

We define, for $u \in \mathbb{C}$, the character $\chi_{u}$ on $N$ by

$$
\chi_{u}(n)=e(u z), \quad \text { if } n=n[z] .
$$

Of first importance for us will be the subgroups of $\mathrm{SL}_{2}(\mathbb{Z}[\omega]$ defined by

$$
\begin{aligned}
\Gamma_{2} & =\left\{\gamma \in \mathrm{SL}_{2}(\mathbb{Z}[\omega]): \exists g \in \mathrm{SL}_{2}(\mathbb{Z}), \gamma \equiv g \quad(\bmod 3)\right\}, \\
\Gamma_{1} & =\left\{\gamma \in \mathrm{SL}_{2}(\mathbb{Z}[\omega]): \gamma \equiv 1 \quad(\bmod 3)\right\}, \\
\Gamma_{0}^{*}(d) & =\left\{\gamma \in \Gamma_{1}: c(\gamma) \equiv 0 \quad(\bmod d)\right\} .
\end{aligned}
$$

The Kubota symbol $\kappa$ can now be introduced. It is defined on $\Gamma_{1}$ by

$$
\kappa(\gamma)=\left\{\begin{array}{ll}
\left(\frac{c}{a}\right)_{3} & \text { if } c \neq 0 \\
1 & \text { if } c=0
\end{array}, \quad \text { where } \gamma=\left(\begin{array}{ll}
a & b \\
c & d
\end{array}\right) \in \Gamma_{1} .\right.
$$

This definition is then extended to $\Gamma_{2}$, the subgroup of $\mathrm{SL}_{2}(\mathbb{Z}[\omega])$ generated by $\Gamma_{1}$ and $\mathrm{SL}_{2}(\mathbb{Z})$, by defining $\kappa$ trivially on $\mathrm{SL}_{2}(\mathbb{Z})$. More precisely, for any $\gamma_{2} \in \Gamma_{2}$, there exists some $g \in \mathrm{SL}_{2}(\mathbb{Z})$ and $\gamma_{1} \in \Gamma_{1}$ such that $\gamma_{2}=g \gamma_{1}$, and we define

$$
\kappa\left(\gamma_{2}\right):=\kappa\left(\gamma_{1}\right)
$$

The starting point of the theory of metaplectic forms originates from the short and significant paper of Kubota ([22]), in which he proved that $\kappa$ is a group homomorphism on $\Gamma_{1}$. Actually, Kubota proved that $\kappa$ is a morphism on the subgroup of $\Gamma_{1}$ consisting in matrices congruent to 1 modulo 9, but this last condition can be dropped, and it can be proved that the definition of $\kappa$ on $\Gamma_{2}$ extend $\kappa$ to a group homomorphism from $\Gamma_{2}$ into the cubic roots of unity (see [32], p.127). A fundamental point in this theory is that the kernel of $\kappa$ does not contains a congruence subgroup. This is equally proved in [22], and leads to complications which do not usually appear in the theory of automorphic forms. We shall come back to this in Section 2.3.1.

Let $\Gamma$ be a subgroup of $\Gamma_{2}$ and let $\chi: \Gamma \longrightarrow \mathbb{C}$ be a group homomorphism. Inside the space of functions $f: \mathbb{H} \longrightarrow \mathbb{C}$ such that

$$
f(\gamma(w))=\kappa \chi(\gamma) f(w) \quad \forall \gamma \in \Gamma
$$

we distinguish two subspaces, the one formed by the square integrable function on $\Gamma \backslash \mathbb{H}$ with respect to $\mathrm{d} V(w)$, denoted by $\mathrm{L}^{2}(\Gamma \backslash \mathbb{H}, \kappa \chi)$ and the one of functions $f$ with $(\Delta+s(2-s)) f=0$ 
for some $s \in \mathbb{C}$, denoted by $\mathcal{M}(\Gamma \backslash \mathbb{H}, \kappa \chi, s)$.

In this section, we will deal with automorphic forms not only invariant by a subgroup of $\mathrm{SL}_{2}(\mathbb{Z}[\omega])$ but more generally by a subgroup of $G=\mathrm{SL}_{2}(\mathbb{C})$. As $G=N A K$ and $\mathbb{H} \cong N A$, this amounts to deal with general $K$-types (Section 2.1.1); these functions generalize the classical holomorphic functions on the upper half-plane, and are needed in order to achieve a complete summation formula for Kloosterman sums. Then, we shall present in Section 2.1.2 the spectral theory of $\mathrm{L}^{2}(\Gamma \backslash \mathbb{H}, \kappa \chi)$. Finally, we define the Poincare series in Section 2.1.3 and, as we did in Chapter 1, we shall make appear the cubic Kloosterman sums $K_{3}(m, n, c)$ as Fourier coefficients of these Poincaré series.

\subsection{1 $K$-types and metaplectic forms}

We need some facts about the Lie algebra of $G$ and $K$; we simply excerpt them from Section 3 of [4]. The real Lie algebra $\mathfrak{s l}(2)$ of $G$ is a six dimensional real vector space generated by $\left\{H_{1}, H_{2}, V_{1}, V_{2}, W_{1}, W_{2}\right\}$, where

$$
\begin{array}{rlrl}
H_{1} & =\frac{1}{2}\left(\begin{array}{cc}
1 & 0 \\
0 & -1
\end{array}\right), & V_{1}=\frac{1}{2}\left(\begin{array}{cc}
0 & 1 \\
1 & 0
\end{array}\right), & W_{1}=\frac{1}{2}\left(\begin{array}{cc}
0 & 1 \\
-1 & 0
\end{array}\right) \\
H_{2}=\frac{1}{2}\left(\begin{array}{cc}
i & 0 \\
0 & -i
\end{array}\right), & V_{2}=\frac{1}{2}\left(\begin{array}{cc}
0 & i \\
-i & 0
\end{array}\right), & W_{2}=\frac{1}{2}\left(\begin{array}{cc}
0 & i \\
i & 0
\end{array}\right) .
\end{array}
$$

The Casimir elements are defined by

$$
\Omega_{ \pm}=\frac{1}{8}\left(\left(H_{1} \mp i H_{2}\right)^{2}+\left(V_{1} \mp i W_{2}\right)^{2}-\left(W_{1} \mp i V_{2}\right)^{2}\right) .
$$

The center $\mathcal{Z}(\mathfrak{s l}(2))$ of the universal covering $\mathcal{U}(\mathfrak{s l}(2))$ of $\mathfrak{s l}_{2}$ is the polynomial ring $\mathbb{C}\left[\Omega_{+}, \Omega_{-}\right]$.

The real Lie algebra $\mathfrak{s u}(2)$ of $K$ is generated by $H_{2}, W_{1}$ and $W_{2}$; its complexification is $\mathfrak{s l}_{2}(\mathbb{C})$. The Casimir element is defined by

$$
\Omega_{\mathfrak{k}}=\frac{-1}{2}\left(H_{2}^{2}+W_{1}^{2}+W_{2}^{2}\right) .
$$

The center $\mathcal{Z}(\mathfrak{s u}(2))$ of the universal covering $\mathcal{U}(\mathfrak{s u}(2))$ of $\mathfrak{s l}_{2}(\mathbb{C})$ is the polynomial ring $\mathbb{C}\left[\Omega_{\mathfrak{k}}\right]$.

We shall use this to give results about the irreducible unitary representations of $K$, the goal being to study the Hilbert space $\mathrm{L}^{2}(K)$ of functions on $K$ which are square-integrable over $K$ with respect to the Haar measure $d k$. First recall that finite dimensional representations of $G$ are given by $\left(T_{n}, V_{n}\right)$, for $n \in \mathbb{N}$, where $V_{n}$ is the space of polynomial in one variable of degree at most $n$, and $T_{n}: G \longrightarrow \operatorname{Aut}\left(V_{n}\right)$ is a homomorphism given by

$$
T_{n}(g)(f(z))=(b z+d)^{n} f\left(\frac{a z+c}{b z+d}\right) .
$$

Write $n=2 l$ and define $\sigma_{l}$ as the restriction of $T_{2 l}$ to $K$. Then $l \in \frac{1}{2} \mathbb{N}$, and any element of $V_{2 l}$ is of the form $z^{l-q} ; q$ has to satisfy the conditions $|q| \leqslant l$ and $q \equiv l(\bmod 1)$, which ensures that $l-q \in \mathbb{N}$. The representation $\sigma_{l}$ is irreducible, and we can rewrite the action of $K$ on the element $z^{l-q}$ of $V_{2 l}$ as 


$$
\sigma_{l}(k)\left(z^{l-q}\right)=(\alpha z-\bar{\beta})^{l-q}(\beta z+\bar{\alpha})^{l+q} \in V_{2 l}, \quad \text { for } k=k(\alpha, \beta),
$$

and its expansion in $V_{2 l}$ is of the form

$$
\sigma_{l}(k)\left(z^{l-q}\right)=\sum_{|p| \leqslant l} \phi_{p, q}^{l}(k) z^{l-p},
$$

where the functions $\phi_{p, q}^{l}$, with $l \geqslant 0,|p| \leqslant l,|q| \leqslant l$ and $p \equiv q \equiv l(\bmod 1)$, are functions on $K$. Actually, the $\phi_{p, q}^{l}$ form an orthogonal basis of $L^{2}(K)$ and satisfy

$$
\begin{aligned}
H_{2} \phi_{p, q}^{l} & =-i q \phi_{p, q}^{l}, \quad \Omega_{\ell} \phi_{p, q}^{l}=-\frac{1}{2}\left(l^{2}+l\right) \phi_{p, q}^{l} \\
\left\|\phi_{p, q}^{l}\right\|_{K} & =\frac{1}{\sqrt{2 l+1}}\left(\begin{array}{c}
2 l \\
l-p
\end{array}\right)^{\frac{1}{2}}\left(\begin{array}{c}
2 l \\
l-q
\end{array}\right)^{\frac{-1}{2}} .
\end{aligned}
$$

Moreover, all irreducible unitary representations of $K$ are obtained in this way. From this discussion, we obtain the following decomposition of $\mathrm{L}^{2}(K)$ :

$$
\mathrm{L}^{2}(K)=\bigoplus_{\substack{l, q \\|q| \leq l \\ q \equiv l(1)}} \mathrm{L}^{2}(K, l, q), \quad \mathrm{L}^{2}(K, l, q)=\bigoplus_{\substack{|p| \leqslant l \\ p \equiv l(1)}} \mathbb{C} \phi_{p, q}^{l}
$$

We can see $\mathrm{L}^{2}(K, l, q)$ as the set of functions $f$ on $\mathrm{L}^{2}(K)$ satisfying

$$
\begin{aligned}
& (*) \quad \Omega_{\mathfrak{l}} f=-\left(l^{2}+1\right) / 2 f \\
& (* *) \quad H_{2} f=-i q f .
\end{aligned}
$$

More generally, a function $f$ of a space $V$ is said to be of $K$-type $(l, q)$ if $K$ acts on $V$ and if $f$ satisfies $(*)$ and $(* *)$. This concept correspond to the weight in the theory of modular forms on the upper half plane (see Chapter 1). The functions $\phi_{p, q}^{l}$ are examples of functions of type $(l, q)$. Another example is the function $\varphi_{l, q}(s, p): G \longrightarrow \mathbb{C}$, defined by

$$
\varphi_{l, q}(s, p)(g)=v^{1+s} \phi_{p, q}^{l}(k), \quad \text { if } g=n[z] a[v] k .
$$

The function $\varphi_{l, q}(s, p)$ satisfies

$$
\Omega_{ \pm} \varphi_{l, q}(s, p)=\frac{1}{8}\left((s-\mp p)^{2}-1\right) \varphi_{l, q}(s, p) .
$$

The spaces

$$
H(s, p)=\left\{\text { finite linear combinations of } \varphi_{l, q}(s, p)\right\}
$$

will be seen as models for irreducible subspaces of the space of square-integrable metaplectic forms, that we are now able to define.

We conclude this section by giving the definition of metaplectic forms, and showing which part the function $\varphi_{l, q}(s, p)$ plays in their Fourier expansions. 
Definition 2.1. Let $\Gamma$ be a discrete subgroup of $\Gamma_{2}$. Define by $\mathcal{A}_{l, q}(\Gamma \backslash G, \kappa,(s, p))$ the subspace of smooth functions $f$ on $G$ satisfying

$$
\left.\begin{array}{l}
f(\gamma g)=\kappa(\gamma) f(g) \\
\Omega_{ \pm} f=\frac{1}{8}\left((s \mp p)^{2}-1\right) f \\
f \text { is of type }(l, q) .
\end{array}\right\}
$$

An element $f$ of $\mathcal{A}_{l, q}(\Gamma \backslash G, \kappa,(s, p))$ is called a metaplectic form for $\Gamma$ of type $(l, q)$ and of spectral parameter $(s, p)$.

Remark 2.1. In particular, if $-I d \in \Gamma$ and $f \in \mathcal{A}_{l, q}(\Gamma \backslash G, \kappa,(s, p))$, we see from the definition that, on one side, $f(-g)=\chi(-I d) f(g)=f(g)$, and that, on the other side, $f(-g)=$ $\sigma_{l}(-I d) f(g)=e^{2 i q \pi} f(g)$. The only possible types $(l, q)$ are then the ones with $l, q, p \in \mathbb{Z}$. As we will see in Remark 2.2 $p$.61 an extra condition appears in the spectral sum formula if there exist spectral parameters $(s, p)$ with $p \in \frac{1}{2}+\mathbb{Z}$. This justifies that we consider the group $\Gamma_{d}=\left\langle\Gamma_{1} \cap \Gamma_{0}(d),-I d\right\rangle$ rather than $\Gamma_{0}^{*}(d)=\Gamma_{1} \cap \Gamma_{0}(d)$ considered in [31].

According to this remark, it is reasonable to define

$$
\Gamma_{1}^{-}=\left\{\gamma \in \mathrm{SL}_{2}(\mathbb{Z}[\omega]): \gamma \equiv \pm 1 \quad(\bmod 3)\right\}
$$

Lemma 2.1. Let $\Gamma$ be any discrete subgroup of $\Gamma_{1}^{-}$containing $-I d$, and let $\Gamma_{\sigma}$ be the stabilizator in $\Gamma$ of the cusp $\sigma^{-1}(\infty)$, where $\sigma \in \mathrm{SL}_{2}(\mathrm{R})$. Then there exists some lattice $\Lambda_{\sigma}$ in $R, \Lambda_{\sigma} \subseteq(3)$, such that

$$
\Gamma_{\sigma}=\sigma^{-1}\left(\begin{array}{cc}
1 & \Lambda_{\sigma} \\
0 & 1
\end{array}\right) \sigma \cup \sigma^{-1}\left(\begin{array}{cc}
-1 & \Lambda_{\sigma} \\
0 & -1
\end{array}\right) \sigma .
$$

Let $\Gamma$ be a subgroup of $\Gamma_{1}^{-}$. If a function $f$ satisfies $f(\gamma g)=\kappa(\gamma) f(g)$, for all $\gamma \in \Gamma$, then $f$ has a Fourier expansion

$$
f\left(\sigma^{-1} g\right)=\sum_{m \in \Lambda_{\hat{\sigma}}} F_{f}(\sigma, m)(g)
$$

with Fourier coefficients

$$
F_{f}(\sigma, m)(g)=\int_{\sigma \Gamma_{\sigma} \sigma^{-1} \backslash N} \overline{\chi_{m}}(n) f(n g) d n .
$$

Let $W_{l, q}(s, p, m)$ be the space

$W_{l, q}(s, p, m)=\left\{h \in C^{\infty}(G): h(n g)=\chi_{m}(n) h(g), h\right.$ is of $K$-type $(l, q)$ and of spectral parameter $\left.(s, p)\right\}$, with $\chi_{m}$ as in 2.1.4. If $m=0$, then $\operatorname{dim}\left(W_{l, q}(s, p, m)\right)=2$ and one has

$$
\begin{aligned}
W_{l, q}(s, p, 0) & =\mathbb{C} \varphi_{l, q}(s, p) \oplus \mathbb{C} \varphi_{l, q}(-s,-p), \text { if }(s, p) \neq(0,0) \\
W_{l, q}(, 0,0) & =\left.\mathbb{C} \varphi_{l, q}(0,0) \oplus \mathbb{C} \partial_{s} \varphi_{l, q}(s, p)\right|_{s=0} .
\end{aligned}
$$


If $f \in \mathcal{A}_{l, q}(\Gamma \backslash G, \kappa,(s, p))$, one sees that $F_{f}(\sigma, m) \in W_{l, q}(s, p, m)$. Actually, for any $m \neq$ $0, W_{l, q}(s, p, m)$ is of dimension 2 , and contains as one dimensional subspace, the subspace $W_{l, q}^{\mathrm{pol}}(s, p, m)$ of functions of polynomial growth. If a metaplectic form $f$ is of polynomial growth, i.e. $f(n a[v] k) \ll v^{\alpha}$, then its Fourier coefficients inherit this growth property, and are multiple of the generator of $W_{l, q}^{\mathrm{pol}}(s, p, m)$. This generator is known; see Section 5 of [4]. For it we need to define the operator $\mathcal{A}_{m}$, on the space of functions $f \in C^{\infty}(G)$ such that, for some $\sigma>0$,

$$
f(n a[v] k)=\mathcal{O}\left(v^{1+\sigma}\right), \quad \text { as } v \rightarrow 0 .
$$

It is defined by

$$
\mathcal{A}_{m} f(g)=\int_{N} \bar{\chi}_{m}(n) f(w n g) d n .
$$

Then, one proves that, for any spectral parameter $(s, p)$, if $m \neq 0, W_{l, q}^{\mathrm{pol}}(s, p, m)$ is generated by $\mathcal{A}_{m} \varphi_{l, q}(s, p)$.

Proposition 2.2. Let $f$ be a square-integrable metaplectic form of spectral parameter $(s, p)$. Let $\Gamma$ be a subgroup of $\Gamma_{1}^{-}$and let $\sigma^{-1}(\infty)$ be a cusp of $\Gamma$. Then,

$$
f\left(\sigma^{-1} g\right)=\sum_{m \in \Lambda_{\hat{\sigma}}} \rho_{f}(m) \mathcal{A}_{m} \varphi_{l, q}(s, p)(g) .
$$

The function $f$ is said to be a cuspform if $\rho_{f}(\sigma, 0)=0$ for all cusps $\sigma^{-1}(\infty)$ of $\Gamma$. The space $\mathrm{L}^{2, \text { cusp }}(\Gamma \backslash G, \kappa)$ generated by the cuspforms is a subspace of $\mathrm{L}^{2}(\Gamma \backslash G, \kappa)$.

\subsubsection{Eisenstein series, theta functions and the spectral decomposi- tion theorem}

Let $\Gamma \subset \Gamma_{1}^{-}$. A cusp $\sigma^{-1}(\infty)$ of $\Gamma$, with $\sigma^{-1} \in \mathrm{SL}_{2}(\mathbb{Z}[\omega])$, is called essential if $\left.\kappa\right|_{\Gamma_{\sigma}}=1$. The set of $\Gamma$-inequivalent essential cusps will be denoted by $\mathcal{C}(\Gamma)$. Eisenstein series are defined for essential cusps.

Definition 2.2. Let $\sigma^{-1}(\infty)$ be an essential cusp of $\Gamma$. Let $p, l, q \in \mathbb{Z},|p|,|q| \leqslant l$, and $p \equiv l \equiv q$ $(\bmod 1)$. For $\Re(s)>1$, the Eisenstein series $E_{\sigma}(s, p, l, q ; g)$ is

$$
E_{\sigma}(s, p, l, q ; g)=\sum_{\gamma \in \Gamma_{\sigma} \backslash \Gamma} \bar{\kappa}(\gamma) \varphi_{l, q}(s, p)(\sigma \gamma g) .
$$

It admits a Fourier expansion at any cusp of $\Gamma$, but for simplicity, we will only work with essential cusps. If $\tau^{-1}(\infty)$ is an essential cusp of $\Gamma$, then

$$
\begin{aligned}
& E_{\sigma}\left(s, p, l, q ; \tau^{-1} g\right)=\delta_{\sigma, \tau} \varphi_{l, q}(s, p)(g) \\
& +\frac{\pi(-1)^{p-|p|}}{\operatorname{Vol}\left(\Lambda_{\tau}\right)} \frac{\Gamma(l+1-s) \Gamma(|p|+s)}{\Gamma(l+1+s) \Gamma(|p|+1-s)} \psi_{\sigma, \tau}(s, 0, p) \varphi_{l, q}(-s,-p)(g) \\
& +\frac{1}{\operatorname{Vol}\left(\Lambda_{\tau}\right)} \sum_{0 \neq m \in \Lambda_{\hat{\tau}}} \psi_{\sigma, \tau}(s, m, p) \mathcal{A}_{m} \varphi_{l, q}(s, p)(g),
\end{aligned}
$$

whose Fourier coefficients $\psi_{\sigma, \tau}(s, m, p)$ are Dirichlet series formed by Gauss sums, i.e. 


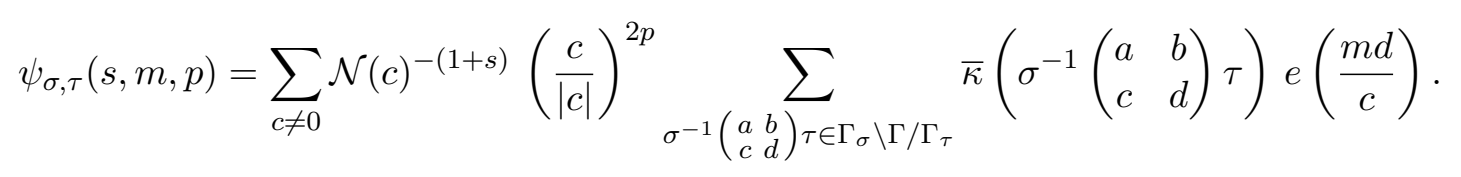

The properties needed for our applications are listed below. Consider $E_{\sigma}(s, p, l, q ; g)$ as a function of the variable $s$. Then

(i) $E_{\sigma}(s, p, l, q ; g)$ possesses a meromorphic continuation to $\mathbb{C}$ and a functional equation relating $E_{\sigma}(1+s, p, l, q ; g)$ and $E_{\sigma}(1-s, p, l, q ; g)$,

(ii) $E_{\sigma}(s, p, l, q ; g)$ is holomorphic if $p \neq 0$,

(iii) $E_{\sigma}(s, p, l, q ; g)$ has poles at $s=\frac{-1}{3}, \frac{1}{3}$ if $p=0$,

The last property is new, when compared to the classical theory of automorphic forms whose automorphic factor is a Dirichlet character. Actually, taking the residue of Eisenstein series give some square-integrable non cuspidal automorphic forms. They are eigenfunction of the Laplacian with eigenvalue $1-s^{2}$. This is the minimal eigenvalue of the Laplacian, and in our case it is $1-s^{2}=8 / 9$.

Definition 2.3. In the half-plane $\Re(s) \geqslant 0$, the theta function associated to the essential cusp $\sigma^{-1}(\infty)$ is defined as

$$
\theta_{\sigma}((l, q), g)=\operatorname{Res}_{s=1 / 3}\left(E_{\sigma}(s, 0, l, q ; g)\right) .
$$

It is a square integrable non cuspidal metaplectic forms of spectral parameter $\left(\frac{1}{3}, 0\right)$ and of $K$-type $(l, q)$.

From the Fourier expansion of $E_{\sigma}(s, p, l, q ; g)$, one gets

$$
\begin{aligned}
\theta_{\sigma}\left((l, q), \tau^{-1} g\right)= & \frac{\pi}{\operatorname{Vol}\left(\Lambda_{\tau}\right)} \frac{\Gamma(l+2 / 3) \Gamma(1 / 3)}{\Gamma(l+4 / 3) \Gamma(2 / 3)} \rho_{\theta_{\sigma}}(\tau, 0) \varphi_{l, q}(-1 / 3,0)(g) \\
& +\frac{1}{\operatorname{Vol}\left(\Lambda_{\tau}\right)} \sum_{0 \neq m \in \Lambda_{\hat{\tau}}} \rho_{\theta_{\sigma}}(\tau, m) \mathcal{A}_{m} \varphi_{l, q}(1 / 3,0)(g),
\end{aligned}
$$

where

$$
\rho_{\theta_{\sigma}}(\tau, m)=\operatorname{Res}_{s=1 / 3}\left(\psi_{\sigma, \tau}(s, m, 0)\right) .
$$

We refer to Section 3.1.2 of Chapter 3 for more details on the function $\rho$.

Let $\mathrm{L}^{2, \text { res }}(\Gamma \backslash G, \kappa)$ be the space generated by the theta series $\theta_{\sigma}((l, q), g)$, for $l \in \mathbb{N}, q \in \mathbb{Z}$, $|q| \leqslant l$ and $\sigma^{-1}(\infty)$ an essential cusp of $\Gamma$.

Theorem 2.2. Let $\mathrm{L}^{2, \text { disc }}(\Gamma \backslash G, \kappa)$ be the direct sum of the invariant irreducible subspaces of $\mathrm{L}^{2}(\Gamma \backslash G, \kappa)$. Then $\mathrm{L}^{2, \text { disc }}(\Gamma \backslash G, \kappa)$ is the direct sum of $\mathrm{L}^{2, \text { res }}(\Gamma \backslash G, \kappa)$ and $\mathrm{L}^{2 \text {, cusp }}(\Gamma \backslash G, \kappa)$, and we have

$$
\mathrm{L}^{2}(\Gamma \backslash G, \kappa)=\mathrm{L}^{2, \text { res }}(\Gamma \backslash G, \kappa) \oplus \mathrm{L}^{2, \text { cusp }}(\Gamma \backslash G, \kappa) \oplus \mathrm{L}^{2, \text { cont }}(\Gamma \backslash G, \kappa) .
$$




\subsubsection{Poincaré series}

Let us define $C^{\infty}(N \backslash G, m)$ as the set of smooth functions on $G$ such that $f(n g)=\chi_{m}(n) f(g)$ for any $n \in N$. This kind of functions will be used as generating function for the Poincaré series. All results of this section are classical, and are proved in Section 2 for the case $\mathrm{SL}_{2}(\mathbb{R})$. Therefore we refer to the literature for the proofs.

The following two growth conditions for a function $f$ on $G$ will be useful in the sequel:

$$
\text { (C) } \begin{cases}f(n a k) \ll v^{1+\sigma_{0}}, & \text { as } v \text { tends to } 0, \text { for some } \sigma_{0}>0 \\ f(n a k) \ll v^{1-\sigma_{\infty}}, & \text { as } v \text { tends to } \infty, \text { for some } \sigma_{\infty}>0\end{cases}
$$

Poincaré series can be defined for any cusp, essential or not, but for simplicity, we shall work only with essential cusp.

Definition 2.4. Let $\sigma \in \mathrm{SL}_{2}(R)$ such that $\sigma^{-1}(\infty)$ is an essential cusp of $\Gamma$. Let $m \in \Lambda_{\sigma}^{\wedge}-\{0\}$ and $f \in C^{\infty}(N \backslash G, m)$. The Poincaré series generated by $f$ is

$$
P_{f}(\sigma, g)=\sum_{\gamma \in \Gamma_{\sigma} \backslash \Gamma} \bar{\kappa}(\gamma) f(\sigma \gamma g) .
$$

For example, we can take for $f(g)$ the function

$$
f_{m}(g)=f_{m}(n[z] a[v] k)=\chi_{m}(n) \exp (-4 \pi|m| v) v^{s} .
$$

In this case, we find the Poincare series used in [31]; it is defined for $\Re(s)>2$ and decreases exponentially at 0 .

Definition 2.5. Let $\sigma^{-1}, \tau^{-1} \in \mathrm{SL}_{2}(\mathbb{Z}[\omega])$ and let $m \in \Lambda_{\sigma}^{\wedge}$ and $n \in \Lambda_{\tau}^{\wedge}$. Then we define the Kloosterman sum at $c \in \mathbb{Z}[\omega]-\{0\}$ by

$$
K_{\sigma, \tau}(m, n, c)=\sum_{\substack{\gamma \in \Gamma \sigma \backslash \Gamma / \Gamma \tau \\ c\left(\sigma \gamma \tau^{-1}\right)=c}} \bar{\kappa}(\gamma) e\left(\frac{m a\left(\sigma \gamma \tau^{-1}\right)+n d\left(\sigma \gamma \tau^{-1}\right)}{c}\right) .
$$

This definition is equivalent to

$$
K_{\sigma, \tau}(m, n, c)=\sum_{\substack{a\left(\Lambda_{\sigma}\right), d\left(\Lambda_{\tau}\right) \\
\sigma^{-1}\left(\begin{array}{l}
a * \\
c
\end{array}\right) \tau \in \Gamma}} \bar{\kappa}\left(\sigma^{-1}\left(\begin{array}{ll}
a & * \\
c & d
\end{array}\right) \tau\right) e\left(\frac{m a+n d}{c}\right) .
$$

The Kloosterman sums satisfy the Weil upper bound

$$
\left|K_{\sigma, \tau}(m, n, c)\right| \leqslant 2^{\nu(c)} \mathcal{N}(\operatorname{gcd}(m, n, c)) \mathcal{N}(c)^{1 / 2},
$$

where $\nu(c)$ is the number of prime divisors of $c$. (See [31], Prop. 5.1)

Proposition 2.3. Let $\sigma^{-1}(\infty)$ be an essential cusp of $\Gamma, m \in \Lambda_{\sigma}^{\wedge}-\{0\}$, and $f \in C^{\infty}(N \backslash G, m)$ verifying the conditions $(C)$ for some $\sigma_{0}>1$. Then $P_{f}(\sigma, g)$ belongs to $\mathrm{L}^{2}(\Gamma \backslash \mathrm{G}, \kappa)$. The Fourier expansion of $P_{f}(\sigma, g)$ at an essential cusp $\tau^{-1}(\infty)$ is given by

$$
P_{f}\left(\sigma, \tau^{-1} g\right)=\sum_{n \in \Lambda_{\tilde{\tau}}} F_{f, \sigma}(\tau, n)(g),
$$

where 


$$
F_{f, \sigma}(\tau, n)(g)=\delta_{\sigma, \tau} \delta_{m, n} f(g)+A(\tau)^{-1} \sum_{c \neq 0} K_{\sigma, \tau}(m, n, c) \mathcal{A}_{n} l_{1 / c} f(g),
$$

with the operator $\mathcal{A}_{n}$ defined as in Section 2.1.1 and where $l_{c}$ is the operator defined on $f$ by $l_{c} f(g)=f(h[c] g)$.

Proof. See [31] Proposition 4.2 for the case of trivial $K$-type and [13] (7.16) for the general case, under the conditions $\sigma=\tau=I d$. We adapt these proofs to our situation by doing simple changes.

Proposition 2.4. Let $f, m, \sigma$ as in Proposition 2.3 Assume moreover that $f$ is of type $(l, q)$. If $\phi \in C^{\infty}(\Gamma \backslash G, l, q)$ is such that $g \mapsto P_{f}(\sigma, g) \phi(g)$ is integrable on $\Gamma \backslash G$, then

$$
\left\langle P_{f}(\sigma, \cdot), \phi(\cdot)\right\rangle_{\Gamma \backslash G}=A(\sigma)\left\langle f(\cdot), F_{\phi}(\sigma, m)(\cdot)\right\rangle_{N \backslash G},
$$

where $\phi(g)$ has the Fourier expansion at $\sigma^{-1}(\infty)$

$$
\phi\left(\sigma^{-1} g\right)=\sum_{m \in \Lambda_{\sigma}^{\wedge}} F_{\phi}(\sigma, m)(g) .
$$

Proof. It is again a classical result. See [13] Lemma 7.3.1, for the case $\sigma=I d$. The adaptation to our situation is again easy to handle.

\section{Corollary 2.1.}

$$
\left\langle P_{f}(\cdot, \sigma), P_{f^{\prime}}(\cdot, \tau)\right\rangle=\delta_{\sigma, \tau} \delta_{m, n} A(\tau)\left\langle f, f^{\prime}\right\rangle_{N \backslash G}+\sum_{c \neq 0} K_{\sigma, \tau}(m, n, c)\left\langle\mathcal{A}_{n} l_{1 / c} f, f^{\prime}\right\rangle_{N \backslash G}
$$




\subsection{Summation formulae of Kuznetsov type}

In this section we come back to the case $G=\mathrm{SL}_{2}(\mathbb{C})$. We introduce two formulae, the so called Kuznetsov type formulae, which give relations between Kloosterman sums and the spectrum of the metaplectic group. They originated in the context of $\mathrm{SL}_{2}(\mathbb{Z})$, in the work of Kuznetsov [26], and were generalized in [36] to any discrete subgroup of $\mathrm{SL}_{2}(\mathbb{R})$.

For an imaginary quadratic field $k$, analogue formulae can be derived. Although the line of thought for the case $\mathrm{SL}_{2}(k)$ follows the same steps as in the real case, the ingredients needed here are more sophisticated; they are mostly taken from a recent article of Bruggeman and Motohashi ([4]) which is itself the result of a series of works developed along the last twenty years by Bruggeman, Goodman, Miatello, Motohashi, Pacharoni and Wallach. The results in [4] are proved for the field $k=\mathbb{Q}(i)$, but a generalization to any imaginary quadratic field is clearly possible; this has been recently done in [13] and we refer to it every time it is needed.

In Section 2.2.1 we shall define an analogue of the Lebedev transform, having $\mathcal{A}_{m} \varphi_{l, q}(s, p)$ as kernel; the expected properties are then derived, and we obtain a first sum formula, with the test function on the spectral side. This formula will be called spectral sum formula, and the Kloosterman sum formula (see Section2.2.3) will refer to a sum formula where the test function is on the geometric side, where Kloosterman sums occur. We insert in Section2.2.2 some estimations on the exceptional and non-exceptional spectrum of the metaplectic group.

\subsubsection{Lebedev transform and the spectral sum formula}

In this paragraph $\Gamma$ will be any subgroup of $\Gamma_{1}^{-}$containing $-I d$; our reason will become clear in Remark 2.2. For $m \in \mathbb{Z}[\omega]-\{0\}$, define $P_{l, q}(N \backslash G, m)$ as the subset of $C^{\infty}(N \backslash G, m)$, of functions satisfying the condition $(C)$ (see p. 54) and which are of type $(l, q)$. The goal is to seek some nice elements of $P_{l, q}(N \backslash G, m)$ to generate Poincaré series.

For $\sigma>0$ and $l \in \mathbb{N}$, define $\mathcal{T}_{\sigma}^{l}$ as the set of functions $\eta$ which are defined on $\{|\Re(s)| \leqslant \sigma\} \times$ $\{-l \leqslant p \leqslant l\} \subset \mathbb{C} \times \mathbb{Z}$ and which satisfy the conditions

(i) $\eta(\cdot, p)$ is holomorphic in some neighborhood of the strip $|\Re(s)| \leqslant \sigma$,

(ii) $\eta(s, p) \ll e^{-\frac{\pi}{2}|\Im(s)|}(1+|\Im(s)|)^{-a}$ for all $a>0$,

(iii) $\eta(s, p)=\eta(-s,-p)$.

The aim now is to construct an analogue $\mathcal{M}$ of the classical Lebedev transform, such that

$$
\mathcal{M}: \mathcal{T}_{\sigma}^{l} \longrightarrow P_{l, q}(N \backslash G, m) .
$$

Definition 2.6. Let $1 \leqslant \sigma, m \in \mathbb{Z}[\omega]$ and l, q a K-type. The transform $\mathcal{M}_{l, q}^{m}$, from $\mathcal{T}_{\sigma}^{l}$ to the space of functions on $G$, is defined for the element $\eta \in \mathcal{T}_{\sigma}^{l}$ by

$\mathcal{M}_{l, q}^{m} \eta(g)=\frac{1}{2 i \pi} \sum_{|p| \leqslant l} \frac{(-i)^{p}(m /|m|)^{p}}{\pi^{2}\left\|\phi_{p, q}^{l}\right\|_{K}} \int_{(0)} \eta(s, p)(2 \pi|m|)^{-s} \Gamma(l+1+s) \mathcal{A}_{m} \varphi_{l, q}(s, p)(g) s^{\epsilon(p)} \sin \pi s d s$

with $\epsilon(0)=1$ and $\epsilon(p)=-1$ if $p \neq 0$. 
All expected properties of $\mathcal{M}_{l, q}^{m}$ hold; in particular there exist some kind of inverse operator $\mathcal{L}_{l, q}^{m}$ such that:

$$
\mathcal{L}_{l, q}^{m}: P_{l, q}(N \backslash G, m) \longrightarrow\{\text { functions on } \mathbb{C} \times \mathbb{Z}\} .
$$

Definition 2.7. Let $f \in P_{l, q}(N \backslash G, m)$, and let $\sigma_{0}>0$ be the parameter controlling its growth at 0 (see the condition $(C) p$. 54). The transform $\mathcal{L}_{l, q}^{m} f$ of $f$ is defined as the function on $\{|\Re(s)| \leqslant$ $\left.\sigma_{0}\right\} \times\{p \in \mathbb{Z},|p| \leqslant l\}$ given by

$$
\mathcal{L}_{l, q}^{m} f(s, p)=\frac{(-i)^{-p}(m /|m|)^{-p}}{\pi^{2}\left\|\phi_{p, q}^{l}\right\|_{K}}(2 \pi|m|)^{s} \Gamma(l+1-s) \int_{N \backslash G} f(g) \overline{\mathcal{A}_{m} \varphi_{l, q}(-\bar{s}, p)(g)} d g .
$$

Theorem 2.3. Let $m, l, q$ be as above.

(i) Let $\eta \in \mathcal{T}_{\sigma}^{l}$. Then $\mathcal{M}_{l, q}^{m} \eta$ belongs to $P_{l, q}(N \backslash G, m)$ and, for any $0<\alpha<1$, we have in the strip $|\Re(s)|<\alpha$ that

$$
\mathcal{L}_{l, q}^{m} \mathcal{M}_{l, q}^{m} \eta(s, p)=\frac{2}{\pi} \frac{s^{\epsilon(p)+1}}{p^{2}-s^{2}} \prod_{j=1}^{l}\left(j^{2}-s^{2}\right) \eta(s, p) .
$$

(ii) Let $\eta, \theta \in \mathcal{T}_{\sigma}^{l}$ with some $1<\sigma<\frac{3}{2}$. Then

$$
\left\langle\mathcal{M}_{l, q}^{m} \eta, \mathcal{M}_{l, q}^{m} \theta\right\rangle_{N \backslash G}=\frac{1}{\pi^{3} i} \sum_{|p| \leqslant l} \int_{(0)} \eta(s, p) \bar{\theta}(s, p) \frac{s^{2 \epsilon(p)+1}}{p^{2}-s^{2}} \prod_{j=1}^{l}\left(j^{2}-s^{2}\right) d s .
$$

This is Theorem 7.1 and Lemma 7.1 of [4], generalized by Theorem 9.1.4 and Lemma 9.1.5 of [13].

We come now to the kernel of the Bessel transform. For it, we need to define a new Bessel function as product of two classical Bessel functions.

Definition 2.8. Define

$$
\begin{aligned}
\mathcal{J}_{s, p}(z) & =J_{s-p}(z) J_{s+p}(\bar{z}) \\
& =\left|\frac{z}{2}\right|^{2 s}\left(\frac{z}{|z|}\right)^{-2 p} \sum_{m, n \geqslant 0} \frac{(-1)^{m+n}(z / 2)^{2 m}(\bar{z} / 2)^{2 n}}{m 1 n ! \Gamma(s-p+m+1) \Gamma(s+p+n+1)}
\end{aligned}
$$

and

$$
\mathcal{K}_{s, p}(z)=\frac{1}{\sin \pi s}\left(\mathcal{J}_{-s,-p}(z)-\mathcal{J}_{s, p}(z)\right)
$$

Let $m, n, c \in \mathbb{C}$. To any $\eta \in \mathcal{T}_{\sigma}^{l}$ we associate the function $\kappa(m, n, c) \eta$ defined by

$$
\kappa(m, n, c) \eta:(\nu, p) \mapsto \mathcal{K}_{\nu, p}(4 \pi c \sqrt{m n}) \eta(\nu, p) .
$$

Because $\mathcal{J}_{s, p}(z)=\mathcal{J}_{-s,-p}(z)$ when $s, p \in \mathbb{Z}, \mathcal{K}_{s, p}(z)$ is holomorphic as function of $s$. 
Lemma 2.2. Let $m, n, c \in \mathbb{Z}[\omega]$, let $(l, q)$ be a $K$-type, and let $\sigma \geqslant 1$. Then, for any $\eta \in \mathcal{T}_{\sigma}^{l}$, the following equality between functions on $G$ holds:

$$
\mathcal{A}_{m} \ell_{c} \mathcal{M}_{l, q}^{n} \eta=|\pi c|^{2} \mathcal{M}_{l, q}^{m} \kappa(m, n, c) \eta
$$

This is Lemma 7.2 of [4], and Lemma 9.1.8 of [13].

The proofs of the above results actually use the explicit generator of the subspace $W_{l, q}^{\exp }(s, p, m)$ of $W_{l, q}(s, p, m)$ consisting in functions which are of exponential growth. We saw in Section 2.1.1 that its complement, $W_{l, q}^{\mathrm{pol}}(s, p, m)$ is generated by $\mathcal{A}_{m} \varphi_{l, q}(s, p)$. The Goodman-Wallch operator $\mathcal{B}_{m}$ is defined in Section 6 of [4]; it generates $W_{l, q}^{\exp }(s, p, m)$. We shall not go into details here, nevertheless, one shows that $\mathcal{A}_{m} \varphi_{l, q}(s, p)$ is expressed as combination of $\mathcal{B}_{m} \varphi_{l, q}(s, p)$ and $\mathcal{B}_{m} \varphi_{l, q}(-s,-p)$; this is $(6.15)$ of [4], from which one obtains the following formula, valid for $\alpha>0$ :

$$
\begin{gathered}
\mathcal{M}_{l, q}^{m} \eta(g)=\frac{i}{\pi} \sum_{|p| \leqslant l} \frac{(i m /|m|)^{-p}}{\left\|\Phi_{p, q}^{l}\right\|_{K}} \int_{(\alpha)} \eta(s, p)(2 \pi|m|)^{s} \Gamma(l+1+s) \mathcal{B}_{m} \varphi_{l, q}(s, p)(g) d s \\
+B(\eta) \mathcal{B}_{m} \varphi_{l, q}(1,0)(g)+\mathcal{O}\left(v^{3}\right) .
\end{gathered}
$$

The Goodman-Wallach operator satisfies the property

$$
\mathcal{B}_{m} \varphi_{l, q}(s, p)(g) \ll v^{1+\Re(s)}, \quad \text { if } g=n a[v] k,
$$

and we obtain the estimate

$$
\mathcal{M}_{l, q}^{m} \eta(g)=\mathcal{O}\left(v^{1+\alpha}+v^{2}+v^{3}\right) .
$$

Comparing with the condition $(C)$ p. 54, one sees that, as $v \rightarrow 0$, the term $\mathcal{B}_{m} \varphi_{l, q}(1,0)(g)$ causes problem with convergence. The key is to introduce a smooth function with compact support $\rho$, wich shifts the convergence issue to $\rho(g) \mathcal{B}_{m} \varphi_{l, q}(s, 0)(g)$, whose properties are then

$$
\rho(g) \mathcal{B}_{m} \varphi_{l, q}(s, 0)(g)= \begin{cases}\mathcal{O}\left(v^{1+\Re(s)}\right) & r \rightarrow 0, \\ 0 & r \rightarrow \infty .\end{cases}
$$

For $\Re(s)>1$, the condition $(C)$ is satisfied, and the associated Poincaré series $P_{\rho \mathcal{B}_{l, q}^{m}} \varphi_{l, q}(s, p)(g) \in$ $\mathrm{L}^{2}(\Gamma \backslash G, \kappa)$. Then, one has to make use of analytic continuation of this Poincaré series, as function of $s$, to the entire plane, with poles at spectral parameters. In our case, no poles occur in $] 1 / 3,1]$; this is a consequence of the cubic Shimura correspondence (see Section 2.4). The next result is to prove that the Poincaré series are square-integrable function, in our case until $\Re(s)>1 / 3$. This gives sense to $\mathcal{B}_{l, q}^{m} \varphi_{l, q}(1,0)(g)$, and thereby to the Poincaré series $P_{\mathcal{M}_{l, q}^{m}} ;$; all details can be found in [13], Section 9.2. Taking these considerations into account, one proves the expected formula:

$$
\left\langle P_{f}(\cdot, \sigma), \phi(\cdot)\right\rangle=A(\sigma)\left\langle f(\cdot), F_{\phi}(m, \cdot, \sigma)\right\rangle_{N \backslash G} .
$$

We now come to the spectral sum formula. Let us define the Bessel transform which will appear in the next theorem:

Definition 2.9. Let $\mathcal{H}_{\alpha}$ be the set of functions defined on $\{s \in \mathbb{C}:|\Re(s)| \leqslant \alpha\} \times \mathbb{Z}$ such that 
(i) $h(s, p)=h(-s,-p)$,

(ii) $h$ is holomorphic on $|\Re(s)| \leqslant \alpha$,

(iii) $h(s, p) \ll(1+|s|)^{-a}(1+|p|)^{-b}$, for some $A, b>0$.

Let $\alpha \in] \frac{1}{3}, 1\left[\right.$ and let $h \in \mathcal{H}_{\alpha}$. Define $\boldsymbol{B} h$ on $\mathbb{C}$ by

$$
\boldsymbol{B} h(z)=\frac{1}{2 \pi i} \sum_{p \in \mathbb{Z}} \int_{(0)} \mathcal{K}_{s, p}(z) h(s, p)\left(p^{2}-s^{2}\right) d s
$$

This converges absolutely for $a>2$ and $b>3$.

The spectral sum formula is a statement independent of the $K$-type $(l, q)$, that we had to carry until now. Recall that $\Gamma_{1}^{-}$was defined in 2.1.4.

Theorem 2.4. Let $\alpha \in] \frac{1}{2}, 1\left[\right.$ and let $h \in \mathcal{H}_{\alpha}$, with $a>2$ and $b>3$. Let $\Gamma$ a subgroup of $\Gamma_{1}^{-}$, and assume that $-I d \in \Gamma$. Let $m, n \in \mathbb{Z}[\omega]$. Then,

$$
\begin{gathered}
\sum_{c \neq 0} \frac{K_{\sigma, \tau}(m, n, c)}{\mathcal{N}(c)} \boldsymbol{B} h\left(\frac{4 \pi \sqrt{m n}}{c}\right)+\delta_{\sigma, \tau} \delta_{m, n} \sum_{p \in \mathbb{Z}} \int_{(0)} h(s, p)\left(p^{2}-s^{2}\right) d s \\
=\sum_{(s, p)} \sum_{f} \bar{\rho}_{f}(\sigma, m) \rho_{f}(\tau, n) h(s, p)+\frac{1}{2 i \pi} \sum_{\sigma_{i} \in \mathcal{C}(\Gamma)} \sum_{p \in \mathbb{Z}} \int_{(0)} \overline{\psi_{\sigma, \sigma_{i}}}(s, m, p) \psi_{\tau, \sigma_{i}}(s, m, p) h(s, p) d s,
\end{gathered}
$$

where the first sum in the right side is taken over the spectral parameters $(s, p)$, and for a given $(s, p)$, the sum over $f$ is taken over an orthonormal basis of the proper subspace of $\mathrm{L}^{2}\left(\Gamma \backslash \mathrm{SL}_{2}(\mathbb{C}), \kappa\right)$ corresponding to the spectral parameter $(s, p)$. The sum over the $\sigma_{i}$ 's, means the sum over all essential cusps of the group $\Gamma$.

Proof. The proof goes in two steps. The first version of the spectral sum formula is obtained in a classical way, by computing the scalar product of two Poincaré series in two different manners. The generating functions of these Poincaré series are Lebedev transforms $\mathcal{M}_{l, q}^{m} \eta$. To obtain the final version, a further step is required, namely an extension method.

In order to prove a first version of the theorem, we have to introduce the following function:

$$
\lambda_{l}(s, p)=\Gamma(l+1+s) \Gamma(l+1-s) \frac{\sin ^{2}(\pi s)}{\left(s^{2}-p^{2}\right)^{2}} s^{2 \epsilon(p)} .
$$

Let $h \in \mathcal{H}_{\alpha}$. The case where $h$ is of the form $h=\eta \bar{\theta} \lambda_{l}$ for some $\eta, \theta \in \mathcal{T}_{\alpha}^{l}$ is obtained by the usual methods above, using the formula (2.2.2). The Fourier expansion of the Poincaré series and the above properties allow us to evaluate the product of two Poincaré series. 


$$
\begin{aligned}
& \left\langle P_{\mathcal{M}^{n} \eta}(\cdot, \tau), P_{\mathcal{M}^{m} \theta}(\cdot, \sigma)\right\rangle=\left\langle\mathcal{M}^{n} \eta, F_{P_{\mathcal{M}^{m} \theta}(\cdot, \sigma)}(n, \cdot, \tau)\right\rangle \\
= & \sum_{c} K_{\sigma, \tau}(m, n, c)\left\langle\mathcal{M}^{n} \eta, \mathcal{A}_{n} \ell_{1 / c} \mathcal{M}^{m} \theta\right\rangle \\
= & \sum_{c} K_{\sigma, \tau}(m, n, c)\left|\frac{\pi}{c}\right|^{2}\left\langle\mathcal{M}^{n} \eta, \mathcal{M}^{n} \kappa(n, m, 1 / c) \theta\right\rangle \\
= & \pi^{2} \sum_{c} \frac{K_{\sigma, \tau}(m, n, c)}{|c|^{2}} \sum_{|p| \leqslant l} \int_{(0)} \eta(s, p) \overline{\kappa(n, m, 1 / c) \theta}(s, p) \frac{s}{p^{2}-s^{2}} \prod_{j=1}^{l}\left(j^{2}-s^{2}\right) d s \\
= & \pi^{2} \sum_{c} \frac{K_{\sigma, \tau}(m, n, c)}{|c|^{2}} \sum_{|p| \leqslant l} \int_{(0)} \frac{\mathcal{K}_{s, p}\left(\frac{4 \pi}{c} \sqrt{n m}\right)}{n} \eta(s, p) \bar{\theta}(s, p) \frac{s}{p^{2}-s^{2}} \prod_{j=1}^{l}\left(j^{2}-s^{2}\right) d s .
\end{aligned}
$$

Let us carry out the same scalar product, using the spectral decomposition. The formula 2.2.2 gives, for a function $f$ of type $(l, q)$ with spectral parameter $(s, p)$

$$
\begin{aligned}
\left\langle P_{\mathcal{M}^{n} \eta}(\cdot, \tau), f\right\rangle & =\left\langle\mathcal{M}^{n} \eta, \rho_{f}(n, \tau)\right\rangle_{N \backslash G} \\
& =\overline{\rho_{f}(n)}\left\langle\mathcal{M}^{n} \eta, \mathcal{A}_{n} \varphi_{l, q}\right\rangle_{N \backslash G} \quad \text { by Proposition } 2.2 \\
& =\frac{\left\|\Phi_{q}^{l}\right\|_{K}}{\Gamma(l+1+\bar{s})} \overline{\rho_{f}(n)} \mathcal{L}^{n} \mathcal{M}^{n} \eta \quad \text { by Definition2.6 } \\
& =\left\|\Phi_{q}^{l}\right\|_{K} \overline{a_{\tau}(n)} \Gamma(l+1-\bar{s}) \frac{\sin \pi \bar{s}}{\bar{s}^{2}-p^{2}} \eta \quad \text { by Theorem2.3. }
\end{aligned}
$$

Multiplying by the conjugate, we get

$$
\begin{gathered}
\left\langle P_{\mathcal{M}^{n} \eta}(\cdot, \tau), f\right\rangle\left\langle f, P_{\mathcal{M}^{m} \theta}(\cdot, \sigma)\right\rangle= \\
\|\Phi\|^{2} \overline{\rho_{f}(n, \tau)} \rho_{f}(m, \sigma) \Gamma(l+1-\bar{s}) \Gamma(l+1-s) \frac{\sin ^{2} \pi \bar{s}}{\left(\bar{s}^{2}-p^{2}\right)^{2}} \eta \bar{\theta}
\end{gathered}
$$

We then do the same with Eisenstein series, and replace, for principal series and for the continuous spectrum, $(s \in i \mathbb{R}) \Gamma(l+1-\bar{s}) \Gamma(l+1-s)$ by $\Gamma(l+1+s) \Gamma(l+1-s)$, and for the complementary series, $\Gamma(l+1-\bar{s}) \Gamma(l+1-s)$ by $\Gamma(l+1-s)^{2}$. This closes the first case, and it remains to show that the theorem is true for any function $h$ as specified.

We finish the proof using an extension method; it consists in proving Theorem 2.4 for any $h \in \mathcal{H}_{\alpha}$, from functions of the shape $h=\eta(s, p) \bar{\theta}(s, p) \lambda_{l}(s, p)$ for which the theorem has been already proved. One shows that under some convergence hypothesis, if the Theorem is true for for a sequence $f_{n}$ of functions which converges to $f$, then the theorem is valid for the function $f$. Then, one defines 


$$
\begin{aligned}
& \Lambda_{n}(s, p)= \begin{cases}\lambda_{l}(s, p) & \text { if } n \leqslant l \\
0 & \text { if } n>l\end{cases} \\
& \eta_{n}(s, p)= \begin{cases}\Lambda_{n}(s, p)^{-1} f(s, p) e^{s^{2} / n} & \text { if }|p| \leqslant n \\
0 & \text { if }|p|>n\end{cases} \\
& \theta_{n}(s, p)= \begin{cases}e^{s^{2} / n} & \text { if }|p| \leqslant n \\
0 & \text { if }|p|>b\end{cases}
\end{aligned}
$$

Let $f_{n}=\eta_{n} \overline{\theta_{n}} \Lambda_{n}$; then the sequence $\left\{f_{n}\right\}$ tends to $f$ and the theorem is true for each $f_{n}$. This finishes the proof of Theorem 2.4 .

Remark 2.2. The reason why we set the condition $-I d \in \Gamma$ is that otherwise we have to deal with $K$-types with $l$ odd. This implies also $p$ odd, and in this case one actually shows that the function $\lambda_{l}(s, p)$ is given by

$$
\lambda_{l}(s, p)=\Gamma(l+1+s) \Gamma(l+1-s) \frac{\sin ^{2} \pi(s-p)}{\left(s^{2}-p^{2}\right)^{2}} s^{2 \epsilon(p)} .
$$

This corresponds to our definition of $\lambda_{l}(s, p)$ for $p$ even, but causes the apparition of a pole of the function $\Lambda_{n}^{-1}$ for $p$ odd. This has then to be corrected by adding a complementary condition on the zeros of $h$. Nevertheless, Theorem 2.4 is valid, but with the extra condition that $h$ has a double zero at $\pm \frac{1}{2}$.

\subsubsection{On the spectrum of the metaplectic group}

In this section, we derive from the spectral sum formula some consequences on the spectrum of $\mathrm{L}^{2}\left(\Gamma \backslash \mathrm{SL}_{2}(\mathbb{C}), \kappa\right)$. This is done by choosing a suitable function $h$ in Theorem 2.4 of Section 2.2.1. and by estimating the $\delta$-term and the Kloosterman term. The first one will be evaluated directly, and the second one will be estimated through the Weil upper bound. In order to have an explicit result, one has to come back to the arithmetical setting, i.e. to fix the group $\Gamma$ and the two cusps $\sigma^{-1}(\infty)$ and $\tau^{-1}(\infty)$. Let $d \in \mathbb{Z}[\omega]$ be coprime with 3 . Then we choose $d$ primary, i.e. $d \equiv 1$ $(\bmod 3)$; according to this, we shall work with the group

$$
\begin{aligned}
\Gamma_{d} & =\left\langle\Gamma_{1},-I d\right\rangle \cap \Gamma_{0}(d) \\
& =\left\{\gamma \in \mathrm{SL}_{2}(\mathbb{Z}[\omega]): \gamma \equiv \pm I d \quad(\bmod 3), \text { and } c(\gamma) \equiv 0 \quad(\bmod d)\right\} .
\end{aligned}
$$

For $d=1$ or $d=2, \Gamma_{d}$ is not equal to the group defined in 2.1.5 and 2.1.2) of Section 2.1, but this should not cause confusion.

We shall also work with the matrices

$$
\sigma^{-1}=\left(\begin{array}{ll}
1 & 0 \\
0 & 1
\end{array}\right) \quad \text { and } \quad \tau^{-1}=\left(\begin{array}{cc}
d-1 & d-2 \\
d & d-1
\end{array}\right) .
$$

We remark that for any primary $d, \sigma^{-1}(\infty)$ and $\tau^{-1}(\infty)$ are not $\Gamma_{d}$-equivalent essential cusps of $\Gamma_{d}$. 
Lemma 2.3. With the notations of (2.2.4) and (2.2.4), the geometric Kloosterman sums defined in Definition 2.5 of Section 2.1 .3 verify

(i) $K_{\sigma, \tau}(m, n, c)= \begin{cases}K_{3}(m, n, c) & \text { if } c \equiv \pm 1 \quad(\bmod 3) \text { and } c \equiv 0 \quad(\bmod d), \\ 0 & \text { otherwise, }\end{cases}$

(ii) $\quad K_{\sigma, \sigma}(m, n, c)=K_{\tau, \tau}(m, n, c)=0 \quad$ if $c$ is not divisible by $d$.

Proof. We use the formula (2.1.13). We have $\Lambda_{\sigma}=\Lambda_{\tau}=3 \mathbb{Z}[\omega]$. Then,

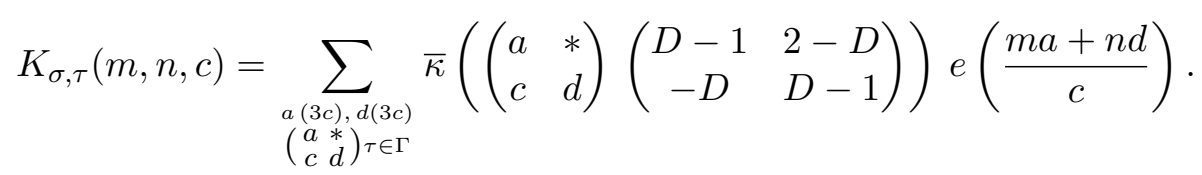

With our choice of $\Gamma$, the condition $\left(\begin{array}{ll}a & * \\ c & d\end{array}\right) \tau \in \Gamma$ means $a \equiv d \equiv 0(\bmod 3), c \equiv \pm 1(\bmod 3)$ and $c \equiv 0(\bmod D)$. Then, from the definition of $\kappa$ on $\Gamma_{2}$, one knows that $\kappa(\gamma)=\kappa\left(\gamma^{\prime} \gamma\right)$, for all $\gamma \in \Gamma_{2}, \gamma^{\prime} \in \mathrm{SL}_{2}(\mathbb{Z})$; using this, one shows that

$$
\bar{\kappa}\left(\left(\begin{array}{ll}
a & * \\
c & d
\end{array}\right)\left(\begin{array}{cc}
D-1 & 2-D \\
-D & D-1
\end{array}\right)\right)=\overline{\left(\frac{a}{c}\right)_{3}} .
$$

This proves $(i)$. The proof of $(i i)$ is similar.

Let $\left(\sigma^{-1}(\infty), \tau^{-1}(\infty)\right)$ be a couple of essential cusps of $\Gamma_{d}$, and let $m$ and $n$ be some fixed integers in $\mathbb{Z}[\omega]-\{0\}$. Define, for $d \in \mathbb{Z}[\omega]$ and for a spectral parameter $(s, p) \in \mathbb{C} \times \mathbb{Z}$,

$$
A_{m, n, \sigma, \tau}^{\mathrm{disc}}(d, s, p)=\sum_{u \in \mathcal{B}_{d}(s, p)} \rho_{u}(m, \sigma) \overline{\rho_{u}}(n, \tau),
$$

where $\mathcal{B}_{d}(s, p)$ is an orthonormal basis of the intersection of $\mathrm{L}^{2}\left(\Gamma_{d} \backslash G, \kappa\right)$ with the eigenspace of $\Delta$ of spectral parameter $(s, p)$; similarly, define then for $(s, p) \in i \mathbb{R} \times \mathbb{Z}$,

$$
A_{m, n, \sigma, \tau}^{\text {cont }}(d, s, p)=\sum_{\sigma_{i} \in \mathcal{C}\left(\Gamma_{d}\right)} \psi_{\sigma}^{\sigma_{i}}(m, s, p) \overline{\psi_{\tau}^{\sigma_{i}}}(n, s, p) .
$$

Proposition 2.5. Let $d \in \mathrm{SL}_{2}(\mathbb{Z}[\omega])$ be a primary integer. Let $\sigma^{-1}$ be one of the two matrices defined in 2.2.5. Then,

(i) Let $a>2$ and $b>2$. Then, for $X \ll 1$,

$\sum_{\substack{(s, p) \\ s \in i \mathbb{R}}} A_{n, n, \sigma, \sigma}^{\mathrm{disc}}(d, s, p)(1+|s|)^{-a}(1+|p|)^{-b}+\sum_{p} \int_{(0)} A_{n, n, \sigma, \sigma}^{\mathrm{disc}}(d, s, p)(1+|s|)^{-a}(1+|p|)^{-b} d s \ll 1$.

(ii) Let $S$ be a subset of the exceptional spectrum of $\Delta$ in $\mathrm{L}^{2}(\Gamma \backslash G, \kappa)$. Then, for $x \geqslant 1 / 2$,

$$
\sum_{s_{j} \in S} A_{n, n, \sigma, \sigma}^{\mathrm{disc}}\left(d, s_{j}, p\right) \mathcal{N}(d)^{4 x s_{j}} \ll \mathcal{N}(d)^{2 x-1} \tau(d) \log ^{2}(\mathcal{N}(d))
$$


Proof. For (i), we apply Theorem 2.4 with the special choice

$$
h(s, p)=(1+|s|)^{-a}(1+|p|)^{-b} .
$$

Theorem 2.4 gives

$$
\begin{aligned}
& \sum_{(s, p)} A_{m, m, \sigma, \sigma}^{\mathrm{disc}}(d, s, p)(1+|s|)^{-a}(1+|p|)^{-b}+\frac{1}{2 i \pi} \sum_{p \in \mathbb{Z}}(1+|p|)^{-b} \int_{(0)} A_{m, m, \sigma, \sigma}^{\mathrm{cont}}(d, s, p)(1+|s|)^{-a} d s \\
& =\sum_{p \in \mathbb{Z}} \int_{(0)} h(s, p)\left(p^{2}-s^{2}\right) d s+\sum_{c \neq 0} \frac{K_{\sigma, \sigma}(m, m, c)}{\mathcal{N}(c)} \mathbf{B} h\left(\frac{4 \pi m}{c}\right) .
\end{aligned}
$$

Evaluation of the first integral:

$$
\sum_{p \in \mathbb{Z}} \int_{(0)} h(s, p)\left(p^{2}-s^{2}\right) d s=\sum_{p \in \mathbb{Z}}(1+|p|)^{-b} \int_{0}^{\infty} \frac{p^{2}+t^{2}}{(1+t)^{a}} d t \ll 1, \text { if } a \geqslant 4 \text { and } b \geqslant 3 .
$$

Evaluation of the Kloosterman term: We begin by the transform $\mathbf{B} h(z)$. Recall that, by definition of $\mathbf{B} h$ and $\mathcal{K}_{s, p}(z)$,

$$
\begin{aligned}
\mathbf{B} h(z) & =\frac{1}{2 \pi i} \sum_{p \in \mathbb{Z}} \int_{(0)} \mathcal{K}_{s, p}(z) h(s, p)\left(p^{2}-s^{2}\right) d s \\
& =\frac{1}{\pi i} \sum_{p \in \mathbb{Z}} \int_{(0)} \mathcal{J}_{s, p}(z) h(s, p) \frac{\left(s^{2}-p^{2}\right)}{\sin \pi s} d s .
\end{aligned}
$$

There is a pole at 0 for $p \neq 0$, and by the residue theorem we have, for $\frac{1}{2}<\sigma<1$,

$$
\mathbf{B} h(z)=\frac{2}{\pi i} \sum_{p \geqslant 0} \int_{(\sigma)} \mathcal{J}_{s, p}(z) h(s, p) \frac{\left(s^{2}-p^{2}\right)}{\sin \pi s} d s+\frac{2}{\pi i} \sum_{p \geqslant 1} p^{2} \mathcal{J}_{0, p}(z) h(0, p) .
$$

(We have used the fact that $h(s, p)=h(s,-p)$.) We are working with $z$ belonging to some compact, in which case the estimate $J_{s}(z) \ll \frac{1}{\Gamma(s+1)}\left(\frac{|z|}{2}\right)^{\Re(s)}$ is valid. For the residue term, this leads to

$$
p^{2} \mathcal{J}_{0, p}(z)(1+p)^{-b} \ll p^{2}(1+p)^{-b} \frac{(|z| / 2)^{2 p}}{(p !)^{2}},
$$

and by Stirling's formula we obtain

$$
\frac{2}{\pi i} \sum_{p \geqslant 1} p^{2} \mathcal{J}_{0, p}(z) h(0, p) \ll \sum_{p \neq 0} p(1+p)^{-b}\left(\frac{|z| e}{2 p}\right)^{2 p} .
$$

For the integral over $(\sigma)$, the same estimate as above leads to $\mathcal{J}_{s, p}(z) \ll|z / 2|^{2 \Re(s)} \Gamma(s+p+$ $1)^{-1} \Gamma(s-p+1)^{-1}$, which is equal to $|z / 2|^{2 \Re(s)} \Gamma(s+p+1)^{-1}(-\pi)^{-1} \sin (\pi(s-p)) \Gamma(p-s)$. Thus we obtain 


$$
\begin{aligned}
\frac{J_{s, p}(z)}{\sin \pi s} & \ll|z / 2|^{2 \Re(s)} \frac{|\Gamma(p-s)|}{|\Gamma(s+p+1)|}=|z / 2|^{2 \Re(s)} \frac{|\Gamma(-s)|}{|\Gamma(s)||s+p|} \prod_{i=0}^{p-1} \frac{|j-s|}{|j+s|} \\
& \ll|z / 2|^{2 \Re(s)} \frac{(|s| / e)^{\Re(-s)}}{|s+p|(|s| / e)^{\Re(s)}} \ll \frac{|z / s|^{2 \Re(s)}}{|s+p|}, \quad \text { by Stirling's formula, for } \Re(s)>0 .
\end{aligned}
$$

Using this estimate in the above integral gives

$$
\begin{aligned}
\int_{(\sigma)} \mathcal{J}_{s, p}(z) h(s, p) \frac{\left(s^{2}-p^{2}\right)}{\sin \pi s} d s & \ll \int_{(\sigma)} \frac{|z|^{2 \sigma}}{|s|^{2 \sigma}}(1+|s|)^{-a}(1+p)^{-b} \frac{\left|s^{2}-p^{2}\right|}{|s+p|} d s \\
& \ll(1+p)^{-b}|z|^{2 \sigma} \int_{(\sigma)}(1+|s|)^{-a} \frac{(|s|+p)}{|s|^{2 \sigma}} d s .
\end{aligned}
$$

Since we assumed $1-2 \sigma<0$, we have $(|s|+p)|s|^{-2 \sigma} \leqslant \sigma^{-2 \sigma}(\sigma+p)$; the remaining integral converges because $a>1$ and finally, combining this estimation with the one on the residue term, we obtain

$$
\begin{aligned}
\mathbf{B} h(z) & \ll \sum_{p \geqslant 0}(1+p)^{-b}\left(\frac{|z|}{\sigma}\right)^{2 \sigma}(\sigma+p)+\sum_{p \geqslant 1} p(1+p)^{-b}\left(\frac{|z| e}{2 p}\right)^{2 p} \\
& \ll|z|^{2 \sigma} \sum_{p \geqslant 0}(1+p)^{1-b}+|z|^{2} \sum_{p \geqslant 1}(1+p)^{1-b}\left(\frac{|z| e}{2 p}\right)^{2(p-1)} \ll \mathcal{N}(z), \quad \text { for } b \geqslant 3 .
\end{aligned}
$$

The Kloosterman term can now be estimated, using Weil's upper bound. From Lemma2.3, the $c$ 's have to be divisible by $d$. Then,

$$
\begin{aligned}
& \sum_{c \equiv 0(d)} \frac{K_{\sigma, \sigma}(m, m, c)}{\mathcal{N}(c)} \mathbf{B} h\left(\frac{4 \pi m}{c}\right) \ll \sum_{c \equiv 0(d)} \mathcal{N}(c)^{-1 / 2+\epsilon} \mathbf{B} h\left(\frac{4 \pi m}{c}\right) \\
& \ll \sum_{c \equiv 0(d)} \mathcal{N}(c)^{-1 / 2+\epsilon} \mathcal{N}(m) \mathcal{N}(c)^{-1} \ll \mathcal{N}(m) \mathcal{N}(d)^{-3 / 2+\epsilon} .
\end{aligned}
$$

This proves (i). We now come to the exceptional spectrum. Note that we do not use any result as the Selberg estimate.

For (ii), we use again Theorem 2.4 and choose, for some $L>0$,

$$
h(s, p)= \begin{cases}\left(\frac{\sin (-i L s)}{L s}\right)^{4} & p=0 \\ 0 & p \neq 0\end{cases}
$$

As in the proof of (i), we obtain an upper estimate, dealing with the two terms separately.

Evaluation of the $\delta$-term: It contributes in 


$$
\begin{aligned}
& \int_{(0)} h(s, 0) s^{2} d s=\int_{0}^{\infty}\left(\frac{\sin (L t)}{i L t}\right)^{4} t^{2} d t \\
& =L^{-4} \int_{0}^{\infty} \sin ^{4}(L t) t^{-2} d t=O\left(L^{-3}\right)
\end{aligned}
$$

Evaluation of the Kloosterman term: We start as before, but because $p=0$, there is no residue of $\mathcal{J}_{s, p}(z)$, thus

$$
\int_{(\sigma)} \mathcal{J}_{s, 0}(z) h(s, 0) \frac{\left(s^{2}\right)}{\sin \pi s} d s \ll|z|^{2 \sigma} \sigma^{1-2 \sigma} \int_{(\sigma)} h(s, 0) d s
$$

and we obtain

$$
\mathbf{B} h(z) \ll|z|^{2 \sigma} \sigma^{1-2 \sigma} \int_{0}^{\infty}\left(\frac{\sin (-i L(\sigma+i t))}{L(\sigma+i t)}\right)^{4} d t .
$$

We use the following upper bound

$$
|\sin (-i L(\sigma+i t))|=\left|\frac{1}{2 i}\left(e^{L(\sigma+i t)}-e^{-L(\sigma+i t)}\right)\right| \leq e^{L \sigma}+e^{-L \sigma} \ll e^{L \sigma}
$$

to get

$$
\left|\int_{0}^{\infty}\left(\frac{\sin (-i L(\sigma+i t))}{L(\sigma+i t)}\right)^{4} d t\right| \ll \int_{0}^{\infty} \frac{e^{4 L \sigma}}{L^{4}\left(\sigma^{2}+t^{2}\right)^{2}} d t \ll \frac{e^{4 L \sigma}}{L^{4} \sigma^{3}} .
$$

Using the Weil bound, one obtains the upper bound

$$
\begin{gathered}
\sum_{(s, p)} A_{m, m, \sigma, \sigma}^{\mathrm{disc}}(d, s, p) h(s, p) \ll L^{-3}+\sum_{\substack{c \equiv 0(d) \\
c \neq 0}} \frac{K_{\sigma, \tau}(m, n, c)}{\mathcal{N}(c)} \mathcal{N}(m)^{\sigma} \mathcal{N}(c)^{-\sigma} \sigma^{1-2 \sigma} \frac{e^{4 L \sigma}}{L^{4} \sigma^{3}} \\
\ll L^{-3}+\frac{e^{4 L \sigma}}{L^{4}} \mathcal{N}(m)^{\sigma} \sum_{\substack{c \equiv 0(d) \\
c \neq 0}} \tau(c) \mathcal{N}(c)^{-1 / 2-\sigma}
\end{gathered}
$$

We estimate the last sum by

$$
\begin{gathered}
\sum_{\substack{c \equiv 0(d) \\
c \neq 0}} \tau(c) \mathcal{N}(c)^{-1 / 2-\sigma} \ll \tau(d) \mathcal{N}(d)^{-1 / 2-\sigma} \sum_{c} \tau(c) \mathcal{N}(c)^{-1 / 2-\sigma} \\
=\tau(d) \mathcal{N}(d)^{-1 / 2-\sigma} \zeta_{\mathbb{Q}(\omega)}^{2}\left(\frac{1}{2}+\sigma\right) \ll \tau(d) \mathcal{N}(d)^{-1 / 2-\sigma}\left(\sigma-\frac{1}{2}\right)^{-2},
\end{gathered}
$$

and we finally get 


$$
\sum_{(s, p)} A_{m, m, \sigma, \sigma}^{\mathrm{disc}}(d, s, p) h(s, p) \ll L^{-3}+\frac{e^{4 L \sigma}}{L^{4}} \mathcal{N}(m)^{\sigma} \tau(d) \mathcal{N}(d)^{-1 / 2-\sigma}\left(\sigma-\frac{1}{2}\right)^{-2} .
$$

We look now at a lower estimate; this is simply

$$
\sum_{(s, p)} A_{m, m, \sigma, \sigma}^{\mathrm{disc}}(d, s, p) h(s, p) \gg L^{-4} \sum_{s_{j}} A_{m, m, \sigma, \sigma}^{\mathrm{disc}}(d, s, p) e^{4 L s_{j}} .
$$

Bringing together the lower estimate and the upper estimate gives

$$
\sum_{s_{j}} A_{m, m, \sigma, \sigma}^{\mathrm{disc}}(d, s, p) e^{4 L s_{j}} \ll L+e^{4 L \sigma} \mathcal{N}(m)^{\sigma} \tau(d) \mathcal{N}(d)^{-1 / 2-\sigma}\left(\sigma-\frac{1}{2}\right)^{-2}
$$

Choose now $\sigma=\frac{1}{2}+\log ^{-1}(\mathcal{N}(d))$; this gives

$$
\sum_{s_{j}} A_{m, m, \sigma, \sigma}^{\mathrm{disc}}(d, s, p) e^{4 L s_{j}} \ll L+e^{2 L} \mathcal{N}(m)^{1 / 2} e^{4 L / \log (\mathcal{N}(d))} \tau(d) \mathcal{N}(d)^{-1} \log ^{2}(\mathcal{N}(d))
$$

If $L=4+x \log (\mathcal{N}(d)), x \geqslant 1 / 2$, one gets

$$
\sum_{\left.s_{j} \in\right] 0,1 / 3[} A_{m, m, \sigma, \sigma}^{\mathrm{disc}}(d, s, p) \mathcal{N}(d)^{4 x s_{j}} \ll \mathcal{N}(m)^{1 / 2} \mathcal{N}(d)^{2 x-1} \tau(d) \log ^{2}(\mathcal{N}(d)) .
$$

This proves assertion (ii) of the proposition.

\subsubsection{Bessel transform and the Kloosterman sum formula}

In this section we give the Kloosterman sum formula, that is, we invert the transform $\mathbf{B}$ of Theorem 2.4 by a transform $\mathbf{T}$ called inverse Bessel transform. We then study some properties of this new Bessel transform $\mathbf{T}$ that will be needed later on, in Chapter 4.

One obtains the Kloosterman sum formula by inverting the Bessel transform $\mathbf{B}$ on one side; actually $\mathbf{B}$ is not two-sided invertible. (See [13], p. 140)

Theorem 2.5. Let the transform $\boldsymbol{K}$ be defined by

$$
\boldsymbol{K} f(s, p)=\int_{\mathbb{C}^{\times}} \mathcal{K}_{s, p}(u) f(u)|u|^{-2} d u .
$$

Then, for any compactly supported function $f$ on $\mathbb{C}^{\times}, K f \in \mathcal{H}_{\alpha}, \alpha>1$, and

$$
2 \pi \boldsymbol{B} \boldsymbol{K} f=f .
$$

Moreover, the delta-term built up on Kf vanishes, see Proposition 12.3.1 of [13].

As a consequence, substituting $h$ by $\mathbf{K} f$ in Theorem 2.4 gives us the following result: 
Theorem 2.6. Let $\Gamma$ be a subgroup of $\Gamma_{1}^{-}$containing $-I d$. Let $\sigma^{-1}(\infty)$ and $\tau^{-1}(\infty)$ be two essential cusps of $\Gamma$ and $0 \neq m, n \in \Lambda_{\sigma}, \Lambda_{\tau}$ respectively. Let $f \in C_{c}^{\infty}\left(\mathbb{C}^{\times}\right)$. Then

$$
\begin{gathered}
\sum_{c} \frac{K_{\sigma, \tau}(m, n, c)}{\mathcal{N}(c)} f\left(\frac{4 \pi \sqrt{m n}}{c}\right)= \\
\sum_{(p, s)} A_{m, n, \sigma, \tau}^{\text {disc }}(d, s, p) \boldsymbol{K} f(s, p)+\frac{1}{2 i \pi} \sum_{\sigma_{i} \in \mathcal{C}(\Gamma)} \sum_{p \in \mathbb{Z}} \int_{(0)} A_{m, n, \sigma, \tau}^{\text {cont }}(d, s, p) \boldsymbol{K} f(s, p) d s .
\end{gathered}
$$

This is the final step which enabled Bruggeman and Motohashi to get their result on the fourth moment of the Dedekind zeta function. For our purpose, we shall need to estimate this transform; we possess an integral representation of the Bessel function $\mathcal{K}_{s, p}(z)$ given in the formula (12.1) of [4]

$$
\mathcal{K}_{s, p}(z)=(-1)^{p} \frac{2}{\pi} \int_{0}^{\infty} y^{2 s-1}\left(\frac{y e^{i \theta}+\left(y e^{i \theta}\right)^{-1}}{\left|y e^{i \theta}+\left(y e^{i \theta}\right)^{-1}\right|}\right)^{2 p} J_{2 p}\left(r\left|y e^{i \theta}+\left(y e^{i \theta}\right)^{-1}\right|\right) d y
$$

where we write $z=r e^{i \theta}$. The estimation at infinity $J_{0}(x) \ll x^{-1 / 2}$ implies that this formula is valid for any complex number $s$ with $|\Re(s)|<\frac{1}{4}$, and by the cubic Shimura correspondence, we know that, except for the theta term corresponding to the spectral parameter $\left(\frac{1}{3}, 0\right)$, any other exceptional parameter satisfies $s \leqslant \frac{1}{6}$.

Suppose that $f$ satisfies

$$
\frac{\partial^{a+b} f}{\partial \theta^{b} \partial r^{a}}\left(r e^{i \theta}\right) \leqslant\|f\|_{\infty} r^{-a}
$$

Proposition 2.6. Let $X \ll 1$. Let $f$ be a compactly supported function with support $[\alpha X, \beta X]$ and assume $f$ satisfies 2.2 .10 ).

(i) Let $a>2, b>2$ be some given positive integers and let $s_{0}>0$. Then for $(s, p) \in i \mathbb{R} \times \mathbb{Z}$ or $(s, p) \in[0,1] \times\{0\}$ with $s>s_{0}$, we have

$$
\boldsymbol{K} f(s, p) \ll\|f\|_{\infty} X^{\Re(s)}(1+|s|)^{-a}(1+|p|)^{-b} .
$$

(ii) For any $0<s<1 / 4$,

$$
\boldsymbol{K} f(s, 0) \ll\|f\|_{\infty} X^{-s} .
$$

Proof. For the proof of (i) we start with the expression of $\mathcal{J}_{s}(z)$ as entire series.

$$
\begin{aligned}
\left|\frac{z}{2}\right|^{-2 s}\left(\frac{z}{|z|}\right)^{2 p} \mathcal{J}_{s, p}(z) & =\left|\frac{z}{2}\right|^{-2 s}\left(\frac{z}{|z|}\right)^{2 p} J_{s-p}(z) J_{s+p}(z) \\
& =\sum_{m, n \geqslant 0} \frac{\left(-z^{2} / 4\right)^{m}\left(-\bar{z}^{2} / 4\right)^{n}}{m ! n ! \Gamma(s-p+m+1) \Gamma(s+p+n+1)} .
\end{aligned}
$$


We assume $\Re(s)>0$. Then,

$$
\begin{aligned}
\mathbf{J} f(s, p) & =\int_{\mathbb{C}^{\times}} f(z) \mathcal{J}_{s, p}(z) \frac{d z}{|z|^{2}} \\
& =\sum_{m, n \geqslant 0} \frac{\int_{\mathbb{C}^{\times}} f(z)\left|\frac{z}{2}\right|^{2 s}\left(\frac{i z}{|z|}\right)^{-2 p}\left(-z^{2} / 4\right)^{m}\left(-\bar{z}^{2} / 4\right)^{n} \frac{d z}{|z|^{2}}}{m ! n ! \Gamma(s-p+m+1) \Gamma(s+p+n+1)} \\
& =\sum_{m, n \geqslant 0} \frac{4^{-(s+m+n)}(-1)^{p+m+n} \int_{\mathbb{C}^{\times}} f(z)|z|^{2(s+m+n)}\left(\frac{z}{|z|}\right)^{-2(p-m+n)} \frac{d z}{|z|^{2}}}{m ! n ! \Gamma(s-p+m+1) \Gamma(s+p+n+1)} \\
& =\sum_{m, n \geqslant 0} \frac{4^{-(s+m+n)}(-1)^{p+m+n} \mathcal{M}_{c} f(s+m+n, p-m+n)}{m ! n ! \Gamma(s-p+m+1) \Gamma(s+p+n+1)}
\end{aligned}
$$

where $\mathcal{M}_{c} f$ is the complex Mellin transform of $f$, defined by

$$
\mathcal{M}_{c} f(s, p)=\int_{\mathbb{C}^{*}} f(u)|u|^{2 s}(u /|u|)^{-2 p}|u|^{-2} d u .
$$

It is related to the classical Mellin transform $\mathcal{M}$ by

$$
\mathcal{M}_{c} f(s, p)=2 \pi \mathcal{M} f_{p}(2 s), \text { where } f_{p}(r)=\frac{1}{2 \pi} \int_{0}^{2 \pi} f\left(r e^{i \theta}\right) e^{-2 p i \theta} d \theta .
$$

Assume now that $f$ is radial; then $f_{p}(r)=\delta_{p=0} f(r)$. We need an estimate on the Mellin transform; we have, for $a, b \geqslant 1$,

$$
\begin{aligned}
\mathcal{M} f(s) & =\int_{0}^{\infty} f(r) r^{s-1} d r=(-1)^{a} \int_{0}^{\infty} f^{(a)}(r) \frac{r^{s-1+a}}{s \ldots(s-1+a)} d r \\
& \ll\|f\|_{\infty} \frac{1}{(1+|s|)^{a}} \int_{\sqrt{\alpha X}}^{\sqrt{\beta X}} r^{s-1} d r \ll_{\alpha, \beta}\|f\|_{\infty} \frac{1}{\left|(s)_{a}\right|} X^{\Re(s) / 2} .
\end{aligned}
$$

Here we defined the product $s_{a}$ of $a$ terms by $s_{a}=s(s+1) \ldots(s+a-1)$, for $a \geqslant 1$. Inserting this estimate in 2.2.11) with $f$ radial gives, because of the supplementary condition $p-m+n=0$, the estimate for $\mathbf{J} f(s, p)$

$$
\begin{aligned}
\mathbf{J} f(s, p) & =2 \pi \sum_{n \geqslant 0} \frac{4^{-(s+|p|+2 n)} \mathcal{M} f(2(s+|p|+2 n))}{(|p|+n) ! n ! \Gamma(s+n+1) \Gamma(s+|p|+n+1)} \\
& \ll\|f\|_{\infty}\left(\frac{X}{4}\right)^{\Re(s)+|p|} \sum_{n \geqslant 0} \frac{\left(X^{2} / 16\right)^{n} \Gamma(s+n+1)^{-1} \Gamma(s+|p|+n+1)^{-1}}{(|p|+n) ! n !\left|(2(s+|p|+2 n))_{a}\right|} .
\end{aligned}
$$

We can conclude the proof of (i) in the case when $|s|$ is big, i.e. when $s=i t$ for $t$ big. In this case, the Stirling formula gives

$$
\begin{gathered}
\Gamma(s+n+1) \Gamma(s+|p|+n+1)=(s)_{n+1} \Gamma(s)(s)_{|p|+n+1} \Gamma(s) \\
=(s)_{n+1}(s)_{|p|+n+1} \frac{\Gamma(s)}{s \Gamma(-s) \sin (\pi s)} \gg s(1+t)^{2 n+|p|} \frac{\left|\frac{s}{e}\right|^{\Re(s)}}{\left|\frac{s}{e}\right|^{\Re(-s)}} e^{-\pi t} \gg(1+t)^{2 n+|p|+1} e^{-\pi t},
\end{gathered}
$$


and the sum in (2.2.12) is estimated by

$$
\sum_{n \geqslant 0} \frac{\left(X^{2} / 16\right)^{n} e^{\pi|t|}}{|p| ! n !(1+t)^{2 n+|p|+1}(1+|t|)^{a}} \ll \frac{e^{\pi|t|}}{|p| !(1+|t|)^{a}} \ll \frac{e^{\pi|t|}}{(1+|p|)^{b}(1+|t|)^{a}} .
$$

In the case when $|s|$ is small, the term corresponding to $n=0$ in the sum 2.2 .12 has a pole at $s=0$ if $p=0$. Assuming $p \neq 0$, we can still bring out from the sum

$$
\left(p !(s+|p|+2 n)_{a}\right)^{-1} \ll(1+|p|)^{-b}(1+|s|)^{-a} \ll(1+|p|)^{-b}(1+|s|)^{-a} e^{\pi|t|} .
$$

We have then proved (i) except in the case $p=0$ and $|s|$ small; we shall now prove it, together with (ii), using the formula 2.2.9] on the function $\mathcal{K}_{s, p}(z)$.

Proof of (ii) and the remaining case of (i). The integral formula for $\mathcal{K}_{s, p}(z)$ gives

$$
\begin{aligned}
\mathbf{K} f(s, 0) & =\int_{\mathbb{C}^{\times}} \mathcal{K}_{s, 0}(z) \frac{f(z)}{|z|^{2}} d z \\
& =\int_{0}^{\infty} \int_{0}^{2 \pi} y^{2 s-1} \int_{0}^{\infty} J_{0}\left(r\left|y e^{i \theta}+\left(y e^{i \theta}\right)^{-1}\right|\right) \frac{f\left(r e^{i \theta}\right)}{r} d r d \theta d y .
\end{aligned}
$$

On the one hand, the estimation $J_{0}(x) \ll 1$, for $x \ll 1$, gives

$$
\int_{0}^{\infty} J_{0}\left(r\left|y e^{i \theta}+\left(y e^{i \theta}\right)^{-1}\right|\right) \frac{f\left(r e^{i \theta}\right)}{r} d r \ll\|f\|_{\infty} \int_{\sqrt{\alpha X}}^{\sqrt{\beta X}} \frac{d r}{r} \ll_{\alpha, \beta}\|f\|_{\infty} .
$$

On the other hand, a better estimate can be derived from the integral representation of the classical Bessel function

$$
J_{0}(x)=\frac{2}{\pi} \int_{0}^{\infty} \sin (x \operatorname{ch}(t)) d t
$$

which leads us to

$$
\begin{aligned}
& \int_{0}^{\infty} J_{0}\left(r\left|y e^{i \theta}+\left(y e^{i \theta}\right)^{-1}\right|\right) \frac{f\left(r e^{i \theta}\right)}{r} d r=\frac{2}{\pi} \int_{0}^{\infty} \int_{0}^{\infty} \sin \left(r\left|y e^{i \theta}+\left(y e^{i \theta}\right)^{-1}\right| \operatorname{ch}(t)\right) \frac{f\left(r e^{i \theta}\right)}{r} d r d t \\
& =\frac{-2}{\pi} \int_{0}^{\infty} \int_{0}^{\infty} \frac{\cos \left(r\left|y e^{i \theta}+\left(y e^{i \theta}\right)^{-1}\right| c h(t)\right)}{\left|y e^{i \theta}+\left(y e^{i \theta}\right)^{-1}\right| \operatorname{ch}(t)}\left(\frac{f\left(r e^{i \theta}\right)}{r}\right)^{\prime} d r d t \\
& =\frac{-2}{\pi} \int_{0}^{\infty} \frac{1}{\left|y e^{i \theta}+\left(y e^{i \theta}\right)^{-1}\right| \operatorname{ch}(t)} \int_{\sqrt{\alpha X}}^{\sqrt{\beta X}}\left(\frac{\partial f}{\partial r}\left(r e^{i \theta}\right) r^{-1}+\frac{f}{r^{2}}\right) \cos \left(r\left|y e^{i \theta}+\left(y e^{i \theta}\right)^{-1}\right| \operatorname{ch}(t)\right) d r d t \\
& \ll\|f\|_{\infty} \int_{0}^{\infty} \frac{1}{\left|y e^{i \theta}+\left(y e^{i \theta}\right)^{-1}\right| \operatorname{ch}(t)}\left|\int_{\sqrt{\alpha X}}^{\sqrt{\beta X}} \frac{1}{r^{2}} d r\right| d t \\
& \ll \alpha, \beta \frac{\|f\|_{\infty}}{\sqrt{X}} \frac{1}{\left|y e^{i \theta}+\left(y e^{i \theta}\right)^{-1}\right|} \int_{0}^{\infty} \frac{d t}{c h(t)} \ll_{\alpha, \beta} \frac{\|f\|_{\infty} X^{-1 / 2}}{\left|y-y^{-1}\right|} .
\end{aligned}
$$

Inserting both estimates in the integral representation of $\mathbf{K} f(s, 0)$ above, one obtains

$$
\mathbf{K} f(s, 0) \ll_{\alpha, \beta}\|f\|_{\infty} \int_{0}^{\infty} y^{2 s-1} \min \left(1, \frac{X^{-1 / 2}}{\left|y-y^{-1}\right|}\right) d y .
$$


In the interval $[1 / 2,3 / 2]$, if we assume $X<4 / 9$, one verifies that $X^{-1 / 2}\left|y^{-1}-y\right|^{-1} \geqslant 1$. On $[0,1 / 2]$ and $\left[3 / 2, \infty\left[\right.\right.$, we have that $\min \left(1, \frac{X^{-1 / 2}}{\left|y-y^{-1}\right|}\right)$ is bounded respectively by $\min \left(1, X^{-1 / 2} y\right)$ and $\min \left(1, X^{-1 / 2} y^{-1}\right)$. Finally,

$$
\begin{aligned}
& \frac{\mathbf{K} f(s, 0)}{\|f\|_{\infty}} \ll_{\alpha, \beta} \int_{0}^{1 / 2} y^{2 s-1} \min \left(1, y X^{-1 / 2}\right) d y+\int_{1 / 2}^{3 / 2} y^{2 s-1} d y+\int_{3 / 2}^{\infty} y^{2 s-1} \min \left(1, X^{-1 / 2} y^{-1}\right) d y \\
& =X^{-1 / 2} \int_{0}^{\sqrt{X}} y^{2 s} d y+\int_{\sqrt{X}}^{1 / 2} y^{2 s-1} d y+O(1)+\int_{3 / 2}^{X^{-1 / 2}} y^{2 s-1} d y+X^{-1 / 2} \int_{X^{-1 / 2}}^{\infty} y^{2 s-2} d y \\
& \ll_{\alpha, \beta} X^{s}+O(1)+X^{-s} \ll_{\alpha, \beta} X^{-s}, \quad \text { as } X \ll 1 .
\end{aligned}
$$

We observe that this proves (ii) and this proves also $\mathbf{K} f(s, 0) \ll\|f\|_{\infty}$ for $s=i t$; for $t$ small, this is in agreement with (i), what was the remaining case to be proved. 


\subsection{Metaplectic group and metaplectic representations}

In this section and in the next one, we shall replace the theory of metaplectic forms in the setting of representation theory, where most of the general results have been put forward (see [20]). In this section and in Section 2.4, we shall use the notation $G=\mathrm{GL}_{2}$.

\subsubsection{Kubota symbol and metaplectic group}

We start by recalling the process of adelization of an automorphic form $f$ on a number field $k$. Let $k_{v}$ be the local field associated to $k$ at the place $v$, and $R_{v}$ be its ring of integers. For each place $v$, we have a maximal compact subgroup $K_{v}$ of $G\left(R_{v}\right)$. Let $k_{\mathbb{A}}$ be the global field.

In the classical theory of automorphic forms, we usually use the fact that

$$
\Gamma_{0}(N)=\mathrm{GL}_{2}(k) \cap \mathrm{GL}_{2}\left(k_{\infty}\right)^{+} \prod_{v} K_{v}^{N}
$$

what, according to the strong multiplicity principle, leads us to automorphic forms on $G\left(k_{\mathbb{A}}\right)$. One of the most important facts related to metaplectic forms is that the kernel of the Kubota symbol does not contain a congruence subgroup (see [22]). This is due to the fact that the Legendre symbol can always be interpreted as the character of a cubic extension of the number field $k$. A more precise statement concerning the kernel of the Kubota symbol will be given in Proposition 2.7. Consequently, it is no more expected to translate metaplectic forms as representation of the adelic group $\mathrm{GL}_{2}\left(k_{\mathbb{A}}\right)$ and we shall have to deal with automorphic forms on a covering group of $\mathrm{GL}_{2}\left(k_{\mathbb{A}}\right)$; this covering group will be defined by a cocycle suitable for our arithmetical situation. This explains, through the theory of automorphic forms, why the existence of a covering group is linked with the existence of a non congruence subgroup.

A general construction for the 2-cocycle mentioned above is done in [20], in the case of the $n$-fold covering of GL $(r)$. For $r=2$, one obtains the cocycle described earlier by Kubota in [24]. Let $v$ be a place of $k$. One needs the Hilbert symbol $(\cdot, \cdot)_{v}: k_{v} \times k_{v} \rightarrow \mu_{3}(\mathbb{C})$, whose properties are:

(i) $\left(a, b b^{\prime}\right)_{v}=(a, b)_{v}\left(a, b^{\prime}\right)_{v}$ et $\left(a a^{\prime}, b\right)_{v}=(a, b)_{v}\left(a^{\prime}, b\right)_{v}$

(ii) $(a, b)_{v}(b, a)_{v}=1$

(iii) $(a,-1)_{v}=1$

(iv) $(a, a)_{v}=1$

Similarly as in Chapter 1 p. 14, we define for $g=\left(\begin{array}{ll}a & b \\ c & d\end{array}\right) \in \mathrm{GL}_{2}\left(k_{v}\right)$, the number $x(g)$ by $x(g)=c$ if $c \neq 0$ and $x(g)=d$ if $c=0$. Let $g, h \in \mathrm{GL}_{2}\left(k_{v}\right)$; the cocycle is now explicitly given by

$$
a_{v}(g, h)=\left(\frac{x(g h)}{x(g)}, \frac{x(g h)}{x(h)}\right)_{v}\left(|g|, \frac{x(g h)}{x(g)}\right)_{v} .
$$

Now, related to the Kubota symbol $\kappa$ we define

$$
\kappa_{v}(g)=\left(c, \frac{d}{\operatorname{det}(g)}\right)_{v}, \quad \text { if } g=\left(\begin{array}{ll}
a & b \\
c & d
\end{array}\right) .
$$

Then outside the place $\lambda$, the cocycle $a$ splits over $K_{v}$, i.e. for $g_{1}, g_{2} \in K_{v}$, one has

$$
a_{v}\left(g_{1}, g_{2}\right)=\kappa_{v}\left(g_{1}\right) \kappa_{v}\left(g_{2}\right) \kappa_{v}\left(g_{1} g_{2}\right)^{-1}
$$


Actually, this formula holds for any place, under some more restrictive conditions (See [24]). In the general case, it holds at least for $g_{1}, g_{2} \in K_{v}$, such that $\left.g_{i} \equiv 1\left(\bmod n^{2}\right)\right\}$. In the cubic case, we can even reduce the condition to $g_{i} \equiv 1(\bmod 3)$; see Theorem 2 of [24].

We define a new cocycle $b$ locally by

$$
\begin{aligned}
b_{v}: \mathrm{GL}_{2}\left(k_{v}\right) \times \mathrm{GL}_{2}\left(k_{v}\right) & \rightarrow \mathbb{C} \\
\left(g_{1}, g_{2}\right) & \mapsto b_{v}\left(g_{1}, g_{2}\right)=a_{v}\left(g_{1}, g_{2}\right) k_{v}\left(g_{1}\right)^{-1} k_{v}\left(g_{2}\right)^{-1} k_{v}\left(g_{1} g_{2}\right),
\end{aligned}
$$

and globally by

$$
\begin{aligned}
b_{\mathbb{A}}: \mathrm{GL}_{2}\left(k_{\mathbb{A}}\right) \times \mathrm{GL}_{2}\left(k_{\mathbb{A}}\right) & \rightarrow \mathbb{C} \\
\left(g_{1}, g_{2}\right) & \mapsto \prod_{v} b_{v}\left(g_{1, v}, g_{2, v}\right) .
\end{aligned}
$$

Formula (2.3.3) can be reformulated as

$$
b_{v}\left(g_{1}, g_{2}\right)=1 \quad \text { for almost all } v,
$$

so that the global object $b_{\mathbb{A}}\left(g_{1}, g_{2}\right)$ has a well-defined meaning. This leads us to the notion of metaplectic group, as it was defined by Kubota. In order to simplify notations, we fix an isomorphism $\epsilon$ from $\mu_{3}\left(k_{v}\right)$ into $\mathbb{C}$, which allows us to identify $\mu_{3}\left(k_{v}\right)$ with its image in $\mathbb{C}$; let us denote these groups by $\mu_{3}$.

Definition 2.10. The local metaplectic group is a topological central extension

$$
1 \longrightarrow \mu_{3} \longrightarrow \widetilde{\mathrm{GL}_{2}}\left(k_{v}\right) \longrightarrow \mathrm{GL}_{2}\left(k_{v}\right) \longrightarrow 1,
$$

with the law of multiplication for two elements $(g, \xi)$ and $\left(g^{\prime}, \xi^{\prime}\right)$ of $\widetilde{\mathrm{GL}_{2}}\left(k_{v}\right)$ given by

$$
(g, \xi)\left(g^{\prime}, \xi^{\prime}\right)=\left(g g^{\prime}, \xi \xi^{\prime} b_{v}\left(g, g^{\prime}\right)\right) \text {. }
$$

The global metaplectic group is a topological central extension

$$
1 \longrightarrow \mu_{3} \longrightarrow \widetilde{\mathrm{GL}_{2}}\left(k_{\mathbb{A}}\right) \longrightarrow \mathrm{GL}_{2}\left(k_{\mathbb{A}}\right) \longrightarrow 1
$$

with the law of multiplication for two elements $(g, \xi)$ and $\left(g^{\prime}, \xi^{\prime}\right)$ of $\widetilde{\mathrm{GL}_{2}}\left(k_{A}\right)$ given by

$$
(g, \xi)\left(g^{\prime}, \xi^{\prime}\right)=\left(g g^{\prime}, \xi \xi^{\prime} b_{\mathbb{A}}\left(g, g^{\prime}\right)\right) .
$$

We call $\widetilde{\mathrm{GL}_{2}}\left(k_{v}\right)$ and $\widetilde{\mathrm{GL}_{2}}\left(k_{\mathbb{A}}\right)$ the local and global metaplectic group of order 3 , respectively.

As maximal compact subgroup of $\mathrm{GL}_{2}\left(k_{v}\right)$, we choose $K_{v}=\mathrm{GL}_{2}\left(R_{v}\right)$ if $|3|_{v}=1$. A special choice has to be made in the case $|3|_{v}<1$; one choose $K_{3}=\left\{g \in \mathrm{GL}_{2}\left(R_{\lambda}\right): g \equiv I d\right.$ $(\bmod 3)\}$. For the infinite place $v$, we choose $K_{v}=S U(2)$. Then $\mathrm{GL}_{2}\left(k_{\mathbb{A}}\right)$ is the group of elements in the direct product of all the $\mathrm{GL}_{2}\left(k_{v}\right)$ whose components are in $K_{v}$ for almost all places $v$ of $k$. Firstly, the compact group $K=\prod_{v} K_{v}$ and the group $\mathrm{GL}_{2}(\mathbb{C})$ have a natural injection in $\widetilde{\mathrm{GL}_{2}}\left(k_{\mathbb{A}}\right)$ given by $g \mapsto(g, 1)$. Thus we shall identify these groups (and the elements of these groups) with their image in $\widetilde{\mathrm{GL}_{2}}\left(k_{\mathbb{A}}\right)$. Secondly, there is an isomorphism $x \mapsto\left(x, \kappa_{\mathbb{A}}(x)\right)$ between $\mathrm{GL}_{2}(k)$ and its image in $\widehat{\mathrm{GL}_{2}}\left(k_{\mathbb{A}}\right)$, image which we shall denote as $\widehat{\mathrm{GL}_{2}}(k)$. Accordingly, 
for $g \in \mathrm{GL}_{2}(k)$, we define an element $\hat{g}=\left(g, s_{\mathbb{A}}(g)\right) \in \widetilde{\mathrm{GL}_{2}}\left(k_{\mathbb{A}}\right)$. Finally, we the group of cubic roots of the unity is embbeded in $\widetilde{\mathrm{GL}_{2}}\left(k_{\mathbb{A}}\right)$ through $\xi \mapsto(I d, \xi)$; we denote such an element by $i(\xi)$. Then, $\widetilde{\mathrm{GL}_{2}}\left(k_{\mathbb{A}}\right) / K_{0} \mathrm{GL}_{2}\left(k_{\infty}\right)$ is discrete, and $\widetilde{\mathrm{GL}_{2}}(k)$ is a discrete subgroup of $\widetilde{\mathrm{GL}_{2}}\left(k_{\mathbb{A}}\right)$.

We state some properties of the metaplectic group.

Proposition 2.7. $\quad$ (i) The covering $\widetilde{\mathrm{GL}_{2}}\left(k_{\mathbb{A}}\right) \rightarrow \mathrm{GL}_{2}\left(k_{\mathbb{A}}\right)$ is not trivial ([23]).

(ii) Let $\kappa$ be the Kubota symbol. With our choice of the compact subgroup $K$, if $\gamma \in \mathrm{GL}_{2}(k) \cap$ $K_{0} G_{\infty}$, then $\kappa_{\mathbb{A}}(\gamma)=\kappa(\gamma)([24]$ Proposition 1).

(iii) The kernel of the Kubota symbol $\kappa$ is given by $\widehat{\mathrm{GL}_{2}}(k) \cap K_{0} G_{\infty}=\widehat{\operatorname{ker}(\kappa)}([\overline{[24]}$ Proposition 3).

Properties (i) and (iii) express the fact that the existence of a non-congruence subgroup of finite index is equivalent to the existence of a non-trivial cavering of $\mathrm{GL}_{2}\left(k_{\mathbb{A}}\right)$.

Finally, we recall that by the strong approximation theorem, we have

$$
\mathrm{GL}_{2}\left(k_{\mathbb{A}}\right)=\mathrm{GL}_{2}(k) K \mathrm{GL}_{2}(\mathbb{C}) .
$$

For the metaplectic group, one has (see [24] Proposition 4 p. 25)

$$
\widehat{\mathrm{GL}_{2}}(k) K \mathrm{GL}_{2}(\mathbb{C}) i\left(\mu_{3}\right)=\mathrm{GL}_{2}(k) K \mathrm{GL}_{2}(\mathbb{C}) .
$$

We can check that 2.3.6 leads to automorphic forms on $\mathrm{GL}_{2}\left(k_{\infty}\right)$ with respect to the Kubota symbol $\kappa$, if we define

$$
f_{\infty}\left(g_{\infty}\right)=f_{\mathbb{A}}\left(\hat{g_{k}} k g_{\infty}\right), \quad \text { with } \hat{g_{k}} \in \widehat{\mathrm{GL}_{2}}(k), k \in K, g_{\infty} \in \mathrm{GL}_{2}\left(k_{\infty}\right) .
$$

Namely,

$$
\begin{aligned}
f\left(\gamma g_{\infty}\right) & =f_{\mathbb{A}}\left(\widehat{g_{k} \gamma^{-1}} \cdot(\gamma)_{0} k \cdot \gamma g_{\infty}\right), \text { with } g_{k} \gamma^{-1} \in G_{k},(k)_{0} k \in K \\
& =f_{\mathbb{A}}\left(\hat{g_{k}} \widehat{\gamma^{-1}} \cdot(\gamma)_{0} k \cdot \gamma g_{\infty}\right)=f_{\mathbb{A}}\left(\hat{g_{k}}\left(\gamma^{-1}, s_{\mathbb{A}}\left(\gamma^{-1}\right)\right) \cdot(\gamma) \cdot k g_{\infty}\right) \\
& =f_{\mathbb{A}}\left(\hat{g_{k}}\left(I d, s_{\mathbb{A}}\left(\gamma^{-1}\right) \cdot k g_{\infty}\right)=f_{\mathbb{A}}\left(\hat{g_{k}} k i\left(\kappa\left(\gamma^{-1}\right)\right) g_{\infty}\right)\right. \\
& \left.=f_{\mathbb{A}}\left(\hat{g_{k}} k g_{\infty}\right) \kappa\left(\gamma^{-1}\right)\right)^{-1}=\kappa(\gamma) f\left(g_{\infty}\right) .
\end{aligned}
$$

From all this we can deduce (Proposition 5 of [24]):

Proposition 2.8. There is a bijection between functions $f_{\mathbb{A}}$ on $\widetilde{\mathrm{GL}_{2}}(k) \backslash \widehat{\mathrm{GL}_{2}}(k) K_{0} \mathrm{GL}_{2}\left(k_{\infty}\right) / K i\left(\mu_{3}\right)$ satisfying $f_{\mathbb{A}}\left(\hat{g_{k}} g k \xi\right)=f_{\mathbb{A}}(g) \xi^{-1}$ and functions $f$ on $\mathbb{H}$ satisfying $f(\gamma(w))=\kappa(\gamma) f(w)$. 


\subsubsection{Metaplectic and automorphic representations}

A metaplectic representation is an automorphic representation of the metaplectic group. Let $\epsilon$ : $\mu_{3} \rightarrow \mathbb{C}$ be fixed. Since $\mu_{3}$ is the kernel of $p: \widetilde{\mathrm{GL}_{2}}\left(k_{v}\right) \rightarrow \mathrm{GL}_{2}\left(k_{v}\right)$, and since we are interested in the representations of $\widehat{\mathrm{GL}_{2}}\left(k_{v}\right)$ which do not factorise through $p$, i.e. such that $\mu_{3}$ acts injectively, we are led to make the following definition. We say that a representation $\widetilde{\pi}$ of a subgroup $H$ of $\widetilde{\mathrm{GL}_{2}}\left(k_{v}\right)$ is genuine if $\widetilde{\pi}(\xi h)=\epsilon(\xi) \widetilde{\pi}(h)$, for all $h \in H$ and $\xi \in \mu_{3}$.

In this section we shall describe the genuine metaplectic representations as well as the automorphic representations. The notations used here will be $\pi, \pi_{v}, \widetilde{\pi}$, and $\widetilde{\pi_{v}}$ for automorphic representations respectively on $G\left(k_{\mathbb{A}}\right), G\left(k_{v}\right), \widetilde{G}\left(k_{\mathbb{A}}\right)$ and $\widetilde{G}\left(k_{v}\right) . R_{v}$ will be the valuation ring of $k_{v}$, for any finite place $v$.

Definition 2.11. A representation of $\mathrm{GL}_{2}\left(k_{v}\right)$ or $\widetilde{\mathrm{GL}_{2}}\left(k_{v}\right)$ in some vector space $V$ is said to be unramified if it contains a $K_{v}$-fixed vector in $V$.

First of all, we recall that $\pi_{v}$ is of one of the following types:

- principal unramified (not square integrable), i.e. $\pi_{v}=\rho(\eta)$, with $\eta=\left(\eta_{1}, \eta_{2}\right)$ a couple of unramified characters of $k_{v}^{\times}$such that $\eta \neq\left(\eta^{\prime}|\cdot|^{ \pm 1 / 2}, \eta^{\prime}|\cdot|^{\mp 1 / 2}\right)$,

- principal ramified (not square integrable), i.e. $\pi_{v}=\rho(\eta)$, with $\eta=\left(\eta_{1}, \eta_{2}\right)$ a couple of characters of $k_{v}^{\times}$, with at least one of the two characters which is ramified, such that $\eta \neq\left(\eta^{\prime}|\cdot|^{ \pm 1 / 2}, \eta^{\prime}|\cdot|^{\mp 1 / 2}\right)$,

- special (ramified and square integrable) i.e. $\pi_{v}=\sigma(\eta)$, with $\eta=\left(\eta^{\prime}|\cdot|^{ \pm 1 / 2}, \eta^{\prime}|\cdot|^{\mp 1 / 2}\right)$ a couple of characters of $k_{v}^{\times} \cdot \sigma(\eta)$ is then defined as the irreducible subspace or subquotient of $\rho(\eta)$; its complement is a one dimensional representation, unramified if $\eta$ is unramified,

- supercuspidal (ramified and square integrable).

We now study the different types of representations $\widetilde{\pi_{v}}$ of the group $\widetilde{G}\left(k_{v}\right)$. We shall focus on principal metaplectic representations. For it, we briefly recall the construction of these representations, as described in [20]. Let $H_{v}$ the group of diagonal matrices of $G_{v}, N_{v}$ the group of unipotent upper triangular matrices, and $Z_{v}$ the group of scalar matrices. If

$$
p: \widetilde{G}\left(k_{v}\right) \rightarrow G\left(k_{v}\right), \quad(g, \xi) \mapsto g,
$$

we write $p^{-1}\left(H_{v}\right), p^{-1}\left(N_{v}\right)$ and $p^{-1}\left(Z_{v}\right)$ by $\widetilde{H_{v}}, \widetilde{N_{v}}$ and $\widetilde{Z_{v}}$ and $s(N)$ by $N^{*}$.

Then $\widetilde{H_{v}^{3}} \widetilde{Z}_{v}$ is the center of $\widetilde{H_{v}}$. Let $\widetilde{H_{*}, v}$ be a maximal abelian subgroup of $\widetilde{H_{v}}$ and consider a quasicharacter $c$ of $\widetilde{H_{v}^{3}} \widetilde{Z_{v}}$, such that $c \circ i=\varepsilon$. We extend $c$ to a quasicharacter $c^{\prime}$ of $\widetilde{H_{*, v}}$ and extend $c^{\prime}$ to $\widetilde{B_{*, v}}:=\widetilde{H_{*, v}} N^{*}$ by defining it trivial on $N^{*}$. Finally, define $\mu$ on $H$ by, $\mu\left(\left(\begin{array}{cc}h_{1} & 0 \\ 0 & h_{2}\end{array}\right)\right)=$ $\left|\frac{h_{2}}{h_{1}}\right|_{v}^{1 / 2}$, and extend it to $\widetilde{B_{*, v}}$ by $\mu(h n):=\mu(p(h))$.

Under action by right translation, we define the representation $\left(\pi\left(c^{\prime}\right), V\left(c^{\prime}\right)\right)$ as the space of functions $f: \widetilde{G_{v}} \longrightarrow \mathbb{C}$ such that

$$
\begin{aligned}
& \text { - } f(b g)=(c \mu)(b) f(g) \quad \forall b \in \widetilde{B_{*, v}}, \\
& \text { - } \exists K_{f} \subset \widetilde{G_{v}}, f \text { is right } K_{f} \text {-invariant. }
\end{aligned}
$$

It is an admissible representation of $\widetilde{G_{v}}$. 
Definition 2.12. For c as above, we define

- $c^{3}: H \longrightarrow \mathbb{C}$, by $c^{3}(h):=c\left(s\left(h^{3}\right)\right)$, and

- $c_{v}^{3}: k_{v} \longrightarrow \mathbb{C}$, by $c_{v}^{3}(x):=c^{3}\left(\left(\begin{array}{cc}x & 0 \\ 0 & x^{-1}\end{array}\right)\right)$.

Then $c$ is said unramified if $c^{3}$ is trivial on $H \cap \mathrm{GL}_{2}\left(\mathcal{O}_{k_{v}}\right)$.

Proposition 2.9. ([20], cor. 1.2.8) If $c_{v}^{3} \neq|\cdot|_{v}^{ \pm 1}$, then $V\left(c^{\prime}\right)$ is irreducible.

When $c_{v}^{3}=|\cdot|_{v}$, then $V\left(c^{\prime}\right)$ admits one irreducible subquotient.

Definition 2.13. A quasicharacter $c$ such that $c_{v}^{3}=|\cdot|_{v}$ is called exceptional. The irreducible subquotient of $V\left(c^{\prime}\right)$ is called the exceptional representation and is denoted by $V_{0}\left(c^{\prime}\right)$.

We obtain that $\widetilde{\pi_{v}}$ is of one of the following types:

- principal (not square integrable), i.e. $\widetilde{\pi_{v}}=V(c)$, with $c$ non exceptional,

- exceptional (ramified and square integrable) i.e. $\widetilde{\pi_{v}}=V_{0}(c)$, with $c$ exceptional. $V_{0}(c)$ is defined as the irreducible sub-quotient of $V(c)$,

- supercuspidal (ramified and square integrable).

Actually, the exceptional representation will appear to be of first importance for us. One of the results we shall need is due to the study in [20] of the Whittaker model of the exceptional representation.

Let $e$ be a fixed non trivial continuous character of $k_{v}$, and let $V^{*}$ be the algebraic dual of $V$, where $(\pi, V)$ is some given irreducible representation of $\widehat{\mathrm{GL}_{2}\left(k_{v}\right)}$. The dual representation $\pi^{*}$ is defined by

$$
\left(\pi^{*}(g) \cdot \lambda\right)(v)=\lambda\left(\pi\left(g^{-1}\right) \cdot v\right), \quad \text { for } v \in V \text { and } \lambda \in V^{*} .
$$

The Whittaker space of $\pi$ is the subspace of $V^{*}$ defined by

$$
\begin{aligned}
\mathrm{Wh}(\pi, e) & =\left\{\lambda \in V^{*}: \pi^{*}(n) \cdot \lambda=e(n)^{-1} \lambda, \forall n \in N\right\} \\
& =\left\{\lambda \in V^{*}: \lambda(\pi(n) \cdot v)=e(n) \lambda(v), \forall v \in V, n \in N\right\} .
\end{aligned}
$$

There is another way of seeing $\mathrm{Wh}(\pi, e)$; if $\mathcal{C}^{\infty}\left(\widetilde{\mathrm{GL}_{2}}\left(k_{v}\right), \mathbb{C}, e\right)$ denotes the space of smooth functions $f$ from $\widetilde{\mathrm{GL}_{2}}\left(k_{v}\right)$ to $\mathbb{C}$ satisfying $f(n g)=e(n) f(g)$, then each $\lambda \in \mathrm{Wh}(\pi, e)$ gives rise to an injection, called a Whittaker model for $\pi$,

$$
\begin{aligned}
t_{\lambda}: V & \longrightarrow \mathcal{C}^{\infty}\left(\widetilde{\mathrm{GL}_{2}}\left(k_{v}\right), \mathbb{C}, e\right) \\
v & \mapsto t_{\lambda}(v): g \mapsto \lambda(\pi(g) \cdot v) .
\end{aligned}
$$

In the case of an irreducible representation of $\mathrm{GL}_{n}$ one knows that the Whittaker space of $\pi$ is one-dimensional, or equivalently, that the whittaker model is unique; one consequence of this is the local multiplicity one theorem. This unicity fails for most of the genuine metaplectic representations, and more precisely, we have: 
Theorem 2.7. The dimension of the Whittaker space of an irreducible genuine metaplectic representation of $\widetilde{\mathrm{GL}_{2}}\left(k_{v}\right)$ is

(i) finite dimensional,

(ii) non-zero if $\pi$ is supercuspidal,

(iii) equal to one if $\pi$ is exceptional.

Remark 2.3. Points (i) and (ii) are true in a more genral context; they are proved for an $n$-fold covering of $\mathrm{GL}_{2}$ in [20] (Theorem I.5.2). In this situation, point (iii) is valid if and only if $r=n$ or $r=n-1$ (Corollary I.3.6 of [20]). Since we are only concerned here with $\mathrm{GL}_{2}$, this means that the Whittaker model of the exceptional representation is unique only in the cases of the 2-fold cover or 3-fold cover of $\mathrm{GL}_{2}$. The degree of the covering being the order of the Legendre symbol, we have unicity of the Whittaker model of an exceptional representation in the quadratic case (treated in Chapter 1) and in the cubic case (treated in Section 2.1] and Section 2.2 of this chapter and in Chapter 3 .

We finish this section by describing more precisely $V_{0}(c)$. Let $v$ be a fixed place of $k$, not dividing 3. Let $K_{m}$ be the subgroup of $K=\mathrm{GL}_{2}\left(R_{v}\right)$ defined by

$$
K_{m}=\left\{\left(\begin{array}{ll}
a & b \\
c & d
\end{array}\right) \in \mathrm{GL}_{2}\left(R_{v}\right): \operatorname{ord}(c) \geqslant m\right\} .
$$

The space of $K_{m}$-fixed vectors of a representation $(\pi, V)$ with central character $\mu$ is defined as

$$
V^{K_{m}}=\{v \in V: \pi(k) \cdot v=\mu(k) v\} .
$$

The vectors of $V^{K_{m}}$ correspond in some sense to automorphic forms with respect to $\Gamma_{0}\left(\pi^{m}\right)$, where $\pi$ is the prime of $\mathbb{Z}[\omega]$ corresponding to the place $v$. Then,

$$
\operatorname{dim}\left(V_{0}(c)^{K_{m}}\right)=1 \quad \text { if } m=0 \text { or } m=1 .
$$

This statement is proved in [20], Theorem I.2.9 f) and Proposition I.4.4. 


\subsection{Shimura correspondence}

The goal is to present a correspondence, in a sens which will be made precise latter, between metaplctic forms $\widetilde{f} \in \mathrm{L}^{2}(\Gamma \backslash \mathbb{H}, \kappa, \widetilde{s})$ and automorphic forms $f \in \mathrm{L}^{2}(\Gamma \backslash \mathbb{H}, s)$, and to precise some characteristics of $f$ in terms of those of $\widetilde{f}$.

Recall that the group $\Gamma_{2}$ was defined $\mathrm{p}$. 48. Let $\Gamma$ be a subgroup of $\Gamma_{2}$ and let $\chi: \Gamma \longrightarrow \mathbb{C}$ be a group homomorphism. Inside the space of functions $f: \mathbb{H} \longrightarrow \mathbb{C}$ such that

$$
f(\gamma(w))=\kappa \chi(\gamma) f(w) \quad \forall \gamma \in \Gamma,
$$

we distinguish two subspaces, the one formed by the $f$ with $\|f\|<\infty$, denoted by $\mathrm{L}^{2}(\Gamma \backslash \mathbb{H}, \kappa \chi)$ and the one of functions $f$ with $(\Delta+\widetilde{s}(2-\widetilde{s})) f=0$ for some $s \in \mathbb{C}$, denoted by $\mathcal{M}(\Gamma \backslash \mathbb{H}, \kappa \chi, \widetilde{s})$. Likewise, in the space of functions $f: \mathbb{H} \longrightarrow \mathbb{C}$ such that

$$
f(\gamma(w))=\chi(\gamma) f(w) \quad \forall \gamma \in \Gamma,
$$

we distinguish two subspaces, tho one consisting of functions $f$ such that $\|f\|<\infty$, denoted by $\mathrm{L}^{2}(\Gamma \backslash \mathbb{H}, \chi)$ and the one consisting in functions $f$ such that $(\Delta+s(2-s)) f=0$, denoted by $\mathcal{A}(\Gamma \backslash \mathbb{H}, \chi, s)$.

The spaces which are of interest for us are, on the one hand, the intersection of $L^{2}(\Gamma \backslash \mathbb{H}, \kappa \chi)$ and $\mathcal{M}(\Gamma \backslash \mathbb{H}, \kappa \chi, \widetilde{s})$, denoted by $\mathrm{L}^{2}(\Gamma \backslash \mathbb{H}, \kappa \chi, \widetilde{s})$, and on the other hand the intersection of $\mathrm{L}^{2}(\Gamma \backslash \mathbb{H}, \chi)$ and $\mathcal{A}(\Gamma \backslash \mathbb{H}, \chi, s)$, denoted by $\mathrm{L}^{2}(\Gamma \backslash \mathbb{H}, \chi, s)$.

\subsubsection{Definitions and results}

The notion used to define the correspondence is that of character of a representation. Let $G$ be a locally compact group and let $(\pi, V)$ be an admissible representation of $G$. One can extend the action of $G$ on $V$ to an action of $\mathcal{H}(G)$, the space of locally constant functions with compact support, by

$$
\pi(f) \cdot v=\int_{G} f(g) \pi(g) \cdot v d g, \quad \forall f \in \mathcal{H}(G), v \in V .
$$

The representation $(\pi, V)$ being admissible, we have that $\pi(f)$ is an operator with finite rank for any $f \in \mathcal{H}(G)$ and thus the trace of $\pi(f)$ make sense.

Recall that a distribution on a topological group $G$ is a linear form on the space $\mathcal{H}(G)$. The character of $\pi$ is then the distribution on $G$,

$$
f \mapsto \operatorname{Tr}(\pi(f)),
$$

for all $f \in \mathcal{H}(G)$. We remark that the character of $\pi$ is an invariant distribution, i.e. $\operatorname{Tr}(\pi(f))=$ $\operatorname{Tr}\left(\pi\left(f^{g}\right)\right), \forall g \in G$, where $f^{g}(x):=f\left(g x g^{-1}\right), \forall x \in G$.

In many cases, the character of a representation $\pi$ of $G$ can be given by a function on $G$, that we shall call again character of $\pi$. In our case, we refer to Theorem 7.7 of Jacquet-Langlands for the non metaplectic group, and to Lemma 2.3.1 of Flicker ([9]) for a square integrable representation of $\widehat{G L}_{2}\left(k_{v}\right)$. Moreover, the case of supercuspidal representations of $\widehat{G L}_{2}\left(k_{v}\right)$, is done in [2] p. 52, with a proof independent of the trace formula.

From this discussion we make the,

Definition 2.14. The character of $\pi$ is the local integrable function $\chi_{\pi}$ on $G$ such that

$$
\operatorname{Tr}(\pi(f))=\int_{G} f(g) \chi_{\pi}(g) d g .
$$


The preceding definition, valid for $\mathrm{GL}(2)$ and $\widetilde{\mathrm{GL}(2)}$, enable us to characterise the irreducible admissible representations of $\mathrm{GL}(2)$ and the genuine irreducible representations of $\widetilde{\mathrm{GL}(2)}$ by the value of their character on the regular elements. Moreover, one shows ([2], prop. 1.1 p. 49, $2^{\circ}$ ) ) that for a regular element $g \in \widehat{\operatorname{GL}(2)}, \chi_{\widetilde{\pi}}(g) \neq 0$ only if $g^{3}$ is regular. We are now in position to define the local correspondence. Define

$$
\Delta(g)=\left|\frac{(a-b)^{2}}{a b}\right|^{1 / 2} .
$$

Definition 2.15. Let $\widetilde{\pi}$ a genuine irreducible representation of $\widetilde{\mathrm{GL}_{2}}\left(k_{v}\right)$ with character $\chi_{\widetilde{\pi}}$, and let $\pi$ be an admissible irreducible representationof $\mathrm{GL}_{2}\left(k_{v}\right)$ with character $\chi_{\pi}$. We say that $\pi$ correspond to $\widetilde{\pi}$ if, for any regular element $g$ of $G$ of the shape $g=h^{3}$, it holds

$$
\Delta(g) \chi_{\widetilde{\pi}}\left(\left(g, s(g)^{-1}\right)\right)=\sum_{h^{3}=g} \Delta(h) \chi_{\pi}(h) .
$$

We then define the global correspondence analogously.

Theorem 2.8 (Flicker). Let $v$ be a place of $k=\mathbb{Q}(\omega)$, and let $\widetilde{\pi_{v}}$ be a genuine irreducible representation of $\widetilde{G}\left(k_{v}\right)$. Then $\widetilde{\pi_{v}}$ corresponds to some irreducible representation of $G\left(k_{v}\right)$.

(2.1) This application is an injection (thm 5.2 and cor. 5.2 of [9]).

(2.2) $\left(\pi_{v}\right.$ supercuspidal $) \Rightarrow\left(\widetilde{\pi_{v}}\right.$ supercuspidal $)($ thm 5.2 of [9] $)$

(2.3) $\left(\pi_{v}\right.$ special $) \Rightarrow\left(\widetilde{\pi_{v}}\right.$ special $)($ thm 5.2 of [9] $)$

(2.4) $\left(\widetilde{\pi_{v}}\right.$ supercuspidal $) \Rightarrow\left(\pi_{v}\right.$ supercuspidal) ( $\operatorname{cor} .5 .2$ of [9])

(2.5) $\left(\widetilde{\pi_{v}}\right.$ unramified $) \Rightarrow\left(\pi_{v}\right)$ unramified $)$. (cor 5.2)

(2.6) $\left(\widetilde{\pi_{v}} \in L^{2}\right) \Rightarrow\left(\pi_{v} \in L^{2}\right)$

Remark 2.4. In this theorem we used the fact that 3 is odd. Namely, the correspondence is proved in [9] for an n-fold covering group, but some of the above properties hold only if $n$ is odd.

\subsubsection{Classical interpretation}

The proof of the correspondence (See [9], Section 1.4) shows that the central character $\mu$ of $\pi_{v}$ is related to the central character $\widetilde{\mu}$ of $\widetilde{\pi_{v}}$ through $\mu=\widetilde{\mu}^{3}$. This is equally true at the archimedean place, although, as the Hilbert symbol is trivial on $\mathbb{C}$, the metaplectic group $\widetilde{G L}_{2}(\mathbb{C})$ is isomorphic to the direct product $\mathrm{GL}_{2}(\mathbb{C}) \times \mu_{3}$.

Theorem 2.9. Let $\widetilde{f} \in \mathrm{L}^{2}(\Gamma \backslash \mathbb{H}, \kappa, \widetilde{s})$. Then,

(i) There exists some congruence subgroup $\Gamma^{\prime}$ and some $f \in L^{2}\left(\Gamma^{\prime} \backslash \mathbb{H}, \kappa, s\right)$, such that the representation $\pi_{\widetilde{f}_{\mathbb{A}}}$ corresponds to the representation $\pi_{f_{\mathbb{A}}}$, and the following relation holds

$$
3(\widetilde{s}-1)=s-1 .
$$

(ii) If $\widetilde{s}=4 / 3$, then the Whittaker model of $\pi_{\widetilde{f_{\mathbb{A}}}}$ is one-dimensional and, with the notations of the spectral decomposition theorem, $\tilde{f} \in \mathrm{L}^{2 \mathrm{Res}}$. 
When working wiht $\mathrm{GL}_{2},($ ii $)$ of Theorem 2.9 amounts to say that the quadratic case $n=2$ and the cubic case $n=3$ are the only one whithout theoretic obstruction to the computation of the Fourier coefficients of the theta functions. We recall that, as we saw in Chapter 1 and as we shall see in Chapter 3 , theta functions are the key ingredients to tackle the problem of the asymptotic distribution of the twisted Kloosterman sum.

Since there is only one theta function for $\Gamma_{1}([32])$, we deduce from $(i i)$ that for $\Gamma_{0}^{*}(D)$ with $(D, 3)=1$, the dimension of the space generated by the metaplectic forms of minimal eigenvalue is given by

$$
\prod_{v \mid D} \operatorname{dim} V_{v}^{K_{m_{v}}} \quad \text { where } m_{v}=\operatorname{ord}_{v}(D)
$$





\section{Asymptotic behaviour of $K_{3}(m, n, c)$}

In this chapter, we try to understand the behaviour of the constant appearing in the asymptotic behaviour of the cubic Kloosterman sums. More precisely, as it will be recalled latter, we have, for any $\varepsilon>0$, that

$$
\sum_{\substack{\mathcal{N}(c) \leqslant X \\ c=0(D) \\ c \equiv 1(3)}} K_{3}(m, n, c)=C(D, m, n) X^{4 / 3}+O\left(X^{5 / 4+\varepsilon}\right),
$$

and we are interested in determining the constant $C(D, m, n)$ explicitly, and in understanding its dependence with the parameter $D$. This has been done by Livné and Patterson in [31], Theorem 1.1, for a square-free parameter, $D$. We aim at improving this theorem of Livné and Patterson by weakening the condition on the level $D$.

Before exposing the results, let us make some comments on this formula and recall some notation. First of all, the condition $c \equiv 1(\bmod 3)$ is natural, since it only means that the sum is taken over the class of integers in $R=\mathbb{Z}[\omega]$ which are not divisible by $\lambda=1-\omega$, the only prime dividing 3. By taking the class we mean to take for any of such integers, its unique associated integer, i.e. one of its multiples by a unit in $\mathcal{U}=\left\{ \pm 1, \pm \omega, \pm \omega^{2}\right\}$, which is congruent to 1 modulo 3 . This is the equivalent condition to " $c$ odd", in the quadratic case over $\mathbb{Z}$. That $c$ has to be coprime with 3 comes from the definition

$$
K_{3}(m, n, c)=\sum_{x(c)}^{\times}\left(\frac{x}{c}\right)_{3} e\left(\frac{m x+n x^{*}}{c}\right),
$$

since the Legendre symbol $(\dot{\bar{\pi}})_{3}$ is defined only for $\mathcal{N}(\pi) \equiv 1(\bmod 3)$. We recall that in the notations 3.0.1, we mean actually

$$
K_{3}(m, n, c)=\sum_{\substack{x, x^{*}(c) \\ x x^{*} \equiv 1}}\left(\frac{x}{c}\right)_{3} e\left(\frac{m x+n x^{*}}{c}\right) .
$$

From the general Kloosterman summation formula described in Chapter2, Theorem2.6, we see that the asymptotic constant is given by the sum over a orthonormal basis of the metaplectic forms with minimal eigenvalue of their $m^{\text {th }}$ and $n^{\text {th }}$ Fourier coefficients. The problem is to determine an orthonormal basis and to be able to describe the Fourier coefficients of all elements of this basis. That this second point is possible is not clear since we work with metaplectic groups, for which the Whittaker models associated to an automorphic representation is a priori not one dimensional.

In Section 3.1, where we recall the theory of Eisenstein series and cubic theta functions, and explain what is the basis problem. Our method to deal with this problem in presented in Section 3.2 where, after some technical results, we give the relations between all theta functions. These results 
3 Asymptotic behaviour of $K_{3}(m, n, c)$

allow us to derive some explicit examples in Section 3.3 


\subsection{The Kubota-Patterson theta function}

This section expands the Section 2.1.2 of Chapter2 where Eisenstein series and theta functions have been defined. Section 3.1.1 takes the point of view of Kubota, and defines Eisenstein series and gives their Fourier expansion. The Fourier coefficient are Dirichlet series whose coefficients are Gauß sums. Finally we give the Maaß-Selberg relations. The Section 3.1.2 is the heart of the matter, where cubic theta functions are defined, always following Kubota, and where their properties are given, following Patterson [32]. We finally explain how the Shimura correspondence simplifies the problem, and reformulates the problem of the asymptotic behaviour of cubic Kloosterman sums.

Unless otherwise stated, the symbol $\delta$ will be the Kronecker symbol. We recall that a cusp $\sigma^{-1}(\infty)$ of a group $\Gamma \subset \mathrm{SL}_{2}(\mathbb{Z}[\omega])$, with $\sigma \in \mathrm{SL}_{2}(\mathbb{Z}[\omega])$, is said to be essential with respect to the group homomorphism $\chi \kappa$ if its stabilizor $\Gamma_{\sigma}=\left\{\gamma \in \Gamma: \gamma\left(\sigma^{-1}(\infty)\right)=\sigma^{-1}(\infty)\right\}$ satisfies $\Gamma_{\sigma} \subset \operatorname{ker}(\chi \kappa)$. The groups we shall be interested in latter on are subgroups of

$$
\Gamma_{1}=\left\{\gamma \in \mathrm{SL}_{2}(\mathbb{Z}[\omega]): \gamma \equiv 1 \quad(\bmod 3)\right\} .
$$

We define

$$
\Gamma_{0}^{*}(D)=\left\{\gamma=\left(\begin{array}{ll}
a & b \\
c & d
\end{array}\right) \in \Gamma_{1}: c \equiv 0 \quad(\bmod D)\right\} .
$$

Lemma 3.1. Let $\Gamma=\Gamma_{0}^{*}\left(\pi^{h}\right)$, and let $\sigma=\left(\begin{array}{ll}a & b \\ c & d\end{array}\right)$. Then

$$
\Gamma_{\sigma}=\sigma^{-1}\left(\begin{array}{cc}
1 & \Lambda_{\sigma} \\
0 & 1
\end{array}\right) \sigma
$$

and $\Lambda_{\sigma}=3 \pi^{\operatorname{Max}(h-2 \ell, 0)} R$, where $\ell=\operatorname{ord}_{\pi}(c)$.

\subsubsection{The Maaß- Selberg relations}

In this section, we consider some subgroup $\Gamma$ of $\mathrm{SL}_{2}(R)$, and a character $\chi$ on $\Gamma$. A point in $\mathbb{H}$ is written $w=(z, v)$, and define $v: \mathbb{H} \rightarrow \mathbb{R}_{*}^{+}$by $v\left(\left(z_{1}, v_{1}\right)\right)=v_{1}$. Eisenstein series have already been introduced in Section 2.1.2 of Chapter2, but as we work here with trivial $K$-type, the notations simplify significantly, and we take advantage of this opportunity to give the details of the Fourier expansion of Eisenstein series.

We shall use the following Bessel function:

$$
\mathbf{K}_{s}(x)=\int_{\mathbb{C}}\left(|z|^{2}+1\right)^{-s} e(-x z) \mathrm{dz} .
$$

Remark 3.1. Recall that $e(z)=\exp \left(2 i \pi \operatorname{Tr}_{k / \mathbb{Q}}(z)\right)$. The relation with the classical Bessel function is

$$
\boldsymbol{K}_{s}(x)=\frac{(2 \pi)^{s}}{\Gamma(s)}|x|^{s-1} K_{s-1}(4 \pi|x|), \quad \text { for } x \in \mathbb{C}, x \neq 0 .
$$

Moreover, we have, for $s \in \mathbb{R}, s>1$,

$$
\boldsymbol{K}_{s}(0)=\frac{\pi}{s-1}
$$


Definition 3.1. Let $\sigma^{-1}(\infty)$ be an essential cusp of $\Gamma$. The Eisenstein series associated to $\sigma$ is defined by

$$
E_{\sigma}(w, s)=\sum_{\gamma \in \Gamma_{\sigma} \backslash \Gamma} \overline{\chi(\gamma)} v(\sigma \gamma(w))^{s}, \quad \text { for } \Re(s)>2 .
$$

We prove the formula giving its Fourier expansion. This is a special case of the one proved for any $K$-type, as treated in 2.1.2 of Chapter2.

Proposition 3.1. At an essential cusp $\tau^{-1}(\infty), E_{\sigma}(w, s)$ has the Fourier expansion

$$
E_{\sigma}\left(\tau^{-1}(w), s\right)=\delta_{\sigma, \tau} v^{s}+\sum_{\mu \in \Lambda_{\tau}} v^{2-s} \boldsymbol{K}_{s}(\mu v) \psi_{\sigma, \tau}(\mu, s) e(\mu z)
$$

where its coefficient is the Dirichlet series

$$
\psi_{\sigma, \tau}(\mu, s)=\left|\Lambda_{\tau}\right|^{-1} \sum_{0 \neq c \in R}|c|^{-2 s} \sum_{\substack{d\left(\bmod \lambda_{\tau} c\right) \\
\sigma^{-1}\left(\begin{array}{ll}
a & b \\
c & d
\end{array}\right) \tau \in \Gamma}} \overline{\chi\left(\sigma^{-1}\left(\begin{array}{ll}
a & b \\
c & d
\end{array}\right) \tau\right)} e\left(\frac{\mu d}{c}\right) .
$$

Proof. We start by splitting

$$
E_{\sigma}\left(\tau^{-1}(w), s\right)=\Sigma_{1}+\Sigma_{2},
$$

where the sum $\Sigma_{1}$ (respectively $\Sigma_{2}$ ) is the sum of the terms $\overline{\chi(\gamma)} v\left(\sigma \gamma \tau^{-1}(w)\right)^{s}$ taken over the $\gamma \in \Gamma_{\sigma} \backslash \Gamma$ such that $c\left(\sigma \gamma \tau^{-1}\right)=0$ (respectively $c\left(\sigma \gamma \tau^{-1}\right) \neq 0$ ).

Lemma 3.2. $\Sigma_{1}=\delta_{\sigma, \tau} v^{s}$.

Proof. If an element $\gamma$ such that $c\left(\sigma \gamma \tau^{-1}\right)=0$ exists, then necessarily $\sigma \gamma \tau^{-1}(\infty)=\infty$, what can be written as $\gamma\left(\tau^{-1}(\infty)\right)=\sigma^{-1}(\infty)$. The cusps are then equivalent and we recall that in this case, we make the choice $\sigma=\tau$. Let us now assume that $\sigma=\tau$, and consider some element $\gamma \in \Gamma$ such that $c\left(\sigma \gamma \sigma^{-1}\right)=0$. Then $\sigma \gamma \sigma^{-1}$ stabilizes infinity, which means that $\gamma\left(\sigma^{-1}(\infty)\right)=\sigma^{-1}(\infty)$, i.e. $\gamma \in \Gamma_{\sigma}$. Hence, there is only one left coset that we choose to be the one of the identity. It follows that $\Sigma_{1}=\overline{\chi(I d)} v\left(\sigma I d \sigma^{-1}(w)\right)^{s}=v^{s}$.

Let us come back to the sum $\Sigma_{2}$, and rearrange it as

$$
\begin{aligned}
\Sigma_{2} & =\sum_{\substack{\gamma \in \Gamma_{\sigma} \backslash \Gamma \\
c\left(\sigma \gamma \tau^{-1}\right) \neq 0}} \overline{\chi(\gamma)} v\left(\sigma \gamma \tau^{-1}(w)\right)^{s} \\
& =\sum_{\gamma^{\prime} \in \Gamma_{\sigma} \backslash \Gamma / \Gamma_{\tau}} \sum_{\substack{\gamma_{\tau} \in \Gamma_{\tau} \\
c\left(\sigma \gamma^{\prime} \gamma_{\tau} \tau^{-1}\right) \neq 0}} \overline{\chi\left(\gamma^{\prime} \gamma_{\tau}\right)} v\left(\sigma \gamma^{\prime} \gamma_{\tau} \tau^{-1}(w)\right)^{s} .
\end{aligned}
$$

From Lemma 3.1, write $\gamma_{\tau}=\tau^{-1}\left(\begin{array}{cc}1 & \lambda_{\tau} \\ 0 & 1\end{array}\right) \tau$. Then the condition $c\left(\sigma \gamma^{\prime} \gamma_{\tau} \tau^{-1}\right) \neq 0$ becomes $c\left(\sigma \gamma^{\prime} \tau^{-1}\right) \neq 0$. The same idea gives $v\left(\sigma \gamma^{\prime} \gamma_{\tau} \tau^{-1}(w)\right)=v\left(\sigma \gamma^{\prime} \tau^{-1}\left(z+\lambda_{\tau}, v\right)\right)$, and we obtain

$$
\Sigma_{2}=\sum_{\substack{\gamma \in \Gamma_{\sigma} \backslash \Gamma / \Gamma_{\tau} \\ c\left(\sigma \gamma \tau^{-1}\right) \neq 0}} \overline{\chi(\gamma)} \sum_{\lambda_{\tau} \in \Lambda_{\tau}} v\left(\sigma \gamma \tau^{-1}\left(w+\lambda_{\tau}\right)\right)^{s} .
$$


Definition 3.2. Let $\Lambda$ be a lattice in $\mathbb{C}$. Let $g \in \mathrm{SL}_{2}(R)$. Define

$$
e_{g}^{\Lambda}(w, s)=\sum_{\lambda \in \Lambda} v(g(w+\lambda))^{s}, \quad w \in \mathbb{H}, s \in \mathbb{C} .
$$

As usual, one has the double decomposition lemma, which asserts that the set of representatives $\gamma$ of $\Gamma_{\sigma} \backslash \Gamma / \Gamma_{\tau}$ with $c\left(\sigma \gamma \tau^{-1}\right) \neq 0$ is equal to set set of matrices $\sigma^{-1}\left(\begin{array}{ll}a & b \\ c & d\end{array}\right) \tau \in \Gamma$ with $c \neq 0$ and $d$ $\left(\bmod \Lambda_{\tau} c\right)^{\times}$. We have then proved that

$$
\Sigma_{2}=\sum_{0 \neq c \in R} \sum_{\substack{d\left(\bmod \Lambda_{\tau} c\right) \\
\sigma^{-1}\left(\begin{array}{ll}
a & b \\
c & d
\end{array}\right) \tau \in \Gamma}} \overline{\chi\left(\sigma^{-1}\left(\begin{array}{ll}
a & b \\
c & d
\end{array}\right) \tau\right)} e_{\left(\begin{array}{ll}
a & b \\
c & d
\end{array}\right)}^{\Lambda_{\tau}}(w, s),
$$

and it remains to prove the Fourier expansion of $e_{g}^{\Lambda}(w, s)$, for some $g=\left(\begin{array}{ll}a & b \\ c & d\end{array}\right)$.

Lemma 3.3. Let $|\Lambda|$ be the area of $\Lambda \backslash \mathbb{C}$. Then

$$
e_{g}^{\Lambda}(w, s)=|\Lambda|^{-1} \sum_{\mu \in \Lambda^{\wedge}} v^{2-s}|c|^{-2 s} e\left(\frac{\mu d}{c}\right) \boldsymbol{K}_{s}(\mu v) e(\mu z) .
$$

Proof. Being $\Lambda$-invariant, the function $e_{g}^{\Lambda}(\cdot, s)$ possesses a Fourier expansion at $\infty$ of the form

$$
e_{g}^{\Lambda}(w, s)=\sum_{\mu \in \Lambda^{\wedge}} f(\mu) e(\mu z),
$$

where the Fourier coefficient $f(\mu)$ is defined by

$$
\begin{aligned}
|\Lambda| f(\mu) & =\int_{\Lambda \backslash \mathbb{C}} \sum_{\lambda \in \Lambda} v(g(w+\lambda))^{s} e(-\mu z) d z \\
& =\int_{\Lambda \backslash \mathbb{C}} \sum_{\lambda \in \Lambda} v(g(z+\lambda, v))^{s} e(-\mu(z+\lambda)) d z, \quad \text { since } \mu \in \Lambda^{\wedge} \\
& =\int_{\mathbb{C}} v(g(z, v))^{s} e(-\mu z) d z \\
& =\int_{\mathbb{C}} v^{s}\left(|c z+d|^{2}+|c|^{2} v^{2}\right)^{-s} e(-\mu z) d z \\
& =v^{s} e\left(\frac{\mu d}{c}\right) \int_{\mathbb{C}}\left(|c z|^{2}+|c|^{2} v^{2}\right)^{-s} e(\mu z) d z \\
& =v^{-s}|c|^{-2 s} e\left(\frac{\mu d}{c}\right) \int_{\mathbb{C}}\left(\left|\frac{z}{v}\right|^{2}+1\right)^{-s} e(-u z) d z .
\end{aligned}
$$

One concludes with a change of variable.

By this lemma, the proposition is proved.

Definition 3.3. Let $A>0$. We say that $A$ is big if, for any $g \in \mathrm{SL}_{2}(\mathrm{R})$,

$$
g(C(A)) \cap C(A) \neq \emptyset \Longleftrightarrow g(\infty)=\infty .
$$


According to this definition, let us modify the function $v$ by defining

$$
v_{A}(w)=\left\{\begin{array}{ll}
v & \text { if } v(w) \leq A \\
0 & \text { if } v(w)>A
\end{array} .\right.
$$

Definition 3.4. Let $A$ be big. Then

$$
E_{\sigma}^{A}(w, s)= \begin{cases}E_{\sigma}(w, s) & \text { if } w \notin \cup_{\tau \text { essential }} \tau^{-1}(C(A)) \\ E_{\sigma}(w, s)-\delta_{\sigma, \tau} v^{s} & \text { if } w \in \tau^{-1}(C(A))\end{cases}
$$

that is to say

$$
E_{\sigma}^{A}(w, s)= \begin{cases}E_{\sigma}(w, s) & \text { if } w \notin \sigma^{-1}(C(A)) \\ E_{\sigma}(w, s)-v^{s} & \text { if } w \in \sigma^{-1}(C(A))\end{cases}
$$

Lemma 3.4.

$$
E_{\sigma}^{A}(w, s)=\sum_{\gamma \in \Gamma_{\sigma} \backslash \Gamma} \overline{\chi(\gamma)} v_{A}(\sigma \gamma(w))^{s} .
$$

Proof. We have to treat two cases. (i) If $w \notin \sigma^{-1}(C(A))$ then, by the dynamic in fuchsian groups, $\gamma(w) \notin \sigma^{-1}(C(A)), \forall \gamma \in \Gamma$. Thus $\sigma \gamma(w) \notin C(A)$, and we can replace $v(\sigma \gamma(w))$ by $v_{A}(\sigma \gamma(w))$. Then,

$$
\begin{aligned}
E_{\sigma}^{A}(w, s) & =E_{\sigma}(w, s) \\
& =\sum_{\gamma \in \Gamma_{\sigma} \backslash \Gamma} \overline{\chi(\gamma)} v(\sigma \gamma(w))^{s} \\
& =\sum_{\gamma \in \Gamma_{\sigma} \backslash \Gamma} \overline{\chi(\gamma)} v_{A}(\sigma \gamma(w))^{s} .
\end{aligned}
$$

(ii) Now, if $w \in \sigma^{-1}(C(A))$, set $w=\sigma^{-1}\left(w^{\prime}\right), w^{\prime} \in C(A)$, and by Proposition 3.1

$$
E_{\sigma}^{A}(w, s)=\sum_{\mu \in \Lambda_{\hat{\tau}}} v\left(w^{\prime}\right)^{2-s} \mathbf{K}_{s}\left(\mu v\left(w^{\prime}\right)\right) \psi_{\sigma, \tau}(\mu, s) e\left(\mu z\left(w^{\prime}\right)\right),
$$

what is, with the notations of 3.1 .2 , equal to $\Sigma_{2}$. But by definition,

$$
\begin{aligned}
\Sigma_{2} & =\sum_{\substack{\gamma \in \Gamma_{\sigma} \backslash \Gamma \\
c\left(\sigma \gamma \sigma^{-1}\right) \neq 0}} \overline{\chi(\gamma)} v\left(\sigma \gamma \sigma^{-1}\left(w^{\prime}\right)\right)^{s} \\
& =\sum_{\substack{\gamma \in \Gamma_{\sigma} \backslash \Gamma \\
c\left(\sigma \gamma \sigma^{-1}\right) \neq 0}} \overline{\chi(\gamma)} v_{A}\left(\sigma \gamma \sigma^{-1}\left(w^{\prime}\right)\right)^{s}, \quad \text { since } A \text { is big, } \\
& =\sum_{\substack{\gamma \in \Gamma_{\sigma} \backslash \Gamma \\
c\left(\sigma \gamma \sigma^{-1}\right) \neq 0}} \overline{\chi(\gamma)} v_{A}\left(\sigma \gamma \sigma^{-1}\left(w^{\prime}\right)\right)^{s}+\sum_{\substack{\gamma \in \Gamma_{\sigma} \backslash \Gamma \\
c\left(\sigma \gamma \sigma^{-1}\right)=0}} \overline{\chi(\gamma)} v_{A}\left(\sigma \gamma \sigma^{-1}\left(w^{\prime}\right)\right)^{s}, \quad \text { since the second sum is } 0, \\
& =\sum_{\gamma \in \Gamma_{\sigma} \backslash \Gamma} \overline{\chi(\gamma)} v_{A}(\sigma \gamma(w))^{s} .
\end{aligned}
$$


The sense of introducing these series is that, for $\Re(s), \Re(t)>2, E_{\sigma}^{A}(w, s) \overline{E_{\tau}^{A}(w, t)}$ is $\Gamma$-invariant and integrable on $\Gamma \backslash \mathbb{H}$. We shall now calculate

$$
\int_{\Gamma \backslash \mathbb{H}} E_{\sigma}^{A}(w, s) \overline{E_{\tau}^{A}(w, t)} d w, \quad \text { for } \Re(s)<\Re(t) .
$$

This is equal to $\left(I_{1}\right)+\left(I_{2}\right)$, with

$$
\begin{aligned}
& \left(I_{1}\right)=\int_{\Gamma \backslash \mathbb{H}}\left[E_{\sigma}^{A}(w, s)-E_{\sigma}(w, s)\right] \overline{E_{\tau}^{A}(w, t)} d w, \\
& \left(I_{2}\right)=\int_{\Gamma \backslash \mathbb{H}} E_{\sigma}(w, s) \overline{E_{\tau}^{A}(w, t)} d w .
\end{aligned}
$$

Calculation of $\left(I_{1}\right)$ : The support of $E_{\sigma}^{A}(w, s)-E_{\sigma}(w, s)$ is, as we have seen already, $\sigma^{-1}(C(A))$. By taking the quotient, what is left is

$$
\begin{aligned}
\left(I_{1}\right) & =\int_{\Gamma_{\sigma} \backslash \sigma^{-1}(C(A))}\left[E_{\sigma}^{A}(w, s)-E_{\sigma}(w, s)\right] \overline{E_{\tau}^{A}(w, t)} \mathrm{d} w \\
& =\int_{\Gamma_{\Lambda_{\sigma}} \backslash C(A)}\left[E_{\sigma}^{A}\left(\sigma^{-1}(w), s\right)-E_{\sigma}\left(\sigma^{-1}(w), s\right)\right] \overline{E_{\tau}^{A}\left(\sigma^{-1}(w), t\right)} \mathrm{d} w \\
& =-\int_{A}^{\infty} \int_{\Lambda_{\sigma} \backslash \mathbb{C}} v^{s} \overline{E_{\tau}^{A}\left(\sigma^{-1}(w), t\right)} \mathrm{d} w .
\end{aligned}
$$

Only the constant term of $E_{\tau}^{A}\left(\sigma^{-1}(w), t\right)$ contributes. By the definition of $E_{\tau}^{A}\left(\sigma^{-1}(w), t\right)$ and the Proposition 3.1, it remains

$$
\begin{aligned}
\left(I_{1}\right) & =-\int_{A}^{\infty} \int_{\Lambda_{\sigma} \backslash \mathbb{C}} v^{s} v^{2-\bar{t}} \overline{\mathbf{K}_{t}(0)} \overline{\psi_{\tau, \sigma}(0, t)} \mathrm{d} w \\
& =-\left|\Lambda_{\sigma}\right| \overline{\mathbf{K}_{t}(0)} \overline{\psi_{\tau, \sigma}(0, t)} \int_{A}^{\infty} v^{s-\bar{t}+2} v^{-3} \mathrm{~d} v \\
& =\left|\Lambda_{\sigma}\right| \overline{\mathbf{K}_{t}(0)} \overline{\psi_{\tau, \sigma}(0, t)} \frac{A^{s-\bar{t}}}{s-\bar{t}}, \quad \text { since } s<\bar{t}
\end{aligned}
$$

Calculation of $\left(I_{2}\right)$ : By the Rankin unfolding method, 


$$
\begin{aligned}
\left(I_{2}\right) & =\int_{\Gamma \backslash \mathbb{H}} E_{\sigma}(w, s) \overline{E_{\tau}^{A}(w, t)} \mathrm{d} w \\
& =\int_{\Gamma \backslash \mathbb{H}} \sum_{\Gamma_{\tau} \backslash \Gamma} \chi(\gamma) v_{A}(\tau \gamma(w))^{\bar{t}} E_{\sigma}(\gamma(w), s) \overline{\chi(\gamma)} \mathrm{d} w \\
& =\int_{\Gamma_{\tau} \backslash \mathbb{H}} v_{A}(\tau(w))^{\bar{t}} E_{\sigma}(w, s) \mathrm{d} w \\
& =\int_{\Gamma_{\Lambda_{\tau}} \backslash \mathbb{H}} v_{A}(w)^{\bar{t}} E_{\sigma}\left(\tau^{-1}(w), s\right) \mathrm{d} w \\
& =\int_{\Gamma_{\Lambda_{\tau}} \backslash \mathbb{H}-C(A)} v_{A}(w)^{\bar{t}} E_{\sigma}\left(\tau^{-1}(w), s\right) \mathrm{d} w \quad \text { since } v_{A}(w)=0 \text { if } w \in C(A) \\
& =\left|\Lambda_{\tau}\right| \int_{0}^{A} v^{\bar{t}}\left\{\delta_{\sigma, \tau} v^{s}+v^{2-s} \mathbf{K}_{s}(0) \psi_{\sigma, \tau}(0, s)\right\} v^{-3} \mathrm{~d} v \\
& =\left|\Lambda_{\tau}\right| \delta_{\sigma, \tau} \frac{A^{s+\bar{t}-2}}{s+\bar{t}-2}+\left|\Lambda_{\tau}\right| \mathbf{K}_{s}(0) \psi_{\sigma, \tau}(0, s) \frac{A^{\bar{t}-s}}{\bar{t}-s} .
\end{aligned}
$$

From this, we have derived the following theorem:

Theorem 3.1. Let $\sigma^{-1}(\infty)$ and $\tau^{-1}(\infty)$ be two essential cusps of $\Gamma$. Let $s, t>2$. Then,

$$
\begin{aligned}
\int_{\Gamma \backslash \mathbb{H}} E_{\sigma}^{A}(w, s) & \overline{E_{\tau}^{A}(w, t)} d w=\left|\Lambda_{\tau}\right| \delta_{\sigma, \tau} \frac{A^{s+\bar{t}-2}}{s+\bar{t}-2} \\
& +\left|\Lambda_{\sigma}\right| \overline{\boldsymbol{K}_{t}(0)} \overline{\psi_{\tau, \sigma}(0, t)} \frac{A^{s-\bar{t}}}{s-\bar{t}}+\left|\Lambda_{\tau}\right| \boldsymbol{K}_{s}(0) \psi_{\sigma, \tau}(0, s) \frac{A^{\bar{t}-s}}{\bar{t}-s} .
\end{aligned}
$$

\subsubsection{Properties of the cubic theta functions}

The theta functions have been defined in Section 2.1.2 of Chapter2, for any essential cusp of a given group $\Gamma \subseteq \Gamma_{2}$. They are non-cuspidal, square-integrable metaplectic forms.

Definition 3.5. The theta space $\Theta(\Gamma)$ is the space generated by the theta functions $\theta_{\sigma}$, where $\sigma^{-1}(\infty)$ runs through a set of essential cusps of $\Gamma$.

Theta functions are non cuspidal square integrable metaplectic forms, and thus we always have the inclusion $\Theta(\Gamma) \subseteq \mathrm{L}^{2}(\Gamma \backslash \mathbb{H}, \kappa)$. For example, in the quadratic case, $\theta$ functions defined by a character not totally even are cuspidal (see Serre and Stark, Theorem B p. 36). What it is not clear, but necessary for us, is whether the equality holds. We recall here part (ii) of Theorem 2.9 of Section 2.4.2 of Chapter2, 
Proposition 3.2. The subspace of the discrete spectrum of $\mathrm{L}^{2}(\Gamma \backslash \mathbb{H}, \kappa)$ corresponding to the minimal eigenvalue is

$$
\mathrm{L}^{2}\left(\Gamma \backslash \mathbb{H}, \kappa, \frac{8}{9}\right)=\Theta(\Gamma) .
$$

We can now recall the asymptotic formula of the geometric cubic Kloosterman sums:

Theorem 3.2. Let $\sigma, \tau$ be two essential cusps of $\Gamma$. Then

$$
\sum_{\mathcal{N}(c) \leqslant X} K_{\sigma, \tau}(m, n, c) \sim C X^{4 / 3} \sum_{\theta \in \mathcal{B}(\Gamma)} \rho_{\theta}(\sigma, m) \overline{\rho_{\theta}}(\tau, n),
$$

where $\mathcal{B}(\Gamma)$ is an orthonormal basis of the theta space $\Theta(\Gamma)$, and $\rho_{\theta}(\sigma, m)$ is the $m^{\text {th }}$ Fourier coefficient of $\theta(w)$ at the cusp $\sigma^{-1}(\infty)$. Here the constant $C$ is given by

$$
C=\frac{\Gamma(1 / 3)^{2}}{2(2 \pi)^{5 / 3}} \frac{\left|\Lambda_{\sigma}\right|\left|\Lambda_{\tau}\right|}{\mathcal{N}(m n)^{1 / 6}} .
$$

Proof. Let us emphasize that the asymptotic formula can be derived from the Kloosterman summation formula of Theorem 2.6 of Chapter[2, in the same way as Kuznetsov did (see [26], proof of theorem 3). Moreover, it can also be derived following the method described in Chapter[1, adapting the argument of Goldfeld and Sarnak. This was done in details in [31], Theorem 4.5, and for that reason we shall not prove it here. Both methods lead to

$$
\sum_{\mathcal{N}(c) \leqslant X} K_{\sigma, \tau}(m, n, c) \sim C X^{4 / 3} \sum_{u \in \mathcal{B}(\Gamma)} \rho_{u}(\sigma, m) \overline{\rho_{u}}(\tau, n),
$$

where $\mathcal{B}(\Gamma)$ is an orthonormal basis of the space $L^{2}\left(\Gamma \backslash \mathbb{H}, \kappa, \frac{8}{9}\right)$. The proof is then concluded by Proposition 3.2 .

As in [31], having in mind our arithmetical application, the groups of importance to us are the groups

$$
\Gamma_{0}^{*}(D)=\Gamma_{1} \cap \Gamma_{0}(D) .
$$

The link between the arithmetic cubic Kloosterman sums $K_{3}(m, n, c)$ and the geometric Kloosterman sums $K_{\sigma, \tau}(m, n, c)$ is given in Lemma2.3 in Section 2.2.2 of Chapter2. Let $D, D^{\prime}$ in $\mathbb{Z}[\omega]$, $D, D^{\prime} \equiv 1(\bmod 3)$. If $\Gamma=\Gamma_{0}^{*}\left(D D^{\prime}\right)$, then it is possible to choose two essential cusps $\sigma^{-1}(\infty)$ and $\tau^{-1}(\infty)$ so that the left hand side of the formula of Theorem 3.2 is given by

$$
\sum_{\substack{\mathcal{N}(c) \leqslant X \\ c=0(d) \\\left(c, D^{\prime}\right)=1}} K_{3}(m, n, c)
$$

We saw in Section 2.3.2 of Chapter2 that in general there is not unicity of the Whittaker model for an irreducible metaplectic representation, but (iii) of Theorem 2.7 says that however, the unicity occurs in the quadratic case and in the cubic case, and only in these cases. It is therefore theoretically possible to obtain a complete description of the Fourier coefficients $\rho(m)$ of the cubic theta functions in terms of known functions. This had been already achieved by Patterson in [32], where it was proved that $\rho(m)$ is actually a cubic Gauss sum. More precisely, the space $\Theta(1)$ is one dimensional, spanned by the function

$$
\theta_{3}(w)=v^{2 / 3}+v \sum_{m \in(3 \mathbb{Z}[\omega])^{\wedge}} \tau_{3}(m) K_{1 / 3}(4 \pi|m| v) e(m z),
$$


where $\tau_{3}$ is the function defined on $\mathbb{Q}(\omega)-\{0\}$ by

$$
\tau_{3}(m)= \begin{cases}2 \bar{g}\left(\lambda^{2}, c\right)\left|\frac{d}{c}\right| 3^{n / 2} & \text { if } m= \pm \lambda^{3 n-1} c d^{3} \\ 2 e^{-2 i \pi / 9} \bar{g}\left(\omega \lambda^{2}, c\right)\left|\frac{d}{c}\right| 3^{n / 2} & \text { if } m= \pm \omega \lambda^{3 n-1} c d^{3} \\ 2 e^{2 i \pi / 9} \bar{g}\left(\omega^{2} \lambda^{2}, c\right)\left|\frac{d}{c}\right| 3^{n / 2} & \text { if } m= \pm \omega^{2} \lambda^{3 n-1} c d^{3} \\ 2 \bar{g}(1, c)\left|\frac{d}{c}\right| 3^{n / 2} & \text { if } m= \pm \lambda^{3 n-3} c d^{3} \\ 0 & \text { otherwise. }\end{cases}
$$

Actually we can be more precise about the number of Fourier coefficients of theta functions that we need. The periodicity theorem ([20], Theorem I.2.9 e)), combined with Hecke theory, allows us to obtain the following properties of a theta function

$$
\theta(w)=\sum_{m} \rho(m) K_{1 / 3}(4 \pi|m| v) e(m z) .
$$

Theorem 3.3. Let $c$ be an integer coprime with a prime $\pi$. Then,

$$
\begin{aligned}
\rho\left(\pi^{3} c\right) & =\mathcal{N}(\pi) \rho(c), \\
\rho(\pi c) & =\mathcal{N}(\pi) G(\pi) \rho(c), \\
\rho\left(\pi^{2} c\right) & =0 .
\end{aligned}
$$

Let $D, D^{\prime} \in \mathbb{Z}[\omega]$ be two integers, coprime with 3 . We conclude this section with a discussion on the constant $c\left(D, D^{\prime}\right)$ appearing in the asymptotic formula

$$
\sum_{\substack{\mathcal{N}(c) \leqslant X \\\left(c, D^{\prime}\right)=1 \\ c \equiv 1(3)}} K_{3}(m, n, c) \sim c\left(D, D^{\prime}, m, n\right) X^{4 / 3},
$$

derived from Theorem 3.2 by 3.1.3). By Theorem 3.3. $C\left(D, D^{\prime}, m, n\right)$ can be explicitly determined once we know $\mathcal{B}\left(\Gamma_{0}^{*}\left(D D^{\prime}\right)\right)$. For example, when $D$ and $D^{\prime}$ are square-free, $c\left(D, D^{\prime}, m, n\right)$ is given by Theorem 1.1 of [31]; in this case, $\Theta\left(\Gamma_{0}^{*}\left(D D^{\prime}\right)\right)$ is of dimension 1 , generated by the cubic theta function $\theta_{3}$. What has to be done to treat the general case, is to study relations between theta functions.

From the Maaß-Selberg relations, we deduce that the scalar product between two theta functions $\theta_{\sigma}$ and $\theta_{\tau}$ associated to two essential cusps of a group $\Gamma$ is given by

$$
\left\langle\theta_{\sigma}, \theta_{\tau}\right\rangle=3 \pi \operatorname{Res}_{s=4 / 3}\left(\sum_{0 \neq c \in R}|c|^{-2 s} \sum_{\substack{d(\bmod \lambda \tau c) \\
\sigma^{-1}\left(\begin{array}{ll}
a & b \\
c & d
\end{array}\right) \tau \in \Gamma}} \bar{\kappa}\left(\sigma^{-1}\left(\begin{array}{ll}
a & b \\
c & d
\end{array}\right) \tau\right)\right) .
$$

To prove this, it suffices to take the residue at $s=4 / 3$ and $t=\frac{4}{3}$ in the formula of the Theorem 3.1 Formula (3.1.6) has to be worked out in details, to give precise relations between theta functions. 


\subsection{Relations between theta functions}

To simplify notations, we write $\Theta(D)$ for $\Theta\left(\Gamma_{0}^{*}(D)\right)$ and $\mathcal{B}(D)$ for $\mathcal{B}\left(\Gamma_{0}^{*}(D)\right)$. In this section, we study the space $\Theta(D)$ and look for an orthonormal basis $\mathcal{B}(D)$ of $\Theta(D)$, when $D$ is a prime power $D=\pi^{h}$, for $h \geqslant 1$. Here $\pi$ is a prime in $R$, that we assume different from the prime $\lambda$ and from the prime 2 , and that, as such, we can and do choose such that $\pi \equiv 1(\bmod 3)$. After some preliminary results in Section 3.2.1, the relations between theta functions are given in Section 3.2.2. Some examples of $\mathcal{B}(D)$ will be provided in Section 3.3 in the cases $h=1,2,3$.

\subsubsection{Auxiliary results}

Equation 3.1.6) of Section 3.1.2 will be our main tool for the evaluation of the scalar product of theta functions. In this section, we make this formula easier to evaluate by giving some simple lemmas that will be used latter on. Since we have attached a theta function to each essential cusp of a group $\Gamma \subset \Gamma_{2}$, the next step will be to determine all $\Gamma_{0}^{*}\left(\pi^{h}\right)$-inequivalent essential cusps. We achieve this in Lemma3.5. In Lemma3.6, we work out the summation condition appearing in the formula (3.1.6), then in Lemma 3.7 we deal with some Gauß sums similar to those appearing in the inner sum of (3.1.6) and finally, in Lemma 3.8 we evaluate a Dirichlet series.

For $0 \leqslant \ell \leqslant h$, let us define $\mathcal{P}_{\ell}$ as

$$
\mathcal{P}_{\ell}= \begin{cases}1 & \text { if } \ell=0, \\ \left(R / \pi^{h-\ell} R\right)^{\times} & \text {if } 1 \leqslant l \leqslant h-1, \\ 1 & \text { if } \ell=h .\end{cases}
$$

Since we assume $\pi \neq \lambda$, we shall assume that the elements of $\mathcal{P}_{\ell}$ are chosen congruent to 1 modulo 3. Besides, for a given $m \in R$ specified in the context, we shall use the notation $\bar{x}$ for a representative of the inverse modulo $m$ of an element $x$ coprime with $m$. Finally, we define $T_{m}=\left(\begin{array}{cc}1 & 0 \\ m & 1\end{array}\right)$ and $T^{m}=\left(\begin{array}{cc}1 & m \\ 0 & 1\end{array}\right)$.

Lemma 3.5. A set of essential cusps (not necessarily inequivalent) of $\Gamma_{0}^{*}\left(\pi^{h}\right)$ is given by

$$
\left\{\frac{1}{\alpha \pi^{\ell} \nu}: \alpha \in\{ \pm 1,3\}, 0 \leqslant \ell \leqslant h, \nu \in \mathcal{P}_{\ell}\right\} \cup\left\{\frac{1}{\pi^{\ell} \nu}-1: 0 \leqslant \ell \leqslant h, \nu \in \mathcal{P}_{\ell}\right\} .
$$

Proof. Let $P=\frac{a}{c}$ be a cusp of $\Gamma_{0}^{*}\left(\pi^{h}\right)$, with $a, c \in \mathbb{Z}[\omega], \operatorname{gcd}(a, c)=1$. Since $\left(\begin{array}{cc}1 & 0 \\ 3 \pi^{h} & 1\end{array}\right) \in$ $\Gamma_{0}^{*}\left(\pi^{h}\right)$, the cusp $P$ is equivalent to the cusp $\left(\begin{array}{cc}1 & 0 \\ 3 \pi^{h} & 1\end{array}\right)(P)=\frac{a}{3 \pi^{h} a+c}$, and this allow us to assume that $\operatorname{ord}_{\pi}(c) \leqslant h$. According to this, we define $j=\operatorname{ord}_{\pi}(c), 0 \leqslant j \leqslant h$. First of all, we determine the three types of cusps of $\Gamma_{0}^{*}\left(\pi^{h}\right)$, and then rule out some of them which are not essential.

Case 1: $\pi \mid c$ and $\lambda \nmid \backslash a$. Multiplying by some unit of $\mathbb{Z}[\omega]$ if necessary, we assume that $a \equiv 1$ $\left(\bmod \lambda^{2}\right)$. Let $b, d, l$ such that $a d-b c \lambda^{2} \pi^{l}=1$, for a parameter $l \geqslant 0$ to be chosen latter on. Then, we remark that $d \equiv 1(\bmod 3)$ and that

$$
\gamma:=\left(\begin{array}{cc}
a & b \lambda^{2} \pi^{l} \\
c & d
\end{array}\right) \in \mathrm{SL}_{2}(R)
$$

Define 
- a representative $\alpha$ of the class of $c$ modulo $\lambda^{2}$ such that $\alpha$ is coprime to $\pi$,

- a representative $\nu$ of the class of $\bar{\alpha} c \pi^{-j} a$ modulo $\pi^{h-j}$ such that $\nu \equiv 1(\bmod 3)$.

Then

$$
\gamma T_{-\alpha \pi^{j} \nu}=\left(\begin{array}{cc}
a-\alpha b \lambda^{2} \pi^{l+j} \nu & b \lambda^{2} \pi^{l} \\
c-\alpha d \pi^{j} \nu & d
\end{array}\right) \equiv\left(\begin{array}{ll}
1 & 0 \\
0 & 1
\end{array}\right) \quad(\bmod 3),
$$

and $c-\alpha d \pi^{j} \nu \equiv c-c a d\left(\bmod \pi^{h}\right)$; but $c-c a d=c(1-a d)=-b c^{2} \lambda^{2} \pi^{l}$, and thus $\operatorname{ord}_{\pi}(c-c a d)=2 j+l$. We now choose $l \geqslant h-2 j$ and conclude that $\gamma T_{-\alpha \pi^{j} \nu} \in \Gamma_{0}^{*}\left(\pi^{h}\right)$. Therefore, we have that $P=\frac{a}{c}=\gamma(\infty)=\gamma T_{-\alpha \pi^{j} \nu}\left(T_{\alpha \pi^{j} \nu}(\infty)\right)$ is equivalent to $T_{\alpha \pi^{j} \nu}(\infty)=$ $\left(\alpha \pi^{j} \nu\right)^{-1}$.

Case 2: $\pi \mid c$ et $\lambda \mid a$. Multiplying by some unit if necessary, we assume that $c \equiv 1\left(\bmod \lambda^{2}\right)$. Let $b, d$ be such that $a d-b c=1$ and $d \equiv 1(\bmod 3)$. Then we remark that $a-b \equiv 1(\bmod 3)$ and that

$$
\gamma:=\left(\begin{array}{ll}
a & b \\
c & d
\end{array}\right) \in \mathrm{SL}_{2}(R)
$$

Define $\nu$ to be a representative of the class of $c \pi^{-j} \bar{d}$ modulo $\pi^{h-j}$, then

$$
\gamma T_{-\pi^{j} \nu}=\left(\begin{array}{ll}
a-b \pi^{j} \nu & b \\
c-d \pi^{j} \nu & d
\end{array}\right) \equiv\left(\begin{array}{ll}
1 & b \\
0 & 1
\end{array}\right) \quad(\bmod 3)
$$

and $c-d \pi^{j} \nu \equiv 0\left(\bmod \pi^{h}\right)$. Let $u$ be the unit of $R$ representative of the class of $b$ modulo 3 ; then $\gamma T_{-\pi^{j} \nu} T^{-u} \in \Gamma_{0}^{*}\left(\pi^{h}\right)$ and thus $P=\gamma(\infty)=\gamma T_{-\pi^{j} \nu} T^{-u}\left(T^{u} T_{\pi^{j} \nu}(\infty)\right)$ is equivalent to $\left(\pi^{j} \nu\right)^{-1}+u$. Finally we remark that $b \equiv a-1(\bmod 3)$, thus if $a$ is divisible by $\lambda^{2}$, we have $u=-1$, and otherwise (since $a$ is at least divisible by $\lambda$ ) we have $u=-\omega$ or $=-\omega^{2}$.

Case 3: $\pi \nmid c$ and $\lambda \nmid \backslash$. As before, assume $a \equiv 1\left(\bmod \lambda^{2}\right)$. Let $\alpha$ be a representative of $c$ modulo 3 such that $\alpha$ is coprime with $\pi$. Consider $b, d$ such that $a d-b c \lambda^{2}=1$ and such that $d \equiv \bar{\alpha} c$ $\left(\bmod \pi^{h}\right)$. Then $d \equiv 1(\bmod 3), \gamma:=\left(\begin{array}{cc}a & b \lambda^{2} \\ c & d\end{array}\right) \in \mathrm{SL}_{2}(R)$ and

$$
\gamma T_{-\alpha}=\left(\begin{array}{cc}
a-b \alpha \lambda^{2} & b \lambda^{2} \\
c-d \alpha & d
\end{array}\right) \in \Gamma_{1}
$$

and $c-\alpha d \equiv 0\left(\bmod \pi^{h}\right)$. Thus $\gamma T_{-\alpha} \in \Gamma_{0}^{*}\left(\pi^{h}\right)$ and $P=\gamma(\infty)=\gamma T_{-\alpha}\left(T_{\alpha}(\infty)\right)$ is equivalent to $\frac{1}{\alpha}$.

Case 4: $\pi \backslash c$ and $\lambda \mid a$. Assume $c \equiv-1\left(\bmod \lambda^{2}\right)$. Let $\alpha$ be a representative of $-a$ modulo 3 , and consider $b, d$ such that $a d \pi^{h} \lambda^{2}-b c=1$. Then $b \equiv 1(\bmod 3), \gamma:=\left(\begin{array}{ll}a & b \\ c & d \pi^{h} \lambda^{2}\end{array}\right) \in \operatorname{SL}_{2}(R)$ and

$$
\gamma S=\left(\begin{array}{cc}
b & -a \\
d \pi^{h} \lambda^{2} & -c
\end{array}\right) \equiv\left(\begin{array}{cc}
1 & \alpha \\
0 & 1
\end{array}\right) \quad(\bmod 3) .
$$

Finally, $P=\gamma S T^{-\alpha}\left(T^{\alpha} S(\infty)\right)$ is equivalent to $\alpha$.

Define the set $\mathcal{C}=\left\{ \pm 1, \pm \omega, \pm \omega^{2}, \pm \lambda, 3\right\}$ of representatives modulo 3 . From the previous discussion, we conclude that a cusp $P$ belongs to one of the following types:

type 1 , if $P \sim \frac{1}{\alpha \pi^{\ell} \nu}$, with $0 \leqslant \ell \leqslant h-1, \nu \in \mathcal{P}_{\ell}$, and $\alpha \in \mathcal{C}$, (case 1 and case 3),

type 2, if $P \sim u+\frac{1}{\pi^{\ell} \nu}$, with $0 \leqslant \ell \leqslant h-1, \nu \in \mathcal{P}_{\ell}$ and $u \in\left\{-1,-\omega,-\omega^{2}\right\}$ (case 2), 
type 3, if $P \sim \alpha$, with $\alpha \in\{ \pm \lambda, 3\}$ (case 4).

We now have to specify which of these cusps are essential. Recall that $\sigma^{-1}(\infty)$ is an essential cusp if $\kappa\left(\gamma_{\sigma}\right)=1$, for all $\gamma_{\sigma} \in \Gamma_{\sigma}$; we saw in Lemma 3.1 that such $\gamma_{\sigma}$ can be written as $\gamma_{\sigma}=\sigma^{-1}\left(\begin{array}{cc}1 & \lambda_{\sigma} \\ 0 & 1\end{array}\right) \sigma$ with $\lambda_{\sigma} \in \Lambda_{\sigma} \subseteq 3 R$.

If $\sigma^{-1}(\infty)$ is of type 1, i.e. $\sigma^{-1}=\left(\begin{array}{ll}1 & 0 \\ c & 1\end{array}\right)$, with $c=\alpha \pi^{\ell} \nu$, the condition for $\sigma^{-1}(\infty)$ to be essential is

$$
\left(\frac{c^{2} \lambda_{\sigma}}{1-c \lambda_{\sigma}}\right)_{3}=1, \quad \forall \lambda_{\sigma} \in \lambda_{\sigma}
$$

By periodicity we have

$$
\left(\frac{c^{2} \lambda_{\sigma}}{1-c \lambda_{\sigma}}\right)_{3}=\left(\frac{-c\left(1-c \lambda_{\sigma}-1\right)}{1-c \lambda_{\sigma}}\right)_{3}=\left(\frac{c}{1-c \lambda_{\sigma}}\right)_{3}
$$

Now, write $\alpha= \pm \omega^{l} \lambda^{i} \in \mathcal{C}$ and $\pi^{\ell} \nu=c^{\prime}$, i.e. $c= \pm \omega^{l} \lambda^{i} c^{\prime}$. Then $\left(\frac{c}{1-c \lambda_{\sigma}}\right)_{3}=\left(\frac{\omega}{1-c \lambda_{\sigma}}\right)_{3}^{l}\left(\frac{\lambda}{1-c \lambda_{\sigma}}\right)_{3}^{i}$, by reciprocity. We also write $c=c_{1}+\omega c_{2}$, as well as $\lambda_{\sigma}=3 r$, with $r=r_{1}+\omega r_{2}$. Then $1-c \lambda_{\sigma}=1+3\left(c_{2} r_{2}-c_{1} r_{1}+\omega\left(c_{2} r_{2}-c_{1} r_{2}-c_{2} r_{1}\right)\right)$ and by the complementary reciprocity law (see Theorem 2.1 of Chapter2), we obtain

$$
\left(\frac{c^{2} \lambda_{\sigma}}{1-c \lambda_{\sigma}}\right)_{3}=\omega^{-l\left(2 c_{2} r_{2}-c_{1} r_{1}-c_{1} r_{2}-c_{2} r_{1}\right)} \omega^{i\left(c_{2} r_{2}-c_{1} r_{1}\right)}
$$

This expression has to be 1 for any choice of $r$. Taking $r_{1} \equiv 0(\bmod 3)$ and $r_{2} \equiv 1(\bmod 3)$, we obtain $\omega^{-2 l c_{2}+l c_{1}+i c_{2}}$, and taking $r_{1} \equiv 1(\bmod 3)$ and $r_{2} \equiv 0(\bmod 3)$, we obtain $\omega^{l c_{2}+l c_{1}-i c_{1}}$, and taking the quotient, we obtain the condition $w^{i\left(c_{1}+c_{2}\right)}=1$.

If $i \neq 0$ (i.e. $\alpha \in\{ \pm \lambda, 3\})$, then we need $c_{1}+c_{2} \equiv 0(\bmod 3)$, condition under which (3.2.2) simplifies in $\omega^{i c_{2}\left(r_{1}+r_{2}\right)}$. Since $r_{1}$ and $r_{2}$ are free parameters, we conclude $c_{1} \equiv c_{2} \equiv 0(\bmod 3)$, i.e. $\alpha=3$. It is also clear that for $c \equiv 0(\bmod 3)$, the right side of $(3.2 .2)$ is equal to 1 .

If $i=0$, then $(3.2 .2)$ is equal to $\omega^{l\left(-2 c_{2} r_{2}+c_{1} r_{1}+c_{1} r_{2}+c_{2} r_{1}\right)}=\omega^{l\left(c_{1}+c_{2}\right)\left(r_{1}+r_{2}\right)}$. If $l \not \equiv 0$ $(\bmod 3)\left(\right.$ i.e. $\left.\alpha= \pm \omega, \pm \omega^{2}\right)$, then $c_{1}+c_{2} \equiv 0(\bmod 3)$, and as above we conclude that $c \equiv 0$ ( $\bmod 3$ ), which contradicts $i=0$. Thus $l=0$ (i.e. $\alpha= \pm 1$ ), which is a sufficient condition. We conclude that a cusp $\frac{1}{\alpha \pi^{\ell} \nu}$ of the first type is essential if and only if $\alpha \in\{ \pm 1,3\}$.

If $\sigma^{-1}(\infty)$ is of type 2, i.e. $\sigma^{-1}=\left(\begin{array}{ll}1 & u \\ 0 & 1\end{array}\right)\left(\begin{array}{cc}1 & 0 \\ \pi^{j} \nu & 1\end{array}\right)$ with $u \in\left\{-1,-\omega,-\omega^{2}\right\}$, the condition for $\sigma^{-1}(\infty)$ to be essential is $\left(\frac{\pi^{2 j} \nu^{2} \lambda_{\sigma}}{1-\pi^{j} \nu \lambda_{\sigma}\left(1+\pi^{j} \nu u\right)}\right)_{3}=1$ for all $\lambda_{\sigma} \in \Lambda_{\sigma}$. By choosing $\lambda_{\sigma}=\lambda^{2} r$ with $r \equiv 1(\bmod 3)$, we obtain the condition

$$
\left(\frac{\pi^{2 j} \nu^{2} r}{1-\pi^{j} \nu \lambda_{\sigma}\left(1+\pi^{j} \nu u\right)}\right)_{3}\left(\frac{\lambda^{2}}{1-\pi^{j} \nu \lambda_{\sigma}\left(1+\pi^{j} \nu u\right)}\right)_{3}=1,
$$

which, by reciprocity law, amounts to $\left(\frac{\lambda}{1+3 \pi^{j} \nu r\left(1+\pi^{j} \nu u\right)}\right)_{3}=1$, and by the complementary reciprocity law, we need $1+u=x_{1}+\omega x_{2}$, with $x_{1} \equiv 0(\bmod 3)$; among the set $-1,-\omega,-\omega^{2}$, only $u=-1$ satisfies this condition.

If $\sigma^{-1}(\infty)$ is of type 3, i.e. $\sigma^{-1}=\left(\begin{array}{cc}\alpha & -1 \\ 1 & 0\end{array}\right)$ with $\alpha \in \mathcal{C}$, then $\sigma^{-1}(\infty)$ is essential if and only if $\left(\frac{\lambda_{\sigma}}{1-\alpha \lambda_{\sigma}}\right)_{3}=1$ for any $\lambda_{\sigma} \in \Lambda_{\sigma}$. We have $\left(\frac{\lambda_{\sigma}}{1-\alpha \lambda_{\sigma}}\right)_{3}=\left(\frac{\alpha}{1-\alpha \lambda_{\sigma}}\right)_{3}^{2}\left(\frac{\lambda_{\sigma} \alpha}{1-\alpha \lambda_{\sigma}}\right)_{3}=\left(\frac{\alpha}{1-\alpha \lambda_{\sigma}}\right)_{3}^{2}$. For $\alpha \in\{ \pm \lambda, 3\}$, one sees that this expression is equal to one only in the case where $\alpha=3$.

To finish the proof of Lemma 3.5, we merely have to notice that the only essential cusp of the third type, namely $P=3$, is equivalent to the cusp $P=0$, which correspond to the cusp of second type $\left(\pi^{\ell} \nu\right)^{-1}-1$ for $\ell=0$. 
After having determined the set of essential cusps of the group $\Gamma_{0}^{*}\left(\pi^{h}\right)=\Gamma_{1} \cap \Gamma_{0}\left(\pi^{h}\right)$, and before coming back to our initial problem, i.e. to work out the scalar product of two theta functions, let us define some new notations. To any parameter $\xi=(\ell, \nu)$ with $0 \leqslant \ell \leqslant h$ and $\nu \in \mathcal{P}_{\ell}$ (as defined in (3.2.1), correspond four essential cusps as defined in Lemma3.6, and associated to them four theta functions, namely

$$
\theta_{\alpha \xi}:=\theta_{\sigma}, \quad \text { where } \quad \sigma=\left(\begin{array}{cc}
1 & 0 \\
\alpha \pi^{\ell} \nu & 1
\end{array}\right), \quad \text { for } \alpha \in\{ \pm 1,3\}
$$

and

$$
\theta_{\xi}^{\prime}:=\theta_{\sigma}, \quad \text { where } \quad \sigma=\left(\begin{array}{cc}
1 & -1 \\
\pi^{\ell} \nu & 1
\end{array}\right)\left(\begin{array}{cc}
1 & 0 \\
\alpha \pi^{\ell} \nu & 1
\end{array}\right)
$$

Therefore, a generating system of the space $\Theta\left(\pi^{h}\right)$ is given by

$$
V\left(\pi^{h}\right):=\left\{\theta_{\xi}, \theta_{-\xi}, \theta_{3 \xi}, \theta_{\xi}^{\prime}: \xi=(\ell, \nu) \text { with } 0 \leqslant \ell \leqslant h \text { and } \nu \in \mathcal{P}_{\ell}\right\} .
$$

The method for extracting a basis from this set is to come to simple relations between theta functions, using the formula 3.1.6 of Section 3.1.2. This will be achieved in various steps; at the present, we give some useful results that we shall apply in the next section. We have seen that the lattice $\Lambda_{\sigma}$ associated to the cusp $\sigma^{-1}(\infty)$ only depends on $\operatorname{ord}_{\pi}(c(\sigma))$. In particular, it is the same for all four theta functions attached to some couple $(\ell, \nu)$ in $(3.2 .3)$ and (3.2.4). More precisely, we showed in Lemma 3.1 of Section 3.1 that $\Lambda_{\ell}=3 \pi^{\operatorname{Max}(0, h-2 \ell)} R$.

Lemma 3.6. Let $\alpha_{1}, \alpha_{2} \in\{ \pm 1,3\}, \ell_{1}, \ell_{2} \in \mathbb{Z}$ such that $0 \leqslant \ell_{1}, \ell_{2} \leqslant h$, and $\nu_{1} \in \mathcal{P}_{\ell_{1}}, \nu_{2} \in \mathcal{P}_{\ell_{2}}$. Assume that $\ell_{1} \leqslant \ell_{2}$. Then

$$
\begin{aligned}
& \left\langle\theta_{\alpha_{1} \pi^{\ell_{1}} \nu_{1}}, \theta_{\alpha_{2} \pi^{\ell_{2} \nu_{2}}}\right\rangle=\operatorname{Res}_{s=4 / 3}\left(\sum_{c \equiv \alpha_{2}-\alpha_{1}(3)}^{\prime} \mathcal{N}(c)^{-s} \sum_{\substack{d\left(\Lambda_{\ell_{2}}\right) \\
d \equiv 1(3)}}^{(*)}\left(\frac{c}{d}\right)_{3}\right), \\
& \left\langle\theta_{\pi^{\ell_{1} \nu_{1}}}, \theta_{\pi^{\ell_{1} \nu_{1}}}^{\prime}\right\rangle=\operatorname{Res}_{s=4 / 3}\left(\sum_{c \equiv 1(3)}^{\prime} \mathcal{N}(c)^{-s} \sum_{\substack{d\left(\Lambda_{\ell_{1}} c\right) \\
d \equiv-1(3)}}^{(*)}\left(\frac{c}{d}\right)_{3}\right),
\end{aligned}
$$

where $(*)$ means

$$
c \not \equiv \alpha_{2} d,
$$

if $\ell_{1}=\ell_{2}=0$, and otherwise, i.e. $\left(\ell_{1} \ell_{2}\right) \neq(0,0),(*)$ means the conditions

$$
\operatorname{ord}_{\pi}(c) \geqslant \ell_{1} \quad \text { and } \quad d \alpha_{2} \nu_{2} \pi^{\ell_{2}-\ell_{1}}-\bar{d} \alpha_{1} \nu_{1} \equiv c \pi^{-\ell_{1}} \quad\left(\bmod \pi^{\min \left(\ell_{1}, h-\ell_{1}\right)}\right) .
$$

Moreover, the first condition is an equality, i.e. $\operatorname{ord}_{\pi}(c)=\ell_{1}$, in the case where $\ell_{1}<\ell_{2}$.

Proof. Let $\sigma^{-1}(\infty)$ and $\tau^{-1}(\infty)$ be two essential cusps. We start by proving that

$$
\bar{\kappa}\left(\sigma^{-1}\left(\begin{array}{ll}
a & b \\
c & d
\end{array}\right) \tau\right)=\left(\frac{c}{d}\right)_{3} .
$$


The computation of the Kubota symbol is easy, since one can assume (see Lemma 3.5) that $\sigma, \tau \in$ $\Gamma_{2}$. Moreover $\sigma^{-1}\left(\begin{array}{ll}a & b \\ c & d\end{array}\right) \tau \in \Gamma \subseteq \Gamma_{1} \subset \Gamma_{2}$, and since $\kappa$ is a group homomorphism on $\Gamma_{2}$, we have

$$
\kappa\left(\sigma^{-1}\left(\begin{array}{ll}
a & b \\
c & d
\end{array}\right) \tau\right)=\kappa\left(\sigma^{-1}\right) \kappa\left(\left(\begin{array}{ll}
a & b \\
c & d
\end{array}\right)\right) \kappa(\tau) .
$$

To show that $\kappa\left(\sigma^{-1}\right)=1$, we have to prove it for the four possible choice of $\sigma$, whenever a given couple $(\ell, \nu)$ is given. Write $\xi=\pi^{\ell} \nu$.

If $\sigma^{-1}=\left(\begin{array}{cc}1 & 0 \\ 3 \xi & 1\end{array}\right)$, then $\sigma^{-1} \in \Gamma_{1}$ and by definition of $\kappa$ on $\Gamma_{1}, \kappa\left(\sigma^{-1}\right)=\left(\frac{3 \xi}{1}\right)_{3}=1$.

If $\sigma^{-1}=\left(\begin{array}{cc}1 & 0 \\ \pm \xi & 1\end{array}\right)$, then $\sigma^{-1}=\left(\begin{array}{cc}1 & 0 \\ \pm & 1\end{array}\right)\left(\begin{array}{cc}1 & 0 \\ \pm \xi \mp 1 & 1\end{array}\right)$. The first matrix belongs to $\mathrm{SL}_{2}(\mathbb{Z})$ and the second to $\Gamma_{1}$, thus, by definition of $\kappa$ on $\Gamma_{2}, \kappa\left(\sigma^{-1}\right)=\kappa\left(\left(\begin{array}{cc}1 & 0 \\ \pm \xi \mp 1 & 1\end{array}\right)\right)=1, \xi$ being or not equal to 1 .

If finally, $\sigma^{-1}=\left(\begin{array}{cc}1 & -1 \\ 0 & 1\end{array}\right)\left(\begin{array}{ll}1 & 0 \\ \xi & 1\end{array}\right)=\left(\begin{array}{cc}0 & -1 \\ 1 & 1\end{array}\right)\left(\begin{array}{cc}\xi & 0 \\ \xi-1 & 1\end{array}\right)$, by definition of $\kappa$ on $\Gamma_{2}$, one obtains $\kappa\left(\sigma^{-1}\right)=1$. The same is true for $\kappa(\tau)$ and it remains to show that $\kappa\left(\left(\begin{array}{ll}a & b \\ c & d\end{array}\right)\right)=1$ under the conditions of the cases (i) and (ii) of the lemma. We have $\gamma \equiv \sigma \tau^{-1}(\bmod 3)$, with either $\sigma^{-1}(\infty)=\left(\alpha_{1} \pi^{\ell_{1}} \nu_{1}\right)^{-1}$ and $\tau^{-1}(\infty)=\left(\alpha_{2} \pi^{\ell_{2}} \nu_{2}\right)^{-1}$, in the case (i) of the lemma, or $\sigma^{-1}(\infty)=$ $\left(\pi^{\ell} \nu\right)^{-1}$ and $\tau^{-1}(\infty)=\left(\pi^{\ell} \nu\right)^{-1}-1$, in the case (ii) of the lemma. In case (i), $\gamma \equiv\left(\begin{array}{cc}1 & 0 \\ \alpha & 1\end{array}\right)$ $(\bmod 3)$, with $\alpha \in\{0, \pm 1\}$ and in case (ii), $\gamma \equiv\left(\begin{array}{ll}0 & -1 \\ 1 & -1\end{array}\right) \quad(\bmod 3)$. We recall that if $\gamma=\left(\begin{array}{ll}a & b \\ c & d\end{array}\right) \in$ $\Gamma_{2}$, then $\left(\frac{c}{a}\right)_{3}=\overline{\left(\frac{c}{d}\right)_{3}}$.

$$
\begin{aligned}
& \text { If }\left(\begin{array}{ll}
a & b \\
c & d
\end{array}\right) \equiv\left(\begin{array}{ll}
1 & 0 \\
0 & 1
\end{array}\right)(\bmod 3), \kappa\left(\left(\begin{array}{ll}
a & b \\
c & d
\end{array}\right)\right)=\left(\frac{c}{a}\right)_{3}=\overline{\left(\frac{c}{d}\right)_{3}} \text {. } \\
& \text { If }\left(\begin{array}{ll}
a & b \\
c & d
\end{array}\right) \equiv\left(\begin{array}{ll}
1 & 0 \\
\pm 1 & 1
\end{array}\right)(\bmod 3) \text {, } \\
& \kappa\left(\left(\begin{array}{ll}
a & b \\
c & d
\end{array}\right)\right)=\kappa\left(\left(\begin{array}{cc}
1 & 0 \\
\pm 1 & 1
\end{array}\right)\left(\begin{array}{cc}
1 & 0 \\
\mp 1 & 1
\end{array}\right)\left(\begin{array}{ll}
a & b \\
c & d
\end{array}\right)\right) \\
& =\kappa\left(\left(\begin{array}{cc}
a & b \\
c \mp a & d \mp b
\end{array}\right)\right)=\left(\frac{c \mp a}{a}\right)_{3}=\overline{\left(\frac{c}{d}\right)_{3}} . \\
& \text { If }\left(\begin{array}{ll}
a & b \\
c & d
\end{array}\right) \equiv\left(\begin{array}{ll}
0 & -1 \\
1 & -1
\end{array}\right) \quad(\bmod 3) \\
& \kappa\left(\left(\begin{array}{ll}
a & b \\
c & d
\end{array}\right)\right)=\kappa\left(\left(\begin{array}{ll}
0 & -1 \\
1 & -1
\end{array}\right)\left(\begin{array}{ll}
-1 & 1 \\
-1 & 0
\end{array}\right)\left(\begin{array}{ll}
a & b \\
c & d
\end{array}\right)\right) \\
& =\kappa\left(\left(\begin{array}{cc}
c-a & d-b \\
-a & -b
\end{array}\right)\right)=\left(\frac{-a}{c-a}\right)_{3}=\overline{\left(\frac{d}{c}\right)_{3}}=\overline{\left(\frac{c}{d}\right)_{3}} .
\end{aligned}
$$

This proves the result about the Kubota symbol. We now have to interpret the summation condition in (3.1.6).

Let $c \neq 0$, and $(d, c)=1$. Write $\beta_{i}=\alpha_{i} \nu_{i}$, for $i=1,2$ and look at the formula 3.1.6. In (i) the condition on $(c, d)$ is

$$
\left(\begin{array}{cc}
1 & 0 \\
\beta_{1} \pi^{\ell_{1}} & 1
\end{array}\right)\left(\begin{array}{ll}
a & b \\
c & d
\end{array}\right)\left(\begin{array}{cc}
1 & 0 \\
-\beta_{2} \pi^{\ell_{2}} & 1
\end{array}\right) \in \Gamma_{0}^{*}\left(\pi^{h}\right)
$$

i.e.

$$
\left(\begin{array}{cc}
a-b \beta_{2} \pi^{\ell_{2}} & b \\
c+a \beta_{1} \pi^{\ell_{1}}-d \beta_{2} \pi^{\ell_{2}}-b \beta_{1} \beta_{2} \pi^{\ell_{1}+\ell_{2}} & d+b \beta_{1} \pi^{\ell_{1}}
\end{array}\right) \in \Gamma_{0}^{*}\left(\pi^{h}\right),
$$

i.e.

$$
\left\{\begin{array}{rlr}
\left(\begin{array}{ll}
a & b \\
c & d
\end{array}\right) & \equiv\left(\begin{array}{cc}
1 & 0 \\
\alpha_{2}-\alpha_{1} & 1
\end{array}\right) & (\bmod 3) \\
c+a \beta_{1} \pi^{\ell_{1}}-d \beta_{2} \pi^{\ell_{2}}-b \beta_{1} \beta_{2} \pi^{\ell_{1}+\ell_{2}} & \equiv 0 & \left(\bmod \pi^{h}\right)
\end{array} .\right.
$$


Similarly, in (ii) the condition is

$$
\left(\begin{array}{cc}
1 & 0 \\
\beta_{1} \pi^{\ell_{1}} & 1
\end{array}\right)\left(\begin{array}{ll}
a & b \\
c & d
\end{array}\right)\left(\begin{array}{cc}
1 & 0 \\
-\beta_{1} \pi^{\ell_{1}} & 1
\end{array}\right)\left(\begin{array}{ll}
1 & 1 \\
0 & 1
\end{array}\right) \in \Gamma_{0}^{*}\left(\pi^{h}\right)
$$

i.e.

$$
\left(\begin{array}{cc}
a-b \beta_{1} \pi^{\ell_{1}} & b+a-b \beta_{1} \pi^{\ell_{1}} \\
c+a \beta_{1} \pi^{\ell_{1}}-d \beta_{1} \pi^{\ell_{1}}-b \beta_{1}^{2} \pi^{2 \ell_{1}} & d+b \beta_{1} \pi^{\ell_{1}}+c+a \beta_{1} \pi^{\ell_{1}}-d \beta_{1} \pi^{\ell_{1}}-b \beta_{1}^{2} \pi^{2 \ell_{1}}
\end{array}\right) \in \Gamma_{0}^{*}\left(\pi^{h}\right),
$$

i.e.

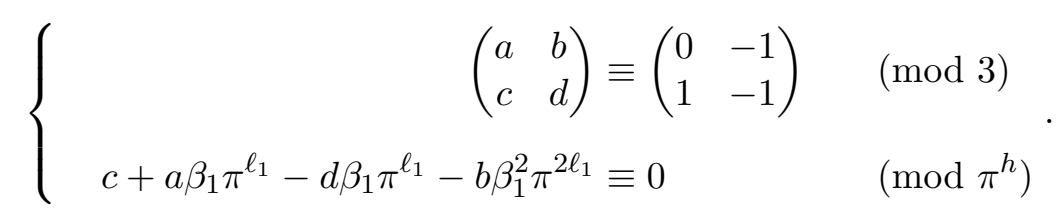

Thus $(c, d)$ has to satisfy $c \equiv \alpha_{2}-\alpha_{1}(\bmod 3)$ and $d \equiv 1(\bmod 3)$, in the case (i), or $c \equiv 1$ $(\bmod 3)$ and $d \equiv-1(\bmod 3)$, in the case (ii). Since $\operatorname{gcd}(\pi, 3)=1$, the chinese remainder theorem asserts that we only have to study the congruence condition modulo $\pi^{h}$. Let $a_{0}, b_{0}$ such that $a_{0} d-b_{0} c=1$. Then $(a, b)$ is of the form $\left(a_{0}+k c, b_{0}+k d\right)$, and we ask if there exists $k$ such that

$$
c+a_{0} \beta_{1} \pi^{\ell_{1}}-d \beta_{2} \pi^{\ell_{2}}-b_{0} \beta_{1} \beta_{2} \pi^{\ell_{1}+\ell_{2}}+k \beta_{1} \pi^{\ell_{1}}\left(c-d \beta_{2} \pi^{\ell_{2}}\right) \equiv 0 \quad\left(\bmod \pi^{h}\right) .
$$

This is the equation in case (ii), but for $\beta_{1}=\beta_{2}$ and $\ell_{1}=\ell_{2}$, this corresponds to case ( $i$ ).

If $\ell_{1}=\ell_{2}=0$, this equation has a solution if and only if $c-d \beta_{2} \not \equiv 0(\bmod \pi)$, i.e. $c \not \equiv \alpha_{2} d$ $(\bmod \pi)$, because $\left.\mathcal{P}_{\ell_{1}}=\mathcal{P}_{\ell_{2}}=\{1\}\right)$.

If $\left(\ell_{1}, \ell_{2}\right) \neq(0,0)$, we see from 3.2 .5$)$ that $c \equiv 0\left(\bmod \pi^{\ell_{1}}\right)$, and 3.2.5) becomes

$$
c \pi^{-\ell_{1}}+a_{0} \beta_{1}-d \beta_{2} \pi^{\ell_{2}-\ell_{1}}-b_{0} \beta_{1} \beta_{2} \pi^{\ell_{2}}+k \beta_{1} \pi^{\ell_{1}}\left(c \pi^{-\ell_{1}}-d \beta_{2} \pi^{\ell_{2}-\ell_{1}}\right) \equiv 0 \quad\left(\bmod \pi^{h-\ell_{1}}\right) .
$$

We remark that if $\ell_{1}<\ell_{2}$, then $c \pi^{-\ell_{1}}\left(1+k \beta_{1} \pi^{\ell_{1}}\right)+a_{0} \beta_{1} \equiv 0(\bmod \pi)$, what shows that $\operatorname{ord}_{\pi}(c)=\ell_{1}$, since otherwise $\pi \mid a_{0}$, what contradicts $a_{0} d-b_{0} c=1$.

Now (3.2.6) implies $c \pi^{-\ell_{1}}+a_{0} \beta_{1}-d \beta_{2} \pi^{\ell_{2}-\ell_{1}} \equiv 0\left(\bmod \pi^{\min \left(\ell_{1}, h-\ell_{1}\right)}\right)$, and we claim that this is sufficient to prove the existence of $k$. Actually, if $\ell_{1}<\ell_{2}$, then $\pi$ divides $d \beta_{2} \pi^{\ell_{2}-\ell_{1}}$ but not $c \pi^{-\ell_{1}}$, thus $c \pi^{-\ell_{1}}-d \beta_{2} \pi^{\ell_{2}-\ell_{1}}$ is invertible, and if $\ell_{1}=\ell_{2}>0$, then $\pi \mid c$, thus $a_{0} d \equiv 1(\bmod \pi)$ and $a_{0} \beta_{1} \not \equiv 0(\bmod \pi)$, and in view of $c \pi^{-\ell_{1}}+a_{0} \beta_{1}-d \beta_{2} \pi^{\ell_{2}-\ell_{1}} \equiv 0\left(\bmod \pi^{\min \left(\ell_{1}, h-\ell_{1}\right)}\right)$ we also get $c \pi^{-\ell_{1}}-d \beta_{2} \pi^{\ell_{2}-\ell_{1}} \not \equiv 0(\bmod \pi)$.

We are now interested in the Gauß sum relative to the integer $3 \pi^{\varepsilon} c$ with some extra congruence conditions.

Lemma 3.7. Let $c \in R$ and define $c^{\prime}=c \mathcal{N}(\lambda)^{-\operatorname{ord}_{\lambda}(c)} \pi^{-\operatorname{ord}_{\pi}(c)}$. Let $k, \varepsilon \in \mathbb{Z}$ with $k \geqslant 1$ and $\varepsilon \geqslant 0$. Let $\alpha \in\{0, \pm 1\}$, and let $v \in R$ be some integer.

If $c \equiv 0(\bmod 3)$ and $\alpha \neq 0$, then 
(i) $\sum_{\substack{d\left(3 \varepsilon^{\varepsilon} c\right) \\ d \equiv \alpha(3)}}\left(\frac{c}{d}\right)_{3}= \begin{cases}\varphi\left(c^{\prime}\right)\left(\frac{v}{\pi^{\text {ord } \pi}(c)}\right)_{3} \mathcal{N}(\pi)^{\max \left(\varepsilon+\operatorname{ord}_{\pi}(c)-k, 0\right)} \mathcal{N}(\lambda)^{\operatorname{ord}_{\lambda}(c)} & \text { if } c \pi^{-\operatorname{ord}_{\pi}(c)} \text { is a cube, } \\ 0 & \text { otherwise. }\end{cases}$ $d \equiv \alpha(3)$
$d \equiv v\left(\pi^{k}\right)$

(ii) $\sum_{\substack{d\left(3 \pi \varepsilon_{c}\right) \\ d \equiv \alpha(3)}}\left(\frac{c}{d}\right)_{3}= \begin{cases}\varphi\left(c^{\prime}\right) \mathcal{N}(\pi)^{\epsilon} \varphi\left(\pi^{\operatorname{ord}_{\pi}(c)}\right) \mathcal{N}(\lambda)^{\operatorname{ord}_{\lambda}(c)} & \text { if } c \text { is a cube } \\ 0 & \text { otherwise. }\end{cases}$

If $c \equiv \pm 1(\bmod 3)$, then

(iii) $\sum_{\substack{d\left(3 \pi^{\varepsilon} c \\ d \equiv \alpha(3) \\ d=\alpha(a)\right.}}\left(\frac{d}{c}\right)_{3}= \begin{cases}\varphi\left(c^{\prime}\right)\left(\frac{v}{\pi^{\text {ord } \pi(c)}}\right)_{3} \mathcal{N}(\pi)^{\max \left(\varepsilon+\operatorname{ord}_{\pi}(c)-k, 0\right)} & \text { if } c \pi^{-\operatorname{ord}_{\pi}(c)}= \pm c^{\prime} \text { is a cube, } \\ 0 & \text { otherwise. }\end{cases}$

(iv) $\sum_{\substack{d\left(3 \pi \varepsilon_{c}\right) \\ d \equiv \alpha(3)}}\left(\frac{d}{c}\right)_{3}= \begin{cases}\varphi\left(c^{\prime}\right) \mathcal{N}(\pi)^{\varepsilon} \varphi\left(\pi^{\mathrm{ord}_{\pi}(c)}\right) & \text { if } c \text { is a cube } \\ 0 & \text { otherwise. }\end{cases}$

Proof. We write $c=u \lambda^{i} \pi^{j} c^{\prime}$ with $u \in \mathcal{U}, \operatorname{gcd}\left(\pi \lambda, c^{\prime}\right)=1$ and $c^{\prime} \equiv 1(\bmod 3)$. We start by proving (i) and (ii). We introduce a parameter $k^{\prime} \geqslant 0$ which will be $k$ if $k^{\prime}>0$ and study the sum

$$
\sum_{\substack{d\left(\lambda^{2+i} \pi^{\varepsilon+j} \\ d \equiv \alpha(3) \\ d \equiv v\left(\pi^{\prime}\right)\right.}}\left(\frac{c}{d}\right)_{3}, \quad \text { for } \alpha= \pm 1 .
$$

Let $m, n$ such that $m \lambda^{2+i}+n \pi^{\varepsilon+j} c^{\prime}=1$. Then $d$ can be written as $d_{1} m \lambda^{2+i}+\alpha d_{2} n \pi^{\varepsilon+j} c^{\prime}$, where $d_{1}$ varies modulo $\pi^{\varepsilon+j} c^{\prime}$ and $d_{2}$ varies modulo $\lambda^{i+2}$. Remark that $n \equiv 1(\bmod 3)$, thus the condition $d \equiv \alpha(\bmod 3)$ is replaced by $d_{2} \equiv 1(\bmod 3)$. Likewise, $d \equiv v\left(\bmod \pi^{k^{\prime}}\right)$ becomes $d_{1} \equiv v\left(\bmod \pi^{\min \left(\varepsilon+j, k^{\prime}\right)}\right)$, i.e.

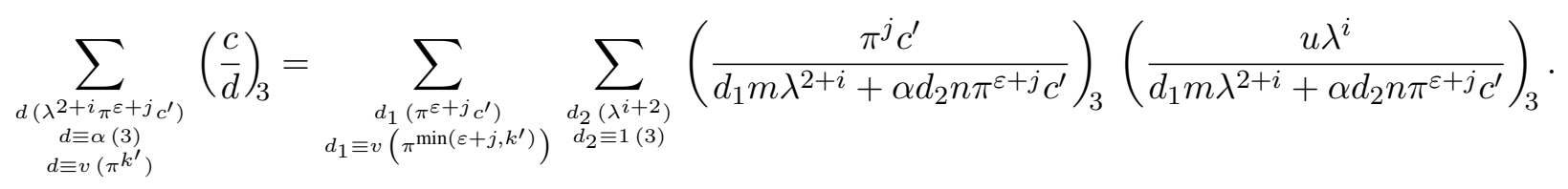

For the first factor, we use the reciprocity law, and for the second, the hypothesis $i \geqslant 2$ allows us to use the consequence of the complementary reciprocity law. We obtain that

$$
\begin{aligned}
\sum_{\substack{d\left(\lambda^{2+i} \pi^{\varepsilon+j} \\
d \equiv \alpha(3) \\
d \equiv v\left(\pi^{\prime}\right)\right.}}\left(\frac{c}{d}\right)_{3}= & \sum_{\substack{d_{1}\left(\pi^{\varepsilon+j} c^{\prime}\right) \\
d_{1} \equiv v\left(\pi^{\min \left(\varepsilon+j, k^{\prime}\right)}\right)}}\left(\frac{d_{1} m \lambda^{2+i}}{\pi^{j} c^{\prime}}\right)_{3} \sum_{\substack{d_{2}\left(\lambda^{i+2}\right) \\
d_{2} \equiv 1(3)}}\left(\frac{u \lambda^{i}}{\alpha d_{2} n \pi^{\varepsilon+j} c^{\prime}}\right)_{3} \\
& =\sum_{\substack{d\left(\pi^{\left.\varepsilon+j_{c^{\prime}}\right)} \\
d \equiv v\left(\pi^{\min \left(\varepsilon+j, k^{\prime}\right)}\right)\right.}}\left(\frac{d}{\pi^{j} c^{\prime}}\right)_{3} \times \sum_{\substack{d\left(\lambda^{i+2}\right) \\
d \equiv 1(3)}}\left(\frac{u \lambda^{i}}{d}\right)_{3} .
\end{aligned}
$$


It is easy to see that the second sum is $3^{i}$, if $i \equiv 0(\bmod 3)$ and $u= \pm 1$, and 0 otherwise. For the first sum, we use again the chinese remainder theorem. Let $m, n$ such that $m \pi^{\varepsilon+j}+n c^{\prime}=1$. We replace $d$ modulo $\pi^{\varepsilon+j} c^{\prime}$ by $d=d_{1} m \pi^{\varepsilon+j}+d_{2} n c^{\prime}$, where $d_{1}$ varies modulo $c^{\prime}$, and $d_{2}$ modulo $\pi^{\varepsilon+j}$. Then $\left(\frac{d}{\pi^{j} c^{\prime}}\right)_{3}=\left(\frac{d_{2}}{\pi^{j}}\right)_{3}\left(\frac{d_{1}}{c^{\prime}}\right)_{3}$ and

$$
\sum_{\substack{d\left(\pi^{\left.\varepsilon+j^{\prime}\right)} \\ d \equiv v\left(\pi^{\min \left(\varepsilon+j, k^{\prime}\right)}\right)\right.}}\left(\frac{d}{\pi^{j} c^{\prime}}\right)_{3}=\sum_{d_{1}\left(c^{\prime}\right)}\left(\frac{d_{1}}{c^{\prime}}\right)_{3} \sum_{\substack{d_{2}\left(\pi^{\varepsilon}+j\right) \\ d_{2} \equiv v\left(\pi^{\min \left(\varepsilon+j, k^{\prime}\right)}\right)}}\left(\frac{d_{2}}{\pi^{j}}\right)_{3} .
$$

The first sum is equal to $\varphi\left(c^{\prime}\right)$ if $c^{\prime}$ is a cube and to 0 otherwise. The second sum depends on $k^{\prime}$. If $k^{\prime}=0$, i.e. in the case $(i i)$, it is $\varphi\left(\pi^{\varepsilon+j}\right)$ if $j \equiv 0(\bmod 3)$ and 0 otherwise. If $k^{\prime}>0$, i.e. in the case (i) with $k^{\prime}=k$, it is $\left(\frac{v}{\pi^{j}}\right) \mathcal{N}(\pi)^{\max (\varepsilon+j-k, 0)}$.

Proof of (iii) and (iv). With the same choice of $k$ as above, we have, for $\alpha \in\{0, \pm 1\}$,

$$
\sum_{\substack{d \lambda^{2} \pi^{\varepsilon+j} \\ d \equiv \alpha(3) \\ d \equiv v\left(\pi^{\prime}\right)}}\left(\frac{d}{c}\right)_{3}=\sum_{\substack{d\left(\pi^{\varepsilon+j} j^{\prime}\right) \\ \alpha+3 d \equiv v\left(\pi^{\min \left(\varepsilon+j, k^{\prime}\right)}\right)}}\left(\frac{\alpha+3 d}{\pi^{j} c^{\prime}}\right)_{3} .
$$

As above, let $m, n$, such that $m \pi^{\varepsilon+j}+n c^{\prime}=1$ and replace $d$ by $d_{1} m \pi^{\varepsilon+j}+d_{2} n c^{\prime}$, with $d_{1}$ modulo $c^{\prime}$ and $d_{2}$ modulo $\pi^{\varepsilon+j}$. Then

$$
\begin{aligned}
\sum_{\substack{d\left(\lambda^{2} \pi^{\varepsilon+j} c^{\prime}\right) \\
d \equiv \alpha(3) \\
d \equiv v\left(\pi^{\prime}\right)}}\left(\frac{d}{c}\right)_{3} & =\sum_{d_{1}\left(c^{\prime}\right)} \sum_{\substack{\alpha+3 d_{2} \equiv v\left(\pi^{\min \left(\varepsilon+j, k^{\prime}\right)}\right) \\
d_{2}\left(\pi^{\prime}\right)+j}}\left(\frac{\alpha+3 d_{1} m \pi^{\varepsilon+j}}{c^{\prime}}\right)_{3}\left(\frac{\alpha+3 d_{2} n c^{\prime}}{\pi^{j}}\right)_{3} \\
& =\sum_{d_{1}\left(c^{\prime}\right)}\left(\frac{d_{1}}{c^{\prime}}\right)_{3} \times \sum_{\substack{d_{2}\left(\pi^{\varepsilon+j}\right) \\
d_{2} \equiv v\left(\pi^{\min \left(\varepsilon+j, k^{\prime}\right)}\right)}}\left(\frac{d_{2}}{\pi^{j}}\right)_{3} .
\end{aligned}
$$

We conclude the proof of (iii) and (iv) as we concluded the proof of (i) and (ii).

Let $f \in R$ be an integer and let $\chi$ be a primitive character modulo $f$. The Dirichlet series associated to $\chi$ is

$$
L(\chi, s)=\prod_{\pi \chi_{f}}\left(1-\chi(\pi) \mathcal{N}(\pi)^{-s}\right)^{-1} .
$$

This series has no pole in $\Re(s)>0$ if $\chi$ is not trivial. If $\chi=1$, then $(\chi, s)=\zeta_{k}(s)$ has a pole at $s=1$ with residue $\alpha$.

Lemma 3.8. Let $\chi$ be a character with conductor $f$, let $g \in R$ and let $n \in \mathbb{Z}, n \geq 1$. Then

$$
\sum_{\substack{c \equiv 1(3) \\ \operatorname{gcd}(c, f g)=1}} \chi\left(c^{n}\right) \mathcal{N}\left(c^{n}\right)^{-s} \varphi\left(c^{n}\right)=\frac{L\left(\chi^{n}, n s-n\right)}{L\left(\chi^{n}, n s-n+1\right)} \prod_{\pi \mid g}\left\{\frac{1-\chi^{n}(\pi) \mathcal{N}(\pi)^{n-n s)}}{1-\chi^{n}(\pi) \mathcal{N}(\pi)^{n-1-n s)}}\right\} .
$$

In particular,

$$
\operatorname{Res}_{s=1+1 / n}\left(\sum_{\substack{c \equiv=1(3) \\ \operatorname{gcd}(c, f g)=1}} \chi\left(c^{n}\right) \mathcal{N}\left(c^{n}\right)^{-s} \varphi\left(c^{n}\right)\right)= \begin{cases}\frac{\alpha}{n \zeta_{k}(2)} \prod_{\pi \mid f g} \frac{\mathcal{N}(\pi)}{\mathcal{N}(\pi)+1} & \text { if } \chi^{n}=1, \\ 0 & \text { otherwise }\end{cases}
$$


Proof. Since we take the sum over the $c \equiv 1(\bmod 3)$, we can write them as $c=\sum_{m} \prod_{\pi} \pi^{m}$, $\pi \neq \lambda, \pi$ being primary, i.e. $\pi \equiv 1(\bmod 3)$. Then

$$
\begin{aligned}
& \sum_{\substack{c \equiv 1(3) \\
\operatorname{gcd}(c, f g)=1}} \chi\left(c^{n}\right) \mathcal{N}\left(c^{n}\right)^{-s} \varphi\left(c^{n}\right)=\prod_{\pi \chi_{f g}} \sum_{m \geq 0} \chi\left(\pi^{n m}\right) \mathcal{N}(\pi)^{-n m s} \varphi\left(\pi^{n m}\right) \\
= & \prod_{\pi \chi_{f g}}\left\{1+\frac{(\mathcal{N}(\pi)-1)}{\mathcal{N}(\pi)} \sum_{m \geq 1} \chi\left(\pi^{n m}\right) \mathcal{N}(\pi)^{n m(1-s)}\right\}=\prod_{\pi \chi_{f g}}\left\{\frac{1-\chi^{n}(\pi) \mathcal{N}(\pi)^{n-1-n s}}{1-\chi^{n}(\pi) \mathcal{N}(\pi)^{n(1-s)}}\right\} \\
= & L\left(\chi^{n}, n s-n\right) L\left(\chi^{n}, n s-n+1\right)^{-1} \prod_{\pi \mid g}\left\{\frac{1-\chi^{n}(\pi) \mathcal{N}(\pi)^{n-n s}}{1-\chi^{n}(\pi) \mathcal{N}(\pi)^{n-1-n s}}\right\} .
\end{aligned}
$$

If $\chi^{n}=1$, then

$$
\begin{aligned}
& \operatorname{Res}_{s=1+1 / n}\left(L\left(\chi^{n}, n s-n\right) L\left(\chi^{n}, n s-n+1\right)^{-1} \prod_{\pi \mid g}\left\{\frac{1-\chi^{n}(\pi) \mathcal{N}(\pi)^{n-n s}}{1-\chi^{n}(\pi) \mathcal{N}(\pi)^{n-1-n s}}\right\}\right) \\
& =\operatorname{Res}_{s=1+1 / n}\left(\prod_{\pi \chi_{f}} \frac{1-\mathcal{N}(\pi)^{n-1-n s}}{1-\mathcal{N}(\pi)^{n-n s}} \prod_{\pi \mid g}\left\{\frac{1-\mathcal{N}(\pi)^{n-n s}}{1-\mathcal{N}(\pi)^{n-1-n s}}\right\}\right) \\
& =\operatorname{Res}_{s=1+1 / n}\left(\frac{\zeta_{k}(n s-n)}{\zeta_{k}(n s-n+1)} \prod_{\pi \mid f g}\left\{\frac{1-\mathcal{N}(\pi)^{n-n s}}{1-\mathcal{N}(\pi)^{n-1-n s}}\right\}\right)=\frac{\alpha}{n \zeta_{k}(2)} \prod_{\pi \mid \mathfrak{f g}} \frac{\mathcal{N}(\pi)}{\mathcal{N}(\pi)+1}
\end{aligned}
$$

Lemma 3.9. Let $\beta \in\{0, \pm 1\}$. Then

$$
\sum_{u= \pm 1} \sum_{\substack{i \geq 0 \\ u \lambda \\ u i \equiv \beta(3)}} \mathcal{N}(\lambda)^{-i}=1 .
$$

Proof. If $\beta=0$, the left hand side is equal to

$$
\sum_{u= \pm 1} \sum_{i \geq 1} \mathcal{N}(\lambda)^{-i}=2\left(\sum_{i \geq 0} \frac{1}{3^{i}}-1\right)=1
$$

and if $\beta= \pm 1$, it is equal to

$$
\sum_{u=\beta} \sum_{i=0} \mathcal{N}(\lambda)^{-i}=1
$$

and the lemma is proved.

\subsubsection{Scalar product of theta functions}

As in Section 3.2.1, we still work with the group $\Gamma_{0}^{*}\left(\pi^{h}\right)$ for a fixed integer $h \geqslant 1$. Recall that to any given parameter $\xi=(\ell, \nu)$ with $0 \leqslant \ell \leqslant h$ and $\nu \in \mathcal{P}_{\ell}$ (defined in (3.2.1)) correspond the four theta functions

$$
\theta_{\xi}, \theta_{-\xi}, \theta_{3 \xi} \text { and } \theta_{\xi}^{\prime} \text {, }
$$


which were defined in (3.2.3) and (3.2.4). The lattice corresponding to any of these four functions only depends on $\ell$; we write it $\Lambda_{\ell}$, and recall that $\Lambda_{\ell}=3 \pi^{\operatorname{Max}(0, h-2 \ell)} R$. In relation to this, we define

$$
M(\ell)=\operatorname{Max}(0, h-2 \ell) \quad \text { and } \quad m(\ell)=\min (\ell, h-\ell) .
$$

We are now able to work out the scalar product of two theta functions, from the general formula (3.1.6.).

We shall deal with the following exponential sums:

$$
s(v)=\sum_{x(\pi)}^{\times}\left(\frac{x^{2} v-1}{\pi}\right)_{3} \quad \text { and } \quad t(v)=\sum_{x(\pi)}^{\times}\left(\frac{x v-\bar{x}}{\pi}\right)_{3} .
$$

Then, defining the characters $\chi_{2}=(\dot{\bar{\pi}})_{2}$ and $\chi_{3}=(\dot{\bar{\pi}})_{3}$, one has

$$
\begin{aligned}
& s(v)=\left(\frac{v}{\pi}\right)_{2}, J\left(\chi_{3}, \chi_{2}\right)-1, \\
& t(v)=\left(\frac{v}{\pi}\right)_{3}^{2} J\left(\chi_{3}, \chi_{3}\right)+\left(\frac{v}{\pi}\right)_{3}^{2}\left(\frac{v}{\pi}\right)_{2} J\left(\chi_{3}, \chi_{3} \chi_{2}\right),
\end{aligned}
$$

where $J(\chi, \psi)$ is the Jacobi sum

$$
J(\chi, \psi)=\sum_{x(\pi)} \chi(x) \psi(x-1)
$$

Proposition 3.3. Let $\xi=(\ell, \nu)$ be given. Then the functions $\theta_{\xi}$ and $\theta_{\xi}^{\prime}$ are equal. Moreover, if $m=m(\ell)$ and $M=M(\ell)$ are defined by (3.2.7), then

$$
\begin{aligned}
\left\|\theta_{\xi}\right\|^{2}= & \frac{\alpha}{3 \zeta_{k}(2)} \frac{\mathcal{N}(\pi)^{h+1}}{\mathcal{N}(\pi)+1} & \text { if } \ell=0, \\
\left\|\theta_{\xi}\right\|^{2}= & \frac{2 \alpha}{3 \zeta_{k}(2)} \frac{\mathcal{N}(\pi)^{M-m-\ell / 3+1}}{\mathcal{N}(\pi)^{2}-1}\left(\frac{\mathcal{N}(\pi)-\mathcal{N}(\pi)^{(1-m) / 3}}{\mathcal{N}(\pi)^{1 / 3}-1}\right. & \text { if } 1 \leqslant \ell \leqslant h-1, \\
& +\left\{\begin{array}{lll}
\Re\left(\left(\frac{\nu}{\pi}\right)_{3} t(1)\right) & \text { if } \ell \equiv 0 & (\bmod 3) \\
\frac{1}{2}(\mathcal{N}(\pi)-1)+\Re\left(\left(\frac{\nu}{\pi}\right)_{3} s(1)\right) & \text { if } \ell \neq \equiv 0 & (\bmod 3)
\end{array}\right) & \\
\left\|\theta_{\xi}\right\|^{2}= & \frac{\alpha}{3 \zeta_{k}(2)} \frac{\mathcal{N}(\pi)^{1-\lceil h / 3\rceil}}{\mathcal{N}(\pi)+1} & \text { if } \ell=h .
\end{aligned}
$$

Proof. Denote simply by $\Lambda$ the lattice $\Lambda_{\ell}$ associated to any of the four theta functions correspond- 
ing to $\xi$. The formula (3.1.6) of Section 3.1.2 gives then

$$
\begin{aligned}
& \left\langle\theta_{\xi}, \theta_{\xi}\right\rangle=\operatorname{Res} \sum_{c}^{\prime} \mathcal{N}(c)^{-s} \sum_{d(\Lambda c)} \bar{\kappa}\left(\left(\begin{array}{ll}
1 & 0 \\
\xi & 1
\end{array}\right)\left(\begin{array}{ll}
a & b \\
c & d
\end{array}\right)\left(\begin{array}{cc}
1 & 0 \\
-\xi & 1
\end{array}\right)\right), \\
& \left(\begin{array}{ll}
1 & 0 \\
\xi & 1
\end{array}\right)\left(\begin{array}{ll}
a & b \\
c & d
\end{array}\right)\left(\begin{array}{cc}
1 & 0 \\
-\xi & 1
\end{array}\right) \in \Gamma \\
& \left\langle\theta_{\xi}, \theta_{\xi}^{\prime}\right\rangle=\operatorname{Res} \sum_{c}^{\prime} \mathcal{N}(c)^{-s} \sum_{d(\Lambda c)} \bar{\kappa}\left(\left(\begin{array}{ll}
1 & 0 \\
\xi & 1
\end{array}\right)\left(\begin{array}{ll}
a & b \\
c & d
\end{array}\right)\left(\begin{array}{cc}
1 & 0 \\
-\xi & 1
\end{array}\right)\left(\begin{array}{ll}
1 & 1 \\
0 & 1
\end{array}\right)\right), \\
& \left(\begin{array}{ll}
1 & 0 \\
\xi & 1
\end{array}\right)\left(\begin{array}{ll}
a & b \\
c & d
\end{array}\right)\left(\begin{array}{cc}
1 & 0 \\
-\xi & 1
\end{array}\right)\left(\begin{array}{ll}
1 & 1 \\
0 & 1
\end{array}\right) \in \Gamma \\
& \left\langle\theta_{\xi}^{\prime}, \theta_{\xi}^{\prime}\right\rangle=\operatorname{Res} \sum_{c}^{\prime} \mathcal{N}(c)^{-s} \quad \sum_{d(\Lambda c)} \quad \bar{\kappa}\left(\left(\begin{array}{cc}
1 & -1 \\
0 & 1
\end{array}\right)\left(\begin{array}{ll}
1 & 0 \\
\xi & 1
\end{array}\right)\left(\begin{array}{ll}
a & b \\
c & d
\end{array}\right)\left(\begin{array}{cc}
1 & 0 \\
-\xi & 1
\end{array}\right)\left(\begin{array}{ll}
1 & 1 \\
0 & 1
\end{array}\right)\right) . \\
& \left(\begin{array}{cc}
1 & -1 \\
0 & 1
\end{array}\right)\left(\begin{array}{ll}
1 & 0 \\
\xi & 1
\end{array}\right)\left(\begin{array}{ll}
a & b \\
c & d
\end{array}\right)\left(\begin{array}{cc}
1 & 0 \\
-\xi & 1
\end{array}\right)\left(\begin{array}{ll}
1 & 1 \\
0 & 1
\end{array}\right) \in \Gamma
\end{aligned}
$$

Recall that $T=\left(\begin{array}{ll}1 & 1 \\ 0 & 1\end{array}\right)$. Since $T \Gamma T^{-1}=\Gamma$, we already have $\left\|\theta_{\xi}\right\|=\left\|\theta_{\xi}^{\prime}\right\|$. It remains to show that $\theta_{\xi}$ and $\theta_{\xi}^{\prime}$ are proportional, what we shall prove by the Cauchy-Schwarz criterion, by proving that $\left\langle\theta_{\xi}, \theta_{\xi}\right\rangle=\left\langle\theta_{\xi}, \theta_{\xi}^{\prime}\right\rangle$, i.e. by proving that 3.2.10 $=3.2 .11$. By Lemma 3.7, this equality is equivalent to the equality

$$
\operatorname{Res} \sum_{\substack{c \\ c \equiv 0(3)}}^{\prime} \mathcal{N}\left((c)^{-s} \sum_{d(\Lambda c)}^{*}\left(\frac{c}{d}\right)_{3}=\operatorname{Res} \sum_{\substack{c \\ c \equiv 1}}^{\prime} \mathcal{N}\left((c)^{-s} \sum_{d(\Lambda c)}^{*}\left(\frac{c}{d}\right)_{3},\right.\right.
$$

where $(*)$, as in Lemma 3.6, means that

- $c \not \equiv d(\bmod \pi)$, if $\ell=0$,

- $c \equiv 0\left(\bmod \pi^{\ell}\right)$ and $c \pi^{-\ell}-d \nu \equiv-\nu a_{0}\left(\bmod \pi^{\min (\ell, h-\ell)}\right)$, if $1 \leqslant \ell \leqslant h$.

We start with the case $\ell=0$. We have to prove that

$$
\operatorname{Res}_{s=4 / 3}\left(\sum_{c \equiv 0(3)}^{\prime} \mathcal{N}(c)^{-s} \sum_{\begin{array}{l}
d\left(3 \pi h_{c}\right) \\
d \equiv 1(3) \\
d \neq c(\pi)
\end{array}}\left(\frac{c}{d}\right)_{3}\right)=\operatorname{Res}_{s=4 / 3}\left(\sum_{c \equiv 1(3)}^{\prime} \mathcal{N}(c)^{-s} \sum_{\begin{array}{c}
d\left(3 \pi^{h}\right) \\
d \equiv-1(3) \\
d \neq c(\pi)
\end{array}}\left(\frac{c}{d}\right)_{3}\right) .
$$

For a given $c \equiv 1(\bmod 3)$ we have

$$
\sum_{\substack{d\left(3 h_{c}\right) \\
d \equiv-1(3) \\
d \neq c(\pi)}}\left(\frac{c}{d}\right)_{3}=\sum_{\substack{d\left(3 \pi h_{c}\right) \\
d \equiv-1(3)}}\left(\frac{c}{d}\right)_{3}-\sum_{\begin{array}{c}
d\left(3 \pi h_{c}\right) \\
d \equiv-1(3) \\
d \equiv c(\pi)
\end{array}}\left(\frac{c}{d}\right)_{3}
$$

Write $c=\pi^{j} c^{\prime}$ with $\operatorname{gcd}\left(c^{\prime}, \pi\right)=1$. Then, according to Lemma 3.7 about Gauß sums, this expression is not 0 only if $c^{\prime}$ is a cube, in which case we obtain

$$
\varphi\left(c^{\prime}\right)\left(\delta_{j \equiv 0(3)} \mathcal{N}(\pi)^{h} \varphi\left(\pi^{j}\right)-\left(\frac{c}{\pi^{j}}\right)_{3} \mathcal{N}(\pi)^{h+j-1}\right) .
$$

Remark that $j>0$ means $\pi \mid c$, and then the second term vanishes. Thus if $c^{\prime}$ is a cube, 


$$
\begin{aligned}
\sum_{\substack{d\left(3 \pi^{h}\right) \\
d \equiv=1(3) \\
d \neq c(\pi)}}\left(\frac{c}{d}\right)_{3} & =\varphi\left(c^{\prime}\right) \begin{cases}\delta_{j \equiv 0(3)} \mathcal{N}(\pi)^{h} \varphi\left(\pi^{j}\right) & \text { if } j>0 \\
\mathcal{N}(\pi)^{h}-\mathcal{N}(\pi)^{h-1} & \text { if } j=0\end{cases} \\
& =\varphi\left(c^{\prime}\right) \begin{cases}\mathcal{N}(\pi)^{h+j-1}(\mathcal{N}(\pi)-1) & \text { if } j \equiv 0 \quad(\bmod 3), \\
0 & \text { otherwise. }\end{cases}
\end{aligned}
$$

The same argument holds for a $c \equiv 0(\bmod 3)$. We deduce that the equality $(3.2 .13)$ is equivalent to

$$
\begin{aligned}
& \operatorname{Res}_{s=4 / 3}\left(\sum_{u \in\{ \pm 1\}} \sum_{i \geqslant 1} \sum_{j \geqslant 0} \sum_{c \equiv 1(3)}^{\prime} \mathcal{N}\left(u \lambda^{3 i} \pi^{3 j} c^{3}\right)^{-s} \varphi\left(c^{3}\right) \mathcal{N}(\lambda)^{3 i} \mathcal{N}(\pi)^{h+3 j-1}(\mathcal{N}(\pi)-1)\right) \\
= & \operatorname{Res}_{s=4 / 3}\left(\sum_{j \geqslant 0} \sum_{c \equiv 1(3)}^{\prime} \mathcal{N}\left(\pi^{3 j} c^{3}\right)^{-s} \varphi\left(c^{3}\right) \mathcal{N}(\pi)^{h+3 j-1}(\mathcal{N}(\pi)-1)\right) .
\end{aligned}
$$

That this equality holds comes from Lemma3.9. In particular, we proceed with the evaluation of the right side of the last equation, and we obtain that

$$
\begin{aligned}
\left\langle\theta_{\xi}, \theta_{\xi}\right\rangle & =\operatorname{Res}_{s=4 / 3}\left(\mathcal{N}(\pi)^{h-1}(\mathcal{N}(\pi)-1) \sum_{j \geq 1} \sum_{\substack{c \equiv 1(3) \\
(c, \pi)=1}} \mathcal{N}\left(c^{3} \pi^{3 j}\right)^{-s} \varphi\left(c^{3}\right) \mathcal{N}(\pi)^{3 j}\right) \\
& =\mathcal{N}(\pi)^{h-1} \operatorname{Res}_{s=4 / 3}\left(\sum_{\substack{c \equiv 1(3) \\
(c, \pi)=1}} \mathcal{N}\left(c^{3}\right)^{-s} \varphi\left(c^{3}\right)\right)\left((\mathcal{N}(\pi)-1) \sum_{j \geq 0} \mathcal{N}(\pi)^{-j}\right) .
\end{aligned}
$$

Lemma 3.8 finally gives

$$
\left\langle\theta_{\xi}, \theta_{\xi}\right\rangle=\frac{\alpha}{3 \zeta_{k}(2)} \frac{\mathcal{N}(\pi)^{h+1}}{\mathcal{N}(\pi)+1} .
$$

This concludes the proof of Proposition 3.3 in the case $\ell=0$.

For the case $\ell \neq 0$, we have seen in $(3.2 .13)$ that the following equality has to be proven:

$$
\operatorname{Res} \sum_{\substack{c \\ c \equiv 0(3)}}^{\prime} \mathcal{N}\left((c)^{-s} \sum_{d(\Lambda c)}^{*}\left(\frac{c}{d}\right)_{3}=\operatorname{Res} \sum_{\substack{c \\ c \equiv 1}}^{\prime} \mathcal{N}\left((c)^{-s} \sum_{d(\Lambda c)}^{*}\left(\frac{c}{d}\right)_{3},\right.\right.
$$

where $(*)$ means that $c \equiv 0\left(\bmod \pi^{\ell}\right)$ and $c \pi^{-\ell}-d \nu \equiv-\nu a_{0}\left(\bmod \pi^{\min (\ell, h-\ell)}\right)$. We replace the last condition by introducing the sum over the $x$ in $\left(R / \pi^{m} R\right)^{\times}$satisfying the same condition, and then summing over the $d$ 's congruent to $x$ modulo $\pi^{m}$. Thus, our claim is that the following equality holds:

$$
\operatorname{Res}_{s=4 / 3}\left(\sum_{x\left(\pi^{m}\right)}^{\times} \sum_{\substack{c \equiv 0(3) \\ c \equiv 0\left(\pi^{\ell}\right) \\ \frac{c}{\pi^{\ell}} \equiv x \nu-\bar{x} \nu\left(\pi^{m}\right)}}^{\prime} \mathcal{N}(c)^{-s} \sum_{\substack{d\left(3 \pi^{M} c\right) \\ d \equiv 1(3) \\ d \equiv x\left(\pi^{m}\right)}}\left(\frac{c}{d}\right)_{3}\right)=\operatorname{Res}_{s=4 / 3}\left(\sum_{x\left(\pi^{m}\right)}^{\times} \sum_{\substack{c \equiv 1(3) \\ c \equiv 0\left(\pi^{\ell}\right) \\ \frac{c}{\pi^{\ell}} \equiv x \nu-\bar{x} \nu\left(\pi^{m}\right)}}^{\prime} \mathcal{N}(c)^{-s} \sum_{\substack{d\left(3 M^{M} c\right) \\ d \equiv-1(3) \\ d \equiv x\left(\pi^{m}\right)}}\left(\frac{c}{d}\right)_{3}\right) .
$$


Lemma 3.7 about Gauß sums requires to know if $m=\min (\ell, h-\ell)=0$ or not. Since we assume $\ell \neq 0$, the only possibility is $\ell=h$, therefore we shall treat both cases separately. Notice that $\operatorname{ord}_{\pi}(c)+\max (0, h-2 \ell) \geqslant \ell+\max (\ell, h-\ell)-\ell \geqslant \min (\ell, h-\ell)$. Let us first assume $1 \leqslant \ell \leqslant h-1$. Then by Lemma 3.8, 3.2.15) is equivalent to

$$
\begin{array}{r}
\operatorname{Res}_{s=4 / 3}\left(\sum_{x\left(\pi^{m}\right)}^{\times} \sum_{u \in\{ \pm 1\}} \sum_{i \geqslant 1} \sum_{j \geqslant \ell} \sum_{\substack{c \equiv 1(3) \\
u \lambda^{3 i} \pi^{j-\ell} c^{3} \equiv x \nu-\bar{x} \nu\left(\pi^{m}\right)}} \mathcal{N}\left(u \lambda^{3 i} \pi^{j} c^{3}\right)^{-s} \varphi\left(c^{3}\right)\left(\frac{x}{\pi^{j}}\right)_{3} \mathcal{N}(\pi)^{M+j-m} \mathcal{N}(\lambda)^{3 i}\right) \\
=\operatorname{Res}_{s=4 / 3}\left(\sum_{x\left(\pi^{m}\right)}^{\times} \sum_{j \geqslant \ell} \sum_{\substack{c \equiv \equiv(3) \\
\pi^{j-\ell} c^{3} \equiv x \nu-\bar{x} \nu\left(\pi^{m}\right)}} \mathcal{N}\left(\pi^{j} c^{3}\right)^{-s} \varphi\left(c^{3}\right)\left(\frac{x}{\pi^{j}}\right)_{3} \mathcal{N}(\pi)^{M+j-m}\right) .
\end{array}
$$

This equality holds, by Lemma 3.9, and we now compute the norm of $\theta_{\xi}$. Remark that for $j$ big enough, i.e. $j \geqslant \ell+m$, then the condition $\pi^{j-\ell} c^{3} \equiv x \nu-\bar{x} \nu\left(\bmod \pi^{m}\right)$ becomes $x^{2} \equiv 1$ $\left(\bmod \pi^{m}\right)$, but for the remaining $j$ 's, i.e. $\ell \leqslant j \leqslant \ell+m-1$, it becomes $x^{2} \equiv 1\left(\bmod \pi^{j-\ell}\right)$ and $c^{3} \equiv \nu(x-\bar{x}) \pi^{-(j-\ell)}\left(\bmod \pi^{m-(j-\ell)}\right)$. This leads to

$$
\begin{array}{r}
\left\langle\theta_{\xi}, \theta_{\xi}\right\rangle=\operatorname{Res}_{s=4 / 3}\left(\sum_{j=\ell}^{\ell+m-1} \sum_{\substack{x\left(\pi^{m}\right) \\
x^{2} \equiv 1\left(\pi^{j}-\ell\right)}}^{\times} \sum_{\substack{c \equiv 1(3), \operatorname{gcd}(c, \pi)=1 \\
c^{3} \equiv x \nu-\bar{x} \nu\left(\pi^{m-(j-\ell)}\right)}} \mathcal{N}\left(\pi^{j} c^{3}\right)^{-s} \varphi\left(c^{3}\right)\left(\frac{x}{\pi^{j}}\right)_{3} \mathcal{N}(\pi)^{M+j-m}\right. \\
\left.\quad+\sum_{j \geqslant \ell+m} \sum_{\substack{x\left(\pi^{m}\right) \\
x^{2} \equiv 1\left(\pi^{m}\right)}}^{\times} \sum_{\substack{c \equiv 1(3) \\
(c, \pi)=1)}} \mathcal{N}\left(\pi^{j} c^{3}\right)^{-s} \varphi\left(c^{3}\right)\left(\frac{x}{\pi^{j}}\right)_{3} \mathcal{N}(\pi)^{M+j-m}\right) .
\end{array}
$$

Notice that the fact that we assume $\ell \neq h$ ensures $m=\min (\ell, h-\ell) \geqslant 1$, so that the first sum is not empty. To evaluate the inner sum of the first term, we remove the congruence condition introducing a character sum. More precisely, we have

$$
\begin{aligned}
\sum_{\substack{x\left(\pi^{m}\right) \\
x^{2} \equiv\left(\pi^{j-\ell}\right)}}^{\times} \sum_{\substack{c \equiv 1(3), \operatorname{gcd}(c, \pi)=1 \\
c^{3} \equiv x \nu-\bar{x} \nu\left(\pi^{m-(j-\ell)}\right)}} \mathcal{N}\left(\pi^{j} c^{3}\right)^{-s} \varphi\left(c^{3}\right)\left(\frac{x}{\pi^{j}}\right)_{3} \\
=\varphi\left(\pi^{m-(j-\ell)}\right)^{-1} \sum_{\substack{x\left(\pi^{m}\right) \\
x^{2} \equiv 1\left(\pi^{j-\ell}\right)}}^{\times} \sum_{\left(\pi^{m-(j-\ell)}\right)} \sum_{\substack{c \equiv 1(3) \\
\operatorname{gcd}(c, \pi)=1}} \chi\left((x-\bar{x}) \nu \bar{c}^{3}\right) \mathcal{N}\left(\pi^{j} c^{3}\right)^{-s} \varphi\left(c^{3}\right)\left(\frac{x}{\pi^{j}}\right)_{3} .
\end{aligned}
$$

In applying Lemma 3.9. we see that, taking the residue at $s=\frac{4}{3}$, only cubic characters will give a non trivial contribution. We thus obtain

$$
\begin{aligned}
\left\langle\theta_{\xi}, \theta_{\xi}\right\rangle= & \frac{\alpha}{3 \zeta_{k}(2)} \frac{\mathcal{N}(\pi)}{\mathcal{N}(\pi)+1} \mathcal{N}(\pi)^{M-m}\left(\sum_{j \geqslant \ell+m} \sum_{\substack{x\left(\pi^{m}\right) \\
x^{2} \equiv 1\left(\pi^{m}\right)}}^{\times} \mathcal{N}(\pi)^{-j / 3}\left(\frac{x}{\pi^{j}}\right)_{3}\right. \\
& \left.+\sum_{j=\ell}^{\ell+m-1} \varphi\left(\pi^{m-(j-l)}\right)^{-1} \sum_{\substack{x\left(\pi^{m}\right) \\
x^{2} \equiv\left(\pi^{j-\ell}\right)}}^{\times} \sum_{\substack{\left(\pi^{m-(j-\ell)}\right) \\
\chi^{3}=1}} \chi(\nu(x-\bar{x})) \mathcal{N}(\pi)^{-j / 3}\left(\frac{x}{\pi^{j}}\right)_{3}\right) .
\end{aligned}
$$


The only cubic characters modulo $\pi^{m-(j-\ell)}$ are the powers of the cubic Legendre symbol modulo $\pi$. But for $\ell+1 \leqslant j \leqslant \ell+m-1$, the condition $x^{2} \equiv 1\left(\pi^{j-\ell}\right)$ implies $x-\bar{x} \equiv 0(\pi)$, thus all terms $\chi(\nu(x-\bar{x}))$ with $\chi \neq 1$ vanish. We isolate the term corresponding to $j=\ell$ and obtain

$$
\begin{aligned}
\left\langle\theta_{\xi}, \theta_{\xi}\right\rangle= & \frac{\alpha}{3 \zeta_{k}(2)} \frac{\mathcal{N}(\pi)}{\mathcal{N}(\pi)+1} \mathcal{N}(\pi)^{M-m}\left(\sum_{j \geqslant \ell+m} \sum_{\substack{x\left(\pi^{m}\right) \\
x^{2} \equiv 1\left(\pi^{m}\right)}}^{\times} \mathcal{N}(\pi)^{-j / 3}\left(\frac{x}{\pi^{j}}\right)_{3}\right. \\
& +\varphi\left(\pi^{m}\right)^{-1} \mathcal{N}(\pi)^{-\ell / 3} \sum_{x\left(\pi^{m}\right)}^{\times}\left\{1+\left(\frac{\bar{\nu}(\overline{x-\bar{x}}}{\pi}\right)_{3}+\left(\frac{\nu(x-\bar{x}}{\pi}\right)_{3}\right\}\left(\frac{x}{\pi^{\ell}}\right)_{3} \\
& \left.+\sum_{j=\ell+1}^{\ell-1+m} \varphi\left(\pi^{m-(j-l)}\right)^{-1} \mathcal{N}(\pi)^{-j / 3} \sum_{\substack{x\left(\pi^{m}\right) \\
x^{2} \equiv 1\left(\pi^{j-\ell}\right)}}^{\times}\left(\frac{x}{\pi^{j}}\right)_{3}\right) .
\end{aligned}
$$

In the first term, only $x=1$ and $x=-1$ contribute; in the second term, the dependence is in $x$ $(\bmod \pi)$. Taking into account all these considerations, we now conclude that

$$
\begin{aligned}
& \left\langle\theta_{\xi}, \theta_{\xi}\right\rangle=\frac{\alpha}{3 \zeta_{k}(2)} \frac{\mathcal{N}(\pi)}{\mathcal{N}(\pi)+1} \mathcal{N}(\pi)^{M-m}\left(2 \sum_{j \geqslant \ell+m} \mathcal{N}(\pi)^{-j / 3}\right. \\
& +\frac{\mathcal{N}(\pi)^{1-m}}{\mathcal{N}(\pi)-1} \mathcal{N}(\pi)^{-\ell / 3} \mathcal{N}(\pi)^{m-1} \sum_{x(\pi)}^{\times}\left\{\left(\frac{x^{\ell}}{\pi}\right)_{3}+\overline{\left(\frac{\nu\left(x^{2 \ell+1}-x^{2 \ell-1}\right.}{\pi}\right)_{3}}+\left(\frac{\nu\left(x^{\ell+1}-x^{\ell-1}\right.}{\pi}\right)_{3}\right\} \\
& \left.+\sum_{j=\ell+1}^{\ell-1+m} \frac{\mathcal{N}(\pi)^{-m-\ell+j+1}}{\mathcal{N}(\pi)-1} \mathcal{N}(\pi)^{-j / 3} 2 \mathcal{N}(\pi)^{m-(j-\ell)}\right) .
\end{aligned}
$$

For $m \geqslant 2$, the third sum is not empty, and takes the value

$$
2 \frac{\mathcal{N}(\pi)^{1-\ell / 3}\left(1-\mathcal{N}(\pi)^{(1-m) / 3}\right)}{(\mathcal{N}(\pi)-1)\left(\mathcal{N}(\pi)^{1 / 3}-1\right)} .
$$

Since this expression is 0 for $m=1$, we do not need to specify whether $m \geqslant 2$ or not. This proves Proposition 3.3 if $1 \leqslant \ell \leqslant h-1$, and it remains to treat the case $\ell=h$, in which the equality to be shown is

$$
\operatorname{Res}_{s=4 / 3}\left(\sum_{\substack{c \equiv 0(3) \\ c \equiv 0\left(\pi^{h}\right)}}^{\prime} \mathcal{N}(c)^{-s} \sum_{\substack{d(3 c) \\ d \equiv 1(3)}}\left(\frac{c}{d}\right)_{3}\right)=\operatorname{Res}_{s=4 / 3}\left(\sum_{\substack{c \equiv 1\left(3 \\ c \equiv 0\left(\pi^{h}\right)\right.}}^{\prime} \mathcal{N}(c)^{-s} \sum_{\substack{d(3 c) \\ d \equiv-1(3)}}\left(\frac{c}{d}\right)_{3}\right) .
$$

From Lemma 3.8 , this equality is equivalent to

$$
\begin{gathered}
\operatorname{Res}_{s=4 / 3}\left(\sum_{u= \pm 1} \sum_{i \geq 1} \sum_{3 j \geqslant h} \sum_{\substack{c \equiv 1(3) \\
(c, \pi)=1}} \mathcal{N}\left(u \lambda^{3 i} \pi^{3 j} c^{3}\right)^{-s} \varphi\left(c^{3}\right) \varphi\left(\pi^{3 j}\right) \mathcal{N}(\lambda)^{3 i}\right) \\
=\operatorname{Res}_{s=4 / 3}\left(\sum_{\substack { 3 j \geqslant h \\
\begin{subarray}{c}{c \equiv 1(3) \\
(c, \pi)=1{ 3 j \geqslant h \\
\begin{subarray} { c } { c \equiv 1 ( 3 ) \\
( c , \pi ) = 1 } }\end{subarray}} \mathcal{N}\left(\pi^{3 j} c^{3}\right)^{-s} \varphi\left(c^{3}\right) \varphi\left(\pi^{3 j}\right)\right) .
\end{gathered}
$$


This is clear from Lemma3.9, and thus we obtain

$$
\begin{aligned}
\left\langle\theta_{\xi}, \theta_{\xi}\right\rangle & =\frac{\alpha}{3 \zeta_{k}(2)} \frac{\mathcal{N}(\pi)}{\mathcal{N}(\pi)+1} \sum_{3 j \geqslant h} \mathcal{N}(\pi)^{-4 j} \varphi\left(\pi^{3 j}\right) \\
& =\frac{\alpha}{3 \zeta_{k}(2)} \frac{\mathcal{N}(\pi)-1}{\mathcal{N}(\pi)+1} \sum_{3 j \geqslant h} \mathcal{N}(\pi)^{-j}=\frac{\alpha}{3 \zeta_{k}(2)} \frac{\mathcal{N}(\pi)^{1-\lceil h / 3\rceil}}{\mathcal{N}(\pi)+1} .
\end{aligned}
$$

Proposition 3.4. Let $\xi=(\ell, \nu)$. Then if $\ell=0$ or if $\ell=h$, the four theta functions associated to $\xi$ are equal.

Proof. We already saw that $\theta_{\xi}=\theta_{\xi}^{\prime}$ in Proposition 3.3. It remains to show that for $\ell=0$ or $\ell=h, \theta_{\alpha_{1} \xi}=\theta_{\alpha_{2} \xi}$, for any $\alpha_{1}, \alpha_{2} \in\{ \pm 1,3\}$. For that purpose, we shall show that the expression $\left\langle\theta_{\alpha_{1} \xi}, \theta_{\alpha_{2} \xi}\right\rangle$ is independent of $\alpha_{1}$ and $\alpha_{2}$. Let us start with $\ell=0$. Then, using the same decomposition of the Gauß sum as in (3.2.14) we obtain by Lemma 3.7 that

$$
\begin{aligned}
\left\langle\theta_{\alpha \xi}, \theta_{\beta \xi}\right\rangle= & \operatorname{Res}_{s=4 / 3}\left(\sum_{c \equiv \alpha_{2}-\alpha_{1}(3)}^{\prime} \mathcal{N}(c)^{-s} \sum_{\begin{array}{c}
d\left(3 \pi h_{c}\right) \\
d \equiv 1(3) \\
d \neq \bar{\alpha}_{2} c(\pi)
\end{array}}\left(\frac{c}{d}\right)_{3}\right) \\
= & \operatorname{Res}_{s=4 / 3}\left(\sum_{u \in\{ \pm 1\}} \sum_{\substack{i \geqslant 0 \\
u \lambda^{3 i} \equiv \alpha_{2}-\alpha_{1}(3)}} \sum_{c \equiv 1(3)} \mathcal{N}\left(u \lambda^{3 i} c^{3}\right)^{-s} \varphi\left(c^{3}\right) \mathcal{N}(\lambda)^{3 i}\right. \\
& \left.\left\{\sum_{j \geqslant 0} \varphi\left(\pi^{3 j+h}\right) \mathcal{N}(\pi)^{-3 j s}-\sum_{j \geqslant 0}\left(\frac{u \lambda^{i} c^{3} \pi^{j}}{\pi^{j}}\right)_{3} \mathcal{N}(\pi)^{-j s} \mathcal{N}(\pi)^{h+j-1}\right\}\right) .
\end{aligned}
$$

Lemma 3.9 shows the independence in $\alpha_{1}$ and $\alpha_{2}$. Similarly, in the case $\ell=h$, we have

$$
\begin{aligned}
& \left\langle\theta_{\alpha_{1} \pi^{h}}, \theta_{\alpha_{2} \pi^{h}}\right\rangle=\operatorname{Res}_{s=4 / 3}\left(\sum_{\substack{c \equiv \alpha_{2}-\alpha_{1}(3) \\
c \equiv 0\left(\pi^{h}\right)}}^{\prime} \mathcal{N}(c)^{-s} \sum_{\substack{d(3 c) \\
d \equiv 1(3)}}\left(\frac{c}{d}\right)_{3}\right) \\
& =\operatorname{Res}_{s=4 / 3}\left(\sum_{u \in\{ \pm 1\}} \sum_{\substack{i \geqslant 0 \\
u \lambda^{3 i} \equiv \alpha_{2}-\alpha_{1}(3)}} \sum_{3 j \geqslant h} \sum_{c \equiv 1(3)} \mathcal{N}\left(u \lambda^{3 i} c^{3} \pi^{3 j}\right)^{-s} \varphi\left(c^{3}\right) \varphi\left(\pi^{3 j}\right) \mathcal{N}(\lambda)^{3 i}\right) .
\end{aligned}
$$

Again, Lemma 3.9 allows us to finish the proof of Proposition 3.4 .

Proposition 3.5. Let $1 \leqslant \ell \leqslant h-1$, and let $m(\ell)$ and $M(\ell)$ be defined as in 3.2.7) $p$. 100. Let $\alpha_{1}, \alpha_{2} \in\{ \pm 1,3\}$ and let $\nu_{1}, \nu_{2} \in \mathcal{P}_{\ell}$. Then 


$$
\begin{aligned}
& \frac{3 \zeta_{k}(2)}{2 \alpha} \mathcal{N}(\pi)^{m-M-1+\ell / 3}\left(\mathcal{N}(\pi)^{2}-1\right)\left\langle\theta_{\alpha_{1} \pi^{\ell} \nu_{1}}, \theta_{\alpha_{1} \pi^{\ell} \nu_{2}}\right\rangle= \\
& \left\{\begin{array}{lll}
\left(\frac{\alpha_{1} \nu_{1}}{\pi}\right)_{3} s\left(\left(\alpha_{1} \nu_{1}\right)^{-1} \alpha_{2} \nu_{2}\right)+\left(\frac{\alpha_{2} \nu_{2}}{\pi}\right)_{3}^{2} \bar{s}\left(\alpha_{1} \nu_{1}\left(\alpha_{2} \nu_{2}\right)^{-1}\right) & \text { if } \ell \equiv 1 & (\bmod 3) \\
\left(\frac{\alpha_{1} \nu_{1}}{\pi}\right)_{3}^{2} \bar{s}\left(\left(\alpha_{1} \nu_{1}\right)^{-1} \alpha_{2} \nu_{2}\right)+\left(\frac{\alpha_{2} \nu_{2}}{\pi}\right)_{3} s\left(\alpha_{1} \nu_{1}\left(\alpha_{2} \nu_{2}\right)^{-1}\right) & \text { if } \ell \equiv-1 & (\bmod 3) \\
\mathcal{N}(\pi)-1+2 \Re\left(\left(\frac{\nu_{1} \alpha_{1}}{\pi}\right)_{3} t\left(\nu_{1} \alpha_{1}\left(\nu_{2} \alpha_{2}\right)^{-1}\right)\right) & \text { if } \ell \equiv 0 & (\bmod 3)
\end{array}\right. \\
& +\delta\left(\frac{\alpha_{2} \nu_{2}\left(\alpha_{1} \nu_{1}\right)^{-1}}{\pi}\right)_{3}^{\ell}\left(\left(\frac{\alpha_{2} \nu_{2}\left(\alpha_{1} \nu_{1}\right)^{-1}}{\pi}\right)_{3}^{2} \mathcal{N}(\pi)^{1 / 3}-1\right)^{-1}\left(\mathcal{N}(\pi)-\mathcal{N}(\pi)^{(1-m) / 3}\left(\frac{\alpha_{2} \nu_{2}\left(\alpha_{1} \nu_{1}\right)^{-1}}{\pi}\right)_{3}^{m-1}\right) .
\end{aligned}
$$

Here, $\delta$ is equal to 1 if $\left(\frac{\alpha_{1} \nu_{1}}{\pi}\right)_{2}=\left(\frac{\alpha_{2} \nu_{2}}{\pi}\right)_{2}$ and $\delta=0$ otherwise.

Corollary 3.1. Let $\ell$ be fixed and let $\nu_{j}, j=1, \ldots, 6$, be some elements of $\mathcal{P}_{\ell}$ such that $\left(\frac{\nu_{i}}{\pi}\right)_{3}\left(\frac{\nu_{i}}{\pi}\right)_{2} \neq$ $\left(\frac{\nu_{j}}{\pi}\right)_{3}\left(\frac{\nu_{j}}{\pi}\right)_{2}$ if $i \neq j$ or equivalently, $\left\{\nu_{j}\right\}_{j=1, \ldots, 6}$ is a set of representative of

$$
\{x \in R,(x, \pi)=1\} / \operatorname{ker}(\phi),
$$

where $\phi(x)=\left(\frac{x}{\pi}\right)_{3}\left(\frac{x}{\pi}\right)_{2}$. Then $\theta_{\pi^{\ell} \nu_{1}}, \ldots, \theta_{\pi^{\ell} \nu_{6}}$ is a generator system of the space generated by $\left\{\theta_{\pi^{\ell}}, \theta_{-\pi^{\ell} \nu}, \theta_{3 \pi^{\ell} \nu}: \nu \in \mathcal{P}_{\ell}\right\}$.

Proof. (of Proposition 3.5)

Let $1 \leqslant \ell \leqslant h-1$ be fixed. Let $\xi_{1}=\left(\ell, \nu_{1}\right.$ and $\xi_{2}=\left(\ell, \nu_{2}\right)$; recall that the lattice associated to both cusps $\left(\alpha_{1} \pi^{\ell} \nu_{1}\right)^{-1}$ and $\left(\alpha_{2} \pi^{\ell} \nu_{2}\right)^{-1}$ is $\Lambda=\Lambda_{\ell}=3 \pi^{M} R$. By Lemma 3.7

$$
\begin{aligned}
& \left\langle\theta_{\alpha_{1} \xi_{1}}, \theta_{\alpha_{1} \xi_{2}}\right\rangle=\operatorname{Res}_{s=4 / 3}\left(\sum_{\substack{c \equiv \alpha_{2}-\alpha_{1}(3) \\
c \equiv 0\left(\pi^{\ell}\right)}}^{\prime} \mathcal{N}(c)^{-s} \sum_{\substack{d(\Lambda c) \\
d \equiv \equiv(3) \\
d \alpha_{2} \nu_{2}-\bar{d} \alpha_{1} \nu_{1} \equiv \frac{c}{\pi^{\ell}}\left(\pi^{m}\right)}}\left(\frac{c}{d}\right)_{3}\right)
\end{aligned}
$$

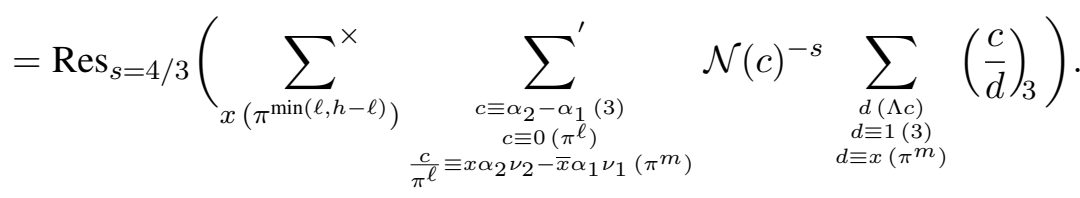

Since we assume $1 \leqslant \ell \leqslant h-1$, it holds $m=\min (\ell, h-\ell) \geqslant 1$; in particular, we can use Lemma 3.8, and evaluate the inner sum by

$$
\begin{aligned}
& \left\langle\theta_{\alpha_{1} \xi_{1}}, \theta_{\alpha_{2} \xi_{2}}\right\rangle=
\end{aligned}
$$

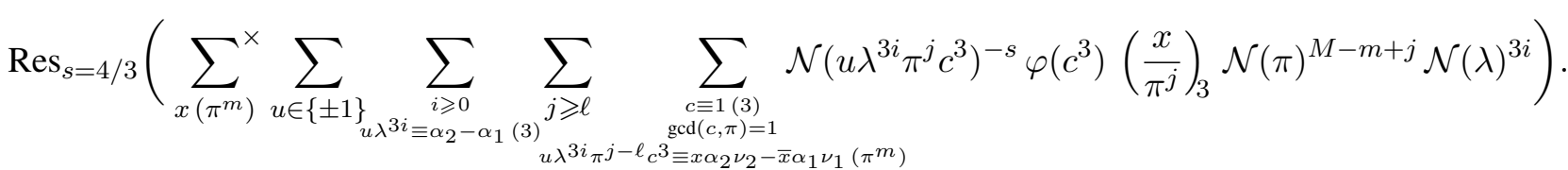

For $j$ big enough, i.e. $j \geqslant \ell+m$, the condition $u \lambda^{3 i} \pi^{j-\ell} c^{3} \equiv x \alpha_{2} \nu_{2}-\bar{x} \alpha_{1} \nu_{1}\left(\pi^{m}\right)$ becomes $x^{2} \equiv \alpha_{1} \nu_{1} \overline{\alpha_{2} \nu_{2}}\left(\bmod \pi^{m}\right)$, but for $\ell \leqslant j \leqslant \ell+m-1$, it becomes $x^{2} \equiv \alpha_{1} \nu_{1} \overline{\alpha_{2} \nu_{2}}\left(\bmod \pi^{j-\ell}\right)$ 
and $u \lambda^{3 i} c^{3} \equiv \pi^{-(j-\ell)}\left(x \alpha_{2} \nu_{2}-\bar{x} \alpha_{1} \nu_{1}\right)\left(\bmod \pi^{m-(j-\ell)}\right)$. According to this, we split the sum over the $j$ 's and obtain

$$
\begin{aligned}
& \mathcal{N}(\pi)^{m-M}\left\langle\theta_{\alpha_{1} \pi^{\ell} \nu_{1}}, \theta_{\alpha_{1} \pi^{\ell} \nu_{2}}\right\rangle=\operatorname{Res}_{s=4 / 3}( \\
& \sum_{\ell \leqslant j \leqslant \ell+m-1} \sum_{\substack{x\left(\pi^{m}\right) \\
x^{2} \equiv \alpha_{1} \nu_{1} \alpha_{2} \nu_{2}}}^{\times} \sum_{\substack{u= \pm 1 \\
\left(\pi^{j}-\ell\right)}} \sum_{\substack{i \geqslant 0 \\
u \lambda^{3 i} \equiv \alpha_{2}-\alpha_{1}(3) \\
u \lambda^{3 i} c^{3} \equiv \pi^{-(j-\ell)}}} \sum_{\substack{c \equiv \pm(3) \\
(c, \pi)=1 \\
\left(x \alpha_{2} \nu_{2}-\bar{x} \alpha_{1} \nu_{1}\right)\left(\pi^{m-(j-\ell)}\right)}} \mathcal{N}\left(u \lambda^{3 i} \pi^{j} c^{3}\right)^{-s} \varphi\left(c^{3}\right)\left(\frac{x}{\pi^{j}}\right)_{3} \mathcal{N}(\pi)^{j} \mathcal{N}(\lambda)^{3 i} \\
& \left.+\sum_{j \geqslant m+\ell} \sum_{\substack{x\left(\pi^{m}\right) \\
x^{2} \equiv \alpha_{1} \bar{\alpha}_{1} \bar{\alpha}_{2} \nu_{2}\left(\pi^{m}\right)}}^{\times} \sum_{u= \pm 1} \sum_{\substack{i \geqslant 0 \\
u \lambda^{3 i} \equiv \alpha_{2}-\alpha_{1}(3)}} \sum_{\substack{c \equiv 1(3) \\
(c, \pi)=1}} \mathcal{N}\left(u \lambda^{3 i} \pi^{j} c^{3}\right)^{-s} \varphi\left(c^{3}\right)\left(\frac{x}{\pi^{j}}\right)_{3} \mathcal{N}(\pi)^{j} \mathcal{N}(\lambda)^{3 i}\right) .
\end{aligned}
$$

We replace the congruence condition over the $c$ 's by the introduction of a characters sum; more precisely,

$$
\begin{gathered}
\sum_{\substack{c \equiv 1(3) \\
(c, \pi)=1 \\
\left(x \alpha_{2} \nu_{2}-\bar{x} \alpha_{1} \nu_{1}\right)\left(\pi^{m-(j-\ell)}\right)}} \mathcal{N}\left(u \lambda^{3 i} \pi^{j} c^{3}\right)^{-s} \varphi\left(c^{3}\right) \\
=\varphi\left(\pi^{m-(j-\ell)}\right)^{-1} \sum_{\chi\left(\pi^{m-(j-\ell)}\right)} \sum_{\substack{c \equiv 1(3) \\
(c, \pi)=1}} \chi\left(\overline{u \lambda^{3 i} c^{3} \equiv c^{3}} \pi^{-(j-\ell)}\left(x \alpha_{2} \nu_{2}-\bar{x} \alpha_{1} \nu_{1}\right)\right) \mathcal{N}\left(u \lambda^{3 i} \pi^{j} c^{3}\right)^{-s} \varphi\left(c^{3}\right) .
\end{gathered}
$$

By Lemma 3.9, only cubic characters contribute non trivially, but the only cubic characters modulo $\pi^{m-(j-\ell)}$ are the powers of the cubic Legendre symbol. Therefore, we apply Lemma 3.8 with $g=\pi$ and with, either $f=1$ or $f=\pi$. Thus

$$
\begin{gathered}
\mathcal{N}(\pi)^{m-M}\left\langle\theta_{\alpha_{1} \pi^{\ell} \nu_{1}}, \theta_{\alpha_{1} \pi^{\ell} \nu_{2}}\right\rangle= \\
\frac{\alpha}{(3 \zeta(2)} \frac{\mathcal{N}(\pi)}{\mathcal{N}(\pi)+1}\left(\sum_{\ell \leqslant j \leqslant m-1+\ell} \varphi\left(\pi^{m-(j-l)}\right)^{-1} \sum_{\substack{\chi\left(\pi^{m-(j-\ell)}\right) \\
\chi^{3}=1}} \sum_{x^{2} \equiv \alpha_{1} \nu_{1} \bar{\alpha}_{2^{m} \nu_{2}}\left(\pi^{j}-\ell\right)} \sum_{u= \pm 1} \sum_{\substack{i \geqslant 0 \\
u \lambda^{3 i} \equiv \alpha_{2}-\alpha_{1}(3)}}\right. \\
\chi\left(\overline{u \lambda^{3 i}} \pi^{-(j-\ell)}\left(x \alpha_{2} \nu_{2}-\bar{x} \alpha_{1} \nu_{1}\right)\right) \mathcal{N}\left(u \lambda^{3 i} \pi^{j}\right)^{-1 / 3}\left(\frac{x}{\pi^{j}}\right)_{3} \\
\left.+\sum_{j \geqslant m+\ell} \sum_{\substack{x\left(\pi^{m}\right) \\
x^{2} \equiv \alpha_{1} \nu_{1} \bar{\alpha}_{2} \nu_{2}\left(\pi^{m}\right)}}^{\times} \sum_{u= \pm 1} \sum_{\substack{i \geqslant 0 \\
u \lambda^{3 i} \equiv \alpha_{2}-\alpha_{1}(3)}} \mathcal{N}\left(u \lambda^{3 i} \pi^{j}\right)^{-1 / 3}\left(\frac{x}{\pi^{j}}\right)_{3}\right)
\end{gathered}
$$

Since we deal only with cubic characters we have $\chi\left(u \lambda^{3 i}\right)=1$, and by Lemma3.9, we are left with 


$$
\begin{gathered}
\mathcal{N}(\pi)^{m-M}\left\langle\theta_{\alpha_{1} \xi_{1}}, \theta_{\alpha_{2} \xi_{2}}\right\rangle=\frac{\alpha}{3 \zeta_{k}(2)} \frac{\mathcal{N}(\pi)}{\mathcal{N}(\pi)+1}\left(\sum_{j \geqslant m+\ell} \sum_{\substack{\left.x \pi^{m}\right) \\
x^{2} \equiv \alpha_{1} \nu_{1} \bar{\alpha}_{2} \nu_{2} \\
\left(\pi^{m}\right)}}^{\times} \mathcal{N}(\pi)^{-j / 3}\left(\frac{x}{\pi^{j}}\right)_{3}\right. \\
\left.+\sum_{\ell \leqslant j \leqslant m-1+\ell} \varphi\left(\pi^{m-(j-l)}\right)^{-1} \sum_{\substack{\chi\left(\pi^{m-(j-\ell)} \\
\chi^{3}=1\right.}} \sum_{\substack{\left.x=\pi^{m}\right) \\
x^{2} \equiv \alpha_{1} \nu_{1} \bar{\alpha}_{2} \nu_{2}\left(\pi^{j-\ell}\right)}}^{\times} \chi\left(\frac{x \alpha_{2} \nu_{2}-\bar{x} \alpha_{1} \nu_{1}}{\pi^{j-\ell}}\right) \mathcal{N}(\pi)^{-j / 3}\left(\frac{x}{\pi^{j}}\right)_{3}\right) .
\end{gathered}
$$

We remark that except for the term corresponding to $j=\ell$, all the other terms vanish if $\alpha_{1} \nu_{1} \overline{\alpha_{2} \nu_{2}}$ is not a square modulo $\pi$. Assuming that $\alpha_{1} \nu_{1} \overline{\alpha_{2} \nu_{2}} \equiv y^{2}\left(\bmod \pi^{m}\right)$ and because $\chi$ is defined modulo $\pi$, we have

$$
\sum_{\substack{x\left(\pi^{m}\right) \\ x^{2} \equiv \alpha_{1} \nu_{1} \bar{\alpha}_{2} \nu_{2} \\\left(\pi^{j-\ell}\right)}}^{\times} \chi\left(\frac{x \alpha_{2} \nu_{2}-\bar{x} \alpha_{1} \nu_{1}}{\pi^{j-\ell}}\right)\left(\frac{x}{\pi^{j}}\right)_{3}=\sum_{\substack{x\left(\pi^{m}\right) \\ x^{2} \equiv \alpha_{1} \nu_{1} \bar{\alpha}_{2} \nu_{2}\left(\pi^{j-\ell}\right)}}^{\times} \bar{\chi}(x) \chi\left(\frac{x^{2} \alpha_{2} \nu_{2}-\alpha_{1} \nu_{1}}{\pi^{j-\ell}}\right)\left(\frac{x}{\pi^{j}}\right)_{3}
$$

Writing $x= \pm y+x^{\prime} \pi^{j-\ell}$ with $x^{\prime}$ modulo $\pi^{m-(j-\ell)}$ and because $\chi$ is defined modulo $\pi$, we have $\bar{\chi}(x)=\bar{\chi}(y),\left(\frac{x}{\pi^{j}}\right)_{3}=\left(\frac{y}{\pi^{j}}\right)_{3}$ and $x^{2} \alpha_{2} \nu_{2}-\alpha_{1} \nu_{1} \equiv 2 y x^{\prime} \pi^{j-\ell}\left(\bmod \pi^{j-\ell+1}\right)$. Taking the sum over $x^{\prime}$ modulo $\pi^{m-(j-\ell)}$ shows that $\chi$ has to be trivial whenever $\ell+1 \leqslant j \leqslant \ell+m-1$. The resulting term in 3.2.16 for one of those $j$ 's is then

$$
\mathcal{N}(\pi)^{-j / 3} \varphi\left(\pi^{m-(j-l)}\right)^{-1} \sum_{\substack{x\left(\pi^{m}\right) \\ x^{2} \equiv \alpha_{1} \nu_{1} \bar{\alpha}_{2} \nu_{2} \\\left(\pi^{j-\ell}\right)}}^{\times}\left(\frac{x}{\pi^{j}}\right)_{3}=2\left(\frac{\alpha_{2} \nu_{2} \overline{\alpha_{1} \nu_{1}}}{\pi}\right)^{j} \frac{\mathcal{N}(\pi)^{1-j / 3}}{\mathcal{N}(\pi)-1}
$$

and, under the condition $m \geqslant 2$, the sum over $\ell+1 \leqslant j \leqslant \ell+m-1$ in (3.2.16) makes sense, and, if $\alpha_{1} \nu_{1} \overline{\alpha_{2} \nu_{2}} \equiv y^{2}\left(\bmod \pi^{m}\right)$, it is given by

$2\left(\frac{\alpha_{2} \nu_{2} \overline{\alpha_{1} \nu_{1}}}{\pi}\right)^{\ell} \frac{\mathcal{N}(\pi)^{1-\ell / 3}}{\mathcal{N}(\pi)-1}\left(1-\left(\frac{\alpha_{2} \nu_{2} \overline{\alpha_{1} \nu_{1}}}{\pi}\right)_{3}^{m-1} \mathcal{N}(\pi)^{(1-m) / 3}\right)\left(\left(\frac{\alpha_{1} \nu_{1} \overline{\alpha_{2} \nu_{2}}}{\pi}\right)_{3} \mathcal{N}(\pi)^{1 / 3}-1\right)^{-1}$

Since this expression vanishes for $m=1$, we do not need to specify whether $m \geqslant 2$ or not. (Recall that our assumption on $\ell$ implies $m \geqslant 1$ always.) In (3.2.16), it remains to treat the sum over the $j$ 's bigger than $\ell+m$ and to treat the single term corresponding to $j=\ell$. The former is equal to

$$
2\left(\frac{\alpha_{2} \nu_{2} \overline{\alpha_{1} \nu_{1}}}{\pi}\right)_{3}^{\ell+m-1} \mathcal{N}(\pi)^{(1-\ell-m) / 3}\left(\left(\frac{\alpha_{1} \nu_{1} \overline{\alpha_{2} \nu_{2}}}{\pi}\right)_{3} \mathcal{N}(\pi)^{1 / 3}-1\right)^{-1}
$$

and the latter is given by 


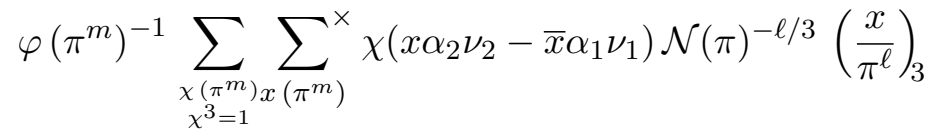

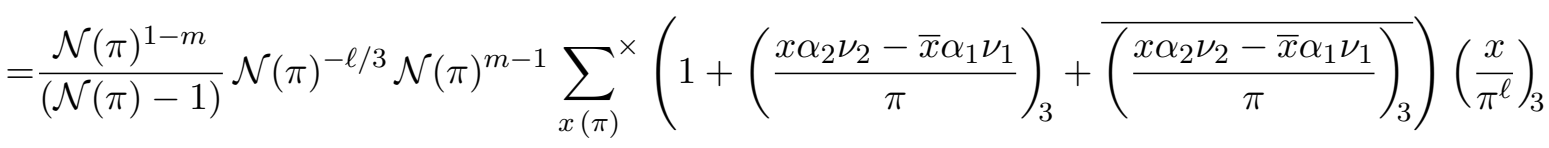

$$
\begin{aligned}
& =\frac{\mathcal{N}(\pi)^{-\ell / 3}}{(\mathcal{N}(\pi)-1)}\left(\sum_{x(\pi)}^{\times}\left(\frac{x}{\pi^{\ell}}\right)_{3}+\sum_{x(\pi)}^{\times}\left(\frac{x^{\ell+1} \alpha_{2} \nu_{2}-x^{\ell-1} \alpha_{1} \nu_{1}}{\pi}\right)_{3}+\sum_{x(\pi)}^{\times} \overline{\left(\frac{x^{2 \ell+1} \alpha_{2} \nu_{2}-x^{2 \ell-1} \alpha_{1} \nu_{1}}{\pi}\right)_{3}}\right) \\
& =\frac{\mathcal{N}(\pi)^{-\ell / 3}}{(\mathcal{N}(\pi)-1)}\left\{\begin{array}{lll}
\left(\frac{\alpha_{1} \nu_{1}}{\pi}\right)_{3} s\left(\overline{\alpha_{1} \nu_{1}} \alpha_{2} \nu_{2}\right)+\left(\frac{\overline{\alpha_{2} \nu_{2}}}{\pi}\right)_{3} \overline{s\left(\alpha_{1} \nu_{1} \overline{\alpha_{2} \nu_{2}}\right)} & \text { if } \ell \equiv 1 \quad(\bmod 3) \\
\overline{\left(\frac{\alpha_{1} \nu_{1}}{\pi}\right)_{3} s\left(\overline{\alpha_{1} \nu_{1}} \alpha_{2} \nu_{2}\right)}+\left(\frac{\alpha_{2} \nu_{2}}{\pi}\right)_{3} s\left(\alpha_{1} \nu_{1} \overline{\alpha_{2} \nu_{2}}\right) & \text { if } \ell \equiv-1 \quad(\bmod 3) \\
\mathcal{N}(\pi)-1+2 \Re\left(\left(\frac{\nu_{1} \alpha_{1}}{\pi}\right)_{3} t\left(\nu_{1} \alpha_{1} \overline{\nu_{2} \alpha_{2}}\right)\right) & \text { if } \ell \equiv 0 \quad(\bmod 3)
\end{array}\right.
\end{aligned}
$$

We conclude the proof of the Proposition 3.5 by putting together (3.2.17), 3.2.18) and 3.2 .19 ) in the equation 3.2.16.

Proposition 3.6. Let $\ell_{1}<\ell_{2}$ and let $\nu_{1} \in \mathcal{P}_{\ell_{1}}$ and $\nu_{2} \in \mathcal{P}_{\ell_{2}}$. Then

$$
\left\langle\theta_{\pi^{\ell_{1}}}, \theta_{\pi^{\ell_{2} \nu_{2}}}\right\rangle=\frac{\alpha}{3 \zeta(2)} \frac{\mathcal{N}(\pi)^{M\left(\ell_{2}\right)+1-m\left(\ell_{1}\right)-\ell_{1} / 3}}{\mathcal{N}(\pi)+1}\left(\frac{\nu_{1}}{\pi}\right)_{3}^{\ell_{1}} .
$$

Proof. Assume first that $\ell_{1}>0$. Let $m=\min \left(\ell_{1}, h-\ell_{1}\right)$ and $M=\operatorname{Max}\left(0, h-2 \ell_{2}\right)$. By Lemma 3.6, we have

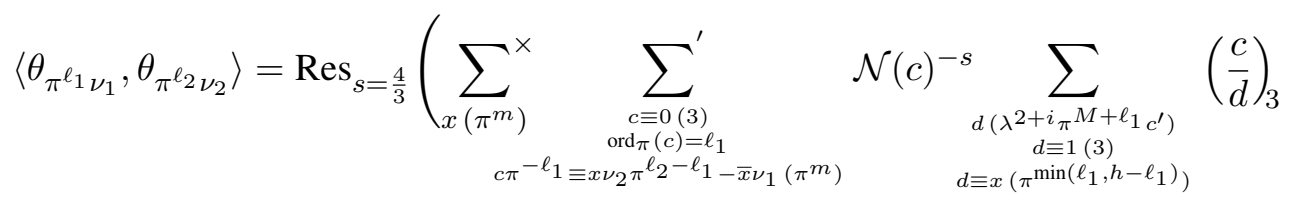

We use Lemma 3.7 and, introducing a characters sum, this is equal to

$$
\begin{aligned}
& \mathcal{N}(\pi)^{M-m-\ell_{1} / 3} \operatorname{Res}_{s=4 / 3}\left(\sum_{x\left(\pi^{m}\right)}^{\times}\left(\frac{x}{\pi}\right)_{3}^{\ell_{1}} \sum_{u= \pm 1} \sum_{u \lambda^{3 i} \equiv 0(3)} \sum_{\substack{c \equiv 1(3) \\
(c, \pi)=1 \\
u \lambda^{3 i} c^{3} \equiv x \nu_{2} \pi^{\ell}-\ell_{1}-\bar{x} \nu_{1}\left(\pi^{m}\right)}} \mathcal{N}\left(u \lambda^{3 i} c^{3}\right)^{-s} \varphi\left(c^{3}\right) \mathcal{N}(\lambda)^{3 i}\right) \\
& =\mathcal{N}(\pi)^{M-m-\ell_{1} / 3} \varphi\left(\pi^{m}\right)^{-1} \sum_{\chi\left(\pi^{m}\right)} \operatorname{Res}_{s=4 / 3}\left(\sum_{x\left(\pi^{m}\right)}^{\times}\left(\frac{x}{\pi}\right)_{3}^{\ell_{1}} \sum_{u= \pm 1} \sum_{u \lambda^{3 i} \equiv 0} \sum_{\substack { c=1(3) \\
\begin{subarray}{c}{c=1(3)=1 \\
(c, \pi)=1{ c = 1 ( 3 ) \\
\begin{subarray} { c } { c = 1 ( 3 ) = 1 \\
( c , \pi ) = 1 } }\end{subarray}} \mathcal{N}\left(u \lambda^{3 i} c^{3}\right)^{-s} \varphi\left(c^{3}\right) \mathcal{N}(\lambda)^{3 i}\right) .
\end{aligned}
$$

By Lemma 3.8 and Lemma 3.9, we obtain 


$$
\begin{aligned}
& \frac{1}{3 \zeta_{k}(2)} \frac{\mathcal{N}(\pi)^{M-2 m-\ell_{1} / 3+2}}{\mathcal{N}(\pi)^{2}-1} \sum_{\substack{\chi\left(\pi^{m}\right) \\
\chi^{3}=1}} \sum_{x\left(\pi^{m}\right)}^{\times}\left(\frac{x}{\pi}\right)_{3}^{\ell_{1}} \bar{\chi}\left(x \nu_{2} \pi^{\ell_{2}-\ell_{1}}-\bar{x} \nu_{1}\right) \\
& =\frac{1}{3 \zeta_{k}(2)} \frac{\mathcal{N}(\pi)^{M-2 m-\ell_{1} / 3+2}}{\mathcal{N}(\pi)^{2}-1} \mathcal{N}(\pi)^{m-1} \sum_{\substack{\chi\left(\pi^{m}\right) \\
\chi^{3}=1}} \sum_{x(\pi)}^{\times}\left(\frac{x}{\pi}\right)_{3}^{\ell_{1}} \chi\left(x \overline{\nu_{1}}\right) \\
& =\frac{1}{3 \zeta_{k}(2)} \frac{\mathcal{N}(\pi)^{M-m-\ell_{1} / 3+1}}{\mathcal{N}(\pi)^{2}-1}\left(\sum_{x(\pi)}^{\times}\left(\frac{x}{\pi}\right)_{3}^{\ell_{1}}+\sum_{x(\pi)}^{\times}\left(\frac{x}{\pi}\right)_{3}^{\ell_{1}+1}\left(\frac{\overline{\nu_{1}}}{\pi}\right)_{3}+\sum_{x(\pi)}^{\times}\left(\frac{x}{\pi}\right)_{3}^{\ell_{1}-1}\left(\frac{\nu_{1}}{\pi}\right)_{3}\right) \\
& =\frac{1}{3 \zeta_{k}(2)} \frac{\mathcal{N}(\pi)^{M-m-\ell_{1} / 3+1}}{\mathcal{N}(\pi)+1}\left(\frac{\nu_{1}}{\pi}\right)_{3}^{\ell_{1}}
\end{aligned}
$$

If $\ell_{1}=0$, then

$$
\begin{aligned}
& \left\langle\theta_{\pi^{\ell_{1} \nu_{1}}}, \theta_{\pi^{\ell_{2} \nu_{2}}}\right\rangle=\operatorname{Res}_{s=4 / 3}\left(\sum_{\substack{c \equiv 0(3) \\
\text { ord } \pi(c)=0}}^{\prime} \mathcal{N}(c)^{-s} \sum_{\substack{d\left(\lambda^{2+i} \pi^{M} c_{\left.c^{\prime}\right)} \\
d \equiv 1(3)\right.}}\left(\frac{c}{d}\right)_{3}\right) \\
& =\operatorname{Res}_{s=4 / 3}\left(\sum_{\substack{c \equiv=(3) \\
\text { ord }(c)=0 \\
\text { c cube }}}^{\prime} \mathcal{N}(c)^{-s} \varphi\left(c^{\prime}\right) \mathcal{N}(\pi)^{M} \mathcal{N}(\lambda)^{i}\right) \\
& =\mathcal{N}(\pi)^{M} \operatorname{Res}_{s=4 / 3}\left(\sum_{u= \pm 1} \sum_{u \lambda^{3 i} \equiv 0(3)} \sum_{\substack{c \equiv 1(3) \\
(c, \pi)=1}} \mathcal{N}\left(u \lambda^{3 i} c^{3}\right)^{-s} \varphi\left(c^{3}\right) \mathcal{N}(\lambda)^{3 i}\right) \\
& =\frac{1}{3 \zeta(2)} \frac{\mathcal{N}(\pi)^{M+1}}{\mathcal{N}(\pi)+1} .
\end{aligned}
$$




\subsection{Some conclusions}

Recall that the space of theta functions has been defined at $p .88$, and we later simplified the notations at p.91. From Proposition 3.3. Proposition 3.4 and Proposition 3.5 of the previous section, one deduces that

$$
\operatorname{dim}\left(\Theta\left(\pi^{h}\right)\right) \leqslant 2+6(h-1) .
$$

In the special case $h=1$, one verifies by Proposition 3.3. Proposition 3.6 and the CauchySchwarz criterion that $\theta_{1}$ and $\theta_{\pi^{h}}$ are linearly dependent. This means that

$$
\operatorname{dim}(\Theta(\pi))=1 .
$$

and a generator is the cubic theta function $\theta_{3}(w)$ defined in 3.1.4 of Section 3.1 .2

We now study the cases $h=2$ and $h=3$.We define $\nu_{j}, j=1, \ldots, 6$, such that $\nu_{j} \equiv 1$ $(\bmod 3)$ and

\begin{tabular}{|c|c|c|}
\hline$x$ & $\left(\frac{x}{\pi}\right)_{3}$ & $\left(\frac{x}{\pi}\right)_{2}$ \\
\hline$\nu_{1}$ & 1 & 1 \\
$\nu_{2}$ & $\omega$ & 1 \\
$\nu_{3}$ & $\omega^{2}$ & 1 \\
$\nu_{4}$ & 1 & -1 \\
$\nu_{5}$ & $\omega$ & -1 \\
$\nu_{6}$ & $\omega^{2}$ & -1 \\
\hline
\end{tabular}

Define the constant $c(\ell)=(3 \zeta(2))^{1 / 2} \mathcal{N}(\pi)^{\frac{1}{2}(m(\ell)-M(\ell)-1+\ell / 3)} \sqrt{\mathcal{N}(\pi)+1}$. We make a linear combination of the theta functions as follows:

$$
\theta^{0}=c(0) \theta_{1}, \quad \text { and } \quad \theta^{h}=c(h) \theta_{\pi^{h}} .
$$

For $1 \leqslant \ell \leqslant h-1$,

$$
\begin{aligned}
& \theta_{1}^{\ell}=\frac{c(\ell)}{6}\left(\theta_{\pi^{\ell} \nu_{1}}+\theta_{\pi^{\ell} \nu_{2}}+\theta_{\pi^{\ell} \nu_{3}}+\theta_{\pi^{\ell} \nu_{4}}+\theta_{\pi^{\ell} \nu_{5}}+\theta_{\pi^{\ell} \nu_{6}}\right), \\
& \theta_{2}^{\ell}=\frac{c(\ell)}{6}\left(\theta_{\pi^{\ell} \nu_{1}}+\omega^{2} \theta_{\pi^{\ell} \nu_{2}}+\omega \theta_{\pi^{\ell} \nu_{3}}+\theta_{\pi^{\ell} \nu_{4}}+\omega^{2} \theta_{\pi^{\ell} \nu_{5}}+\omega \theta_{\pi^{\ell} \nu_{6}}\right), \\
& \theta_{3}^{\ell}=\frac{c(\ell)}{6}\left(\theta_{\pi^{\ell} \nu_{1}}+\omega \theta_{\pi^{\ell} \nu_{2}}+\omega^{2} \theta_{\pi^{\ell} \nu_{3}}+\theta_{\pi^{\ell} \nu_{4}}+\omega \theta_{\pi^{\ell} \nu_{5}}+\omega^{2} \theta_{\pi^{\ell} \nu_{6}}\right), \\
& \theta_{4}^{\ell}=\frac{c(\ell)}{6}\left(\theta_{\pi^{\ell} \nu_{1}}+\theta_{\pi^{\ell} \nu_{2}}+\theta_{\pi^{\ell} \nu_{3}}-\theta_{\pi^{\ell} \nu_{4}}-\theta_{\pi^{\ell} \nu_{5}}-\theta_{\pi^{\ell} \nu_{6}}\right), \\
& \theta_{5}^{\ell}=\frac{c(\ell)}{6}\left(\theta_{\pi^{\ell} \nu_{1}}+\omega^{2} \theta_{\pi^{\ell} \nu_{2}}+\omega \theta_{\pi^{\ell} \nu_{3}}-\theta_{\pi^{\ell} \nu_{4}}-\omega^{2} \theta_{\pi^{\ell} \nu_{5}}-\omega \theta_{\pi^{\ell} \nu_{6}}\right), \\
& \theta_{6}^{\ell}=\frac{c(\ell)}{6}\left(\theta_{\pi^{\ell} \nu_{1}}+\omega \theta_{\pi^{\ell} \nu_{2}}+\omega^{2} \theta_{\pi^{\ell} \nu_{3}}-\theta_{\pi^{\ell} \nu_{4}}-\omega \theta_{\pi^{\ell} \nu_{5}}-\omega^{2} \theta_{\pi^{\ell} \nu_{6}}\right) .
\end{aligned}
$$

Write $n=(\mathcal{N}(\pi)-1)^{-1}$ and $J=n J\left(\chi_{3}, \chi_{2}\right)$, according to 3.2 .9$)$. For the case $h=2$, we need the table of scalar products between theta functions for $\ell=1$, and in the case $h=3$, we need the tables corresponding to $\ell=1$ and $\ell=2$; in both cases, one remarks that $m(\ell)=1$. We obtain 


\begin{tabular}{|c|c|c|c|c|c|c|}
\hline$\langle\cdot, \cdot\rangle$ & $\theta_{1}^{1}$ & $\theta_{2}^{1}$ & $\theta_{3}^{1}$ & $\theta_{4}^{1}$ & $\theta_{5}^{1}$ & $\theta_{6}^{1}$ \\
\hline$\theta_{1}^{1}$ & $n \mathcal{N}(\pi)^{1 / 3}$ & $-n$ & 0 & 0 & 0 & 0 \\
\hline$\theta_{2}^{1}$ & $-n$ & $n \mathcal{N}(\pi)^{2 / 3}$ & 0 & 0 & 0 & 0 \\
\hline$\theta_{3}^{1}$ & 0 & 0 & $n$ & 0 & 0 & 0 \\
\hline$\theta_{4}^{1}$ & 0 & 0 & 0 & $n \mathcal{N}(\pi)^{1 / 3}$ & $\overline{J(\pi)}$ & 0 \\
\hline$\theta_{5}^{1}$ & 0 & 0 & 0 & $J(\pi)$ & $n \mathcal{N}(\pi)^{2 / 3}$ & 0 \\
\hline$\theta_{6}^{1}$ & 0 & 0 & 0 & 0 & 0 & $n$ \\
\hline
\end{tabular}

and

\begin{tabular}{|c|c|c|c|c|c|c|}
\hline$\langle\cdot, \cdot\rangle$ & $\theta_{1}^{2}$ & $\theta_{2}^{2}$ & $\theta_{3}^{2}$ & $\theta_{4}^{2}$ & $\theta_{5}^{2}$ & $\theta_{6}^{2}$ \\
\hline$\theta_{1}^{2}$ & $n \mathcal{N}(\pi)^{2 / 3}$ & 0 & $-n$ & 0 & 0 & 0 \\
\hline$\theta_{2}^{2}$ & 0 & $n$ & 0 & 0 & 0 & 0 \\
\hline$\theta_{3}^{2}$ & $-n$ & 0 & $n \mathcal{N}(\pi)^{1 / 3}$ & 0 & 0 & 0 \\
\hline$\theta_{4}^{2}$ & 0 & 0 & 0 & $n \mathcal{N}(\pi)^{2 / 3}$ & 0 & $J(\pi)$ \\
\hline$\theta_{5}^{2}$ & 0 & 0 & 0 & 0 & $n$ & 0 \\
\hline$\theta_{6}^{2}$ & 0 & 0 & 0 & $\bar{J}(\pi)$ & 0 & $n \mathcal{N}(\pi)^{1 / 3}$ \\
\hline
\end{tabular}

From Proposition 3.6, one sees that $\theta^{0}$ belongs to the space spanned by $\theta_{1}^{1}$ and $\theta_{2}^{1}$. More precisely,

$$
\left\|\theta^{0}-\mathcal{N}(\pi)^{1 / 3} \theta_{1}^{1}-\mathcal{N}(\pi)^{-1 / 3} \theta_{2}^{1}\right\|=0
$$

We also remark that in both cases $h=2$ or $h=3, \theta^{h}$ belongs to the space spanned by $\theta_{1}^{h-1}, \theta_{2}^{h-1}$ and $\theta_{3}^{h-1}$. More precisely,

$$
\begin{array}{r}
\left\|\theta^{2}-\mathcal{N}(\pi)^{-1 / 3} \theta_{1}^{1}-\theta_{2}^{1}\right\|=0 \\
\left\|\theta^{3}-\mathcal{N}(\pi)^{-1 / 3} \theta_{1}^{2}-\mathcal{N}(\pi)^{1 / 3} \theta_{3}^{2}\right\|=0 .
\end{array}
$$

Moreover, from Proposition 3.6

$$
\left\langle\theta_{i}^{1}, \theta_{j}^{2}\right\rangle= \begin{cases}\mathcal{N}(\pi)^{-1 / 3} & \text { if }(i, j)=(2,1), \\ 0 & \text { otherwise. }\end{cases}
$$

Remark 3.2. Since $|J(\pi)|^{2}=n^{2} \mathcal{N}(\pi)$, by the Cauchy-Schwarz criterion, we deduce that $\theta_{4}^{1}$ and $\theta_{5}^{1}$ are proportional, as well as $\theta_{4}^{2}$ and $\theta_{6}^{2}$.

Actually, the remaining functions are independent, and after some linear combinations of them, one can show that

Proposition 3.7. The space $\Theta\left(\pi^{2}\right)$ is of dimension 5; an orthonomal basis of it is given by

$$
\begin{aligned}
\mathcal{B}(2)=\{ & \sqrt{\mathcal{N}(\pi)-1} \mathcal{N}(\pi)^{-1 / 6} \theta_{1}^{1}, \\
& \sqrt{\mathcal{N}(\pi)-1} \mathcal{N}(\pi)^{-1 / 6} \theta_{4}^{1}, \quad \sqrt{\mathcal{N}(\pi)-1} \theta_{3}^{1}, \\
& \left.\sqrt{\mathcal{N}(\pi)-1} \theta_{6}^{1}, \quad \mathcal{N}(\pi)^{-1 / 6} \theta_{1}^{1}+\mathcal{N}(\pi)^{1 / 6} \theta_{2}^{1}\right\}
\end{aligned}
$$


Proposition 3.8. The space $\Theta\left(\pi^{3}\right)$ is of dimension 10; an orthonomal basis of it is given by

$$
\begin{aligned}
\mathcal{B}(3)=\{ & \sqrt{\mathcal{N}(\pi)-1} \mathcal{N}(\pi)^{-1 / 6} \theta_{1}^{1}, & & \sqrt{\mathcal{N}(\pi)-1} \theta_{3}^{1}, \\
& \sqrt{\mathcal{N}(\pi)-1} \mathcal{N}(\pi)^{-1 / 6} \theta_{4}^{1}, & & \sqrt{\mathcal{N}(\pi)-1} \theta_{6}^{1}, \\
& \sqrt{\mathcal{N}(\pi)-1} \mathcal{N}(\pi)^{-1 / 3} \theta_{4}^{2}, & & \sqrt{\mathcal{N}(\pi)-1} \theta_{5}^{2}, \\
& \mathcal{N}(\pi)^{-1 / 6} \theta_{1}^{1}+\mathcal{N}(\pi)^{1 / 6} \theta_{2}^{1}, & & \sqrt{\mathcal{N}(\pi)-1} \theta_{2}^{2} \\
& \mathcal{N}(\pi)^{-1 / 3} \theta_{1}^{2}+\mathcal{N}(\pi)^{1 / 3} \theta_{3}^{2} & & \left.\mathcal{N}(\pi)^{-1 / 6} \theta_{1}^{1}+\mathcal{N}(\pi)^{1 / 6} \theta_{2}^{1}-\mathcal{N}(\pi)^{1 / 6} \theta_{1}^{2}\right\}
\end{aligned}
$$

The results of Section 3.2 .2 provide enough information to achieve an orthonormal basis of the space $\Theta\left(\pi^{h}\right)$ for any $h \geqslant 1$; this is the subject for a future work, as well as an interpretation, as it was shown in Chapter 1 , of this results as an explicit constant in the asymptotic behaviour of the cubic Kloosterman sums $K_{3}(m, n, c)$. 



\section{Cancellation of $S\left(a X^{3}+b X, c\right)$, for $c$ allmost prime}

In this chapter, we work with the cubic exponential sum

$$
S\left(a X^{3}+b X, c\right)=\sum_{x(c)} e\left(\frac{a x^{3}+b x}{c}\right),
$$

where $e(z)=\exp \left(2 i \pi\left(\operatorname{Tr}_{k / \mathbb{Q}}(z)\right)=\exp (2 i \pi(z+\bar{z}))\right.$, and $x$ runs through a representative set of $R / c R$. We consider this sum as a function of the parameter $c$, with $c$ belonging to the ring of integers $R$ of the field $k$.

We have already broached the horizontal Sato-Tate conjecture for the cubic exponential sums $S\left(a X^{3}+b X, c\right)$ in the Introduction. This conjecture has its origins in the parallel which can be made beetwen the cubic exponential sums $S\left(a X^{3}+b X, c\right)$ with $b \neq 0$ and the elliptic curves without complex multiplication, for which the Sato-Tate conjecture has been recently proved, under some mild additional condition. We find also this similarity beetwen cubic exponential sums with $b=0$ and elliptic curves with complex multiplication, where uniform distribution has been proved in both cases. For cubic exponential sums with $b=0$, it is a consequence of the resolution by Heath-Brown and Patterson of the Kummer conjecture about cubic Gauß sums (see [15]).

In our situation, i.e. when $b \neq 0$, the cubic Gauß sums are "replaced" by cubic Kloosterman sums, what is far from being insignificant, since we do not know how to make appear moments of order bigger than one for cubic Kloosterman sums, while this is achieved easily for cubic Gauss sums. In this sense, the cubic Kloosterman sums $K_{3}(m, n, c)$, or equivalently the cubic exponentila sums $S\left(a X^{3}+b X, c\right)$, are more difficult to handle. The belief in the horizontal Sato-Tate conjecture relies mostly on the vertical Sato-Tate conjecture, which holds, both for the classical Kloosterman sums $K(m, n, c)$ and for the cubic exponential sums (see Livné [30] and Katz [18]).

In all these aspects, we may consider $S\left(a X^{3}+b X, c\right)$ and $K(m, n, c)$ as being similar one to the other, and expect that the recent results of Fouvry and Michel (see [11]) about the distribution of the signs of Kloosterman sums over almost prime integers can be adapted to cubic exponential sums. This problem is within reach of what can be achieved by combining analytic number theoretic methods with the theory of automorphic forms and with algebraic geometric methods. More precisely, we shall present some sieve argument in Section 4.2, and apply it to the sums $S\left(a X^{3}+b X, c\right)$ in Section 4.3, using the essential fact, proved in Section 4.1, that these sums actually behave like a remainder term. This allows us to prove the following theorem in Section 4.3 .

Theorem 4.1. Let $g: \mathbb{R} \rightarrow \mathbb{R}$ be a smooth function with compact support in $[1,2]$ and let $X \geqslant 1$. Then, there exists a constant $C(g)$ depending only on $g$ and a non-negative function $h$ decreasing to 0 such that, for any $3 \leqslant u$, 


$$
\left|\sum_{\pi \mid c \Rightarrow \mathcal{N}(\pi) \geqslant X^{1 / u}} S\left(X^{3}+3 X, c\right) g\left(\frac{\mathcal{N}(c)}{X}\right)\right| \leqslant C(g) \frac{X}{\log X}\left(h(u)+\mathcal{O}\left(\frac{\log \log X}{\log X}\right)\right) .
$$

The second part of the work is made in Section 4.4, where we use the vertical Sato-Tate law to obtain a lower bound for the sums $S\left(a X^{3}+b X, c\right)$ :

Theorem 4.2. Let $g: \mathbb{R} \rightarrow \mathbb{R}$ be a smooth function with compact support in $[1,2]$ and let $X \geqslant 1$. Then, there exists a constant $C(g)$, such that, for any $3 \leqslant u$,

$$
\sum_{\pi \mid c \Rightarrow \mathcal{N}(\pi) \geqslant X^{1 / u}}\left|S\left(X^{3}+3 X, c\right)\right| g\left(\frac{\mathcal{N}(c)}{X}\right) \geqslant C^{\prime}(g) \frac{X}{\log X} .
$$

We remark here that it is only necessary to prove this for $u=3$; the other cases follow at once. Comparing Theorem 4.1 and Theorem 4.2 , we obtain the following result:

Theorem 4.3. Let $g: \mathbb{R} \rightarrow \mathbb{R}$ be a smooth function with compact support in $[1,2]$. Let $X \geqslant 1$. There exists an effectively computable constant $u$ such that

$$
\left|\sum_{\pi \mid c \Rightarrow \mathcal{N}(\pi) \geqslant X^{1 / u}} S\left(X^{3}+3 X, c\right) g\left(\frac{\mathcal{N}(c)}{X}\right)\right|<\sum_{\pi \mid c \Rightarrow \mathcal{N}(\pi) \geqslant X^{1 / u}}\left|S\left(X^{3}+3 X, c\right)\right| g\left(\frac{\mathcal{N}(c)}{X}\right) .
$$

Moreover, for such $u$,

$$
\begin{aligned}
& \sharp\left\{\mathcal{N}(c) \leqslant X: c \text { has less than } u \text { prime factors and } S\left(X^{3}+3 X, c\right)>0\right\} \gg \frac{X}{\log X}, \\
& \sharp\left\{\mathcal{N}(c) \leqslant X: c \text { has less than } u \text { prime factors and } S\left(X^{3}+3 X, c\right)<0\right\} \gg \frac{X}{\log X} .
\end{aligned}
$$

Theorem 4.3 is a step towards the expected formula (12) of the introductory chapter of this thesis. As we mentioned below formula (12) page 8, one should be able to give explicitely the contribution due to the "theta term", according to the calculations done in Chapter 3 , this shall be done in a future work.

The theory of automorphic forms that we need has been presented in Chapter 2 ; since this theory started with the fact that the Kubota symbol was a group morphism on some special subgroup of $\mathrm{SL}_{2}(\mathbb{Z}[\omega])$, all results so far were over the Eisenstein integers, which explains why we do not work over $\mathbb{Z}$. The particular case where $b=0$ is different in many ways of the case $b \neq 0$; namely, the cubic Gauß sums are defined by

$$
g_{3}(m, c)=\sum_{x(c)}\left(\frac{x}{c}\right)_{3} e\left(\frac{m x}{c}\right),
$$

and, for any rational prime $p \equiv 1(\bmod 3)$ splitting as $p=\pi, \bar{\pi}$ in $\mathbb{Z}[\omega]$, they satisfy

$$
2 \Re\left(g_{3}(a, \pi)\right)=\sum_{x=0}^{p-1} \exp \left(2 i \pi \frac{a x^{3}}{p}\right) .
$$


We do not have such a simple relation for the sums $S\left(a X^{3}+b X, c\right)$ with $b \neq 0$.

Finally, it should be pointed out that Theorem 4.2 does not gives a bound dependent on the parameter $u$, while Theorem 4.1 does. It is actually possible to improve Theorem 4.2 in this sense, by the use of algebraic geometric methods, and to obtain efficient bounds, leading to some explicit value of $u$. For example, in the case of the Kloosterman sums $K(m, n, c)$, the value $u=23$ has been obtained in [11], and was latter improved by Sivak in [43] to $u=22$, the improvement being realised in the sieve argument. Therefore, possibilities to improve the accuracy of Theorem 4.3 exist, either for the classical Kloosterman sums or for the cubic exponential sums; in the latter case, this is a work in progress.

\subsection{A non-trivial estimate in average}

In Chapter 2 and in Chapter 3 , we have been working with essential cusps; this was actually only necessarily in Chapter 3 , since Eisenstein series are defined only at essential cusps. In Chapter2 it was assumed for simplicity, since, as it was shown in Chapter[1. Poincaré series can be defined at any cusp.

Let $X$ be a positive real number. Let $g: \mathbb{R} \longrightarrow \mathbb{R}$ be a given function satisfying

$$
\left\{\begin{array}{l}
g \in C^{\infty} \\
g(t) \neq 0 \Longleftrightarrow t \in[1,2] .
\end{array}\right.
$$

Theorem 4.4. Let $m, n \in \mathbb{Z}[\omega]-\{0\}$. Then, for any $A>0$, there exist some $B>0$ such that

$$
\sum_{\substack{\mathcal{N}(d) \leqslant X^{1 / 2} \log -B \\ \operatorname{gcd}(d, 3)=1}} \sum_{\substack{c \equiv 0(d) \\ c \equiv 1(3)}} \frac{K_{3}(m, n, c)}{\mathcal{N}(c)^{1 / 2}} g\left(\frac{\mathcal{N}(c)}{X}\right) \ll X \log ^{-A} X,
$$

where the sum is taken over the primary integers $d$ of $\mathbb{Z}[\omega]$.

Proof. We shall use the results of Chapter2 There, for a primary integer $d$, we defined $\Gamma_{d}$ to be the group generated by $\Gamma_{0}^{*}(d)$ and $-I d$. Let $\sigma^{-1}=\left(\begin{array}{cc}d-1 & d-2 \\ d & d-1\end{array}\right)$ and $\tau^{-1}=I d$. We recall the convention that $\sigma=\tau$ if they are equivalent modulo $\Gamma_{d}$, so either $\sigma$ and $\tau$ have to be inequivalent for any $\Gamma_{d}$, or they have to be equal. Then for all $\gamma_{d}$, the cusps $\sigma^{-1}(\infty)$ and $\tau^{-1}(\infty)$ are two essential cusps, not $\Gamma_{d}$-equivalent. Such a choice of $\sigma$ and $\tau$ implies that $K l_{\sigma, \tau}(m, n, c)$ is well defined for any $\Gamma_{d}$.

Recall that, for $d \in \mathbb{Z}[\omega]$ and for a spectral parameter $(s, p) \in \mathbb{C} \times \mathbb{Z}$, we defined in Section 2.2.2 of Chapter 2 the quantities

$$
A_{m, n, \sigma, \tau}^{\mathrm{disc}}(d, s, p)=\sum_{u \in \mathcal{B}_{d}(s, p)} \overline{\rho_{u}}(\sigma, m) \rho_{u}(\tau, n),
$$

where $\mathcal{B}_{d}(s, p)$ is an orthonormal basis of the intersection of $\mathrm{L}^{2}\left(\Gamma_{d} \backslash G, \kappa\right)$ with the eigenspace of $\Delta$ of spectral parameter $(s, p)$; similarly, define then for $(s, p) \in i \mathbb{R} \times \mathbb{Z}$,

$$
A_{m, n, \sigma, \tau}^{\mathrm{cont}}(d, s, p)=\sum_{\sigma_{i} \in \mathcal{C}\left(\Gamma_{d}\right)} \bar{\psi}_{\sigma_{i}, \sigma}(s, m, p) \psi_{\sigma_{i}, \tau}(s, n, p) .
$$


Let $f: \mathbb{C} \longrightarrow \mathbb{R}$ be the smooth function defined by

$$
f(z)=g\left(\frac{\mathcal{N}(m n)^{1 / 2}}{\mathcal{N}(z) X}\right) \mathcal{N}(z)^{-1 / 2} \mathcal{N}(\sqrt{m n})^{1 / 2} .
$$

Then $f$ is a radial function with compact support in $\left[\mathcal{N}(m n)^{1 / 2} /(2 X), \mathcal{N}(m n)^{1 / 2} / X\right]$ and $\|f\|_{\infty} \ll_{m, n}$ $\|g\|_{\infty} X^{1 / 2}$. We start with Theorem 2.6 of Section 2.2.3 of Chapter2. It gives

$$
\begin{aligned}
\sum_{c} \frac{K_{\sigma, \tau}(c)}{\mathcal{N}(c)^{1 / 2}} & g\left(\frac{\mathcal{N}(c)}{X}\right)=\sum_{c} \frac{K_{\sigma, \tau}(c)}{\mathcal{N}(c)} f\left(\frac{\sqrt{m n}}{c}\right) \\
& =\sum_{(s, p)} \mathbf{K} f(s, p) A_{m, n}^{\text {disc }}(d, s, p)+\sum_{p \in \mathbb{Z}} \int_{(0)} \mathbf{K} f(s, p) A_{m, n}^{\text {cont }}(d, s, p) d s .
\end{aligned}
$$

From Cauchy-Schwarz we have, for * representing either the discrete case or the continuous case,

$$
\left|A_{m, n, \sigma, \tau}^{*}(d, s, p)\right| \leqslant\left(A_{m, m, \sigma, \sigma}^{*}(d, s, p)\right)^{1 / 2}\left(A_{n, n, \tau, \tau}^{*}(d, s, p)\right)^{1 / 2} .
$$

We shall have to separate the exceptional and non-exceptional spectrum. For the latter, we use (i) of Proposition 2.6 of Chapter 2 for $\mathbf{K} f(s, p)$, and we obtain that the non-exceptional part of the right hand side of 4.1 .2 is bounded by

$$
\begin{gathered}
\left|\sum_{\substack{(s, p) \\
s \in i \mathbb{R}}} \mathbf{K} f(s, p) A_{m, n}^{\mathrm{disc}}(d, s, p)+\sum_{p \in \mathbb{Z}} \int_{(0)} \mathbf{K} f(s, p) A_{m, n}^{\mathrm{cont}}(d, s, p) d s\right| \\
\leq\|f\|_{\infty} \sum_{\substack{(s, p) \\
s \in i \mathbb{R}}}(1+|s|)^{-a}(1+|p|)^{-b}\left(A_{m, m, \sigma, \sigma}^{\mathrm{disc}}(d, s, p)\right)^{1 / 2}\left(A_{n, n, \tau, \tau}^{\mathrm{disc}}(d, s, p)\right)^{1 / 2} \\
+\|f\|_{\infty} \sum_{p \in \mathbb{Z}} \int_{(0)}(1+|s|)^{-a}(1+|p|)^{-b}\left(A_{m, m, \sigma, \sigma}^{\mathrm{cont}}(d, s, p)\right)^{1 / 2}\left(A_{n, n, \tau, \tau}^{\mathrm{cont}}(d, s, p)\right)^{1 / 2} d s
\end{gathered}
$$

Again, by Cauchy-Schwarz, we obtain the upper bound

$$
\begin{aligned}
& X^{1 / 2}\left(\sum_{\substack{(s, p) \\
s \in i \mathbb{R}}} \frac{A_{m, m, \sigma, \sigma}^{\mathrm{disc}}(d, s, p)}{(1+|s|)^{a}(1+|p|)^{b}}\right)^{1 / 2}\left(\sum_{\substack{s, p) \\
s \in i \mathbb{R}}} \frac{A_{n, n, \tau, \tau}^{\mathrm{disc}}(d, s, p)}{(1+|s|)^{a}(1+|p|)^{b}}\right)^{1 / 2} \\
& +X^{1 / 2}\left(\sum_{p \in \mathbb{Z}} \int_{(0)} \frac{A_{m, m, \sigma, \sigma}^{\mathrm{cont}}(d, s, p)}{(1+|s|)^{a}(1+|p|)^{b}} d s\right)^{1 / 2}\left(\sum_{p \in \mathbb{Z}} \int_{(0)} \frac{A_{n, n, \tau, \tau}^{\mathrm{cont}}(d, s, p)}{(1+|s|)^{a}(1+|p|)^{b}} d s\right)^{1 / 2},
\end{aligned}
$$

what is bounded by 


$$
\begin{aligned}
& X^{1 / 2}\left(\sum_{\substack{(s, p) \\
s \in i \mathbb{R}}} \frac{A_{m, m, \sigma, \sigma}^{\mathrm{disc}}(d, s, p)}{(1+|s|)^{a}(1+|p|)^{b}}+\sum_{p \in \mathbb{Z}} \int_{(0)} \frac{A_{m, m, \sigma, \sigma}^{\mathrm{cont}}(d, s, p)}{(1+|s|)^{a}(1+|p|)^{b}} d s\right)^{1 / 2} \\
& \times\left(\sum_{\substack{(s, p) \\
s \in i \mathbb{R}}} \frac{A_{n, n, \tau, \tau}^{\mathrm{disc}}(d, s, p)}{(1+|s|)^{a}(1+|p|)^{b}}+\sum_{p \in \mathbb{Z}} \int_{(0)} \frac{A_{n, n, \tau, \tau}^{\mathrm{cont}}(d, s, p)}{(1+|s|)^{a}(1+|p|)^{b}} d s\right)^{1 / 2} .
\end{aligned}
$$

By the part (i) of Propostion 2.5 of Chapter 2, we conclude that the non-exceptional part is bounded by $O\left(X^{1 / 2}\right)$.

For the exceptional spectrum, we still can use the same estimate (i) of Proposition2.6 of Chap$\operatorname{ter} 2$ for $\mathbf{K} f(s, p)$ at $s=1 / 3$, but we have to use the weaker estimate (ii) of Proposition2.6 of Chapter 2 for $\mathbf{K} f(s, p)$ in the remaining exceptional spectrum; as we do not control the expression $A_{m, n, \sigma, \tau}^{\text {disc }}(d, 1 / 3,0)$ as the level $d$ varies, we simply remark that the former estimate $X^{-1 / 3}$ is bounded by the latter estimate $X^{1 / 3}$, and therefore we can keep the theta term with the rest of the exceptional spectrum. In the same way as above, we obtain

$$
\begin{gathered}
\left|\sum_{\substack{(s, p) \\
0<s \leqslant 1 / 3}} \mathbf{K} f(s, p) A_{m, n}^{\mathrm{disc}}(d, s, p)\right| \\
\leq\|f\|_{\infty} \sum_{\substack{(s, p) \\
0<s \leqslant 1 / 3}} X^{s_{i}}\left(A_{m, m, \sigma, \sigma}^{\mathrm{disc}}(d, s, p)\right)^{1 / 2}\left(A_{n, n, \tau, \tau}^{\mathrm{disc}}(d, s, p)\right)^{1 / 2} \\
\leq X^{1 / 2}\left(\sum_{\substack{s, p) \\
0<s \leqslant 1 / 3}} X^{s_{i}} A_{m, m, \sigma, \sigma}^{\mathrm{disc}}(d, s, p)\right)^{1 / 2}\left(\sum_{\substack{(s, p) \\
0<s \leqslant 1 / 3}} X^{s_{i}} A_{n, n, \tau, \tau}^{\mathrm{disc}}(d, s, p)\right)^{1 / 2} .
\end{gathered}
$$

Each of both sums can be estimated as

$$
\sum_{0<s_{i} \leqslant 1 / 3} X^{s_{i}} A_{*}^{\mathrm{disc}}\left(d, s_{i}, 0\right) \ll\left(\frac{X}{\mathcal{N}(d)^{2}}\right)^{s_{1}} \sum_{s_{i} \leqslant 1 / 3} \mathcal{N}(d)^{2 s_{i}} A_{*}^{\mathrm{disc}}\left(d, s_{i}, 0\right),
$$

whenever $\mathcal{N}(d)^{2} \leq X$. If such condition is fulfiled, the sum over the $i$ 's is controlled by part (ii) of Proposition 2.5 of Chapter2, giving

$$
\sum_{0<s_{i} \leqslant 1 / 3} X^{s_{i}} A_{*}^{\mathrm{disc}}\left(d, s_{i}, 0\right) \ll\left(\frac{X}{\mathcal{N}(d)^{2}}\right)^{s_{1}} \tau(d) \log ^{2} \mathcal{N}(d) \ll X^{s_{1}} \log ^{2} X \mathcal{N}(d)^{-2 s_{1}} \tau(d),
$$

the last estimation coming from the fact that we kept the theta term included in the whole exceptional spectrum. We conclude that for a given level $d$ and for $X$ big enough, i.e. $\mathcal{N}(d)^{2} \leq X$, we have

$$
\sum_{c} \frac{K_{\sigma, \tau}(c)}{\mathcal{N}(c)^{1 / 2}} g\left(\frac{\mathcal{N}(c)}{X}\right) \ll O\left(X^{1 / 2}+X^{1 / 2+s_{1}} \log ^{2} X \mathcal{N}(d)^{-2 s_{1}} \tau(d)\right) .
$$


With our choice of $\sigma$ and $\tau$, we apply Lemma 2.3. It remains to take the sum over the $d$ 's such that $\mathcal{N}(d) \leqslant Q$, for $Q=X^{1 / 2} \log ^{-B} X$. One obtains finally

$$
\sum_{\mathcal{N}(d) \leqslant Q} \sum_{c} \frac{K_{\sigma, \tau}(m, n, c)}{\mathcal{N}(c)^{1 / 2}} g\left(\frac{\mathcal{N}(c)}{X}\right) \ll X^{1 / 2+s_{1}} \log ^{2} X \sum_{\mathcal{N}(d) \leqslant Q} \frac{\tau(d)}{\mathcal{N}(d)^{2 s_{1}}} .
$$

At this point it is useful to remark that contrary to the non metaplectic case, where the maximal exceptional spectral parameter $s_{1}$ could be $1 / 2$, we do not here to improve on Selberg's estimate (compare with (2.3) p. 11 of [11]); actually the value $s_{1}=1 / 3$ corresponding through the Shimura correspondence to the value $s=2$ is already strictly smaller than $1 / 2$. It follows that the sum on the right hand side of 4.1 .3 is $\mathcal{O}\left(Q^{1-2 / 3}\right)$. Finally, with the value of $Q$ that we have chosen, it is $\mathcal{O}\left(X^{1 / 2-1 / 3} \log ^{-B(1-2 / 3)} X\right)$. We have therefore proved that

$$
\sum_{\mathcal{N}(d) \leqslant Q} \sum_{\substack{c \equiv 0(d) \\ c \equiv 1(3)}} \frac{K_{3}(m, n, c)}{\mathcal{N}(c)^{1 / 2}} g\left(\frac{\mathcal{N}(c)}{X}\right) \ll X \log ^{2-B / 3} X
$$

This finishes the proof of the theorem, taking $B \geqslant 3 A+6$.

Theorem 4.4 can be interpreted in terms of cubic exponential sums. Let $m, n \in \frac{1}{\lambda} \mathbb{Z}[\omega]$ and let $A, B, c$ be in $\mathbb{Z}[\omega]$. Then, if $\operatorname{gcd}(A, c)=\operatorname{gcd}(B, c)=1$ and if $27 m n A+B^{3}=0$, we have

$$
K_{3}(m, n, c)=S\left(A X^{3}+B X, c\right)\left(\frac{m n^{2}}{c}\right)_{3} .
$$

As $c$ varies, the condition that $B$ should be coprime to $c$ complicates the situation; therefore, we shall simply make the choice $m=n=1$. Our preceding result in this particular case gives:

Corollary 4.1. Let $g$ be a compactly supported smooth function with support in $[1,2]$. Let $X \geqslant 1$. Then, for any $A>0$, there exists $B>0$, such that

$$
\sum_{\mathcal{N}(d) \leqslant \frac{X^{1 / 2}}{\log ^{B} X}} \sum_{\substack{c \equiv 0(d) \\ c \equiv 1(3)}} \frac{S\left(X^{3}+3 X, c\right)}{\mathcal{N}(c)^{1 / 2}} g\left(\frac{\mathcal{N}(c)}{X}\right) \ll X \log ^{-A} X .
$$

\subsection{Sieve argument on $\mathbb{Z}[\omega]$}

In this section, we present a particular sieve argument, the so called crible étrange, developed by Fouvry and Michel in [11]. It will permit us latter to obtain un upper bound for the sum of cubic exponential sums having less than a given number of prime factors. Adopting the classical notations used in sieve theory, the variable $z$ will always be a positive real number. As we work with the Eisenstein's integers, we shall denote a prime integer by $\pi$, and any integer by $c$. The symbol $(a, b)$ will represent the greatest common divisor, and $[a, b]$ the least common multiple. First of all, we define

$$
P=P(z)=\prod_{\mathcal{N}(\pi)<z} \pi .
$$

Now we consider a totally multiplicative function $\rho$ on $\mathbb{Z}[\omega]$, satisfying 


$$
\left\{\begin{array}{l}
0 \leqslant \rho(\pi) \leqslant A<\mathcal{N}(\pi), \quad \text { for all } \pi, \text { for some absolute constant } A \\
\sum_{\mathcal{N}(\pi) \leqslant X} \frac{\rho(\pi) \log \mathcal{N}(\pi)}{\mathcal{N}(\pi)}=2 \log X+\mathcal{O}(1)
\end{array}\right.
$$

By partial summation, such a function $\rho$ satisfies

$$
\sum_{\mathcal{N}(\pi)<X} \frac{\rho(\pi)}{\mathcal{N}(\pi)}=2 \log \log X+\mathcal{O}(1) .
$$

Then, we consider a sequence of real positive integers $\mathcal{A}=\left(a_{c}\right)_{c \in \mathbb{Z}[\omega]}$. Let us define, for any $d \in \mathbb{Z}[\omega]$, the subsequence $\mathcal{A}_{d}$ of $A$ by

$$
\mathcal{A}_{d}=\left\{a_{c} \in \mathcal{A}: c \equiv 0 \quad(\bmod d)\right\} .
$$

Then $\mathcal{A}_{1}=\mathcal{A}$. The sums of the terms of each sequence $\mathcal{A}_{d}$ is given by

$$
\left|\mathcal{A}_{d}\right|=\sum_{a_{c} \in \mathcal{A}_{d}} a_{c}
$$

and the quantity we are interested in is

$$
S(\mathcal{A}, z)=\sum_{a_{c} \in \mathcal{A}-\cup_{\pi \mid P} \mathcal{A}_{\pi}} a_{c}=\sum_{(\pi \mid c) \Rightarrow(\mathcal{N}(\pi) \geqslant z)} a_{c} .
$$

The hypothesis that usually appears concerning the sequence $\mathcal{A}$ is that the order of each local sum $\mathcal{A}_{d}$ is determined by $\rho(d)$, i.e.

$$
\left|\mathcal{A}_{d}\right|=\frac{\rho(d)}{\mathcal{N}(d)} Y+r(d)
$$

for some $Y \in \mathbb{R}$ and some term $r(d)$ behaving like a rest. The situation in which we shall later need to apply a sieve argument will be more complicated, in the sense that we shall consider the hypothesis: there exist $Y, Z \in \mathbb{R}$ such that for any $d \in \mathbb{Z}[\omega]$ holds

$$
\left|\mathcal{A}_{d}\right|=\frac{\rho(d)}{\mathcal{N}(d)} Y-\frac{\rho(d)}{\mathcal{N}(d)} \log \mathcal{N}(d) Z+r(d) .
$$

Related to the function $\rho$ it is convenient to introduce the totally multiplicative function $\rho^{*}$ defined on the primes by

$$
\rho^{*}(\pi)=\mathcal{N}(\pi)-\rho(\pi)
$$

as well as the function

$$
g(d)= \begin{cases}\rho(d) \rho^{*}(d)^{-1} & \text { if } d \text { square free } \\ 0 & \text { otherwise. }\end{cases}
$$

Finally we introduce the sums

$$
G_{c}(X)=\sum_{\substack{(d, c)=1 \\ \mathcal{N}(d) \leqslant X}} g(d)
$$


and

$$
G_{c}(X, z)=\sum_{\substack{d \mid P(z),(d, c)=1 \\ \mathcal{N}(d) \leqslant X}} g(d) .
$$

We shall denote $G_{1}(X)$ and $G_{1}(X, z)$ by $G(X)$ and $G(X, z)$ respectively. Then

$$
G_{c}(X, z) \begin{cases}\leqslant G_{c}(X) & \text { if } z<X, \\ =G_{c}(X) & \text { if } z \geqslant X .\end{cases}
$$

The following estimates will be usefull in the sequel:

$$
\begin{aligned}
\sum_{\mathcal{N}(\pi) \leqslant X} g(\pi) \log \mathcal{N}(\pi) & =2 \log X+\mathcal{O}(1) . \\
G_{c}(X, z) & =\mathcal{O}\left(\log ^{2} z\right)
\end{aligned}
$$

The first estimate is proved using the decomposition $g(\pi)=\frac{\rho(\pi)}{\mathcal{N}(\pi)}+\frac{\rho(\pi)}{\mathcal{N}(\pi)} g(\pi)$, the upper bound $g(\pi) \ll \frac{1}{\mathcal{N}(\pi)}$ and 4.2 .1 . For the second estimate, one simply notices that

$$
\begin{aligned}
G_{c}(X, z) & \leqslant \sum_{\mathcal{N}(d)<z} g(d)=\exp \left(\sum_{\mathcal{N}(\pi)<z} \log \left(\left(1-\frac{\rho(\pi)}{\mathcal{N}(\pi)}\right)^{-1}\right)\right) \\
& =\exp \left(\sum_{\mathcal{N}(\pi)<z} \frac{\rho(\pi)}{\mathcal{N}(\pi)}+\mathcal{O}\left(\frac{1}{\mathcal{N}(\pi)^{2}}\right)\right)=\exp (2 \log \log z+\mathcal{O}(1)),
\end{aligned}
$$

and one uses 4.2.2.

\subsubsection{The Selberg sieve}

In this section, we develop the sieve of Fouvry and Michel over the ring of Eisenstein integers $\mathbb{Z}[\omega]$; we shall prove the following theorem:

Theorem 4.5. Let $D \geqslant 1$. Let $\mathcal{A}$ be a sequence of positive real numbers satisfying the condition (4.2.3 $p$.121] for a totally multiplicative function $\rho$ satisfying (4.2.1), and for some $Y \in \mathbb{R}$ and $Z \in \mathbb{R}$. Then,

$$
\begin{gathered}
S(\mathcal{A}, z) \leqslant Y G(D, z)^{-1}+Z G(D, z)^{-2} \sum_{\mathcal{N}(\pi)<z} \frac{\rho(\pi) \mathcal{N}(\pi)}{\rho^{*}(\pi)^{2}} \log \mathcal{N}(\pi) G_{\pi}\left(\frac{D}{\mathcal{N}(\pi)}, z\right) \\
+\sum_{\substack{d \mid P \\
\mathcal{N}(d) \leqslant D^{2}}} 3^{\Omega(d)}|r(d)|,
\end{gathered}
$$

where $r(d)$ is the quantity defined in $(H)$. 
Proof. Selberg's method is the simplest to implement, and gives very good upper bound. We introduce a sequence of real number $\left(\lambda_{d}\right)_{d \geqslant 1}$ normalised such that $\lambda_{1}=1$ and $\lambda_{d}=0$ whenever $d \nmid P(z)$ or $\mathcal{N}(d) \geqslant D$. The parameter $D$ is the support of $\lambda$; It will be chosen later on, sufficiently small, to restrict the number of terms in the last sum, so that it behaves like an error term.

From now on, we write $P$ for $P(z)$. We have to find an upper bound for

$$
S(\mathcal{A}, z)=\sum_{(c, P(z))=1} a_{c}=\sum a_{c} \begin{cases}0 & \text { if }(c, P(z)) \neq 1 \\ 1 & \text { if }(c, P(z))=1 .\end{cases}
$$

Selberg's observation is that

$$
\left(\sum_{d \mid(c, P)} \lambda_{d}\right)^{2} \begin{cases}\geqslant 0 & \text { if }(c, P) \neq 1 \\ =1 & \text { if }(c, P)=1\end{cases}
$$

and therefore, we get

$$
\begin{aligned}
S(\mathcal{A}, z) & \leqslant \sum a_{c}\left(\sum_{d \mid(c, P)} \lambda_{d}\right)^{2}=\sum_{c} a_{c} \sum_{d_{1} \mid(c, P)} \sum_{d_{2} \mid(c, P)} \lambda_{d_{1}} \lambda_{d_{2}} \\
& =\sum_{c} a_{c} \sum_{d_{1}, d_{2} \mid P} \lambda_{d_{1}} \lambda_{d_{2}} \sum_{\left[d_{1}, d_{2}\right] \mid c} 1=\sum_{d_{1}, d_{2}} \lambda_{d_{1}} \lambda_{d_{2}} \sum_{c} a_{c} \sum_{\left[d_{1}, d_{2}\right] \mid c} 1 \\
& =\sum_{d_{1}, d_{2} \mid P} \lambda_{d_{1}} \lambda_{d_{2}} \sum_{\left[d_{1}, d_{2}\right] \mid c} a_{c}=\sum_{d_{1}, d_{2}} \lambda_{d_{1}} \lambda_{d_{2}}\left|\mathcal{A}_{\left[d_{1}, d_{2}\right]}\right| .
\end{aligned}
$$

Using the sieve hypothesis $(H)$ for $\left|\mathcal{A}_{\left[d_{1}, d_{2}\right]}\right|$, we obtain

$$
S(\mathcal{A}, z) \leqslant \sum_{d_{1}, d_{2}} \lambda_{d_{1}} \lambda_{d_{2}}\left(\frac{\rho\left(\left[d_{1}, d_{2}\right]\right)}{\mathcal{N}\left(\left[d_{1}, d_{2}\right]\right)} Y-\frac{\rho\left(\left[d_{1}, d_{2}\right]\right)}{\mathcal{N}\left(\left[d_{1}, d_{2}\right]\right)} \log \mathcal{N}\left(\left[d_{1}, d_{2}\right]\right) Z+r\left(\left[d_{1}, d_{2}\right], X\right)\right)
$$

i.e.

$$
\begin{gathered}
S(\mathcal{A}, z)=Y \underbrace{\sum_{d_{1}, d_{2}} \frac{\rho\left(\left[d_{1}, d_{2}\right]\right)}{\mathcal{N}\left(\left[d_{1}, d_{2}\right]\right)} \lambda_{d_{1}} \lambda_{d_{2}}}_{=: Q_{1}(\lambda)}-Z \underbrace{\sum_{d_{1}, d_{2}} \frac{\rho\left(\left[d_{1}, d_{2}\right]\right)}{\mathcal{N}\left(\left[d_{1}, d_{2}\right]\right)} \log \mathcal{N}\left(\left[d_{1}, d_{2}\right]\right) \lambda_{d_{1}} \lambda_{d_{2}}}_{=: Q_{2}(\lambda)} \\
+\underbrace{\sum_{d_{1}, d_{2}} r\left(\left[d_{1}, d_{2}\right], X\right) \lambda_{d_{1}} \lambda_{d_{2}}}_{=: R(\lambda)} .
\end{gathered}
$$

The method used by Fouvry and Michel to minimise $S(\mathcal{A}, z)$ is firstly to minimise $Q_{1}(\lambda)$, and then to transfer the value of $\lambda$ in $Q_{2}(\lambda)$. The quantities that we shall meet are 


$$
\begin{aligned}
x(a) & =\sum_{d} \frac{\rho(d)}{\mathcal{N}(d)} \lambda_{a d}, \\
x^{*}(a) & =\sum_{d} \frac{\rho(d)}{\mathcal{N}(d)} \log \mathcal{N}(d) \lambda_{a d} .
\end{aligned}
$$

Reformulation of $Q_{1}(\lambda)$ and $Q_{2}(\lambda)$ :

Remark that if $a b \mid P$, then $(a, b)=1$, and by the multipicativity of $\rho$ we obtain

$$
\lambda_{a b} \neq 0 \Rightarrow \rho(a b)=\rho(a) \rho(b) .
$$

Then,

$$
\begin{aligned}
Q_{1}(\lambda) & =\sum \frac{\rho\left(\left[d_{1}, d_{2}\right]\right)}{\mathcal{N}\left(\left[d_{1}, d_{2}\right]\right)} \lambda_{d_{1}} \lambda_{d_{2}}=\sum_{\substack{d_{1}^{\prime}, d_{2}^{\prime} \\
\left(d_{1}^{\prime}, d_{2}^{\prime}\right)=1}} \sum_{a} \frac{\rho\left(a d_{1}^{\prime} d_{2}^{\prime}\right)}{\mathcal{N}\left(a d_{1}^{\prime} d_{2}^{\prime}\right)} \lambda_{a d_{1}^{\prime}} \lambda_{a d_{2}^{\prime}} \\
& =\sum_{\substack{d_{1}^{\prime}, d_{2}^{\prime} \\
\left(d_{1}^{\prime}, d_{2}^{\prime}\right)=1}} \sum_{a} \frac{\rho(a) \rho\left(d_{1}^{\prime}\right) \rho\left(d_{2}^{\prime}\right)}{\mathcal{N}\left(a d_{1}^{\prime} d_{2}^{\prime}\right)} \lambda_{a d_{1}^{\prime}} \lambda_{a d_{2}^{\prime}} .
\end{aligned}
$$

As usual, the condition of coprimality is replaced by the introduction of the Möbius function.

$$
\begin{aligned}
Q_{1}(\lambda) & =\sum_{a} \sum_{d_{1}^{\prime}, d_{2}^{\prime}} \sum_{b \mid\left(d_{1}^{\prime}, d_{2}^{\prime}\right)} \mu(b) \frac{\rho(a) \rho\left(d_{1}^{\prime}\right) \rho\left(d_{2}^{\prime}\right)}{\mathcal{N}\left(a d_{1}^{\prime} d_{2}^{\prime}\right)} \lambda_{a d_{1}^{\prime}} \lambda_{a d_{2}^{\prime}} \\
& =\sum_{a} \sum_{b} \sum_{d_{1}, d_{2}} \mu(b) \frac{\rho(a) \rho\left(b d_{1}\right) \rho\left(b d_{2}\right)}{\mathcal{N}\left(a b^{2} d_{1} d_{2}\right)} \lambda_{a b d_{1}} \lambda_{a b d_{2}} \\
& =\sum_{a} \sum_{b} \sum_{d_{1}, d_{2}} \mu(b) \frac{\rho(a) \rho(b)^{2} \rho\left(d_{1}\right) \rho\left(d_{2}\right)}{\mathcal{N}\left(a b^{2} d_{1} d_{2}\right)} \lambda_{a b d_{1}} \lambda_{a b d_{2}} \\
& =\sum_{a} \sum_{b} \mu(b) \frac{\rho(a) \rho(b)^{2}}{\mathcal{N}\left(a b^{2}\right)} \sum_{d_{1}} \frac{\rho\left(d_{1}\right)}{\mathcal{N}\left(d_{1}\right)} \lambda_{a b d_{1}} \sum_{d_{2}} \frac{\rho\left(d_{2}\right)}{\mathcal{N}\left(d_{2}\right)} \lambda_{a b d_{2}} \\
& =\sum_{a} \sum_{b} \mu(b) \frac{\rho(a) \rho(b)^{2}}{\mathcal{N}\left(a b^{2}\right)} x(a b)^{2} \\
& =\sum_{d} \frac{\rho(d)}{\mathcal{N}(d)} x(d)^{2} \sum_{b \mid d} \mu(b) \frac{\rho(b)}{\mathcal{N}(b)}, \quad \text { since }(a, b) \neq 1 \Rightarrow x(a b)=0 .
\end{aligned}
$$

This finishes the transformation of $Q_{1}(\lambda)$. The first lines adapt to $Q_{2}(\lambda)$, taking into account the apparition of $\log \mathcal{N}\left(a d_{1}^{\prime} d_{2}^{\prime}\right)=\log \mathcal{N}(a)+\log \mathcal{N}\left(d_{1}^{\prime}\right)+\log \mathcal{N}\left(d_{2}^{\prime}\right)$, and following the same steps as above, we obtain 


$$
\begin{aligned}
Q_{2}(\lambda) & =\sum_{a} \sum_{b} \mu(b) \frac{\rho(a) \rho(b)^{2}}{\mathcal{N}\left(a b^{2}\right)} \log \mathcal{N}(a) \sum_{d_{1}} \frac{\rho\left(d_{1}\right)}{\mathcal{N}\left(d_{1}\right)} \lambda_{a b d_{1}} \sum_{d_{2}} \frac{\rho\left(d_{2}\right)}{\mathcal{N}\left(d_{2}\right)} \lambda_{a b d_{2}} \\
& +\sum_{a} \sum_{b} \mu(b) \frac{\rho(a) \rho(b)^{2}}{\mathcal{N}\left(a b^{2}\right)} \sum_{d_{1}} \frac{\rho\left(d_{1}\right)}{\mathcal{N}\left(d_{1}\right)} \log \mathcal{N}\left(b d_{1}\right) \lambda_{a b d_{1}} \sum_{d_{2}} \frac{\rho\left(d_{2}\right)}{\mathcal{N}\left(d_{2}\right)} \lambda_{a b d_{2}} \\
& +\sum_{a} \sum_{b} \mu(b) \frac{\rho(a) \rho(b)^{2}}{\mathcal{N}\left(a b^{2}\right)} \sum_{d_{1}} \frac{\rho\left(d_{1}\right)}{\mathcal{N}\left(d_{1}\right)} \lambda_{a b d_{1}} \sum_{d_{2}} \frac{\rho\left(d_{2}\right)}{\mathcal{N}\left(d_{2}\right)} \log \mathcal{N}\left(b d_{2}\right) \lambda_{a b d_{2}}
\end{aligned}
$$

and decomposing the $\log \mathcal{N}\left(b d_{i}\right)$, we get

$$
\begin{gathered}
Q_{2}(\lambda)=\sum_{a} \sum_{b} \mu(b) \frac{\rho(a) \rho(b)^{2}}{\mathcal{N}\left(a b^{2}\right)} \log \mathcal{N}(a) x(a b)^{2}+2 \sum_{a} \sum_{b} \mu(b) \frac{\rho(a) \rho(b)^{2}}{\mathcal{N}\left(a b^{2}\right)} \log \mathcal{N}(b) x(a b)^{2} \\
+2 \sum_{a} \sum_{b} \mu(b) \frac{\rho(a) \rho(b)^{2}}{\mathcal{N}\left(a b^{2}\right)} x(a b) \sum_{d} \frac{\rho(d)}{\mathcal{N}(d)} \log \mathcal{N}(d) \lambda_{a b d} \\
=\sum_{d} \frac{\rho(d)}{\mathcal{N}(d)} x(d)^{2} \sum_{b \mid d} \mu(b) \frac{\rho(b)}{\mathcal{N}(b)} \log \mathcal{N}\left(\frac{d}{b}\right)+2 \sum_{d} \frac{\rho(d)}{\mathcal{N}(d)} x(d) x^{\prime}(d) \sum_{b \mid d} \mu(b) \frac{\rho(b)}{\mathcal{N}(b)} \\
+2 \sum_{d} \frac{\rho(d)}{\mathcal{N}(d)} x(d)^{2} \sum_{b \mid d} \mu(b) \frac{\rho(b)}{\mathcal{N}(b)} \log \mathcal{N}(b) \\
=\sum_{d} \frac{\rho(d)}{\mathcal{N}(d)} \log \mathcal{N}(d) x(d)^{2} \sum_{b \mid d} \mu(b) \frac{\rho(b)}{\mathcal{N}(b)}+2 \sum_{d} \frac{\rho(d)}{\mathcal{N}(d)} x(d) x^{\prime}(d) \sum_{b \mid d} \mu(b) \frac{\rho(b)}{\mathcal{N}(b)} \\
+\sum_{d} \frac{\rho(d)}{\mathcal{N}(d)} x(d)^{2} \sum_{b \mid d} \mu(b) \frac{\rho(b)}{\mathcal{N}(b)} \log \mathcal{N}(b) .
\end{gathered}
$$

Since all the $d$ 's are square-free, we have

$$
\sum_{b \mid d} \mu(b) \frac{\rho(b)}{\mathcal{N}(b)}=\prod_{\pi \mid d}\left(1-\frac{\rho(\pi)}{\mathcal{N}(\pi)}\right)=\prod_{\pi \mid d} \frac{\rho^{*}(\pi)}{\mathcal{N}(\pi)}=\frac{\rho^{*}(d)}{\mathcal{N}(d)} .
$$

In conclusion, we have shown that

and

$$
Q_{1}(\lambda)=\sum_{d} \frac{\rho(d) \rho^{*}(d)}{\mathcal{N}(d)^{2}} x(d)^{2}
$$

$$
\begin{array}{r}
Q_{2}(\lambda)=\sum_{d} \frac{\rho(d) \rho^{*}(d)}{\mathcal{N}(d)^{2}} \log \mathcal{N}(d) x(d)^{2}+2 \sum_{d} \frac{\rho(d) \rho^{*}(d)}{\mathcal{N}(d)^{2}} x(d) x^{\prime}(d) \\
+\sum_{d} \frac{\rho(d)}{\mathcal{N}(d)} x(d)^{2} \sum_{b \mid d} \mu(b) \frac{\rho(b)}{\mathcal{N}(b)} \log \mathcal{N}(b) .
\end{array}
$$


We remark that $Q_{1}(x(d))$ is a diagonal quadratic form in $x(d)$.

Optimisation of $\lambda$ with respect to $Q_{1}$ :

Recall that according to 4.2.9), in order that $x(d) \neq 0$, we need $\lambda_{d d^{\prime}} \neq 0$ and in particular we need $\mathcal{N}(d) \leqslant D$ and $d \mid P=P(z)$. Moreover, one can inverse the formula 4.2.9 giving $x(d)$ in terms of $\lambda_{d}$ :

$$
\begin{aligned}
\sum_{c} \mu(c) \frac{\rho(c)}{\mathcal{N}(c)} x(d c) & =\sum_{c} \mu(c) \frac{\rho(c)}{\mathcal{N}(c)} \sum_{d^{\prime}} \frac{\rho\left(d^{\prime}\right)}{\mathcal{N}\left(d^{\prime}\right)} \lambda_{d c d^{\prime}} \\
& =\sum_{h} \frac{\rho(h)}{\mathcal{N}(h)} \lambda_{d h} \sum_{c \mid h} \mu(c)=\rho(1) \lambda_{d}=\lambda_{d} .
\end{aligned}
$$

From this last expression, we shall make appear the term $Q_{1}(\lambda)$. Namely, we have shown that

$$
\lambda_{d}=\sum_{d^{\prime}} \mu\left(d^{\prime}\right) \frac{\rho\left(d^{\prime}\right)}{\mathcal{N}\left(d^{\prime}\right)} x\left(d d^{\prime}\right),
$$

and in particular, for $d=1$, we obtain

$$
1=\lambda_{1}=\sum_{d} \mu(d) \frac{\rho(d)}{\mathcal{N}(d)} x(d) .
$$

Then, by Cauchy-Schwarz, we finally obtain

$$
\begin{aligned}
1 & =\left(\sum_{d} \mu(d) \frac{\rho(d)}{\mathcal{N}(d)} x(d)\right)^{2} \\
& =\left(\sum_{d \mid P} \mu(d) \frac{\sqrt{\rho(d)}}{\sqrt{\rho^{*}(d)}} \frac{\sqrt{\rho(d) \rho^{*}(d)}}{\mathcal{N}(d)} x(d)\right)^{2} \leqslant Q_{1}(\lambda) \sum_{\substack{d \mid P \\
\mathcal{N}(d) \leqslant D}} \frac{\rho(d)}{\rho^{*}(d)} .
\end{aligned}
$$

Remark that both hypothesis on $\rho$, namely $\rho(d) \geqslant 0$ and $\rho^{*}(d)>0$ have been used. Now, denoting by $\lambda^{\text {opt }}$ the value of $\lambda$ minimising $Q_{1}$, we see that it is determined by

$$
1=Q_{1}\left(\lambda^{\mathrm{opt}}\right)\left(\sum_{d \mid P(z), \mathcal{N}(d) \leqslant D} \frac{\rho(d)}{\rho^{*}(d)}\right),
$$

what amounts to

$$
Q_{1}\left(\lambda^{\mathrm{opt}}\right)=\left(\sum_{d \mid P(z), \mathcal{N}(d) \leqslant D} \frac{\rho(d)}{\rho^{*}(d)}\right)^{-1}=G(D, z)^{-1},
$$

where $G(D, z)$ has been defined in (4.2.4) p.121. Still, we miss the explicit value of $\lambda^{\text {opt }}$, or equivalently of the corresponding $x(d)$. For it, it suffices to find $x(d)$ such that $Q_{1}(\lambda)=G(D, z)^{-1}$. Writing $x(d)=\frac{\mu(d) \mathcal{N}(d)}{\rho^{*}(d)}$ gives $x(d)^{2}=\frac{\mathcal{N}(d)^{2}}{\rho^{*}(d)^{2}}$ and $Q_{1}(\lambda)=\sum \frac{\rho(d)}{\rho^{*}(d)}=G(D, z)$. Thus $\lambda^{\text {opt }}$ corresponds to 


$$
x^{\mathrm{opt}}(d)= \begin{cases}\frac{\mu(d) \mathcal{N}(d)}{\rho^{*}(d)} G(D, z)^{-1} & \text { if } d \mid P=P(z), \mathcal{N}(d)<D, \\ 0 & \text { otherwise. }\end{cases}
$$

The inversion formula

$$
\lambda_{d}=\sum_{d^{\prime} \mid P, \mathcal{N}\left(d^{\prime}\right) \leqslant D} \mu\left(d^{\prime}\right) \frac{\rho\left(d^{\prime}\right)}{\mathcal{N}\left(d^{\prime}\right)} x\left(d d^{\prime}\right)
$$

gives finally as optimale value of $\lambda$

$$
\begin{aligned}
\lambda_{d}^{\mathrm{opt}} & =\sum_{d d^{\prime} \mid P, \mathcal{N}\left(d^{\prime}\right) \leqslant D / \mathcal{N}(d)} \mu\left(d^{\prime}\right) \frac{\rho\left(d^{\prime}\right)}{\mathcal{N}\left(d^{\prime}\right)} \frac{\mu\left(d d^{\prime}\right) \mathcal{N}\left(d d^{\prime}\right)}{\rho^{*}\left(d d^{\prime}\right)} G(D, z)^{-1} \\
& =G(D, z)^{-1} \frac{\mu(d) \mathcal{N}(d)}{\rho^{*}(d)} G_{d}\left(\frac{D}{\mathcal{N}(d)}, z\right) .
\end{aligned}
$$

Before going further, let us prove a usefull property of $\lambda^{\text {opt}}$ : Let $d \in \mathbb{Z}[\omega]$ be a square-free integer, $d \mid P=P(z),(d) \leqslant D$. Then,

$$
\begin{aligned}
G(D, z) & =\sum_{\substack{d^{\prime} \mid P \\
\mathcal{N}\left(d^{\prime}\right) \leqslant D}} g\left(d^{\prime}\right)=\sum_{l \mid d} \sum_{\begin{array}{c}
\left.d^{\prime}, d\right)=l \\
d^{\prime} \mid P \\
\mathcal{N}\left(d^{\prime}\right) \leqslant D
\end{array}} g\left(d^{\prime}\right) \\
& =\sum_{l \mid d} g(l) \sum_{\substack{\left(d^{\prime}, d\right)=1 \\
d^{\prime} \mid P \\
\mathcal{N}\left(d^{\prime}\right) \leqslant / D \mathcal{N}(l)}} g\left(d^{\prime}\right) \geqslant \sum_{l \mid d} g(l) G_{d}\left(\frac{D}{\mathcal{N}(d)}, z\right) .
\end{aligned}
$$

Since $\sum_{l \mid d} g(l)=\mathcal{N}(d) \rho^{*}(d)^{-1}$, we obtain from 4.2.15) that

$$
\left|\lambda_{d}^{\mathrm{opt}}\right| \leqslant 1
$$

Evaluation of $Q_{2}\left(\lambda^{\mathrm{opt}}\right)$ :

Recall that (see equation 4.2.12)

$$
\begin{array}{r}
Q_{2}(\lambda)=\overbrace{\sum_{d} \frac{\rho(d) \rho^{*}(d)}{\mathcal{N}(d)^{2}} \log \mathcal{N}(d) x(d)^{2}}^{Q_{2,1}}+2 \overbrace{\sum_{\sum_{d} \frac{\rho(d) \rho^{*}(d)}{\mathcal{N}(d)^{2}} x(d) x^{\prime}(d)}^{Q_{2,2}}}^{+\underbrace{\sum_{d} \frac{\rho(d)}{\mathcal{N}(d)} x(d)^{2} \sum_{b \mid d} \mu(b) \frac{\rho(b)}{\mathcal{N}(b)} \log \mathcal{N}(b)}_{Q_{2,3}} .}
\end{array}
$$

The expression (4.2.15) of $\lambda^{\text {opt }}$ is not easy to handle, but in view of (4.2.17), we shall only need the expressions $x(d)$ and $x^{*}(d)$ corresponding to $\lambda^{\text {opt }}$. Denote them by $x^{\text {opt }}(d)$ and $x^{* \text { opt }}(d)$. Then, $x^{\text {opt }}(d)$ is given in 4.2.14). For $x^{* \text { opt }}(d)$, we remark that $x(d)$ and $x^{*}(d)$ are related as follows: 


$$
\begin{aligned}
x^{\prime}(d) & =\sum_{d^{\prime}} \frac{\rho\left(d^{\prime}\right)}{\mathcal{N}\left(d^{\prime}\right)} \log \mathcal{N}\left(d^{\prime}\right) \lambda_{d d^{\prime}} \\
& =\sum_{d^{\prime}} \frac{\rho\left(d^{\prime}\right)}{\mathcal{N}\left(d^{\prime}\right)} \sum_{\pi \mid d^{\prime}} \log \mathcal{N}(\pi) \lambda_{d d^{\prime}}=\sum_{\pi} \sum_{d^{\prime \prime}} \frac{\rho\left(\pi d^{\prime \prime}\right)}{\mathcal{N}\left(\pi d^{\prime \prime}\right)} \log \mathcal{N}(\pi) \lambda_{d \pi d^{\prime \prime}} \\
& =\sum_{\pi} \frac{\rho(\pi)}{\mathcal{N}(\pi)} \log \mathcal{N}(\pi) \sum_{d^{\prime}} \frac{\rho\left(d^{\prime}\right)}{\mathcal{N}\left(d^{\prime}\right)} \lambda_{d \pi d^{\prime}}=\sum_{\pi} \frac{\rho(\pi)}{\mathcal{N}(\pi)} \log \mathcal{N}(\pi) x(\pi d) .
\end{aligned}
$$

There exist a simple relation between $Q_{2,1}\left(\lambda^{\mathrm{opt}}\right)$ and $Q_{2,2}\left(\lambda^{\mathrm{opt}}\right)$. First of all, writing $x^{\prime}(d)$ in terms on $x(d)$ as above, we have

$$
\begin{aligned}
Q_{2,2}(\lambda) & =\sum_{d} \frac{\rho(d) \rho^{*}(d)}{\mathcal{N}(d)^{2}} x(d) x^{\prime}(d) \\
& =\sum_{d} \frac{\rho(d) \rho^{*}(d)}{\mathcal{N}(d)^{2}} x(d) \sum_{\pi} \frac{\rho(\pi)}{\mathcal{N}(\pi)} \log \mathcal{N}(\pi) x(\pi d) \\
& =\sum_{\pi} \frac{\rho(\pi)}{\mathcal{N}(\pi)} \log \mathcal{N}(\pi) \sum_{d} \frac{\rho(d) \rho^{*}(d)}{\mathcal{N}(d)^{2}} x(d) x(\pi d) .
\end{aligned}
$$

Recall that $x(d)$ is non-zero for $d$ square free, and thus, $x(\pi d)$ is non-zero only if $(\pi, d)=1$. Using now the expression $x^{\text {opt }}(d)$, we obtain

$$
\begin{aligned}
x^{\mathrm{opt}}(\pi d) & =\frac{\mu(\pi d) \mathcal{N}(\pi d)}{\rho^{*}(\pi d)} G(D, z)^{-1} \\
& =-\frac{\mu(d) \mathcal{N}(\pi) \mathcal{N}(d)}{\rho^{*}(\pi) \rho^{*}(d)} G(D, z)^{-1}=-\frac{\mathcal{N}(\pi)}{\rho^{*}(\pi)} x^{\mathrm{opt}}(d) .
\end{aligned}
$$

Replacing $x^{\text {opt }}(d)$ in terms of $x^{\text {opt }}(\pi d)$, we obtain

$$
\begin{aligned}
Q_{2,2}\left(\lambda^{\mathrm{opt}}\right) & =-\sum_{\pi} \frac{\rho(\pi)}{\mathcal{N}(\pi)} \log \mathcal{N}(\pi) \sum_{d} \frac{\rho(d) \rho^{*}(d)}{\mathcal{N}(d)^{2}} x(\pi d)^{2} \frac{\rho^{*}(\pi)}{\mathcal{N}(\pi)} \\
& =-\sum_{d} \frac{\rho(\pi d) \rho^{*}(\pi d)}{\mathcal{N}(\pi d)^{2}} x(\pi d)^{2} \sum_{\pi} \log \mathcal{N}(\pi) \\
& =-\sum_{d} \frac{\rho(d) \rho^{*}(d)}{\mathcal{N}(d)^{2}} x(d)^{2} \sum_{\pi \mid d} \log \mathcal{N}(\pi) \\
& =-\sum_{d} \frac{\rho(d) \rho^{*}(d)}{\mathcal{N}(d)^{2}} x(d)^{2} \log \mathcal{N}(d)=-Q_{2,1}\left(\lambda^{\mathrm{opt}}\right)
\end{aligned}
$$

For $Q_{2,3}$, we start with the inner sum 


$$
\begin{aligned}
& \sum_{b \mid d} \mu(b) \frac{\rho(b)}{\mathcal{N}(b)} \log \mathcal{N}(b)=\sum_{b \mid d} \mu(b) \frac{\rho(b)}{\mathcal{N}(b)} \sum_{\pi \mid b} \log \mathcal{N}(\pi) \\
& =\sum_{\pi \mid d} \log \mathcal{N}(\pi) \sum_{\pi|b| d} \mu(b) \frac{\rho(b)}{\mathcal{N}(b)}=\sum_{\pi \mid d} \log \mathcal{N}(\pi) \sum_{d^{\prime} \mid \frac{d}{\pi}} \mu\left(\pi d^{\prime}\right) \frac{\rho\left(\pi d^{\prime}\right)}{\mathcal{N}\left(\pi d^{\prime}\right)} \\
& =-\sum_{\pi \mid d} \frac{\rho(\pi)}{\mathcal{N}(\pi)} \log \mathcal{N}(\pi) \sum_{d^{\prime} \mid \frac{d}{\pi}} \mu\left(d^{\prime}\right) \frac{\rho\left(d^{\prime}\right)}{\mathcal{N}\left(d^{\prime}\right)} .
\end{aligned}
$$

Replacing the inner sum by the product

$$
\prod_{\pi^{\prime} \mid \frac{d}{\pi}}\left(1-\frac{\rho\left(\pi^{\prime}\right)}{\mathcal{N}\left(\pi^{\prime}\right)}\right)=\left(1-\frac{\rho(\pi)}{\mathcal{N}(\pi)}\right)^{-1} \prod_{\pi^{\prime} \mid d}\left(1-\frac{\rho\left(\pi^{\prime}\right)}{\mathcal{N}\left(\pi^{\prime}\right)}\right)
$$

gives us

$$
\begin{aligned}
\sum_{b \mid d} \mu(b) \frac{\rho(b)}{\mathcal{N}(b)} \log \mathcal{N}(b) & =-\sum_{\pi \mid d} \frac{\rho(\pi)}{\mathcal{N}(\pi)} \log \mathcal{N}(\pi)\left(1-\frac{\rho(\pi)}{\mathcal{N}(\pi)}\right)^{-1} \prod_{\pi^{\prime} \mid d}\left(1-\frac{\rho\left(\pi^{\prime}\right)}{\mathcal{N}\left(\pi^{\prime}\right)}\right) \\
& =-\frac{\rho^{*}(d)}{\mathcal{N}(d)} \sum_{\pi \mid d} \frac{\rho(\pi)}{\rho^{*}(\pi)} \log \mathcal{N}(\pi) .
\end{aligned}
$$

Inserting this expression in $Q_{2,3}$ leads to

$$
\begin{aligned}
Q_{2,3}(\lambda) & =-\sum_{d} \frac{\rho(d)}{\mathcal{N}(d)} x(d)^{2} \frac{\rho^{*}(d)}{\mathcal{N}(d)} \sum_{\pi \mid d} \frac{\rho(\pi)}{\rho^{*}(\pi)} \log \mathcal{N}(\pi) \\
& =-\sum_{d} \frac{\rho(d)}{\mathcal{N}(d)} x(d)^{2} \frac{\rho^{*}(d)}{\mathcal{N}(d)} \sum_{\pi \mid d}\left(\frac{\rho(\pi)}{\rho^{*}(\pi)}+1\right) \log \mathcal{N}(\pi)-\log \mathcal{N}(\pi) \\
& =-\sum_{d} \frac{\rho(d) \rho^{*}(d)}{\mathcal{N}(d)^{2}} x(d)^{2} \sum_{\pi \mid d} \frac{\mathcal{N}(\pi)}{\rho^{*}(\pi)} \log \mathcal{N}(\pi)+Q_{2,1} .
\end{aligned}
$$

Putting together 4.2.17, 4.2.18) and 4.2.19) gives finally

$$
\begin{aligned}
Q_{2}\left(\lambda^{\mathrm{opt}}\right) & =-\sum_{d} \frac{\rho(d) \rho^{*}(d)}{\mathcal{N}(d)^{2}} x^{\mathrm{opt}}(d)^{2} \sum_{\pi \mid d} \frac{\mathcal{N}(\pi)}{\rho^{*}(\pi)} \log \mathcal{N}(\pi) \\
& =-\sum_{\mathcal{N}(\pi)<z} \frac{\mathcal{N}(\pi)}{\rho^{*}(\pi)} \log \mathcal{N}(\pi) \sum_{\substack{d, \pi \mid d \\
\mathcal{N}(d) \leqslant D}} \frac{\rho(d) \rho^{*}(d)}{\mathcal{N}(d)^{2}} x^{\mathrm{opt}}(d)^{2} \\
& =-\sum_{\mathcal{N}(\pi)<z} \frac{\rho(\pi)}{\mathcal{N}(\pi)} \log \mathcal{N}(\pi) \sum_{\mathcal{N}(d) \leqslant \frac{D}{\mathcal{N}(\pi)}} \frac{\rho(d) \rho^{*}(d)}{\mathcal{N}(d)^{2}} x^{\mathrm{opt}}(\pi d)^{2} .
\end{aligned}
$$


We know that $x^{\text {opt }}(\pi d)^{2}=\mathcal{N}(\pi d)^{2} \rho^{*}(\pi d)^{-2} G(D, z)^{-2}$, when $\pi d \mid P$, i.e. $(\pi, d)=1$, and therefore,

$$
\begin{aligned}
Q_{2}\left(\lambda^{\mathrm{opt}}\right) & =-G(D, z)^{-2} \sum_{\mathcal{N}(\pi)<z} \frac{\rho(\pi) \mathcal{N}(\pi)}{\rho^{*}(\pi)^{2}} \log \mathcal{N}(\pi) \sum_{\substack{\mathcal{N}(d) \leqslant \frac{D}{\mathcal{N}(\pi)} \\
(\pi, d)=1}} \frac{\rho(d)}{\rho^{*}(d)} \\
& =-G(D, z)^{-2} \sum_{\mathcal{N}(\pi)<z} \frac{\rho(\pi) \mathcal{N}(\pi)}{\rho^{*}(\pi)^{2}} \log \mathcal{N}(\pi) G_{\pi}\left(\frac{D}{\mathcal{N}(\pi)}, z\right) .
\end{aligned}
$$

End of the proof:

It remains to bring together the relations (4.2.8), 4.2.13) and (4.2.20) to get the estimation of the main term. To finish the proof of Theorem 4.5 , it remains to deal with the remainder term. Since $\left|\lambda_{d}\right| \leqslant 1$ (see 4.2 .16$)$ ), we have

$$
\left|R\left(\lambda^{\mathrm{opt}}\right)\right|=\left|\sum_{d_{1}, d_{2}} \lambda_{d_{1}} \lambda_{d_{2}} r\left(\left[d_{1}, d_{2}\right]\right)\right| \leqslant \sum_{d_{1}, d_{2}}\left|r\left(\left[d_{1}, d_{2}\right]\right)\right|=\sum_{\substack{d \mid P \\ \mathcal{N}(d) \leqslant D^{2}}} 3^{\Omega(d)}|r(d)| .
$$

\subsubsection{Precise estimates}

This section deals with the sum $G_{c}(X, z)$ appearing in Theorem 4.5. Associated to $X$ and $z$, one usually defines the parameter

$$
\tau=\frac{\log X}{\log z} .
$$

If necessary, we shall denote $\tau$ by $\tau_{X}$, when $X$ will be changing. For us, the case of interest is when $z=X^{1 / u}$, i.e. $\tau>1$ fixed.

When $\rho$ satisfies 4.2 .1 p. 121 , it is known that the sum $G(X, z)$ over $\mathbb{Z}$ is closely related to the function $\sigma_{2}$ defined by

$$
\begin{gathered}
\sigma_{2}(u)=\frac{e^{-2 \gamma}}{8} u^{2} \quad \text { if } 0 \leqslant u \leqslant 2, \\
\left(u^{-2} \sigma_{2}(u)\right)^{\prime}=-2 u^{-3} \sigma_{2}(u-2) \quad \text { if } u>2,
\end{gathered}
$$

with $\sigma_{2}$ required to be continuous at $u=2$. It is a non-negative increasing function with $\lim _{u \rightarrow \infty} \sigma_{2}(u)=$ 1. If we define $\overline{\sigma_{2}}$ by

$$
\overline{\sigma_{2}}(u)=\int_{o}^{u} \sigma_{2}(t) d t
$$

then, one proves that 


$$
\begin{aligned}
\overline{\sigma_{2}}(u) & =\frac{e^{-2 \gamma}}{24} u^{3}, \quad \text { if } 0 \leqslant u \leqslant 2, \\
\sigma_{2}(u) & =3 \frac{\overline{\sigma_{2}}(u)}{u}-2 \frac{\overline{\sigma_{2}}(u-2)}{u}, \quad u>2, \\
\frac{\overline{\sigma_{2}}\left(2 u_{2}\right)}{u_{2}^{3}} & =\frac{\overline{\sigma_{2}}\left(2 u_{1}\right)}{u_{1}^{3}}-2 \int_{u_{1}}^{u_{2}} \frac{\overline{\sigma_{2}}(2 t-2)}{t^{4}} d t, \quad \text { if } 1 \leqslant u_{1} \leqslant u_{2} .
\end{aligned}
$$

We show that the sum $G_{c}(X, z)$ over $\mathbb{Z}[\omega]$ can also be determined asymptotically by $\sigma_{2}$. It is convenient to introduce the analogue of $\overline{\sigma_{2}}$ for $G_{c}(X, z)$, namely we define

$$
T_{c}(X, z)=\int_{1}^{X} G_{c}(t, z) \frac{d t}{t}
$$

\section{Lemma 4.1.}

$$
\log X G_{c}(X, z)=3 T_{c}(X, z)-2 T_{c}\left(\frac{X}{z}, z\right)+O\left(\log ^{-3} X\right) .
$$

Proof. We start with the following simple relation: Let $\pi \mid P(z), \pi \backslash c$ fixed; then

$$
\begin{aligned}
G_{c}(X, z)=\sum_{\substack{\mathcal{N}(d)<X, d \mid P(z) \\
(d, c)=1}} g(d) & =\sum_{\substack{\mathcal{N}(d)<X, d \mid P(z) \\
(d, c \pi)=1}} g(d)+\sum_{\substack{\mathcal{N}\left(d^{\prime}\right)<\frac{X}{\mathcal{N}(\pi)} \\
\left(d^{\prime}, c \pi\right)=1}} g\left(\pi d^{\prime}\right) \\
& =G_{c \pi}(X, z)+g(\pi) G_{c \pi}\left(\frac{X}{\mathcal{N}(\pi)}, z\right)
\end{aligned}
$$

that we formulate as

$$
G_{c \pi}(X, z)=G_{c}(X, z)-g(\pi) G_{c \pi}\left(\frac{X}{\mathcal{N}(\pi)}, z\right) .
$$

We start with the following sum:

$$
\begin{aligned}
& \sum_{\substack{\mathcal{N}(d)<X, d \mid P(z) \\
(d, c)=1}} g(d) \log \mathcal{N}(d)=\sum_{\substack{\mathcal{N}(d)<X, d \mid P(z) \\
(d, c)=1}} g(d) \sum_{\pi \mid d} \log \mathcal{N}(\pi) \\
& =\sum_{\substack{\mathcal{N}(\pi)<z \\
\pi X_{c}}} \log \mathcal{N}(\pi) \sum_{\substack{\mathcal{N}(d)<X, d \mid P(z) \\
\text { ald } \\
(d, c)=1}} g(d)=\sum_{\substack{\mathcal{N}(\pi)<z \\
\pi X_{c}}} g(\pi) \log \mathcal{N}(\pi) \sum_{\substack{\mathcal{N}(d)<\frac{X}{\mathcal{N}(\pi)}, d \mid P(z) \\
(d, c \pi)=1}} g(d) \\
& =\sum_{\substack{\mathcal{N}(\pi)<z \\
\pi X_{c}}} g(\pi) \log \mathcal{N}(\pi) G_{c \pi}\left(\frac{X}{\mathcal{N}(\pi)}, z\right),
\end{aligned}
$$

and from 4.2.26 with $X / \mathcal{N}(\pi)$ instead of $X$, this is equal to 


$$
\begin{aligned}
& \sum_{\substack{\mathcal{N}(\pi)<z \\
\pi X_{c}}} g(\pi) \log \mathcal{N}(\pi) G_{c}\left(\frac{X}{\mathcal{N}(\pi)}, z\right)-\sum_{\substack{\mathcal{N}(\pi)<z \\
\pi \chi_{c}}} g(\pi)^{2} \log \mathcal{N}(\pi) G_{c \pi}\left(\frac{X}{\mathcal{N}(\pi)^{2}}, z\right) \\
& =\sum_{\substack{\mathcal{N}(\pi)<z \\
\pi \chi_{c}}} g(\pi) \log \mathcal{N}(\pi) \sum_{\substack{\mathcal{N}(d)<\frac{X}{\mathcal{N}(\pi)}, d \mid P(z) \\
(d, c)=1}} g(d)-\sum_{\substack{\mathcal{N}(\pi)<z \\
\pi \chi_{c}}} g(\pi)^{2} \log \mathcal{N}(\pi) \sum_{\substack{\mathcal{N}(d)<\frac{X}{\mathcal{N}(\pi)^{2}}, d \mid P(z) \\
(c \pi, d)=1}} g(d) \\
& =\sum_{\substack{\mathcal{N}(d)<X, d \mid P(z) \\
(d, c)=1}} g(d) \sum_{\substack{\mathcal{N}(\pi)<\min \left(z, \frac{X}{\mathcal{N}(d)}\right) \\
\pi \chi_{c}}} g(\pi) \log \mathcal{N}(\pi)- \\
& \sum_{\substack{\mathcal{N}(d)<X, d \mid P(z) \\
(d, c)=1}} g(d) \sum_{\substack{\mathcal{N}(\pi)<\min \left(z, \sqrt{\frac{X}{\mathcal{N}(d)}}\right) \\
(\pi, d)=1 \\
\pi \chi_{c}}} g(\pi)^{2} \log \mathcal{N}(\pi) .
\end{aligned}
$$

The first term of the right hand side of 4.2.27) is

$$
\sum_{\substack{\mathcal{N}(d)<X, d \mid P(z) \\ \mathcal{N}(d)<\frac{X}{Z} \\(d, c)=1}} g(d) \sum_{\substack{\mathcal{N}(\pi)<z \\ \pi \chi_{c}}} g(\pi) \log \mathcal{N}(\pi)+\sum_{\substack{\mathcal{N}(d)<X, d \mid P(z) \\ \mathcal{N}(d) \geq \geq \\(d, c)=1}} g(d) \sum_{\substack{\mathcal{N}(\pi)<\mathcal{X} \\ \mathcal{N}(d)}} g(\pi) \log \mathcal{N}(\pi) .
$$

Using 4.2.6, we reorganise it as

$$
2 \sum_{\substack{\mathcal{N}(d)<X, d \mid P(z) \\(d, c)=1}} g(d) \log \frac{X}{\mathcal{N}(d)}-2 \sum_{\substack{\mathcal{N}(d)<\frac{X}{z}, d \mid P(z) \\(d, c)=1}} g(d) \log \left(\frac{X}{z \mathcal{N}(d)}\right)+O\left(\sum_{\substack{\mathcal{N}(d)<X, d \mid P(z) \\(d, c)=1}} g(d)\right) .
$$

For the second term of the right hand side of 4.2.27, we remark that the hypothesis 4.2.1 implies that

$$
\sum g(\pi)^{2} \log \mathcal{N}(\pi) \ll \sum \frac{\log \mathcal{N}(\pi)}{\mathcal{N}(\pi)^{2}}<\infty
$$

In conclusion, 4.2.27) can be written as

$$
\begin{aligned}
& \sum_{\substack{\mathcal{N}(d)<X, d \mid P(z) \\
(d, c)=1}} g(d) \log \mathcal{N}(d)=2 \sum_{\substack{\mathcal{N}(d)<X, d \mid P(z) \\
(d, c)=1}} g(d) \log \frac{X}{\mathcal{N}(d)} \\
& -2 \sum_{\substack{\mathcal{N}(d)<\frac{X}{z}, d \mid P(z) \\
(d, c)=1}} g(d) \log \left(\frac{X}{z \mathcal{N}(d)}\right)+O\left(\sum_{\substack{\mathcal{N}(d)<X, d \mid P(z) \\
(d, c)=1}} g(d)\right) .
\end{aligned}
$$


The left hand side of 4.2 .28 is equal to

$$
\sum_{\substack{\mathcal{N}(d)<X, d \mid P(z) \\(d, c)=1}} g(d) \log X-\sum_{\substack{\mathcal{N}(d)<X, d \mid P(z) \\(d, c)=1}} g(d) \log \frac{X}{\mathcal{N}(d)} .
$$

Now by partial summation, we have

$$
\sum_{\substack{\mathcal{N}(d)<X, d \mid P(z) \\(d, c)=1}} g(d) \log \frac{X}{\mathcal{N}(d)}=\int_{1}^{X} G_{c}(t, z) \frac{d t}{t}=T_{c}(X, z) .
$$

We conclude the proof of the lemma using 4.2.7).

\section{Proposition 4.1.}

$$
T_{c}(X, z)=C \overline{\sigma_{2}}(2 \tau) \log ^{3} z+\mathcal{O}\left(\frac{\log ^{3} X}{\log z} \tau^{3}\right)
$$

with

$$
C=\frac{\alpha^{2}}{2} e^{2 \gamma} \prod_{\pi}\left(1-\frac{1}{\mathcal{N}(\pi)}\right)^{2} \prod_{\pi X_{c}}(1+g(\pi))
$$

Proof. (i) Dividing the equation of Lemma 4.1 by $X \log ^{4} X$ and integrating from $X_{1}$ to $X_{2}$ gives

$$
\int_{X_{1}}^{X_{2}} \frac{G_{c}(t, z) d t}{t \log ^{3} t}=3 \int_{X_{1}}^{X_{2}} \frac{T_{c}(t, z) d t}{t \log ^{4} t}-2 \int_{X_{1}}^{X_{2}} \frac{T_{c}(t / z, z) d t}{t \log ^{4} t}+O\left(\frac{1}{\log ^{3} X_{1}}\right) .
$$

As the derivative of $\frac{T_{c}(t, z)}{\log ^{3} t}$ is $\frac{G_{c}(t, z)}{t \log ^{3} t}-3 \frac{T_{c}(t, z)}{t \log ^{4} t}$, 4.2.29) becomes

$$
\frac{T_{c}\left(X_{2}, z\right)}{\log ^{3} X_{2}}=\frac{T_{c}\left(X_{1}, z\right)}{\log ^{3} X_{1}}-2 \int_{X_{1}}^{X_{2}} \frac{T_{c}(t / z, z) d t}{t \log ^{4} t}+O\left(\frac{1}{\log X_{1}}\right),
$$

which is the analogous of 4.2.22 for $\overline{\sigma_{2}}$. It remains to prove that the quantity $R_{c}(X, z)=$ $T_{c}(X, z)-C \overline{\sigma_{2}}(2 \tau) \log ^{3} z$ behaves like a rest. Using 4.2.25), $R_{c}(X, z)$ satisfies

$$
\frac{R_{c}\left(X_{2}, z\right)}{\log ^{3} X_{2}}=\frac{R_{c}\left(X_{1}, z\right)}{\log ^{3} X_{1}}-2 \int_{X_{1}}^{X_{2}} \frac{R_{c}(t / z, z) d t}{t \log ^{4} t}+O\left(\frac{1}{\log X_{1}}\right) .
$$

(ii) From Lemma 4.1 with $X=z$, one derives the asymptotic formula for $T_{c}(X)$ (as in [14] $\mathrm{p}$. 149-151). Some simple modifications have to be done, leading to

$$
T_{c}(X)=\frac{\alpha^{2}}{6} \prod_{\pi}\left(1-\frac{1}{\mathcal{N}(\pi)}\right)^{2} \prod_{\pi X_{c}}(1+g(\pi)) \log ^{3} X\left(1+\mathcal{O}\left(\frac{1}{\log X}\right)\right) .
$$

(iii) From (ii) we show that $R_{c}(X, z)=\mathcal{O}\left(\tau^{3} \frac{\log ^{3} X}{\log z}\right)$. Assume $X<z$; then, from (ii) and from 4.2.23, we have that

$$
R_{c}(X, z)=T_{c}(X, z)-C \overline{\sigma_{2}}(2 \tau) \log ^{3} X=\mathcal{O}\left(\log ^{2} X\right), \quad \text { for } X<z .
$$

Combining 4.2.31) for $X_{2}=X$ and $X_{1}=z$ with 4.2.32), one obtains the desired result for the range $z \leqslant X<z^{2}$, i.e. for $1 \leqslant \tau<2$. An iterative argument on the range of $X$ concludes the proof of the proposition. 
We now give a corollary giving the estimates we shall need later. Note that this results are usefull for $\tau$ bounded from above.

Corollary 4.2. Let $z \leqslant X$ and let $\tau=\frac{\log X}{\log z}$. Then, for any integer $c$,

$$
\begin{gathered}
G_{c}(X, z)=\alpha^{2} e^{2 \gamma} \prod_{\pi}\left(1-\frac{1}{\mathcal{N}(\pi)}\right)^{2} \prod_{\pi \chi_{c}}\left(1-\frac{\rho(\pi)}{\mathcal{N}(\pi)}\right)^{-1} \sigma_{2}(2 \tau) \log ^{2} z\left(1+\mathcal{O}\left(\frac{\tau^{5}}{\log z}\right)\right), \\
G_{c}(X, z)^{-1}=\alpha^{-2} e^{-2 \gamma} \prod_{\pi}\left(1-\frac{1}{\mathcal{N}(\pi)}\right)^{-2} \prod_{\pi X_{c}}\left(1-\frac{\rho(\pi)}{\mathcal{N}(\pi)}\right) \sigma_{2}(2 \tau)^{-1} \log ^{-2} z\left(1+\mathcal{O}\left(\frac{\tau^{5}}{\log z}\right)\right) .
\end{gathered}
$$

Proof. From Lemma4.1 and Proposition 4.1, we get

$$
\begin{aligned}
\log X G_{c}(X, z) & =3 C \overline{\sigma_{2}}(2 \tau) \log ^{3} z-2 C \overline{\sigma_{2}}(2(\tau-1)) \log ^{3} z \\
& +O\left(\tau^{6} \log ^{2} z\right)+O\left((\tau-1)^{6} \log ^{2} z\right)+O\left(\log ^{2} X\right) .
\end{aligned}
$$

Since $\tau>1$, dividing by $\log X=\tau \log z$, and using property (4.2.24) of the function $\overline{\sigma_{2}}$, one obtains

$$
\begin{aligned}
G_{c}(X, z) & =3 C \frac{\overline{\sigma_{2}}(2 \tau)}{\tau} \log ^{2} z-2 C \frac{\overline{\sigma_{2}}(2(\tau-1))}{\tau} \log ^{2} z+O\left(\tau^{5} \log z+\frac{(\tau-1)^{6}}{\tau} \log z+\tau \log z\right) \\
& =3 C \frac{\overline{\sigma_{2}}(2 \tau)}{\tau} \log ^{2} z-2 C \frac{\overline{\sigma_{2}}(2 \tau-2)}{\tau} \log ^{2} z+O\left(\tau^{5} \log z\right) \\
& =2 C \sigma_{2}(2 \tau) \log ^{2} z+O\left(\tau^{5} \log z\right)=2 C \log ^{2} z O\left(\sigma_{2}(2 \tau)+\frac{\tau^{5}}{C \log z}\right) .
\end{aligned}
$$

\subsection{An upper bound for $S\left(a X^{3}+b X, c\right)$}

Recall that the parameter $\alpha$ is

$$
\alpha=\operatorname{Res}_{s=1} \zeta_{\mathbb{Q}(\omega)}(s) .
$$

we shall use the notation

$$
\zeta_{*}(s)=(s-1)^{2} \zeta_{\mathbb{Q}(\omega)}^{2}(s) .
$$

Our result concerning the cubic exponential sums $S\left(a X^{3}+b X, c\right)$ is Theorem 4.1 stated in the introduction of this chapter:

Theorem 4.6. Let $g: \mathbb{R} \longrightarrow \mathbb{R}$ a smooth function with compact support $[1,2]$ and let $u_{1}>0$ be fixed. Then there exists $c(g)$ and a non-negative function $h$ with $\lim _{u \rightarrow+\infty} h(u)=0$ such that, for any $u \leqslant u_{1}$, 


$$
\left|\sum_{\substack{c \in \mathbb{Z}[\omega] \\ \pi \mid c \Rightarrow \mathcal{N}(\pi) \geqslant X^{1 / u}}} g\left(\frac{\mathcal{N}(c)}{X}\right) \frac{S(A, B, c)}{\mathcal{N}(c)^{1 / 2}}\right| \leqslant c(g) \frac{X}{\log X}\left(h(u)+\mathcal{O}_{g, u_{1}}\left(\frac{\log \log X}{\log X}\right)\right) .
$$

Assume that $A$ et $B$ are chosen such that $S(A, B, c) \in \mathbb{R}$. Recall that

$$
P=P(z)=\prod_{\mathcal{N}(\pi)<z} \pi
$$

A priori, one would consider the sequence

and the sub-sequences

$$
\mathcal{A}=\left(a_{c}\right)_{c \in \mathbb{Z}[\omega]}, \quad \text { where } a_{c}:=g\left(\frac{\mathcal{N}(c)}{X}\right) \frac{S(A, B, c)}{\mathcal{N}(c)^{1 / 2}},
$$

$$
\mathcal{A}_{d}=\left\{a_{c} \in \mathcal{A}: c \equiv 0 \quad(\bmod d)\right\}, \quad \text { defined for any } d \in \mathbb{Z}[\omega] .
$$

The quantities we are interested in are

$$
\left|\mathcal{A}_{d}\right|=\sum_{a_{c} \in \mathcal{A}_{d}} a_{c} \quad \text { and } \quad S(\mathcal{A}, z)=\sum_{a_{c} \in \mathcal{A}-\cup_{\pi \mid P} \mathcal{A}_{\pi}} a_{c}=\sum_{\pi \mid c \Rightarrow \mathcal{N}(\pi) \geqslant z} a_{c} .
$$

The problem is that the sign of the elments of the sequence $\mathcal{A}$ changes, and a sieve argument in this case requires to work with the sums of type II

$$
\sum_{c} a_{d_{1} c} a_{d_{2} c}
$$

but as it is not known how to make appear these sums, one has to modify them and work with positive sequences. For it we use the weil upper bound in the case $\operatorname{gcd}(A, B, c)=1$ :

$$
|S(A, B, c)| \leqslant 2^{\nu(c)} \mathcal{N}(c)^{1 / 2},
$$

where $\nu(c)$ is the number of prime factors of $c$ (see [31], prop. 5.1).

The idea is to transform the sequence $\mathcal{A}$ in two positive sequences. The following inequality holds,

$$
\pm \frac{S(A, B, c)}{\mathcal{N}(c)^{1 / 2}}+2^{\Omega(c)} \geqslant 0
$$

where $\Omega(c)$ is the number of prime divisors of $c$ counted with multiplicity. It is, contrary to the function $\nu, \Omega$, a totally multiplicative function. Following Fouvry and Michel ([11]), we reformulate Theorem 4.6 as

$$
\begin{aligned}
& \sum_{\substack{c \in \mathbb{Z}[\omega] \\
\pi \mid c \Rightarrow \mathcal{N}(\pi) \geqslant x^{1 / u}}} g\left(\frac{\mathcal{N}(c)}{X}\right)\left( \pm \frac{S(A, B, c)}{\mathcal{N}(c)^{1 / 2}}+2^{\Omega(c)}\right)-\sum_{\substack{c \in \mathbb{Z}[\omega] \\
\pi \mid c \Rightarrow \mathcal{N}(\pi) \geqslant x^{1 / u}}} g\left(\frac{\mathcal{N}(c)}{X}\right) 2^{\Omega(c)} \\
& \leqslant c(g) \frac{X}{\log X}\left(h(u)+\mathcal{O}\left(\frac{\log \log X}{\log X}\right)\right) .
\end{aligned}
$$


Define now

$$
b_{c}=\left\{\begin{array}{ll}
g\left(\frac{\mathcal{N}(c)}{X}\right) 2^{\Omega(c)} & \text { if } \lambda \nmid c \\
0 & \text { if } \lambda \mid c
\end{array} \quad \text { and } \quad a_{c}^{ \pm}= \begin{cases} \pm g\left(\frac{\mathcal{N}(c)}{X}\right) \frac{S(A, B, c)}{\mathcal{N}(c)^{1 / 2}}+b_{c} & \text { if } \lambda \backslash c \\
0 & \text { if } \lambda \mid c\end{cases}\right.
$$

and according to this define the sequences

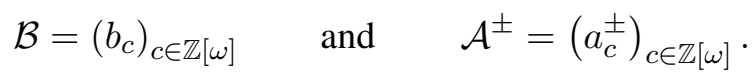

The quantities we are interested in are

$$
S\left(\mathcal{A}^{ \pm}, z\right)=\sum_{\substack{c \\ \pi \mid c \Rightarrow \mathcal{N}(\pi) \geqslant z}} a_{c}^{ \pm} \text {and } S(\mathcal{B}, z)=\sum_{\substack{c \\ \pi \mid c \Rightarrow \mathcal{N}(\pi) \geqslant z}} b_{c}
$$

Let $\hat{g}$ be the Mellin transform of $g$. Then, Theorem 4.6, and thereby Theorem4.1 of the introduction, is a consequence of the following:

Theorem 4.7. Let $3 \leqslant u$. We use the notations 4.3.5) and 4.3.6. There exists an absolute constant $C_{\mathrm{abs}}$ and a positive function $h$ decreasing to 0 , such that

$$
\left|S\left(\mathcal{A}^{ \pm}, X^{1 / u}\right)-S\left(B, X^{1 / u}\right)\right| \leqslant C_{\text {abs }} \hat{g}(1) \frac{X}{\log X}\left(h(u)+\mathcal{O}_{g, u}\left(\frac{1}{\log \log X}\right)\right) .
$$

Both sequences $\mathcal{A}^{ \pm}$and $\mathcal{B}$ are now positive (remark that they depend on $X$ ). Theorem 4.7 is proved if one can shows that

$$
\left|S\left(\mathcal{A}^{ \pm}, X^{1 / u}\right)\right| \leqslant C_{\text {abs }} \hat{g}(1) \frac{X}{\log X}\left(h_{1}(u)+\mathcal{O}_{g, u}\left(\frac{\log \log X}{\log X}\right)\right),
$$

and

$$
\left|S\left(B, X^{1 / u}\right)\right| \leqslant C_{\text {abs }} \hat{g}(1) \frac{X}{\log X}\left(h_{2}(u)+\mathcal{O}_{g, u}\left(\frac{\log \log X}{\log X}\right)\right),
$$

with $h_{1}(u), h_{2}(u) \rightarrow 0$ as $u \rightarrow+\infty$. The problem is that we shall show that

$$
S\left(\mathcal{B}, X^{1 / u}\right)=C_{\text {abs }} \hat{g}(1) \frac{X}{\log X} h_{3}(u)\left(+\mathcal{O}\left(\frac{1}{\log X}\right)\right),
$$

for some function $h_{3}(u)$ such that $h_{3}(u) \rightarrow+\infty$, as $u \rightarrow \infty$. Fortunately, one can be more precise, and prove that

$$
h_{3}(u)=\left(u^{2}+2 u\right)\left(1+h_{4}(u)\right), \quad u^{2} h_{4}(u) \rightarrow 0 .
$$

Therefore, to prove Theorem 4.7, one has to show that the contribution of $u$ in the main term of $S\left(\mathcal{B}, X^{1 / u}\right)$ is corrected by the one of $u$ in the main term of $S\left(\mathcal{A}^{ \pm}, X^{1 / u}\right)$, i.e one must prove that

$$
S\left(\mathcal{A}^{ \pm}, X^{1 / u}\right) \leqslant C_{\mathrm{abs}} \hat{g}(1) \frac{X}{\log X}\left(u^{2}+2 u\right)\left(1+h_{5}(u)\right)\left(+\mathcal{O}\left(\frac{\log \log X}{\log X}\right)\right),
$$


for some function $h_{5}(u)$ such that $u^{2} h_{5}(u) \rightarrow 0$, as $u \rightarrow \infty$. Then, substracting (4.3.8) from 4.3.7) proves Theorem 4.7, with $h(u)=\left(u^{2}+2 u\right) \max \left(h_{5}(u), h_{4}(u)\right)$.

In Section 4.3.1, we shall prove the upper bound 4.3.8), combining two topics: Firstly, the results issued from the theory of automorphic forms which were presented in Section 4.1, and secondly, the sieve argument of Section 4.2. In Section 4.3.2, the equality 4.3.7) is proved, by classical analytic methods.

\subsubsection{An upper bound for $\mathcal{A}^{ \pm}$}

In this section, we prove that the sequence $\mathcal{A}^{ \pm}$satisfies the conditions of Theorem 4.5 of Section 4.2.1, then apply Theorem 4.5 and use the estimates of Corollary 4.2 to obtain a first upper bound in the direction of 4.3.8). Then, Corollary 4.1 of Section 4.1 allows us to deal with the remainder term and to conclude the proof of 4.3.8.

Recall that

$$
S(\mathcal{B}, z)=\sum_{(c, P(z))=1} b_{c} \quad \text { and } \quad\left|\mathcal{B}_{d}\right|=\sum_{d \mid c} b_{c} .
$$

Since we avoid the prime $\lambda$, we shall work with primes of norm bigger than 4 . Denote by $\hat{g}$ the Mellin transform. It is defined by

$$
\hat{g}(s):=\int_{0}^{\infty} g(t) t^{s-1} d t \quad \text { and has inverse } g(t)=\frac{1}{2 i \pi} \int_{(\sigma)} g(s) t^{-s} d s .
$$

We need to introduce another notation. Let $c \in \mathbb{Z}[\omega]$; then we define the product

$$
F_{c}(s)=\prod_{\pi \chi_{c}}\left(1+\frac{1}{\mathcal{N}(\pi)^{s}\left(\mathcal{N}(\pi)^{s}-2\right)}\right)
$$

and write $F(s)$ for $F_{1}(s)$.

Lemma 4.2. Let $g$ be as given in Theorem 4.1 and let $\epsilon>0$. Then

$$
S(\mathcal{B}, 4)=X \log X \alpha^{2} \hat{g}(1) F(1)+X d(g)+O\left(X^{1-\epsilon}\right),
$$

with $d(g)$ a real constant depending only on the function $g$.

Proof. In the domain of absolute convergence, we have

$$
\begin{aligned}
\sum_{\lambda X_{c}} b_{c} & =\sum_{\lambda \chi_{c}} 2^{\Omega(c)} g\left(\frac{\mathcal{N}(c)}{X}\right)=\frac{1}{2 i \pi} \sum_{\lambda \chi_{c}} 2^{\Omega(c)} \int_{(\sigma)} \hat{g}(s)\left(\frac{\mathcal{N}(c)}{X}\right)^{-s} d s \\
& =\frac{1}{2 i \pi} \int_{(\sigma)} \hat{g}(s) \sum_{\lambda \chi_{c}} 2^{\Omega(c)}\left(\frac{\mathcal{N}(c)}{X}\right)^{-s} d s \\
& =\frac{1}{2 i \pi} \int_{(\sigma)} \hat{g}(s) \sum_{\lambda \chi_{c}} \frac{2^{\Omega(c)}}{\mathcal{N}(c)^{s}} X^{s} d s .
\end{aligned}
$$


Define

$$
L(s):=\sum_{\lambda \chi_{c}} \frac{2^{\Omega(c)}}{\mathcal{N}(c)^{s}}=\prod_{\pi \neq \lambda}\left(1-\frac{2}{\mathcal{N}(\pi)^{s}}\right)^{-1}
$$

and transform it as

$$
\begin{aligned}
L(s) & =\prod_{\pi \neq \lambda}\left(1-\frac{2}{\mathcal{N}(\pi)^{s}}\right)^{-1}=\prod_{\pi \neq \lambda} \frac{\mathcal{N}(\pi)^{s}}{\mathcal{N}(\pi)^{s}-2}=\prod_{\pi \neq \lambda} \frac{\mathcal{N}(\pi)^{2 s}}{\left(\mathcal{N}(\pi)^{s}-1\right)^{2}} \frac{\left(\mathcal{N}(\pi)^{s}-1\right)^{2}}{\mathcal{N}(\pi)^{s}\left(\mathcal{N}(\pi)^{s}-2\right)} \\
& =\prod_{\pi \neq \lambda}\left(1-\frac{1}{\mathcal{N}(\pi)^{s}}\right)^{-2} \prod_{\pi \neq \lambda}\left(1+\frac{1}{\mathcal{N}(\pi)^{s}\left(\mathcal{N}(\pi)^{s}-2\right)}\right)=\zeta_{\mathbb{Q}(\omega)}^{2}(s)\left(1-3^{-s}\right)^{2} F_{\lambda}(s) .
\end{aligned}
$$

Then,

$$
\begin{gathered}
\sum_{\lambda \chi_{c}} b_{c}=\frac{1}{2 i \pi} \int_{(\sigma)} \hat{g}(s) \zeta_{\mathbb{Q}(\omega)}^{2}(s)\left(1-3^{-s}\right)^{2} F_{\lambda}(s) X^{s} d s \\
=\frac{1}{2 i \pi} \int_{(1-\epsilon)} \hat{g}(s) \zeta_{\mathbb{Q}(\omega)}^{2}(s)\left(1-3^{-s}\right)^{2} F_{\lambda}(s) X^{s} d s+\operatorname{Res}_{s=1}\left(\hat{g}(s) \zeta_{\mathbb{Q}(\omega)}^{2}(s)\left(1-3^{-s}\right)^{2} F_{\lambda}(s) X^{s}\right) .
\end{gathered}
$$

We start with the residue, writing $\zeta_{*}(s)=\zeta_{\mathbb{Q}(\omega)}^{2}(s)(s-1)^{2}$,

$$
\begin{gathered}
\operatorname{Res}_{s=1}\left(\hat{g}(s) \zeta_{\mathbb{Q}(\omega)}(s)\left(1-3^{-s}\right)^{2} F_{\lambda}(s) X^{s}\right)=\lim _{s \rightarrow 1}\left(\left(\zeta_{*}(s) \hat{g}(s)\left(1-3^{-s}\right)^{2} F_{\lambda}(s) X^{s}\right)^{\prime}\right. \\
=X d(g)+X \log X \alpha^{2} \hat{g}(1) \frac{4}{9} F_{\lambda}(1)
\end{gathered}
$$

for some real constant $d(g)$ depending on the function $g$. In 4.3.11), the contribution in $X$ of the integral is less than the one of the residue, and therefore we obtain

$$
\sum_{\lambda X_{c}} b_{c}=X d(g)+X \log X \alpha^{2} \hat{g}(1) \frac{4}{9} F_{\lambda}(1)+\mathcal{O}\left(X^{1-\epsilon}\right),
$$

what proves the Lemma 4.2 .

With the notations of Lemma 4.2 , we define the real constants $Y=Y(X, g)$ and $Z=Z(X, g)$ by

$$
\left.\begin{array}{l}
Y=X \log X \alpha^{2} \frac{4}{9} \hat{g}(1) F_{\lambda}(1)+X d(g), \\
Z=X \alpha^{2} \frac{4}{9} \hat{g}(1) F_{\lambda}(1) .
\end{array}\right\}
$$


Corollary 4.3. Let $\epsilon>0$ and let $d \in \mathbb{Z}[\omega]$ such that $\lambda \nmid d$. Let $\mathcal{A}^{ \pm}$be the sequence defined in 4.3.5) and 4.3.6. Then, with $Y$ and $Z$ defined by 4.3.12), we have

$$
\left|\mathcal{A}_{d}^{ \pm}\right|=\frac{2^{\Omega(d)}}{\mathcal{N}(d)} Y-\frac{2^{\Omega(d)}}{\mathcal{N}(d)} \log \mathcal{N}(d) Z+r_{X}(d) .
$$

where $r_{X}(d)$ satisfies

$$
r_{X}(d)=\sum_{\substack{c \equiv 0(d) \\ \lambda X_{c}}} \frac{ \pm S\left(a X^{3}+b X, c\right)}{\mathcal{N}(c)^{1 / 2}} g\left(\frac{\mathcal{N}(c)}{X}\right)+O\left(2^{\Omega(d)}\left(\frac{X}{\mathcal{N}(d)}\right)^{1-\epsilon}\right) .
$$

Proof. Lemma 4.2, applied with $X / \mathcal{N}(d)$ instead of $X$, allows us to evaluate locally the sequence $\mathcal{B}$. We have

$$
\begin{aligned}
\left|\mathcal{B}_{d}\right| & =\sum_{d \mid c} b_{c}=\sum_{\lambda \chi_{c}} 2^{\Omega(d c)} g\left(\frac{\mathcal{N}(c)}{X / \mathcal{N}(d)}\right) \\
& =2^{\Omega(d)}\left\{\frac{X}{\mathcal{N}(d)} d(g)+\frac{X}{\mathcal{N}(d)} \log \left(\frac{X}{\mathcal{N}(d)}\right) \alpha^{2} \frac{4}{9} \hat{g}(1) F_{\lambda}(1)+O\left(\left(\frac{X}{\mathcal{N}(d)}\right)^{1-\epsilon}\right)\right\} \\
& =\frac{2^{\Omega(d)}}{\mathcal{N}(d)} X\left\{\log X \alpha^{2} \frac{4}{9} \hat{g}(1) F_{\lambda}(1)-\log \mathcal{N}(d) \alpha^{2} \frac{4}{9} \hat{g}(1)\right. \\
+\underbrace{\left(2_{\lambda}(1)+d(g)\right\}}_{=: r^{\prime}(d, X)} & +\underbrace{\left.\Omega(d)\left(\frac{X}{\mathcal{N}(d)}\right)^{1-\epsilon}\right)} .
\end{aligned}
$$

From this we deduce the local behaviour of the sequence $\mathcal{A}^{ \pm}$:

$$
\begin{aligned}
& \left|\mathcal{A}_{d}^{ \pm}\right|=\sum_{d \mid c} a_{c}^{ \pm}=\sum_{\substack{d \mid c \\
\lambda \chi_{c}}} \frac{ \pm S\left(a X^{3}+b, c\right)}{\mathcal{N}(c)^{1 / 2}} g\left(\frac{\mathcal{N}(c)}{X}\right)+\sum_{\substack{d \mid c \\
\lambda \chi_{c}}} b_{c} \\
& =\frac{2^{\Omega(d)}}{\mathcal{N}(d)} X\left\{\log X \alpha^{2} \frac{4}{9} \hat{g}(1) F_{\lambda}(1)-\log \mathcal{N}(d) \alpha^{2} \frac{4}{9} \hat{g}(1) F_{\lambda}(1)+d(g)\right\} \\
& +r^{\prime}(d, c)+\sum_{d \mid c} \frac{ \pm S\left(a X^{3}+b X, c\right)}{\mathcal{N}(c)^{1 / 2}} g\left(\frac{\mathcal{N}(c)}{X}\right) \text {. } \\
& \underbrace{\lambda X_{c}}_{r_{X}(d)}
\end{aligned}
$$

It is worthy of attention that the local behaviour of the sequence $\mathcal{A}^{ \pm}$expressed in Lemma 4.2 is an unsual sieve hypothesis. But, as it will become clear in this section, while the term $r_{X}(d)$ will be proven to be a real error term, the same will clearly not be the case for the "extra" term attached 
to $\log \mathcal{N}(d)$. This situation is the same as the one appearing in [11], for which Fouvry and Michel developed a new sieving process, namely the "crible étrange" presented in Section 4.2.1.

Before to apply Theorem 4.5 of Section 4.2.1, let us reformulate Corollary 4.2 of Section 4.2.2 in our situation. For it, we remark from Corollary 4.3 that our function $\rho$ is the one defined by

$$
\rho(\pi)= \begin{cases}2 & \text { if } \pi \neq \lambda, \\ 0 & \text { if } \pi=\lambda .\end{cases}
$$

Since we have (see [27] (67) p. 115)

$$
\sum_{\mathcal{N}(\pi) \leqslant X} \frac{\log \mathcal{N}(p)}{\mathcal{N}(p)}=\log X+O(1),
$$

the hypothesis 4.2.1 p. 121 of Section 4.2 is satisfied. Then, if $\tau=\frac{\log D}{\log z}$ and $(c, \lambda)=1$, Corollary 4.2 of Section 4.2 .2 gives

$$
\begin{aligned}
G_{c}(D, z) & =\alpha^{2} \frac{4}{9} e^{2 \gamma} F_{\lambda}(1) \prod_{\pi \mid c}\left(1-\frac{2}{\mathcal{N}(\pi)}\right) \sigma_{2}(2 \tau) \log ^{2} z\left(1+\mathcal{O}\left(\frac{\tau^{5}}{\log z}\right)\right), \\
G_{c}(D, z)^{-1} & =\alpha^{-2} \frac{9}{4} e^{-2 \gamma} F_{\lambda}(1)^{-1} \prod_{\pi \mid c} \frac{\mathcal{N}(\pi)}{\mathcal{N}(\pi)-2} \sigma_{2}(2 \tau)^{-1} \log ^{-2} z\left(1+\mathcal{O}\left(\frac{\tau^{5}}{\log z}\right)\right) .
\end{aligned}
$$

In particular, for a prime $\pi \neq \lambda$ such that $\mathcal{N}(\pi) \leqslant D$, we have $\tau-\frac{\log \mathcal{N}(\pi)}{\log z}=\mathcal{O}(\tau)$, and thus

$$
\begin{aligned}
G_{\pi}\left(\frac{D}{\mathcal{N}(\pi)}, z\right) & =\alpha^{2} \frac{4}{9} e^{2 \gamma} F_{\lambda}(1)\left(1-\frac{2}{\mathcal{N}(\pi)}\right) \\
& \times \sigma_{2}\left(2 \tau-2 \frac{\log \mathcal{N}(\pi)}{\log z}\right) \log ^{2} z\left(1+\mathcal{O}\left(\frac{\tau^{5}}{\log z}\right)\right) .
\end{aligned}
$$

We obtain now from Theorem 4.5 of Section 4.2.1, applied to the sequence $\mathcal{A}^{ \pm}$with $\rho$ given by 4.3.13, $Y$ and $Z$ given by 4.3.12 and $r_{X}(d)$ given by Corollary 4.3 , that

$$
\begin{gathered}
S\left(\mathcal{A}^{ \pm}, z\right) \leqslant Y G(D, z)^{-1}+2 Z G(D, z)^{-2} \sum_{\substack{\mathcal{N}(\pi)<z \\
\pi \neq \lambda}} \frac{\mathcal{N}(\pi)}{(\mathcal{N}(\pi)-2)^{2}} \log \mathcal{N}(\pi) G_{\pi}\left(\frac{D}{\mathcal{N}(\pi)}, z\right) \\
+\sum_{\substack{d \mid P(z) \\
\mathcal{N}(d) \leqslant D^{2}}} 3^{\Omega(d)}\left|r_{X}(d)\right|,
\end{gathered}
$$

for a parameter $D$ that still has to be chosen conveniently. As $z$ and $D$ will be chosen so that $z<D$, we can apply the estimate 4.3.16, and obtain 


$$
\begin{aligned}
& S\left(\mathcal{A}^{ \pm}, z\right) \leqslant Y G(D, z)^{-1}+ \\
& 2 \alpha^{2} \frac{4}{9} e^{2 \gamma} F_{\lambda}(1) Z G(D, z)^{-2} \log ^{2} z(1+\left.\mathcal{O}\left(\frac{\tau^{5}}{\log z}\right)\right) \sum_{\substack{\mathcal{N}(\pi)<z \\
\pi \neq \lambda}} \frac{\log \mathcal{N}(\pi)}{\mathcal{N}(\pi)-2} \sigma_{2}\left(2 \tau-2 \frac{\log \mathcal{N}(\pi)}{\log z}\right) \\
&+\sum_{\substack{d \mid P(z) \\
\mathcal{N}(d) \leqslant D^{2}}} 3^{\Omega(d)}\left|r_{X}(d)\right| \\
&=Y G(D, z)^{-1}+\frac{2 Z}{\sigma_{2}(2 \tau)} G(D, z)^{-1}\left(1+\mathcal{O}\left(\frac{\tau^{5}}{\log z}\right)\right) \sum_{\substack{\mathcal{N}(\pi)<z \\
\pi \neq \lambda}} \frac{\log \mathcal{N}(\pi)}{\mathcal{N}(\pi)-2} \sigma_{2}\left(2 \tau-2 \frac{\log \mathcal{N}(\pi)}{\log z}\right) \\
&+\sum_{\substack{d \mid P(z) \\
\mathcal{N}(d) \leqslant D^{2}}}^{\substack{\Omega(d) \\
3^{\Omega(d)}}}\left|r_{X}(d)\right|
\end{aligned}
$$

by 4.3.15), using the fact that $\frac{\tau^{5}}{\log z} \rightarrow 0$, as $z \rightarrow \infty$. Then, the property 4.2.22) p. 130 of $\sigma_{2}$ allows to make a partial integration and an easy calculation shows that

$$
\sum_{\substack{\mathcal{N}(\pi)<z \\ \pi \neq \lambda}} \frac{\log \mathcal{N}(\pi)}{\mathcal{N}(\pi)-2} \sigma_{2}\left(2 \tau-2 \frac{\log \mathcal{N}(\pi)}{\log z}\right)=\log z \sigma_{2}(2 \tau)+\mathcal{O}(1)
$$

Thus, as $\tau \geqslant 1$,

$$
\begin{aligned}
S\left(\mathcal{A}^{ \pm}, z\right) & \leqslant Y G(D, z)^{-1}+2 Z G(D, z)^{-1} \log z\left(1+\mathcal{O}\left(\frac{\tau^{5}}{\log z}\right)\right)+\sum_{\substack{d \mid P(z) \\
\mathcal{N}(d) \leqslant D^{2}}} 3^{\Omega(d)}\left|r_{X}(d)\right| \\
& =G(D, z)^{-1}(Y+2 Z \log z)+G(D, z)^{-1} \mathcal{O}\left(2 Z \tau^{5}\right)+\sum_{\substack{d \mid P(z) \\
\mathcal{N}(d) \leqslant D^{2}}} 3^{\Omega(d)}\left|r_{X}(d)\right|
\end{aligned}
$$

Now we see from 4.3.12) that

$$
Y+2 Z \log z=\alpha^{2} \frac{4}{9} \hat{g}(1) F_{\lambda}(1) X(\log X+2 \log z)\left(1+\mathcal{O}\left(\log ^{-1} z\right)\right)=\mathcal{O}(X \log X)
$$

and thus, from 4.3.15), we have

$$
G(D, z)^{-1}(Y+2 Z \log z)=e^{-2 \gamma} \frac{\hat{g}(1)}{\sigma_{2}(2 \tau)} X\left(\frac{\log X}{\log ^{2} z}+\frac{2}{\log z}\right)\left(1+\mathcal{O} \frac{\tau^{5}}{\log z}\right)=\mathcal{O}\left(\frac{X \log X}{\log ^{2} z}\right)
$$

This last estimate gives finally

$$
S\left(\mathcal{A}^{ \pm}, z\right) \leqslant e^{-2 \gamma} \frac{\hat{g}(1)}{\sigma_{2}(2 \tau)} X\left(\frac{\log X}{\log ^{2} z}+\frac{2}{\log z}\right)+\mathcal{O}\left(\frac{X \tau^{5} \log X}{\log ^{3} z}\right)+\sum_{\substack{d \mid P(z) \\ \mathcal{N}(d) \leqslant D^{2}}} 3^{\Omega(d)}\left|r_{X}(d)\right|
$$


Guided by (4.3.7), we have to make disappear the factor $\sigma_{2}(2 \tau)$. At this point, we now make the choice of $z$ and $D$. According to Corollary 4.1 of Section 4.1 we choose $D=X^{1 / 4} \log ^{-B} X$, for a free parameter $B$, and according to our goal, we choose $z=X^{1 / u}$, where $u$ is the variable we are most intersted in. We shall simply write $P$ for $P\left(X^{1 / u}\right)$. Then,

$$
\tau=\frac{u}{4}-B \frac{\log \log X}{\log X},
$$

and the Taylor expansion of $\sigma_{2}$ at $\frac{u}{2}$ reads

$$
\frac{1}{\sigma_{2}(2 \tau)}=\frac{1}{\sigma_{2}\left(\frac{u}{2}\right)}\left(1+\mathcal{O}\left(\frac{\log \log X}{\log X}\right)\right) .
$$

With this choice of $z$ and $D$, we have obtained

$$
S\left(\mathcal{A}^{ \pm}, X^{1 / u}\right) \leqslant \frac{e^{-2 \gamma} \hat{g}(1)}{\sigma_{2}(u / 2)} \frac{X}{\log X}\left(u^{2}+2 u\right)+\mathcal{O}_{B, u}\left(\frac{X \log \log X}{\log ^{2} X}\right)+\sum_{\substack{d \mid P \\ \mathcal{N}(d) \leqslant X^{1 / 2} \log ^{-B} X}} 3^{\Omega(d)}\left|r_{X}(d)\right| .
$$

Since

$$
\frac{1}{\sigma_{2}(1)}=1+\mathcal{O}\left(\left(\frac{t}{2}\right)^{-t / 2}\right)
$$

we have

$$
\begin{aligned}
S\left(\mathcal{A}^{ \pm}, X^{1 / u}\right) \leqslant e^{-2 \gamma} \hat{g}(1) & \frac{X}{\log X}\left(u^{2}+2 u\right)\left(1+\mathcal{O}\left(\left(\frac{t}{4}\right)^{-t / 4}\right)\right) \\
+ & \mathcal{O}_{B, u}\left(\frac{X \log \log X}{\log ^{2} X}\right)+\sum_{\substack{d \mid P \\
\mathcal{N}(d) \leqslant X^{1 / 2} \log ^{-B} X}} 3^{\Omega(d)}\left|r_{X}(d)\right|
\end{aligned}
$$

The last step to prove Inequality (4.3.8) is to show that $r_{X}(d)$ behaves like an error term. We shall not make use of the condition $d \mid P$, and we simply drop it. We start with Corollary 4.3 , which gives, for any $\varepsilon>0$, the following order of growth:

$$
\begin{aligned}
& \sum_{\substack{d \mid P \\
\mathcal{N}(d) \leqslant X^{1 / 2} \log -B X}} 3^{\Omega(d)}\left|r_{X}(d)\right|=\sum_{\substack{d \mid P \lambda \chi_{d} \\
\mathcal{N}(d) \leqslant X^{1 / 2} \log ^{-B} X}} 3^{\Omega(d)} \sum_{\substack{c \equiv 0(d) \\
\lambda \chi_{c}}} \frac{ \pm S\left(a X^{3}+b X, c\right)}{\mathcal{N}(c)^{1 / 2}} g\left(\frac{\mathcal{N}(c)}{X}\right) \\
& +\mathcal{O}\left(\sum_{\substack{\mathcal{N}(d) \leqslant P^{1 / 2} \chi_{d} \\
\mathcal{N} \log ^{-B} X}} 6^{\Omega(d)}\left(\frac{X}{\mathcal{N}(d)}\right)^{1-\varepsilon}\right) .
\end{aligned}
$$

Firstly, an application of Cauchy-Schwarz inequality gives 


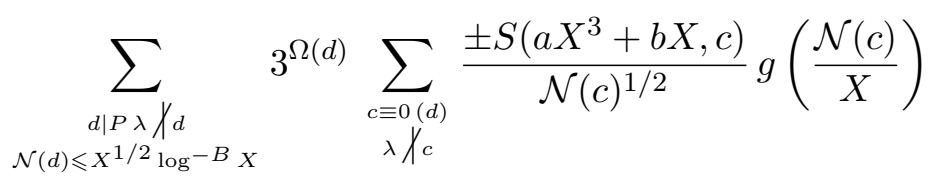

$$
\begin{aligned}
& \ll\left|\sum_{\substack{\lambda \chi_{d} \\
\mathcal{N}(d) \leqslant X^{1 / 2} \log ^{-B} X}} 9^{\Omega(d)} \sum_{\substack{c \equiv 0(d) \\
\lambda \chi_{c}}} \frac{ \pm S\left(a X^{3}+b X, c\right)}{\mathcal{N}(c)^{1 / 2}} g\left(\frac{\mathcal{N}(c)}{X}\right)\right|^{1 / 2} \\
& \cdot\left|\sum_{\substack{\lambda \chi_{d} \\
\mathcal{N}(d) \leqslant X^{1 / 2} \log ^{-B} X}} \sum_{\substack{c \equiv 0(d) \\
\lambda \chi_{c}}} \frac{ \pm S\left(a X^{3}+b X, c\right)}{\mathcal{N}(c)^{1 / 2}} g\left(\frac{\mathcal{N}(c)}{X}\right)\right|^{1 / 2} .
\end{aligned}
$$

Then, from the upper Weil bound applied to the terms $S\left(a X^{3}+b X, c\right)$ of the first factor we obtain

$$
\begin{aligned}
& \sum_{\substack{\lambda \chi_{d} \\
\mathcal{N}(d) \leqslant X^{1 / 2} \log ^{-B} X}} 3^{\Omega(d)} \sum_{\substack{c \equiv 0(d) \\
\lambda \chi_{c}}} \frac{ \pm S\left(a X^{3}+b X, c\right)}{\mathcal{N}(c)^{1 / 2}} g\left(\frac{\mathcal{N}(c)}{X}\right)
\end{aligned}
$$

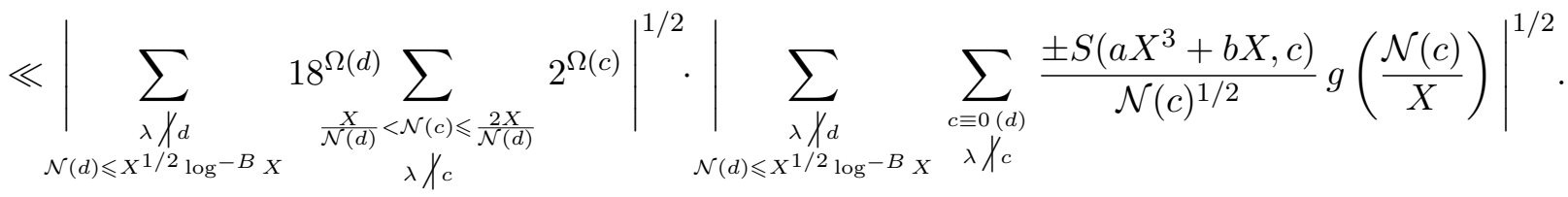

We recall the formula

$$
\sum_{\substack{\mathcal{N}(d) \leqslant X \\ \lambda X_{d}}} \frac{k^{\Omega(d)}}{\mathcal{N}(d)^{s}} \ll \begin{cases}X^{1-s} \log ^{k-1} X & \text { if } s \neq 1, \\ \log ^{k} X & \text { if } s=1 .\end{cases}
$$

From this formula applied in 4.3.19) we obtain

$$
\begin{aligned}
\sum_{\substack{d \mid P \\
\mathcal{N}(d) \leqslant X^{1 / 2} \log ^{-B} X}} 3^{\Omega(d)}\left|r_{X}(d)\right| \ll X^{1 / 2} \log ^{10} X \mid & \left.\sum_{\substack{\lambda \chi_{d} \\
\mathcal{N}(d) \leqslant X^{1 / 2} \log ^{-B} X}} \sum_{\substack{c \equiv 0(d) \\
\lambda X_{c}}} \frac{ \pm S\left(a X^{3}+b X, c\right)}{\mathcal{N}(c)^{1 / 2}} g\left(\frac{\mathcal{N}(c)}{X}\right)\right|^{1 / 2} \\
& +\mathcal{O}\left(X^{1-\varepsilon / 2} \log ^{5-B \varepsilon} X\right) .
\end{aligned}
$$

At this point we use the gain of the negative power of $\log X$ proved in the non trivial estimate in average of the cubic exponential sums. Corollary 4.1 of Section 4.1 applied with $A>24$ provides some $B>0$, such that

$$
\sum_{\substack{d \mid P \\ X^{1 / 2} \log -B}} 3^{\Omega(d)}\left|r_{X}(d)\right| \ll X \log ^{-2} X .
$$


This proves that the contribution of $r_{X}(d)$ is small enough to be considered as a rest, and (4.3.20) inserted in 4.3.17), gives

$S\left(\mathcal{A}^{ \pm}, X^{1 / u}\right) \leqslant e^{-2 \gamma} \hat{g}(1) \frac{X}{\log X}\left(u^{2}+2 u\right)\left(1+\mathcal{O}\left(\left(\frac{t}{4}\right)^{-t / 4}\right)\right)+\mathcal{O}_{B, u}\left(\frac{X \log \log X}{\log ^{2} X}\right)$,

and concludes the proof of 4.3.8.

\subsubsection{An upper bound for $\mathcal{B}$}

Here we prove 4.3.7). The sequence $\mathcal{B}=\left(b_{c}\right)$, wherev $b_{c}=2^{\Omega(c)} g\left(\frac{\mathcal{N}(c)}{X}\right)$ can be sieved exactly. For $z=X^{1 / u}$, the sum

$$
\sum_{\substack{\mathcal{N}(c) \leqslant X \\ \pi \mid c \Rightarrow \mathcal{N}(\pi) \geqslant z}} 2^{\Omega(c)}
$$

is close to the sum

$$
\sum_{\substack{\mathcal{N}(c) \leqslant X \\ \pi \mid c \Rightarrow \mathcal{N}(\pi) \geqslant z}} \tau(c)=\sum_{\substack{\mathcal{N}\left(c_{1}\right) \leqslant X \\ \pi \mid c_{1} \Rightarrow \mathcal{N}(\pi) \geqslant z}} \sum_{\substack{\mathcal{N}\left(c_{2}\right) \leqslant X \\ \pi \mid c_{2} \Rightarrow \mathcal{N}(\pi) \geqslant \frac{z}{\mathcal{N}\left(c_{1}\right.}}}
$$

The number of integers of norm less than $X$ having their prime factors of norm bigger than $z$ is known; for $z=Z^{1 / u}$, it is given by $u \omega(u) X \log ^{-1} X+\mathcal{O}\left(X \log ^{-2} X\right)$. One then shows that

$$
\sum_{\substack{\mathcal{N}(c) \leqslant X \\ \pi \mid c \Rightarrow \mathcal{N}(\pi) \geqslant z}} 2^{\Omega(c)}=u(2 \omega(u)+(\omega * \omega)(u)) \frac{X}{\log X}+\mathcal{O}\left(\frac{X}{\log ^{2} X}\right) .
$$

Moreover,

$$
u(2 \omega(u)+(\omega * \omega)(u))=e^{-2 \gamma}\left(u^{2}+2 u\right)\left(1+\mathcal{O}\left(\left(\frac{u}{4}\right)^{-u / 4}\right)\right)
$$

Therefore,

$$
\begin{aligned}
& S\left(\mathcal{B}, X^{1 / u}\right)=\sum_{\pi \mid c \Rightarrow \mathcal{N}(\pi) \geqslant X^{1 / u}} 2^{\Omega(c)} g\left(\frac{\mathcal{N}(c)}{X}\right)=-\sum_{\pi \mid c \Rightarrow \mathcal{N}(\pi) \geqslant X^{1 / u}} 2^{\Omega(c)} \int_{\mathcal{N}(c)}^{\infty} g^{\prime}\left(\frac{t}{X}\right) \frac{d t}{X} \\
& =-\int_{0}^{\infty} \sum_{\substack{\mathcal{N}(c) \leqslant t \\
\pi \mid c \Rightarrow \mathcal{N}(\pi) \geqslant X^{1 / u}}} 2^{\Omega(c)} g^{\prime}\left(\frac{t}{X}\right) \frac{d t}{X}
\end{aligned}
$$

and from above, one obtains

$$
\begin{aligned}
& S\left(\mathcal{B}, X^{1 / u}\right)=-\left(u^{2}+2 u\right)\left(1+\mathcal{O}\left(\left(\frac{u}{4}\right)^{-u / 4}\right)\right) \int_{0}^{\infty} \frac{X t}{\log X t}\left(1+\mathcal{O}\left(\frac{1}{\log X t}\right)\right) g^{\prime}(t) d t \\
& =-\left(u^{2}+2 u\right)\left(1+\mathcal{O}\left(\left(\frac{u}{4}\right)^{-u / 4}\right)\right) \frac{X}{\log X} \int_{0}^{\infty} \frac{t g^{\prime}(t)}{1+\log t / \log X}\left(1+\mathcal{O}\left(\frac{1}{\log (X t)}\right)\right) d t
\end{aligned}
$$


Since $g$ has compact support, the integral is

$$
\int_{0}^{\infty} t g^{\prime}(t) d t+\mathcal{O}\left(\frac{1}{\log X}\right)=-\hat{g}(1)+\mathcal{O}\left(\frac{1}{\log X}\right) .
$$

This proves 4.3.7), stated at the begin of Section 4.3, and thereby concludes the proof of Theorem4.7, as well as Theorem 4.1, stated at the begin of Chapter4

\subsection{A lower bound for $S\left(a X^{3}+b X, c\right)$}

In this section, we prove Theorem 4.2 stated at the begin of Chapter 4

\subsubsection{Sato-Tate vertical law}

If $a, b \in \mathbb{R}$, then

$$
\begin{aligned}
S\left(a X^{3}+b X, \pi\right) & =\sum_{x(\pi)} e\left(\frac{a x^{3}+b x}{\pi}\right)=\sum_{x=0}^{p-1} \exp \left(\frac{2 i \pi T r_{k / \mathbb{Q}}\left(\left(a x^{3}+b x\right) \bar{\pi}\right)}{p}\right) \\
& =\sum_{x=0}^{p-1} \exp \left(\frac{2 i \pi\left(a x^{3}+b x\right)(\pi+\bar{\pi})}{p}\right) \\
& =S_{\mathbb{Z}}\left(2 a \Re(\pi) X^{3}+2 b \Re(\pi) X, p\right),
\end{aligned}
$$

where $S_{\mathbb{Z}}(f(X), p)$ denotes the exponential sum

$$
S_{\mathbb{Z}}(f(X), p)=\sum_{x(p)} \exp \left(\frac{2 i \pi f(x)}{p}\right) .
$$

The integer $\pi+\bar{\pi}$ is coprime with $p$. In particular, as $a$ varies in $(\mathbb{Z} / p \mathbb{Z})^{\times}$, we have,

$$
\left\{S\left(a X^{3}+a X, \pi\right): a \in(R / \pi R)^{\times}\right\}=\left\{S_{\mathbb{Z}}\left(a X^{3}+a X, p\right): a=1, \ldots, p-1\right\} .
$$

One of the advantages of such equality is that $S_{\mathbb{Z}}(a, a, p)$ belongs to the class of exponential sums which satisfy the vertical Sato-Tate conjecture (see [10] p. 7). Let $\mu_{S T}$ be the sato-tate measure.

Proposition 4.2. For any arithmetical function $f$ such that $f(c)=0$ if $\mathcal{N}(c)>N$, and for $g$ as before, one has as $P \rightarrow \infty$,

$$
\begin{aligned}
\sum_{P \leqslant \mathcal{N}(\pi)<2 P} \sum_{\substack{c \\
\theta_{p, c} \in \mathcal{I}}} f(c) g\left(\frac{\mathcal{N}(\pi) \mathcal{N}(c)}{X}\right) & =\mu_{S T}(\mathcal{I}) \sum_{P \leqslant \mathcal{N}(\pi)<2 P} \sum_{c} f(c) g\left(\frac{\mathcal{N}(\pi) \mathcal{N}(c)}{X}\right) \\
& +\mathcal{O}\left(P(N / P+P)^{1 / 2}\left(\sum|f(c)|^{2}\right)^{1 / 2}\right) .
\end{aligned}
$$

This proposition is a consequence of the vertical Sato-Tate law; one proves it using the Large sievve inequality, exactly as in [10], Proposition 4.1. 


\subsubsection{Absolute lower bound}

For any $k \leqslant u$, from the twisted multiplicity, we can bound the sum from below by considering only $c$ being a square-free integer having exactly $k$ prime factors. Then,

$$
\begin{aligned}
& \sum_{\pi \mid c \Rightarrow \mathcal{N}(\pi) \geqslant X^{1 / u}}\left|S\left(X^{3}+3 X, c\right)\right| g\left(\frac{\mathcal{N}(c)}{X}\right) \\
& \geqslant \sum_{\mathcal{N}\left(\pi_{1}\right) \geqslant X^{1 / u}} \ldots \sum_{\mathcal{N}\left(\pi_{k}\right) \geqslant X^{1 / u}}\left|S\left(X^{3}+3 X, \pi_{1} \ldots \pi_{k}\right)\right| g\left(\frac{\mathcal{N}\left(\pi_{1} \ldots \pi_{k}\right)}{X}\right) \\
&=\sum_{\mathcal{N}\left(\pi_{1}\right) \geqslant X^{1 / u}} \ldots \sum_{\mathcal{N}\left(\pi_{k}\right) \geqslant X^{1 / u}}\left|S_{1}\left(X^{3}+3 X, \pi_{1}\right)\right| \ldots\left|S_{k}\left(X^{3}+3 X, \pi_{k}\right)\right|,
\end{aligned}
$$

where $S_{j}\left(X^{3}+3 X, \pi_{j}\right)=S\left(\left(\pi_{1} \ldots \pi_{j-1} \pi_{j+1} \ldots \pi_{k}\right)^{-1}\left(X^{3}+X\right), \pi_{j}\right)$, and $x^{-1}$ denotes an inverse of $x$ modulo the number given by the context.

In order to apply Proposition 4.2 , we need to restrict the range of each variable $\pi_{j}$. this can be realized for example by defining

and, for $j=2, \ldots k$, the sets

$$
\alpha_{j}=\frac{1}{u 2^{j}}+\frac{2^{j}-1}{32^{j}}
$$

$$
\mathcal{P}_{j}=\left\{X^{\alpha_{k-j}} 2^{n}: n \geqslant 0, X^{\alpha_{k-j}} 2^{n} \leqslant X^{\alpha_{k-j+1}} .\right.
$$

Then

$$
X^{\alpha_{j}} \leqslant P_{j} \leqslant X^{\alpha_{j+1}}, \quad \forall P_{j} \in \mathcal{P}_{j}
$$

Moreover, if we consider only the $S_{j}\left(X^{3}+3 X, \pi_{j}\right)$ whose angle $\theta_{j}$ belongs to $\mathcal{I}=[0, \xi] \cup[\pi-$ $\xi, \pi]$, then we have

$$
\frac{S_{j}\left(X^{3}+3 X, \pi_{j}\right)}{\mathcal{N}\left(\pi_{j}\right)^{1 / 2}}=2 \cos \theta_{j} \geqslant 2 \cos \xi, \quad \forall j .
$$

To simplify notations, write

$$
\mu\left(\xi_{j}\right)=\sum_{\pi_{1}} \sum_{P_{2} \leqslant \pi_{2}<2} \ldots \sum_{\substack{P_{2} \leqslant \pi_{k}<2 \\ \theta_{j} \in \mathcal{I}}} g\left(\frac{\mathcal{N}\left(\pi_{1} \ldots \pi_{k}\right)}{X}\right),
$$

and

$$
\mu(\xi)=\sum_{\pi_{1}} \sum_{P_{2} \leqslant \pi_{2}<2} \ldots \sum_{P_{2}} g\left(\frac{\mathcal{N}\left(\pi_{1} \ldots \pi_{k}\right)}{X}\right) .
$$

Then, from 4.4.1, we obtain

$$
\begin{aligned}
& \sum_{\pi \mid c \Rightarrow \mathcal{N}(\pi) \geqslant X^{1 / u}}\left|S\left(X^{3}+3 X, c\right)\right| g\left(\frac{\mathcal{N}(c)}{X}\right) \geqslant \sum_{P_{2} \in \mathcal{P}_{2}} \ldots \sum_{P_{k} \in \mathcal{P}_{k}} \mu\left(\xi_{1} \cap \ldots \cap \xi_{k}\right) \\
& \geqslant \sum_{P_{2} \in \mathcal{P}_{2}} \ldots \sum_{P_{k} \in \mathcal{P}_{k}}\left(\mu\left(\xi_{1}\right)+\ldots \mu\left(\xi_{k}\right)-(k-1) \mu(\xi)\right) .
\end{aligned}
$$


We shall estimate the quantity $\mu(\xi)$ by the prime number theorem, and the $\mu\left(\xi_{j}\right)$ by Proposition 4.2 :

$$
\mu\left(\xi_{j}\right)=\sum_{\pi_{1}} \sum_{P_{2} \leqslant \pi_{2}<2} \ldots \sum_{\substack{P_{2} \leqslant \pi_{k}<2 P_{k} \\ \theta_{j} \in \mathcal{I}}} g\left(\frac{\mathcal{N}\left(\pi_{1} \ldots \pi_{k}\right)}{X}\right)=\sum_{\pi_{1}} \sum_{\substack{c \\ \theta_{1}, c \in \mathcal{I}}} g\left(\frac{\mathcal{N}\left(\pi_{1} c\right)}{X}\right) f(c),
$$

where we define

$$
f(c)=\operatorname{Card}\left(\left\{\left(\pi_{2}, \ldots, \pi_{k}\right): c=\pi_{2} \ldots \pi_{k} \text { and } P_{i} \leqslant \pi_{i}<2 P_{i} \forall 2 \leqslant i \leqslant k\right\}\right) .
$$

When applying Proposition 4.2 , one sees that the rest term is bounded by $X \log ^{-2} X$ as soon as $k \geqslant 3$. Actually, considering large $k$ do not improve the result. Therefore, assuming $u \geqslant 3$, we fix $k=3$, and obtain from Proposition 4.2

$$
\mu\left(\xi_{j}\right)=\mu_{S T}(\mathcal{I}) \mu(\xi)+\mathcal{O}\left(\frac{X}{\log ^{2} X}\right) .
$$

Inserting 4.4.6) in 4.4.5) gives

$\sum_{\pi \mid c \Rightarrow \mathcal{N}(\pi) \geqslant X^{1 / u}}\left|S\left(X^{3}+3 X, c\right)\right| g\left(\frac{\mathcal{N}(c)}{X}\right) \geqslant \sum_{P_{2} \in \mathcal{P}_{2}} \sum_{P_{3} \in \mathcal{P}_{3}}\left(3 \mu_{S T}(\mathcal{I})-2\right) \mu(\xi)+\mathcal{O}\left(\frac{X}{\log ^{2} X}\right)$.

The prime number theorem and a partial summation give

$$
\begin{aligned}
\sum_{P_{2} \in \mathcal{P}_{2}} \sum_{P_{3} \in \mathcal{P}_{3}} \mu(\xi) & =\sum_{\pi_{1}} \sum_{X^{\alpha_{2}} \leqslant \pi_{2}<2} \sum_{X^{\alpha_{3}}} \sum_{X^{\alpha_{3}} \leqslant \pi_{3}<2 X^{\alpha_{4}}} g\left(\frac{\mathcal{N}\left(\pi_{1} \ldots \pi_{k}\right)}{X}\right) \\
& =\sum_{X^{\alpha_{2}} \leqslant \pi_{2}<2} \sum_{X^{\alpha_{3}}} \sum_{X^{\alpha_{3}} \leqslant \pi_{3}<2} \hat{g}(1) \frac{X}{\mathcal{N}\left(\pi_{2}\right) \mathcal{N}\left(\pi_{3}\right) \log X}\left(1+\mathcal{O}\left(\frac{\log X}{X}\right)\right) .
\end{aligned}
$$

Finally, the formula (see [27], (15) p. 150)

$$
\sum_{\mathcal{N}(\pi) \leqslant X} \frac{1}{\mathcal{N}(\pi)}=\log \log X+\mathcal{O}(1)
$$

shows that

$$
\sum_{\pi \mid c \Rightarrow \mathcal{N}(\pi) \geqslant X^{1 / u}}\left|S\left(X^{3}+3 X, c\right)\right| g\left(\frac{\mathcal{N}(c)}{X}\right) \gg\left(3 \mu_{S T}(\mathcal{I})-2\right) \hat{G}(1) \frac{X}{\log X} .
$$

By choosing $\xi$ such that $\mu_{S T}(\mathcal{I})=\frac{2}{\pi} \int_{0}^{\xi} \sin ^{2} \theta d \theta>\frac{2}{3}$, one concludes the proof of Theorem 4.2 p. 116: thereby we have proved our main theorem of this chapter, namely Theorem 4.3 p. 116. 



\section{Bibliography}

[1] B.J. Birch, How the number of points of an elliptic curve over a fixed prime field varies, J. London Math. Soc. 43 (1968), p. 57-60.

[2] C. Blondel, Les représentations supercuspidales des groupes métaplectiques sur GL(2) et leurs caractères, Mémoire de la S.M.F. 18 (1985), p. 1-119.

[3] C. Blondel, Uniqueness of Whittaker model for some supercuspidal representations of the metaplectic group, Comp. Math. 83 (1992), p. 1-18.

[4] R.W. Bruggeman, Y. Motohashi, Sum formula for Kloosterman sums and fourth moment of the Dedekind Zeta-function over the Gaussian number field, Funct. et Approx. 31 (2003), p. 23-92.

[5] W. Duke, J.B. Friedlander, H. Iwaniec, Equidistribution of Roots of a Quadratic Congruence to Prime moduli, Ann. of Math. 141 (1995), p. 423-441.

[6] F.G.M. Eisenstein, Beweis des Reciprocitätssatzes für die cubischen Reste in der Theorie der aus dritten Wurzeln der einheit zusammengesetzten complexen Zahlen, J. reine angew. Math. 27 (1844), p. 289-310; reprinted in Gotthold Eisenstein. Mathematische werke, Vol. 1, p. 59-80, New-York:Chelsea, 1975.

[7] F.G.M. Eisenstein, Nachtrag zum cubischen Reciprocitätsgesetze für die aus dritten Wurzeln der Einheit zusammengesetzten complexen Zahlen. Criterien des cubischen Characters de Zahl 3 und ihrer Teiler, J. reine angew. Math. 28 (1844), p. 28-35; reprinted in Gotthold Eisenstein. Mathematische werke, Vol. 1, p. 81-88, New-York:Chelsea, 1975.

[8] H.M. Farkas, I. Kra, Theta constant, Riemann surfaces and the modular group, Graduate Studies in Mathematics 37, A.M.S., Providence, RI, 2001.

[9] Y.Z. Flicker, Automorphic Forms on Covering Groups of GL(2), Invent. Math 57 (1980), p. 119-182.

[10] E. Fouvry, P. Michel, Sommes de modules de sommes d'exponentielles, Pacific J. Math. 209 (2003), p. 261-288.

[11] E. Fouvry, P. Michel, Sur le changement de signe des sommes de Kloosterman, Ann. of Math. 165 (2007), p. 675-715.

[12] D. Goldfeld, P. Sarnak, Sums of Kloosterman sums, Invent. Math. 71 (1983), p. 243-250.

[13] H. Lokvenec-Guleska, Sum formula for $\mathrm{SL}_{2}$ over imaginary quadratic number fields, $\mathrm{Ph} . \mathrm{D}$. Utrecht University (2004). 
[14] H. Halberstam, and H.E. Richert, Sieve Methods, London Math. Soc. Monographs 4, Academic Press, New-York, 1974.

[15] D.R. Heath-Brown, S.J. Patterson, The distribution of Kummer sums at prime argument, J. Reine Angew. Math. 310 (1979), p. 111-130.

[16] C. Hermite, Sur quelques formules relatives à la transformation des fonctions elliptiques, J. Math. pures et appliquées 3, ser. 2 (1858), p. 26-36; reprinted in Oeuvres de Charles Hermite, Tome 1, p. 487-496, Paris, Gauthier-Villars, 1905.

[17] C. Hooley, On the number of divisors of quadratic polynomials, Acta. Math. 110 (1963), p. 97-114.

[18] N.M. Katz, Sommes exponentielles, Astérisque 79 (1980).

[19] N.M. Katz, Gauss sums, Kloosterman Sums, and Monodromy groups, Ann. of Math. Stud. 116, Princeton Univ. Press, Princeton, NJ, 1988.

[20] D.A. Kazhdan, S.J. Patterson, Metaplectic forms, I.H.E.S. 59 (1984), p. 35-142.

[21] H.D. Kloosterman, On the representation of numbers in the form $a x^{2}+b y^{2}+c z^{2}+d t^{2}$, Acta Math. 49 (1926), p. 407-464.

[22] T. Kubota, Ein arithmetischer Satz über eine Matrizengruppe, J. Reine Angew. Math. 222 (1966), p. 55-57.

[23] T. Kubota, Topological covering of SL(2) over a local field, Journ. Math. Soc. Japan 19 (1967), p. 114-121.

[24] T. Kubota, On automorphic functions and the reciprocity law in a number field, Lectures in Mathematics, Department of Mathematics, Kyoto University (1969).

[25] T. Kubota, On a classical theta-function, Nagoya Math. J. 37 (1970), p. 183-189.

[26] N.V. Kuznetsov, Petersson's conjecture for cusp forms of weight zero and Linnik's conjecture, Sums of Kloosterman sums, Math. USSR Sbornik 39 (1981), p. 299-342.

[27] E. Landau, Über die zu einem algebraischen Zahlkörper gehörige Zetafunction und die Ausdehnung der Tschebyschefschen Primzahlentheorie auf das Problem der Verteilung der Primideale, J. Reine Angew. Math. 125 (1903), p. 64-188.

[28] Y. Linnik, Additive problems and eigenvalues of modular operators, Proc. I.C.M. Stockholm (1962), p. 270-284.

[29] R. Livné, The average distribution of cubic exponential sums, J. Reine Angew. Math. 375/376 (1987), p. 362-379.

[30] R. Livné, Cubic Exponential Sums and Galois Representations, Contemp. Math. 67 (1987), p. 247-261.

[31] R. Livné, S.J. Patterson, The first moment of cubic exponential sums, Invent. Math. 148 (2002) no. 1, p. 79-116. 
[32] S.J. Patterson, A cubic analogue of the theta series, J. Reine Angew. Math. 296 (1977), p. 125-161.

[33] S.J. Patterson, The level structure of exceptional metaplectic representations, J. Reine Angew. Math. 494 (1998), p. 85-100.

[34] S.J. Patterson, The cubic Shimura correspondence, Asian J. Math. 2 (1998) no.4, p. 957-982.

[35] H. Petersson, Zur analytischen Theorie der Grenzkreisgruppen, Math. Ann. 115 (1938), p. 23-67.

[36] N.V. Proskurin, On general Kloosterman sums (russian), Zap. Nauchn. Sem. S.-Peterburg Otdel. Mat. Inst. Steklov (POMI) 302 (2003), p. 107-134.

[37] W. Roelcke, Das Eigenwertproblem der automorphen Formen I,II, Math. Ann. 167 (1966), p. 292-337 + Math. Ann. 168 (1967), p. 261-324.

[38] H. Salié, Über die Kloostermanschen Summen S(u,v,q), Math. Z. 34 (1932), p. 91-109.

[39] P. Sarnak, Additive number theory and Maass forms, Number theory (New-York, 1982), Lecture Notes in Math. 1052, p. 286-309.

[40] A. Selberg, On the estimation of Fourier coefficients of modular forms, Proc. Symp. Pure Math. VIII A.M.S. (1965), p. 1-15.

[41] J.P. Serre, Abelian l-Adic Representations and Elliptic Curves, Second edition A K Peters, 1998.

[42] J.P. Serre, H.M. Stark, Modular forms of weight 1/2, Modular Functions of One Variable VI, Bonn (1976), p. 27-67.

[43] J. Sivak, Méthodes de crible appliquées aux sommes de Kloosterman et aux petits écarts entre nombres premiers, These, Université Paris-Sud, 2005.

[44] H. Weber, Elliptische Functionen und algebraische Zahlen, Braunschweig, 1891. 



\section{Acknowledgements}

I would like to thank my advisors Prof. Samuel Patterson and Prof. Philippe Michel, who gave me the possibility to discover the theory of exponential sums. During these years, I mostly worked with Prof. Samuel Patterson, whose deep insight in analytic number theory was a constant source of inspiration and motivation, and I had the opportunity to regularly benefit from the experience and valuable advices of Philippe Michel.

I would like to thank the Graduiertenkolleg Gruppen und Geometrie of Göttingen, which has partially supported this work and I would also like to thank the Mathematische Fakultät in Göttingen as well as the Université de Montpellier 2 and the EPFL of Lausanne for the opportunity to realize part of my work there, and for the financial support they provided for travel expenses.

Special thanks to Juan Marcos cerviño for its interest and for stimulating discussions, and in general to my collegues in all three institutions where I have found nice working atmosphere. 



\section{Abstract}

In this thesis work, we study the problem of the equidistribution of finite exponential sums. These sums possess an individual bound, and it is therefore natural to study the distribution of the normalized sums. More precisely, we deal with the case of Kloosterman sums twisted by the Legendre symbol of order 2 and 3 . The first case leads to the so-called Salie sums, and the second case leads to cubic exponential sums. We use the theory of metaplectic forms to study the behavior of these sums, and analyze in detail the contribution due to the cubic theta functions. We generalize the work of Livné and Patterson in two directions; the first problem is to obtain a trace formula and the second to work with metaplectic forms of any level. Then we develop a sieve method to study the problem of the equidistribution of cubic exponential sums over the almost prime Eisenstein integers. We are able to prove that the sign of these sums changes infinitely often, which is a result in direction of the Sato-Tate conjecture. 



\title{
Lebenslauf
}

\author{
Name: Benoît Louvel \\ Geburtsdatum: $\quad 16.02 .1980$ \\ Geburtsort: Rennes, France \\ Eltern: Paul Louvel, Yveline Louvel \\ Staatsangehörigkeit: Französisch
}

Schulbildung: September 1986 - Juni 1991

September 1991 - Juni 1995

September 1995 - Juni 1998

Studium: $\quad$ September 1998 - Juni 2003

$2001-2002$

April 2003 - August 2003
Grundschule am École publique Saint Germain sur Ille Weiter führende Schule am Collège Amand Brionne, Saint Aubin d'Aubigné Gymnasium am Lycée Ile de France Rennes

Mathematische Fakultät in der Universität Rennes Studiums-Auslandsjahr im Rahmen des internationalen Austauschprogramms ERASMUS, in Göttingen Studiums-Auslandssemester an der Universität Göttingen mit Abschlussarbeit: Groupes fuchsiens et approximations diophantiennes

Promotion an der Georg-August-Universität Göttingen und Université de Montpellier 2 